\title{
ANALYSIS OF WATER DIFFUSION IN WHITE MATTER USING A HYDRATION LAYER MODEL
}

\author{
by
}

\author{
Elena Olariu, M.Sc.
}

\author{
A thesis submitted to \\ the Faculty of Graduate Studies and Research \\ in partial fulfillment of \\ the requirements for the degree of \\ Doctor of Philosophy
}

Department of Physics

\section{Carleton University}

Ottawa, Ontario, Canada

September, 2009

@copyright

2009, Elena Olariu 
Library and Archives
Canada

Published Heritage

Branch

395 Wellington Street Ottawa ON K1A ON4 Canada
Bibliothèque et

Archives Canada

Direction du

Patrimoine de l'édition

395, rue Wellington

Ottawa ON K1A ON4

Canada
Your file Votre référence
ISBN: 978-0-494-60106-8
Our file Notre référence
ISBN: 978-0-494-60106-8
NOTICE:

The author has granted a nonexclusive license allowing Library and Archives Canada to reproduce, publish, archive, preserve, conserve, communicate to the public by telecommunication or on the Internet, loan, distribute and sell theses worldwide, for commercial or noncommercial purposes, in microform, paper, electronic and/or any other formats.

The author retains copyright ownership and moral rights in this thesis. Neither the thesis nor substantial extracts from it may be printed or otherwise reproduced without the author's permission.
AVIS:

L'auteur a accordé une licence non exclusive permettant à la Bibliothèque et Archives Canada de reproduire, publier, archiver, sauvegarder, conserver, transmettre au public par télécommunication ou par l'Internet, prêter, distribuer et vendre des thèses partout dans le monde, à des fins commerciales ou autres, sur support microforme, papier, électronique et/ou autres formats.

L'auteur conserve la propriété du droit d'auteur et des droits moraux qui protège cette thèse. $\mathrm{Ni}$ la thèse ni des extraits substantiels de celle-ci ne doivent être imprimés ou autrement reproduits sans son autorisation.
In compliance with the Canadian Privacy Act some supporting forms may have been removed from this thesis.

While these forms may be included in the document page count, their removal does not represent any loss of content from the thesis.
Conformément à la loi canadienne sur la protection de la vie privée, quelques formulaires secondaires ont été enlevés de cette thèse.

Bien que ces formulaires aient inclus dans la pagination, il n'y aura aucun contenu manquant.

\section{Canadä}




\section{Abstract}

Traditionally, diffusion in white matter has been interpreted in terms of restricted diffusion models with two well defined compartments: the intracellular space and the extracellular space. With these models the water molecules are normally considered to diffuse as if they were in pure water until they meet a barrier, such as the cell membrane, which simply reflects the particle back in a very classical sense. However, these models have not been successful in accounting for all of the observed results; in particular, the observed signal fractions are inconsistent with the predictions of these models.

The primary goal of this thesis was to propose a novel explanation for the observed diffusion properties of tissue water in human white matter in vivo by considering the electrostatic interactions of water molecules with each other and with their surroundings. These interactions lead to the formation of hydration layers around macromolecules and along membrane surfaces and cause the water in the cell to behave more like a gel than a liquid. Incorporation of these considerations into the analysis has allowed developing novel explanations for: 1) why the measured fast diffusion component is smaller than the diffusion 
coefficient of free water, 2) why it is anisotropic, 3) the observed signal fraction values and 4) the anisotropy of the signal fractions.

In this thesis, carefully measured sets of diffusion decays for the splenium and the genu of the corpus callosum of two healthy subjects in vivo are presented along with supporting $T_{2}$ relaxation time measurements. The diffusion decays were measured as a function of the angle between the diffusion direction and the long axis of the axon. All of these data are successfully interpreted in terms of the proposed hydration layer model. 


\section{Acknowledgements}

It is a pleasure to thank many people who made this thesis possible.

Foremost, I would like to thank my supervisor, Dr. Ian Cameron, who shared with me a lot of his expertise and research insight. Without his guidance, support, and encouragement this thesis would not have been possible. I also thank my committee members, Professors Boguslaw Jarosz and Richard Hodgson for their helpful suggestions and comments during the entire PhD program.

It is difficult to overstate my gratitude to Dr. Dave Rogers, Dr. Paul Johns, Dr. Peter Raaphorst, Dr. Malcolm McEwen and Dr. David Wilkins for teaching me the clinical aspects of medical physics. The road to my graduate degree has been long and winding, so I would also like to thank some people from the early days: Dr. Giles Santyr, Dr. Dean Karlen and Dr. Hans Mes.

I am indebted to my lab colleagues for providing a stimulating and fun environment in which to learn and grow: Claire Foottit, Marzieh Nezamzadeh, Arturo Cardenas-Blanco and Greg Cron. Huge thanks to Sorina Truica and Mihai Gherase for their valuable friendship. 
Thanks to many other people for Carleton University and the Ottawa General Hospital who have helped me in some manner during my studies.

Thanks to my beloved daughter Karina. Her love always gives me power!

Lastly, and most importantly, I wish to thank my parents. They have always supported and encouraged me to do my best in all matters of life. To them I dedicate this thesis.

And thanks to you all, who read and criticize my thesis! 


\section{Contents}

1 Introduction .......................................................................................................................

1.1 Overview of the MR Diffusion Literature ………...........................................

1.1.1 The Apparent Diffusion Coefficient .......................................................

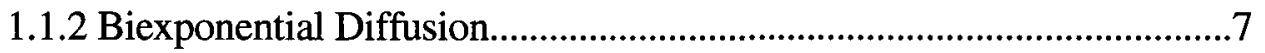

1.1.3 Intracellular and Extracellular Diffusion .........................................

1.2 Diffusion in Hydration Layers ……..........................................................12

1.3 Thesis Summary and Outline .............................................................................14

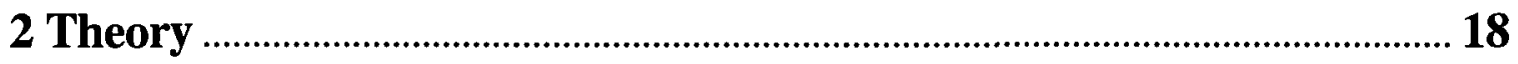

2.1 Principle of Nuclear Magnetic Resonance....................................................... 18

2.1.1 Classical Description ....................................................................... 18

2.1.2 Quantum Mechanical Description....................................................... 23

2.1.3 Statistical Distribution of Spin States.................................................. 25

2.2 Bloch's Equations and Relaxation................................................................... 26 
2.3 Spatial Encoding of the NMR Signal............................................................. 29

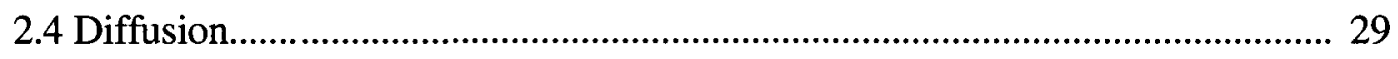

2.4.1 Diffusion Theory …………………………………......................... 30

2.4.2 Apparent Diffusion in Biological Tissues............................................. 32

2.4.3 Experimental Methods for Diffusion MR ......................................... 33

2.5 The Physics of $\mathrm{T}_{2}$ Relaxation Mechanism …….............................................. 38

2.5.1 Basics of Dipole-Dipole Interaction ................................................ 39

2.5.2 Relaxation and Molecular Motion........................................................ 41

2.5.3 NMR Studies of Water in Biological Systems..................................... 44

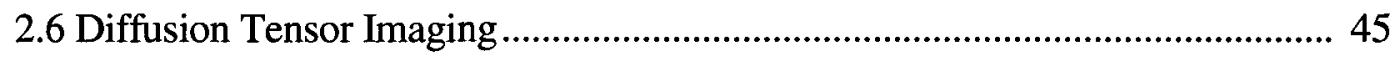

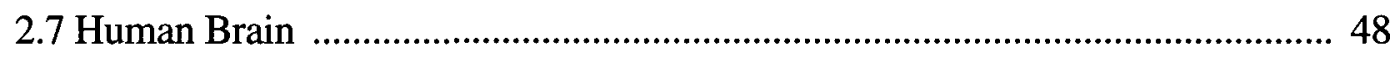

2.7.1 Microstructural Organization ............................................................. 48

2.7.2 Macrostructural Organization............................................................ 50

2.8 Tissue Water Model ................................................................................... 52

2.8.1 Hydration Layers .......................................................................... 54

3 Noise Correction …………………………………………………………………...61

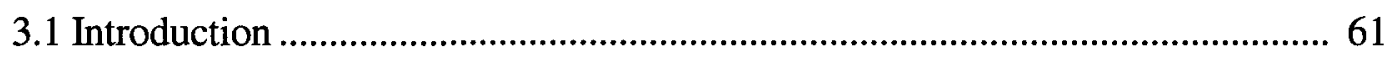

3.2 PDF for MR Magnitude Image Pixel Intensities ............................................. 63

3.2.1 The Rician Distribution ........................................................................ 63

3.2.2 The Moments of the Rician Distribution............................................ 66

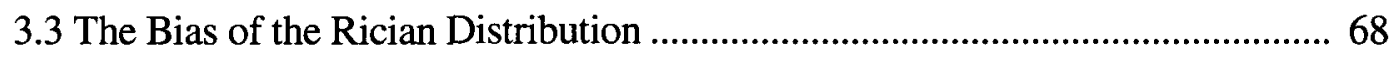

3.3.1 Review of Rician Bias Correction Techniques ................................. 68 vii 
3.3.2 Koay and Basser Rician Bias Correction ........................................... 70

3.3.3 Improved Rician Bias Correction Technique.........................................72

3.3.4 Validation of the Linear RB Correction Technique using

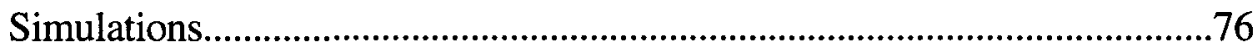

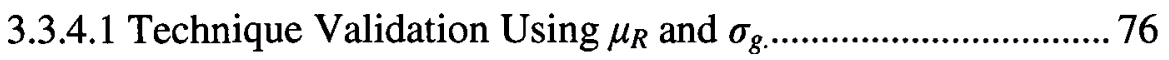

3.3.4.2 Convergence Criteria for the Linear RB Correction

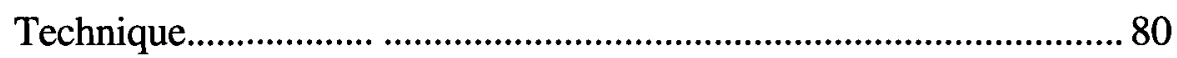

3.3.4.3 Linear RB Correction Using Nearest Neighbour

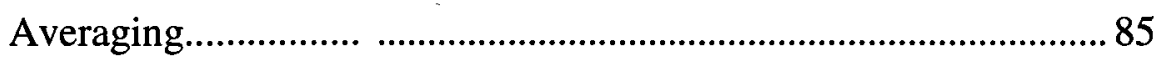

3.4 Rician Bias Correction Technique for Magnitude Averaged Signals ................ 88

3.4.1 The PDF of Magnitude Averaged Signals ......................................... 89

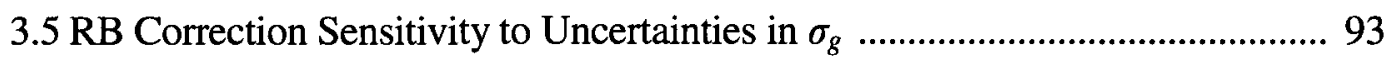

3.6 Validation of the Rician Bias Correction Using a Water Phantom ................... 94

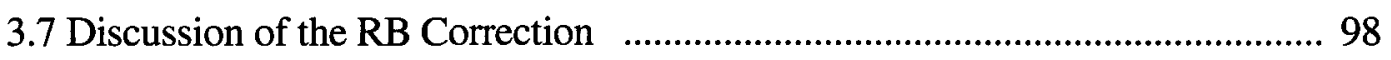

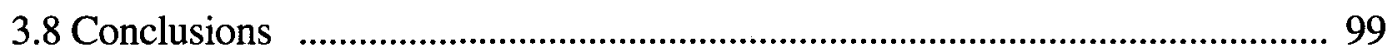

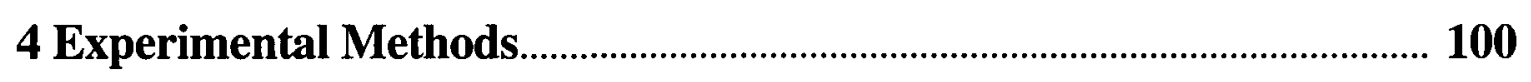

4.1 MR Experimental Protocol ............................................................................. 100

4.1.1 $\mathrm{T}_{2}$ Measurements ...................................................................... 100

4.1.2 Diffusion Measurements .................................................................... 101

4.1.3 Subjects and Slice Location.............................................................. 102

4.2 Decay Curve Analysis ............................................................................. 105

4.3 Water Phantom Measurements ............................................................... 106

viii 


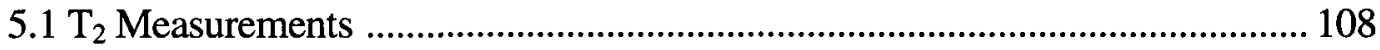

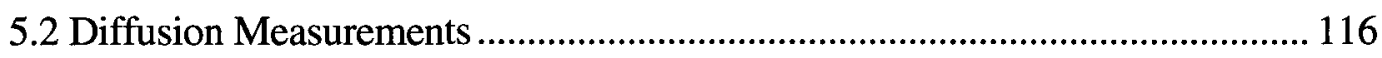

5.2.1 Diffusion Parallel to the Axons in the SCC and the GCC ................ 118

5.2.2 Diffusion Perpendicular to the Axons in the SCC and the GCC ...... 122

5.2.3 Diffusion Orientation Dependence in the SCC and the GCC ........... 126

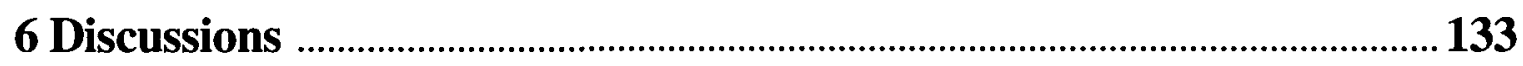

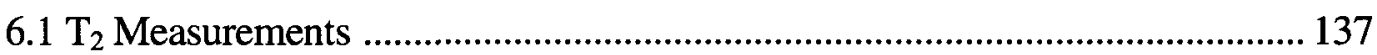

6.1.1 $\mathrm{T}_{2}$ Decay Curve Analysis .................................................................. 139

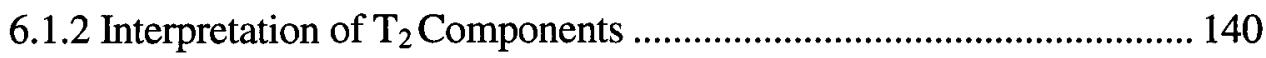

6.1.2.1 The Short $T_{2}$ Component...................................................... 142

6.1.2.2 The Long $T_{2}$ Component .................................................... 144

6.1.2.3 The Intermediate $T_{2}$ Component ....................................... 145

6.1.3 Hydration Layer and Disorder Zone Model ........................................ 146

6.1.4 Intracellular vs Extracellular............................................................... 149

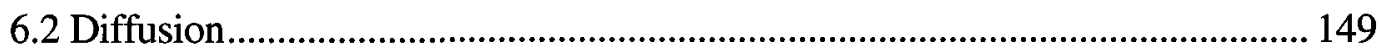

6.2.1 Experimental Protocol ..................................................................... 149

6.2.2 The Diffusion Decays ....................................................................... 151

6.2.2.1 Rician Bias Correction........................................................... 153

6.2.3 The Hydration Layers .......................................................................... 154

6.2.4 Diffusion in the Corpus Callosum....................................................... 157

6.2.4.1 Parallel Diffusion ................................................................... 157 
6.2.4.2 Perpendicular Diffusion........................................................ 159

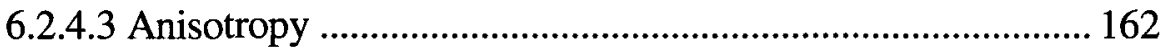

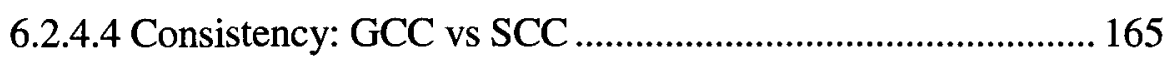

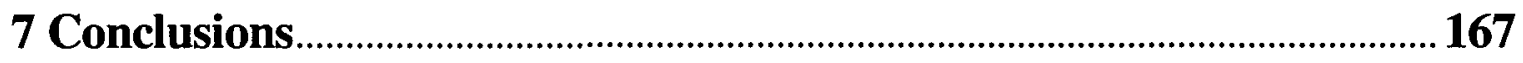

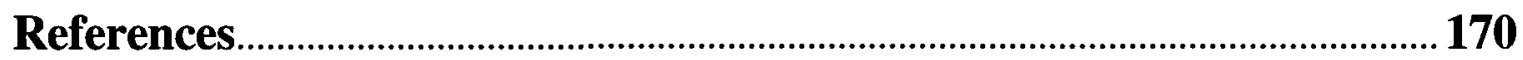




\section{List of Tables}

Table 3.1: Estimates of $\theta$, as a function of the correct values, were obtained using: 1) the method proposed by Gudbjartsson and Patz (column 3), 2) the binomial expansion implemented by Nezamzadeh with 8 terms included (column 4) and 3) Eq. (3.39) where exact values for $\mu_{R}$ and $\sigma_{g}$ are used to calculate the correction (column 5)

Table 3.2: RB correction and $\tilde{A}_{j}$ values obtained using Eq. (3.39) with $\left\langle M_{j}\right\rangle_{R n}$ set to the true mean, $\mu_{R}$, for given values of $A:$ a) $A=0.00$, b) $A=0.0001$, c) $A=$ 0.001 , as a function of the number of terms in the binomial expansion used to calculate the correction

Table 3.3: Values of $\tilde{A}_{j}$ and $A_{K, B}$ calculated using the true mean, $\mu_{R}$, for values of $A$ ranging from 0.00 to 3.00 The convergence criteria for this case were set such that terms were added to the correction until either a term less than $1 \times 10^{-14}$ was encountered or the number of terms exceeded $90,000,000$ 
Table 3.4: Values of $\tilde{A}_{j}$ and $A_{K, B}$ calculated using the true mean, $\mu_{R}$, for values of $A$ ranging from 0.00 to 3.00 The convergence criteria for this case were set such that terms were added to the correction until either a term less than $1 \times 10^{-9}$ was

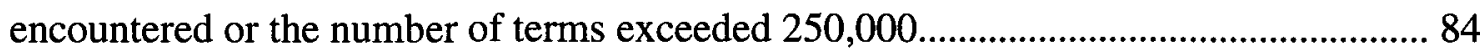

Table 3.5: $\left\langle\tilde{A}_{j, 1, n}\right\rangle_{R}$ values for $A$ ranging from 0 to 3 for several $n$ values and NEX $=1$.

For all simulations $\sigma_{g}$ was set to 1

Table 3.6: Estimates of the PDF mean for $A$ ranging from 0 to 3 and $\mathrm{NEX}=1,3,6$ and 9

Table 3.7: $\left\langle\tilde{A}_{j, 1, n}\right\rangle_{R}$ values for selected $n \times$ NEX combinations and signal values $A$ ranging from 0 to 3 . For all simulations $\sigma_{g}$ was set to 1 92

Table 3.8: Percent error for $\left\langle\tilde{A}_{j, 19}\right\rangle_{R}$ for various amounts of error in $\sigma_{g}$ for $A$ values that ranged from 0 to 2 94

Table 5.1: Signal fraction, $f_{i}$, and $T_{2}(i)$ transverse relaxation time estimates associated with each water fraction, $i$, for (a) the SCC and (b) the GCC of the 51 year old volunteer. 110

Table 5.2: Signal fraction, $f_{i}$, and $T_{2}(i)$ transverse relaxation time estimates associated with each water fraction, $i$, for (a) the SCC and (b) the GCC of the 36 year old volunteer..

Table 5.3: Signal fractions and $T_{2}$ relaxation times from trial 7 for the 51 year old subject reported in Table 5.1a with and without an extra set of echoes. The 93echo data set was acquired using the standard protocol and the 124-echo data 
set included the same 93 echoes plus an additional 31 echoes which extended the decay to $518.4 \mathrm{~ms}$

Table 5.4: Experimental results for the SCC of the 51 year old subject with diffusion sensitization in the LR direction for uncorrected (a) and corrected (b) normalized signal decay curves including the mean and standard deviation (SD) for the 9 trials

Table 5.5: Experimental results for the SCC of the 36 year old subject with diffusion sensitization in the LR direction for uncorrected (a) and corrected (b) normalized signal decay curves including the mean and standard deviation (SD) for the 9 trials

Table 5.6: Experimental results for the GCC with diffusion sensitization in the LR direction. Only the corrected normalized signal decay curves including the mean and standard deviation (SD) for the 9 trials are reported here for (a) the 51 year old subject and (b) the 36 year old subject

Table 5.7: Experimental results for the SCC of the 51 year old subject with diffusion sensitization in the IS direction for uncorrected (a) and corrected (b) normalized signal decay curves including the mean and standard deviation (SD) over the 8 trials 123

Table 5.8: Experimental results for the SCC of the 36 year old subject with diffusion sensitization in the IS direction for uncorrected (a) and corrected (b) normalized signal decay curves including the mean and standard deviation (SD) over the 8 trials 124 
Table 5.9: Experimental results for the GCC with diffusion sensitization in the IS direction. Only the corrected normalized signal decay curves including the mean and standard deviation (SD) for the 8 trials are reported here for (a) the 51 year old subject and (b) the 36 year old subject

Table 5.10: Fast and slow diffusion coefficients for the SCC of (a) the 51 and (b) the 36 year old subjects, respectively, reported as mean $\pm \mathrm{SD}$ along with their corresponding signal fractions, also reported as mean $\pm \mathrm{SD}$, derived from the biexponential fit for $\alpha=0-90^{\circ}$

Table 5.11: Fast and slow diffusion coefficients for the GCC of (a) the 51 and (b) the 36 year old subjects, respectively, reported as mean $\pm \mathrm{SD}$, along with their corresponding signal fractions, also reported as mean $\pm \mathrm{SD}$, derived from the biexponential fit for $\alpha=0-90^{\circ}$

Table 6.1: Composition of adult human brain. The total protein content represent 8$10 \%$ and the total lipid content is about $10 \%$ of the total fresh weight 148 


\section{List of Figures}

Figure 2.1: Precession of the magnetic moment $\mu$ with angular velocity $\omega_{0}$ about the static magnetic field $\boldsymbol{B}_{0}$

Figure 2.2 The motion of $\boldsymbol{M}$ in the laboratory frame showing the components along the $z$ axis and in the $x y$ plane.

Figure 2.3 a) The available orientations for $J$ and the corresponding values of $m_{z}$; b) Zeeman effect associated with the transition from the lower state to the upper state as a result of absorption of a quantum of $z$ axis and in the $x y$ plane.

Figure 2.4: The resultant FID signal induced by the $x y$ component of the sample magnetization.

Figure 2.5: The diffusion sequence PGSE with the basic components; the $90^{\circ}$ pulse, the $180^{\circ}$ pulse and both diffusion gradients 
Figure 2.6: A schematic representation of the organization of the mosaic structure of the lipid bilayer which forms the membrane matrix. Globular protein molecules are partially embedded in the membrane, and partially extending from it

Figure 2.7: Three-layer model of ordering of water near polar surfaces ..................................59

Figure 3.1: The Rician PDF for the magnitude image versus SNR ………………....................65

Figure 3.2: Estimates of $\theta$ plotted versus the correct values. The $\tilde{\theta}$ values were obtained by: 1 ) setting $\tilde{\theta}$ equal to $\mu_{R} / \sigma_{g}$ (solid line), 2) using the RB reduction method proposed by Gudbjartsson and Patz (dotted line) and 3) using Eq. (3.39) to calculate the exact RB correction (dashed line) for the case where $\mu_{R}$ and $\sigma_{g}$ are both known exactly. 78

Figure 3.3: Simulated PDFs for before (solid line) and after (dotted line) RB correction using the binomial expansion method based on Eq. (3.39)

Figure 3.4: Simulated PDFs for before (solid line) and after (dotted line) RB correction using the method proposed by Gudbjartsson and Patz .

Figure 3.5: PDFs for $A=0$ and $\mathrm{NEX}=1,3,6$ and 9 90

Figure 3.6: Semi-logarithmic plot of normalized diffusion decay curves for a water phantom before and after RB correction clearly showing the effect of Rician bias on the quickly decaying signals

Figure 3.7: Corrected and uncorrected diffusion decay curves for a water phantom plotted as SNR vs. $b$-value. The corrected decays were calculated using 9 and 225 nearest neighbor averages. 97

Figure 4.1: Sagittal view showing the slice of interest, SI oriented parallel to the reference central slice, $\mathrm{SC}$ 
Figure 4.2: a). Axial $F A$ maps showing the degree of anisotropy in each voxel. b). A color-coded white matter fiber map generated on the basis of fractional anisotropy and eigenvector information.

Figure 5.1: A typical $T_{2}$-decay curve for the SCC (triangles) along with the baseline noise (squares). Note that the signal intensity, expressed in arbitrary units (a.u.), remains well above baseline noise values for all echoes.

Figure 5.2: Typical $T_{2}$ decay curves consisting of signal intensity (in arbitrary units) versus echo time, $T E$, for the 51 year old subject acquired in the same imaging session. Two sets of 32 echoes are shown in the figure corresponding to echo spacings of 8.1 and $16.2 \mathrm{~ms}$. In a) the decay is shown on a linear plot and in b) the same data are shown on a semi-logarithmic plot

Figure 5.3: A typical plot of the residuals from a tri-exponential fit of a $T_{2}$ decay 115

Figure 5.4: Semi-logarithmic plot of SNR vs. $b$-values measured in the LR (diamonds) and IS (circles) directions for a 9-pixel ROI located within the SCC of the 51 year old subject before and after RB correction (open and solid symbols, respectively)

Figure 5.5: Normalized, RB corrected diffusion attenuation curves from the SCC of the 51 year old subject for $\alpha=0^{\circ}$ to $90^{\circ}$.

Figure 5.6: Variations of $D_{\text {fast }}$ and $D_{\text {slow }}$ plotted as a function of $\alpha$. 130

Figure 5.7: Changes in $f_{\text {fast }}$ and $f_{\text {slow }}$ plotted as function of $\alpha$ for the SCC (a) and the GCC (b). The error bars refer to the standard deviation of the fitted parameter obtained from the Marquardt-Levenberg fit 


\section{List of Symbols}

$A \quad$ the true magnetic induction signal

$A_{c} \quad$ true, noiseless complex MR signal

$A_{I} \quad$ imaginary component of the true MR signal

$A_{R} \quad$ real component of the true MR signal

$\tilde{A}_{G P} \quad$ Gudbjartsson and Patz estimator for $A$

$\tilde{A}_{j} \quad$ estimator for $A$ corresponding to $\operatorname{pixel} j$

$A_{K, B} \quad$ Koay and Basser theoretical value of $A$

$A D C \quad$ apparent diffusion coefficient

$\boldsymbol{B}_{0} \quad$ static magnetic field

$B_{1} \quad$ rf excitation pulse applied to spin system

D self-diffusion coefficient of water

$D_{\text {fast }} \quad$ fast diffusion coefficient

$D_{\text {slow }} \quad$ slow diffusion coefficient 


\begin{tabular}{|c|c|}
\hline $\bar{D}$ & diffusion tensor \\
\hline$E$ & dipole-dipole energy interaction \\
\hline$E_{+\frac{1}{2}}$ & $E_{+\frac{1}{2}}=-\gamma \frac{\hbar}{2} B_{0}$, lower state energy \\
\hline$E_{-\frac{1}{2}}$ & $E_{-\frac{1}{2}}=\gamma \frac{\hbar}{2} B_{0}$, upper state energy \\
\hline$E\left[M^{\nu}\right]$ & $v^{\text {th }}$ moment of the Rician PDF \\
\hline$F A$ & fractional anisotropy \\
\hline G & diffusion gradient amplitude \\
\hline$H_{D}$ & dipole-dipole Hamiltonian \\
\hline$H_{D}^{0}$ & secular term of the Hamiltonian $H_{D}$ \\
\hline $\boldsymbol{I}$ & identity matrix \\
\hline$I_{0}$ & Oth order modified Bessel function of the first kind \\
\hline$I_{1}$ & first order modified Bessel function of the first kind \\
\hline$I^{+}$ & raising operator \\
\hline$I^{-}$ & lowering operator \\
\hline$J$ & nuclear angular momentum \\
\hline$J_{z}$ & $z$-axis component of $J$ \\
\hline$J(\omega)$ & spectral density function \\
\hline$M$ & magnetization of spin system \\
\hline$M_{x y}$ & transverse magnetization component \\
\hline$M_{z}$ & longitudinal magnetization component \\
\hline$N$ & number of simulated Rician distributed data points \\
\hline$N_{A}$ & Avogadro's number \\
\hline
\end{tabular}




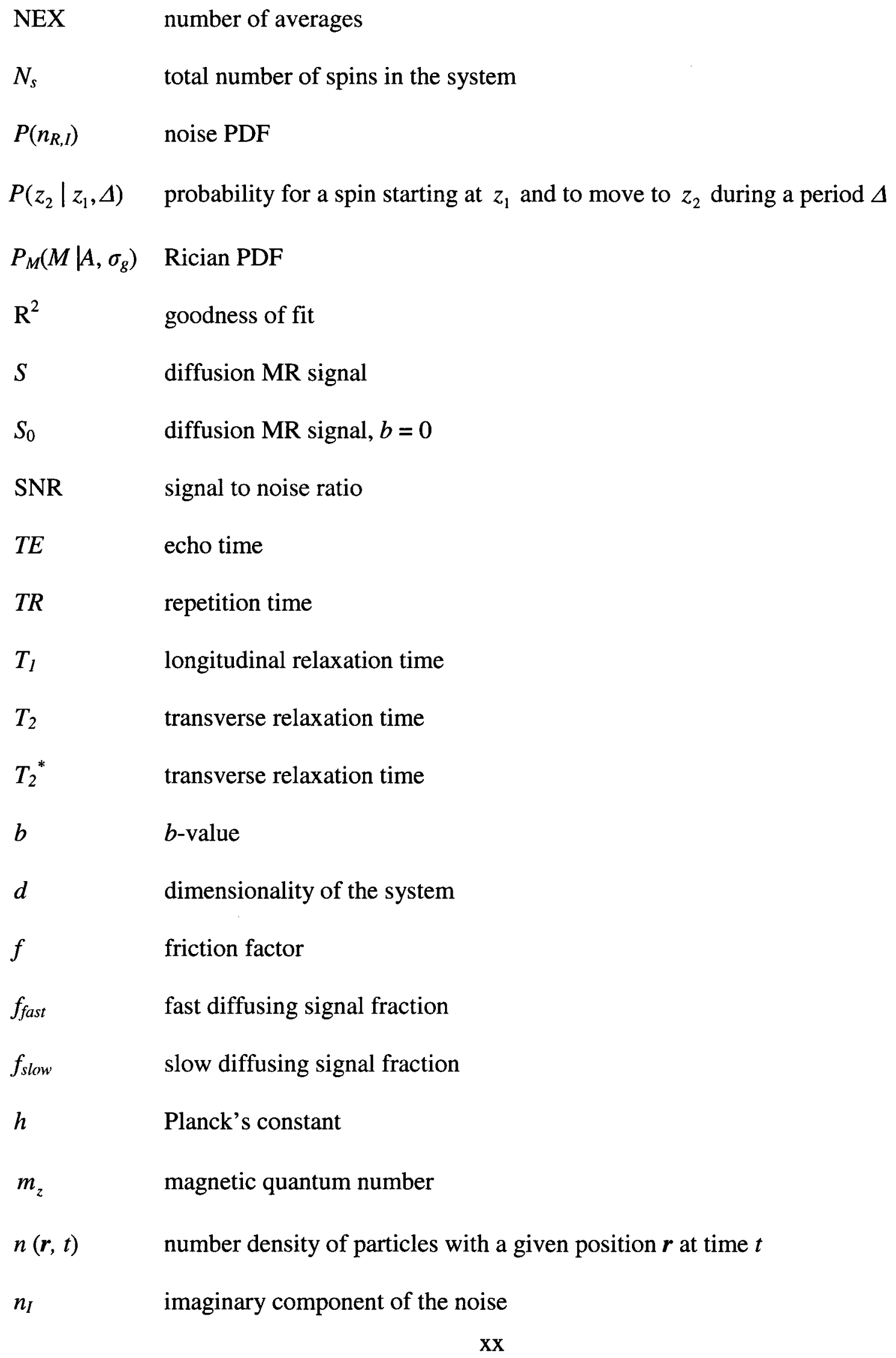




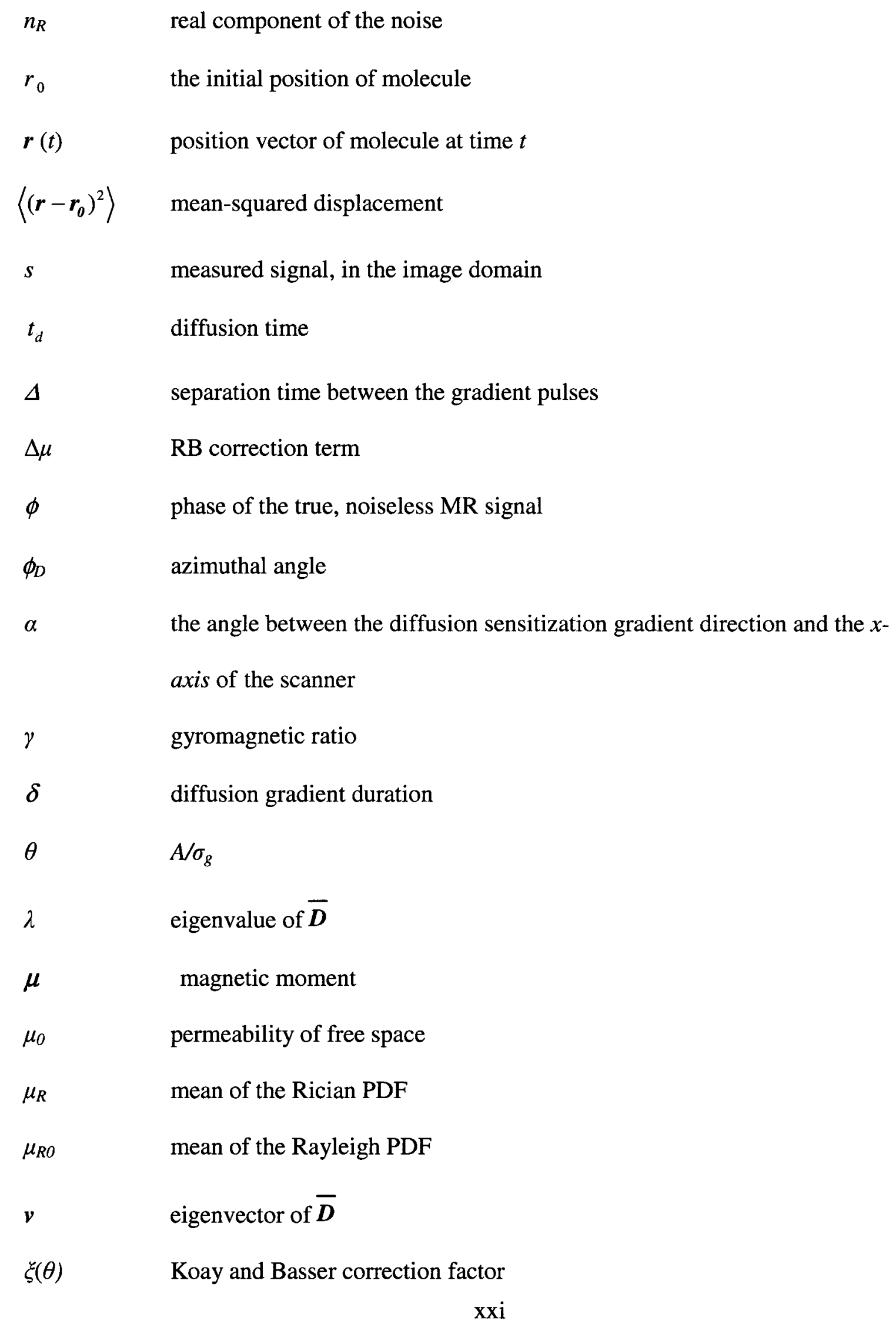




$\begin{array}{ll}\rho & \text { density of spins } \\ \sigma_{g} & \text { standard deviation of the noise } \\ \sigma_{R} & \text { standard deviation of the Rician PDF } \\ \tau & \text { torque } \\ \tau_{\mathrm{c}} & \text { correlation time } \\ \varphi & \text { residual phase-shift of spins } \\ \varphi_{1} & \text { phase-shift induced by first diffusion gradient } \\ \varphi_{2} & \text { phase-shift induced by second diffusion gradient } \\ \omega & \text { angular velocity } \\ \omega_{0} & \text { Larmor frequency }\end{array}$




\section{Chapter 1}

\section{Introduction}

It is amazing to recognize that more than five decades after the discovery of the phenomenon of nuclear magnetic resonance (NMR), the evolution of the magnetic resonance imaging (MRI) technology is still proceeding with considerable speed. Furthermore, the technique of diffusion-weighted imaging (DWI), one of the most recent products of this evolution, provides a unique form of contrast in MR images that is of unquestioned diagnostic value [1].

Although DWI images of the brain were firstly obtained in the middle 1980 s, both in healthy subjects and in patients [2], this technique was not exploited clinically until the mid 1990s. Initially, hardware limitations of clinical MRI scanners made it difficult to obtain reliable diffusion-weighted images since long acquisition times were required and the resulting images were consequently sensitive to macroscopic motion, such as breathing, head movement or brain motion induced by the pulsatile motion of blood in the brain during the cardiac cycle 
[3]. Nowadays, with better MRI scanners, it is possible to collect a set of diffusion weighted images covering the whole brain in less than a second, virtually freezing all macroscopic motion.

One of the reasons why DWI has attracted so much interest is that it provides access to tissue dynamics at the microscopic scale, even though the detected overall signal is from tissue volumes in the millimeter range (i.e. macroscopic). This is an example of a multi-scale process whereby information about the system dynamics on the microscopic scale can be inferred from measurements performed on a macroscopic scale. Einstein used a similar multiscale approach in developing his molecular theory of heat and presented it as a way to indirectly validate the existence of molecules and atoms [4].

Diffusion of molecules in a homogeneous liquid can be characterized by a physical constant called the self-diffusion coefficient, $D$, which is related to the root mean square displacement, $\Delta r$, of the molecules over a given diffusion time, $t_{d}$, via the Einstein equation:

$$
\Delta r=\sqrt{2 d D t_{d}}
$$

where $d=1,2$ or 3 when diffusion in 1,2 or 3- dimensions is being considered, respectively.

MRI techniques can be made sensitive to diffusion in one dimension, known as the diffusion direction, by inserting appropriate magnetic field gradient pulses into a standard MRI pulse sequence. The diffusion direction, which can be adjusted to any desired direction, is determined by the orientation of these extra diffusion sensitizing gradient pulses. Images with different levels of diffusion sensitization can be acquired by varying the gradient pulse magnitude.

In pure liquids, the signal decay as a function of the diffusion weighting, is exponential and can be expressed as: 


$$
S / S_{0}=\exp (-b D)
$$

where $b$ is a measure of diffusion sensitization and $S$ and $S_{0}$ are the signals at $b$ and $b=0$, respectively. The $b$-value is a complicated function of the amplitude and duration of the diffusion-sensitizing gradients (see Eq. 2.49).

Since the diffusion coefficient of water in brain tissue at body temperature is about $1 \times$ $10^{-3} \mathrm{~mm}^{2} / \mathrm{s}$, Eq. (1.1) predicts that about two thirds of water molecule displacements due to diffusion in any given direction will be $\sim 10 \mu \mathrm{m}$ or less for $t_{d} \approx 50 \mathrm{~ms}$, which is typical of the diffusion times currently used in DWI. This is well below the typical MRI image resolution of $\sim 1 \mathrm{~mm} /$ dimension. Only for much shorter diffusion times (i.e. $t_{d}<1 \mathrm{~ms}$ ) will the measured diffusivity reflect the local intrinsic viscosity. Unfortunately, it is not currently possible to measure diffusion for $t_{d}<1 \mathrm{~ms}$ due to hardware limitations. For longer $t_{d}$ 's compartmentalization and membrane permeability are generally considered to have important effects on the measured diffusion coefficient. The implicit observation of these molecular displacements via measured diffusion coefficients provides information that reflects aspects of tissue microstructural architecture such as cell geometry and orientation [5]. Water diffusion in brain tissue is different from that in bulk water and can be regarded as an intrinsic marker of local cellular structure. Changes in the measured diffusion coefficient from the normal behaviour for a given cell type can often be associated with different physiological and/or pathological states.

A great deal of research has been done on DWI since it was first introduced and much progress has been made. However, there is still considerable debate regarding the nature of the water molecular dynamics at the microscopic scale that determine the observed macroscopic behavior. Much of the emphasis to date has been on a model that considers 
intracellular and extracellular diffusion separately, with the axonal plasma membrane and the myelin sheath being considered as reflective/semi-permeable barriers. Unfortunately, this approach leads to inconsistencies inherent to this model. In this thesis, a different model is considered where the structuring of the water molecules into hydration layers around the macromolecular structures of both the intracellular and extracellular spaces is considered to be the most important perturbing effect. These concepts are introduced in more detail in the rest of this chapter and an outline of the rest of the thesis is given at the end.

\subsection{Overview of the MR Diffusion Literature}

\subsubsection{The Apparent Diffusion Coefficient}

As a departure from earlier studies of water diffusion in tissues, where efforts were made to depict the elementary diffusion process [6-8], it was suggested that the diffusion coefficient, $D$, should be replaced for tissue studies by a global parameter, known in the MR literature as the apparent diffusion coefficient or the $A D C$. This was felt to be necessary since the measured diffusion coefficient is not a true measure of 'intrinsic' diffusion, but rather it reflects the complex molecular displacements that occur in biological tissues due to interactions of water molecules with cellular structures and processes for a given diffusion time. Furthermore, the diffusion signal measured for a tissue voxel, which normally contains thousands of cells and tissue components, is an overall average of many different effects and microscopic environments. With this approach it is implicitly assumed that the diffusion decay is exponential. Modeling is necessary to make inferences about the microscopic motions 
from the observed signals with their macroscopic resolution. The $A D C$ concept has been widely adopted in the medical MR literature.

The first important application of diffusion MRI emerged in the early 1990s when it was discovered that stroke can be detected in its acute phase using DWI [9], although the exact mechanism responsible for the observed $A D C$ drop remains poorly understood. Around the same time, Moseley et al [10] noticed that water diffusion is anisotropic for normal white matter in cat brain and spinal cord whereas diffusion in grey matter is isotropic. Diffusion anisotropy in humans in vivo has been observed in the spinal cord [11], sciatic nerve [12], tibial nerve [13] and the corpus callosum [14]. In white matter, the diffusion anisotropy is a consequence of the organization of the tissue into tracts containing myelinated axonal fibers running parallel to each other. It is known that diffusion in the direction of these fibers is faster than in the perpendicular direction, although a complete explanation of how the neuronal structures lead to this anisotropy remains elusive. This property of white matter tissue generates orientation-dependent contrast in diffusion weighted images that can be exploited to track axonal tracts in the brain using Diffusion Tensor Imaging (DTI) and Tractography [15, 16].

Despite the recent widespread use of DWI and many published research studies on the subject, a clear relationship between the measured diffusion coefficients and known histological and physiological parameters has not yet been clearly established. More specifically, there is still uncertainty concerning how changes in tissue microstructure relate to observed $A D C$ variations. Since the early days, the mechanism underlying brain tissue water diffusion at the molecular level has been a popular subject of research. The first NMR study of water diffusion in tissue appears to have been made by Maltsev [17] who reports diffusion 
coefficients for frog liver, muscle, skin and kidney (at an unspecified temperature) between 0.8 $\times 10^{-3}$ and $1.8 \times 10^{-3} \mathrm{~mm}^{2} / \mathrm{s}$. In the early-to-mid $1970 \mathrm{~s}$ there was great interest in understanding the state of ions and water in cytoplasm. Hansen [18] reported that the $A D C$ values for ions and water in tissue are reduced by a factor of 2-5 when compared to $D$ of bulk water, while the translational diffusion of water in excised brain tissue appeared to be hindered to a greater extent than in excised muscle tissue.

It was initially believed that the overall low diffusivity in the intracellular space could be explained by restriction effects caused by cellular membranes [19] and by scattering or obstruction effects from cellular macromolecules [20-22], it is now commonly believed that there is more to the story. Simulations have shown that the effects of compartmentalization on the intracellular diffusion behaviour can be explored by measuring the diffusion coefficient as a function of the diffusion time, $t_{d}[23,24]$. The same effect has also been reported for cell suspensions [25]. Variation of the $A D C$ with $t_{d}$ ranging from 35 to $350 \mathrm{~ms}$ has also been obtained in excised brain tissue, [26] and edematous brain tissue [27]. In a study of diffusion displacements measured at several different diffusion times in rat spinal cord no clear evidence of restriction was reported [28]. Hindered diffusion may be a more appropriate term than restricted diffusion in brain tissue since membranes have a finite permeability to water. Although the membranes likely hinder the water diffusion process, they are highly permeable to water, either passively or through transporters such as the specific aquaporin channels which have been found to be abundant in the brain [29].

The inability to measure the effects of restricted diffusion does not mean that it is not present. Two possible explanations for why it has not been observed are: 1) diffusion time dependent effects may be more apparent over a larger range of b-values than has 
conventionally been used and 2) the diffusion times used in most studies to date were not short enough. Simulation studies predict that for diffusion perpendicular to partially permeable membranes, the $A D C$ derived from a mono-exponential fit at low $b$-values will start to change markedly for $t_{d}<4 \mathrm{~ms}$ [30]. Consequently, probing in vivo restricted diffusion on a clinical MRI system is inherently difficult at present due to hardware limitations.

\subsubsection{Biexponential Diffusion}

It is well known that molecular displacements due to diffusion for a pure liquid, such as water, follow a Gaussian distribution. MR diffusion measurements in such systems yield exponential diffusion decays. Many studies have experimentally established that the diffusion-sensitized MR signal attenuation for water in brain tissue as a function of the $b$-value is not well described by a single exponential decay such as would be observed for normal "Gaussian" diffusion in an unrestricted, homogenous medium. Non-Gaussian diffusion has also been observed in neuronal tissues using strong diffusion weighting that sensitized the MR signal to molecular displacements of less than $2 \mu \mathrm{m}$ [31]. This approach revealed a pool of water molecules with highly anisotropic and reduced diffusion, which was attributed mainly to water located in the intra-axonal space [32]. If a significant portion of the signal observed at high $b$-values originates from restricted motion of intra-axonal water, new information about axonal morphology could be obtained that would potentially improve the understanding of white matter diseases and their development.

Numerous observations have shown that the signal decay is better described by a biexponential function, presumably corresponding to diffusion in two distinct water pools $[26$, $31,33]$ : 


$$
S / S_{0}=f_{\text {fast }} \exp \left(-b D_{\text {fast }}\right)+f_{\text {slow }} \exp \left(-b D_{\text {slow }}\right)
$$

where $D_{\text {fast }}$ and $D_{\text {slow }}$ are the diffusion coefficients associated with the fast and slow diffusing water pools, respectively and $f_{\text {fast }}$ and $f_{\text {slow }}$ are their corresponding signal fractions. The two water pools must also be in slow exchange such that exchange between the two pools is negligible in order for such a biexponential decay to be observed. For the case of "intermediate exchange", the above biexponential mathematical model remains valid; however, the parameters given above must be replaced by more complex quantities that take into account the residence time of the molecules in the two compartments relative to the measurement time [34].

Niendorf et al [31] reported values for $D_{\text {fast }}=(8.24 \pm 0.30) \times 10^{-4} \mathrm{~mm}^{2} / \mathrm{s}, D_{\text {slow }}=(1.68$ $\pm 0.10) \times 10^{-4} \mathrm{~mm}^{2} / \mathrm{s}$ with $f_{\text {fast }}=0.80 \pm 0.02$ and $f_{\text {slow }}=0.17 \pm 0.02$ in the rat brain in vivo, with $b$-values up to $10,000 \mathrm{~s} / \mathrm{mm}^{2}$ using this biexponential model. Despite the striking agreement in the literature of reported human brain tissue $A D C$ values obtained with different measurements using high $b$-values [35-38], questions regarding the correct interpretation of the signal decay still arise and are not easily answered.

\subsubsection{Intracellular and Extracellular Diffusion}

It was initially suggested that intracellular diffusion should be slower than extracellular diffusion since protein concentration is higher inside the cell $[26,31]$. Yet measurements have shown that the volume fractions of the two water pools obtained using the biexponential model do not correspond at all with anatomy; the extracellular compartment represents about $20 \%$ of the total volume [39] whereas the measured fraction for the fast diffusion coefficient, which is conventionally associated with the extracellular compartment, is consistently reported to be 70 
- $80 \%[35,40]$. This discrepancy exists even after taking into account differences in $T_{2}$ relaxation contributions between these compartments.

Several theoretical models, which have been developed assuming highly idealized intracellular and extracellular compartments separated by semi-permeable membranes, have been proposed in the literature $[24,30,41]$. Based on these models, restricted intracellular and extracellular diffusion has been suggested as an explanation for the observed non-exponential diffusion decay in axons in vivo. With these models the cells are, in general, considered to be regularly organized geometrical structures and use a large number of parameters. While such studies are very informative and useful, they are not realistic enough to validate the twocompartment hypothesis for in vivo diffusion data.

Furthermore, numerical simulations based on known anatomy have shown that the assignment of signal fractions obtained from biexponential fits of the diffusion decay to extracellular and intracellular spaces does not give the correct volume fractions. They have also shown that such pseudo-biexponential diffusion behaviour can be seen to arise solely from the intracellular compartment $[42,43]$. Micro-imaging studies in isolated Aplysia neurons also revealed more than one $A D C$ value, suggesting the presence of several intracellular compartments. These results also imply that the intrinsic water diffusivity in axons may differ substantially from that in neuronal bodies. In addition, Schwarcz et al [44] still observed a biexponential signal decay in human erythrocyte samples with disintegrated membranes and negligible extracellular space indicating that compartmentalization is not essential in these diffusion measurements.

In contrast, the tortuosity coefficient, $\lambda$, has been used to try to explain experimental observations attributed to the extracellular space. Decreased diffusion path lengths caused by 
obstructing cells are considered to be a potential source of diffusion reduction within the extracellular space [39]. Experiments using NMR in vitro, have also shown that the $A D C$ decreases when cellular density is increased [45]. However, the mechanism responsible for this is not obvious since the observed $A D C$ changes are linked to both changes in cell volume and the related changes to the extracellular space and it is difficult to untangle these two effects.

There is no need to have intracellular and extracellular compartments to observe a biexponential diffusion decay. But, if this hypothesis is accepted, to which "compartments" do the observed diffusion components correspond? There is also no definitive evidence that there are two compartments; there may be more, there may be only one or there may be no diffusive compartments at all. According to Assaf et al [46] diffusion data is best fitted with three exponentials rather than with two and Pfeuffer et al [47] indeed seems to find 3 peaks when they analyzed their diffusion data using a Laplace transform. Numerical simulations demonstrate that multiple components are not necessary in order to observe a multiexponential decay [48-50]. Several groups have also pointed out that the observed signal decay curve could possibly originate from a heterogeneous distribution of cell sizes [51].

There is already a large body of knowledge in the literature which suggests that restriction and hindrance phenomena may be more plausible as explanations for the nonexponentiality of the diffusion decay, yet experimental confirmation is hard to obtain. Furthermore, diffusion anisotropy must also be connected to the same restriction and hindrance effects [52] but no simple relationship has been established with the known microstructural elements [53]. Cell membranes have been regarded as the primary determinants of water restriction [54] and the degree of axonal packing and neural organization indeed affects diffusion measurements in white matter. However, no 
corresponding explanation for the observed biexponential diffusion decays in grey matter has been established. The relatively new CHARMED model [55], built on the assumption that water has both restricted and hindered behaviour characterized by normal distributions, still lacks in vivo validation, but shows some promise in the study of white matter structures.

Yablonskiy et al [56] developed a statistical model of the diffusion signal decay, based on the hypothesis that many diffusion water populations coexist with a resulting probability distribution of diffusion coefficients which is Gaussian. This result is quite complex and difficult to interpret as the average diffusivity is different in each tissue and appears to be independent of the tissue structure. In order to characterize the tissue heterogeneity, Bennett $e t$ al [57] have proposed the following stretched exponential equation for diffusion in tissues:

$$
S / S_{0}=\exp \left[-\left(b^{*} D D C\right)^{\alpha}\right]
$$

where $D D C$ stands for Distributed Diffusion Coefficient, and $\alpha$ is used to parameterize the tissue heterogeneity. Interestingly, $\alpha$ does not seem to vary with the diffusion direction. This observation, if it turns out to be valid, would have a practical advantage since measurements with only one diffusion gradient orientation would be sufficient. This would also mean that faster evaluations of tissue microstructure would be possible.

In summary, there are growing indications that many parameters may influence diffusion signal variation as discussed in this overview including water compartmentalization, restriction and hindrance in intracellular and extracellular spaces, and tissue heterogeneity. However, a model that successfully explains the experimental observations in terms of these effects has not yet been proposed. There is a need to look at the data in another way, for example to get closer to the complex reality of the biology. 


\subsection{Diffusion in Hydration Layers}

The origin of water diffusion behaviour in brain tissue must be reconsidered by reinforcing the peculiar properties of water in biological system. Despite the sophistication of current experimental and analysis tools, for example the statistical diffusion model [56], generalized diffusion tensors [58, 59], $q$-space imaging [60] and diffusion kurtosis [61], MR diffusion techniques are still going through a painful gestation period of interpretational controversy. And why not? Disappointingly, almost all of them neglect the chemical properties of tissue water.

The properties of water in biological systems have been studied for well over a century by a wide range of physical techniques, including NMR. Over the past two decades, it has become increasingly clear that water is an active component in all cells. The special properties of water molecules are necessary for the normal operation of biological mechanisms within cells. Conversely, these mechanisms and the contents of the cells have a profound impact on the water structure itself. This important reciprocal relationship is often overlooked.

Three years ago, Le Bihan [62] suggested the importance of layers of water molecules that are most hindered in their motion, such as a membrane-bound water pool. He proposed that the slow diffusion component extracted from the diffusion decay may correspond to water molecules trapped within a membrane-bound water network. To the best of our knowledge, no experimental evidence has been reported to directly support his suggestions.

There is experimental evidence regarding the state of water in biological systems and a significant portion of this evidence comes from the results of NMR studies. NMRspectroscopy studies have reported that the water resonance peak for aqueous DNA solutions is much broader than the signal from pure water due to increased ordering of the water and the 
formation of hydration shells around the DNA [63]. This was also confirmed for frog muscle $[64,65]$ and rat and rabbit nerves $[66,67]$ leading to the general conclusion that water in tissue exists in more than one fraction with one of the fractions corresponding to a well-defined and ordered water phase. There is also some evidence for oriented water in tendons and other connective tissues [68, 69]. In addition, nearly all of these studies found more than one relaxation time for water and the values were much lower than the values observed for free water.

The decreased diffusion coefficient in cells relative to diffusion in pure bulk water could be assigned to the obstruction effect of hydrated macromolecules [70, 71] and is not necessarily due to an overall decrease in molecular mobility. The value of the observed diffusion coefficient in aqueous protein solutions has been shown to depend on protein concentration since obstructions and water-protein interactions increase with macromolecular crowding and membrane hindrance effects. Recently, Colsenet et al [22] evaluated the water diffusion coefficients in cells by taking into account the obstruction of water by proteins and water-protein interactions. They predict that:

$$
D_{\text {cell }}=D_{b u l k} \frac{1}{1-\varphi\left(1-\frac{C_{\text {hydr }}}{C_{\text {bulk }}}\right)} \frac{1-\beta \varphi}{1+\frac{\beta \varphi}{2}}
$$

with

where $C_{h y d r}$ and $D_{h y d r}$ are the hydration water concentration and diffusion coefficient, respectively, $C_{b u l k}$ and $D_{b u l k}$ are the bulk water concentration and diffusion coefficient, respectively, (they report $D_{\text {bulk }}=1.95 \times 10^{-3} \mathrm{~mm}^{2} / \mathrm{s}$ at $20^{\circ} \mathrm{C}$ ) and $\varphi$ is the protein concentration. The $D_{\text {cell }}$ values can be seen to decrease with increasing protein concentration $\varphi$ and, as 
expected, for very low protein content $\left(1 \mathrm{~g}\right.$ of protein $/ 100 \mathrm{~g}$ of water), the $D_{\text {cell }}$ approaches the pure water self-diffusion coefficient $D_{b u l k}$.

The general remark that structured water has a biological role and membranes might give rise to this structured cellular water seems to come out through many studies of NMR spectral line widths, chemical shifts, and relaxation times of water protons in biological tissues, biopolymer systems [72, 73], protein solutions such as bovine serum albumin [74] and animal eggs [75], bacterial systems [76], lipid lamellar systems [77, 78] and many, many more.

Brain diffusion data at high $b$-values has not been properly exploited in the past due in part to hardware limitations and the fact that its quantification must be done with great care. In light of recent research involving the analysis of water behaviour in more complex tissue structures and developments in MR coil and gradient technology, a re-evaluation of the models used to interpret DWI decay data is required. To properly interpret this diffusion behavior for tissue water we are brought back to the lines of research considered many years ago but neglected in recent years. The diffusion decay data presented in this thesis will be interpreted in terms of a hydration layer model instead of the more common 2-compartment restricted/hindered diffusion approach.

\subsection{Thesis Summary and Outline}

\section{Statement of Originality:}

The two main original contributions presented in this thesis are: A) a novel explanation of MR diffusion using a hydration layer model and B) an improved method for removing 
Rician bias (RB) from the measured data. The presented work in both of these areas is summarized below.

\section{A. Hydration Layer Model}

The primary goal of this thesis was to propose a novel explanation for the observed diffusion properties of tissue water in human white matter in vivo. Traditionally, diffusion in white matter has been interpreted in the literature in terms of restricted diffusion models with two well defined compartments: the intracellular space and the extracellular space. With these models the water molecules are normally considered to diffuse as if they were in pure water until they meet a barrier, such as a cell membrane, which simply reflects the particle back in a very classical sense. However, these models have not been successful in accounting for all of the observed results; in particular, the observed signal fractions are inconsistent with this model. The effect on diffusion of the electrostatic interactions of water molecules with each other and with their surroundings, particularly with polar sidegroups of macromolecules which may be inside the cell or incorporated into the cell's membranes, are normally ignored but can be expected to have an important effect. These interactions lead to the formation of hydration layers around macromolecules and along membrane surfaces and cause the water in the cell to behave more like a gel that a liquid. Incorporation of these considerations into the analysis has allowed developing novel explanations for: 1) why the measured fast diffusion component is smaller than the diffusion coefficient of free water, 2) why it is anisotropic, 3) the observed signal fraction values and 4) the anisotropy of the signal fractions.

In this thesis carefully measured sets of diffusion decays for the splenium and the genu of the corpus callosum of two healthy subjects in vivo are presented along with supporting $T_{2}$ 
relaxation time measurements. The diffusion decays were measured as a function of the angle between the diffusion direction and the long axis of the axon. All of these data are successfully interpreted in terms of the proposed hydration layer model.

\section{B. Rician Bias Correction}

A secondary goal of the thesis was to develop an improved method for removing the $\mathrm{RB}$ from the diffusion data. The diffusion decays were measured for $b$-values up to 10,000 $\mathrm{s} / \mathrm{mm}^{2}$. At these large $b$-values the decays are affected by RB. Without properly correcting the decays for these effects, quantitative analysis and interpretation of the decays is meaningless. Until recently, no reliable method existed for the removal of this bias. However, an RB correction method which is analytically exact, as long as accurate values for the mean and standard deviation of the signal probability density function are available, has recently been published. A method for implementing this method when only approximate values of the true mean and standard deviation are known has also recently been proposed. These novel techniques from the literature are reviewed in Chapter 3 and then extended to a more efficient algorithm which is appropriate for the data reported in this thesis.

Chapter 2 contains a review of all of the relevant background for the thesis other than the theory of noise in MR which is dealt with in Chapter 3 where a novel RB correction technique is explained and then extended to apply to the data reported in this thesis. Chapters 4,5 and 6 contain the Experimental Methods, Results and Discussion for the experiments performed, respectively. The major portion of this experimental work deals with diffusion decay measurements in the corpus callosum of healthy human volunteers in vivo. These experimental results are explained using a hydration layer model rather than the more 
traditional intracellular/extracellular approach. Supporting $T_{2}$ decay experiments are also presented and analyzed in terms of the same hydration layer model. These results are reviewed and summarized in the Conclusions chapter, Chapter 7 . 


\section{Chapter 2}

\section{Theory}

\subsection{Principles of Nuclear Magnetic Resonance}

\subsubsection{Classical Description}

The physics of Nuclear Magnetic Resonance, NMR, starts with the property of certain nuclei called "spin" $[79,80]$. Nuclei having non-zero spin, those with an odd number of protons and/or neutrons, include several that are biologically abundant, such as ${ }^{1} \mathrm{H},{ }^{13} \mathrm{C},{ }^{31} \mathrm{P}$ and ${ }^{23} \mathrm{Na}$. Modern radiological procedures are primarily concerned with the ${ }^{1} \mathrm{H}$ nucleus, which is simply an individual proton, because of its large NMR signal and high natural abundance in biological systems.

The charge of a proton can be considered to be distributed and rotating about a central axis as a result of a non-zero angular momentum. The spinning motion of the charge induces a 
local magnetic field, making each nucleus behave like a tiny magnet characterized by the magnetic moment, $\mu$. The magnetic field of the proton is parallel to the angular momentum and normal to the plane of charge circulation. In this case, $\mu$ is given by:

$$
\boldsymbol{\mu}=\gamma \boldsymbol{J},
$$

where $\gamma$ is the gyromagnetic ratio. For a proton, $\gamma \cong \frac{q}{2 m}$, where $m$ and $q$ are the proton mass and charge. Note that vectors are indicated by a bold font throughout the thesis.

An external magnetic field, $\boldsymbol{B}_{0}$, will exert a torque on a magnetic moment, causing the angular momentum to change at a rate given by:

$$
\tau=\frac{d J}{d t}=\mu \times \boldsymbol{B}_{0},
$$

or using Eq. (2.1) :

$$
\left|\frac{d J}{d t}\right|=\gamma J B_{0} \sin \theta
$$

where $\theta$ is the angle between $\boldsymbol{B}_{0}$ and $\mu$. Thus, during an infinitesimal time $d t$, the angular momentum will change by an amount $d \boldsymbol{J}$ corresponding to a rotation through an angle $d \phi$ in the plane perpendicular to $\boldsymbol{B}_{0}$. As shown in Fig. 2.1, this angle is given by:

$$
d \phi=\frac{d J}{J \sin \theta}=\frac{\gamma J B_{0} \sin \theta d t}{J \sin \theta}=\gamma B_{0} d t .
$$

The frequency of precession of the spin therefore is given by:

$$
\omega_{0}=\frac{d \phi}{d t}=\gamma B_{0} .
$$

Since each nucleus is spinning, the torque on it produced by the magnetic field prevents it from actually aligning with the applied magnetic field. Instead it precesses around the 
magnetic field. The behaviour is analogous to a gyroscope in a gravitational field. Eq. (2.5) states that the precessional frequency, which is known as the Larmor frequency, is directly proportional to the magnetic field strength. This is the fundamental concept in MRI and is one from which almost everything else can be derived.

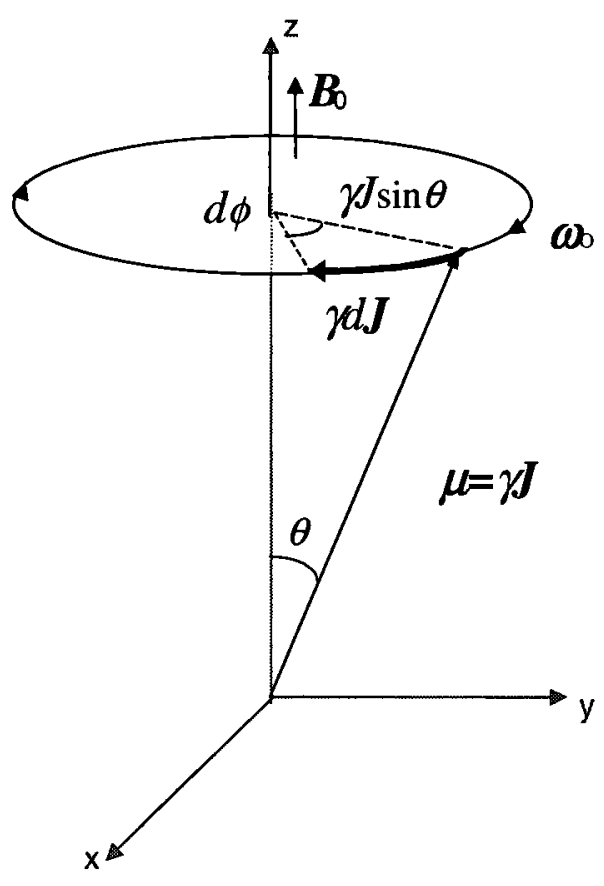

Figure 2.1: Precession of the magnetic moment $\mu$ with angular velocity $\omega_{0}$ about the static magnetic field $\boldsymbol{B}_{0}$.

In a sample comprised of many nuclei, the net magnetic moment $\boldsymbol{M}$ can be defined to be the vector sum of all of the nuclear magnetic moments. Classical theory is inadequate to describe the behaviour of a single spin because of the quantum nature of nuclear properties. However, the behaviour of $\boldsymbol{M}$ obeys the classical laws of physics. In a magnetic field $\boldsymbol{B}_{0}$, the magnetization $\boldsymbol{M}$ at equilibrium will be aligned with $\boldsymbol{B}_{0}$. In order to measure $\boldsymbol{M}$, it must be 
tilted away from $\boldsymbol{B}_{0}$ to produce a component in the $x y$ plane. This is actually the basis of NMR measurements.

The motion of $\boldsymbol{M}$ under the influence of a magnetic field $\boldsymbol{B}_{0}$ can be described by:

$$
\frac{d \boldsymbol{M}}{d t}=\boldsymbol{\mu} \times \mathbf{M} \boldsymbol{B}_{0}
$$

Considering a reference frame rotating with the angular velocity $\omega$ in the $x y$ plane (see Fig. 2.2), the behaviour of $M$ in the laboratory frame can be related to its behaviour in the rotating frame by:

$$
\frac{d M}{d t}=\frac{\partial M}{\partial t}+\omega \times M
$$

where $\frac{\partial \boldsymbol{M}}{\partial t}$ represents the rate of change of $\boldsymbol{M}$ in the rotating frame [87]. Using Eq. (2.6) this can be written as:

$$
\begin{aligned}
\frac{\partial \boldsymbol{M}}{\partial t} & =\gamma \boldsymbol{M} \times \boldsymbol{B}_{0}+\boldsymbol{M} \times \boldsymbol{\omega}=\gamma \boldsymbol{M} \times\left(\boldsymbol{B}_{0}+\frac{\omega}{\gamma}\right) \\
& =\mathcal{M} \times \boldsymbol{B}_{\text {eff }},
\end{aligned}
$$

where $\boldsymbol{B}_{\text {eff }}$ is the sum of the static field $\boldsymbol{B}_{0}$, and a fictitious magnetic field whose direction is the same as $\omega$. The magnetic resonance phenomenon appears when a second magnetic field $\boldsymbol{B}_{1}$ of frequency $\omega=-\omega_{0}=-\not \boldsymbol{B}_{0}$ is applied perpendicular to $\boldsymbol{B}_{0}$.

For magnetic field strengths used in MRI, the resonance frequencies are in the radio frequency range. In the rotating frame, the effective field is:

$$
\boldsymbol{B}_{\text {eff }}=\boldsymbol{B}_{0}+\frac{\omega}{\gamma}+\boldsymbol{B}_{1}
$$


which reduces to $\boldsymbol{B}_{\text {eff }}=\boldsymbol{B}_{1}$, when the resonance condition applies (i.e. $\omega=-\omega_{0}$ ). The corresponding on resonance equation of motion for the magnetization vector $\boldsymbol{M}$ in the rotating frame is:

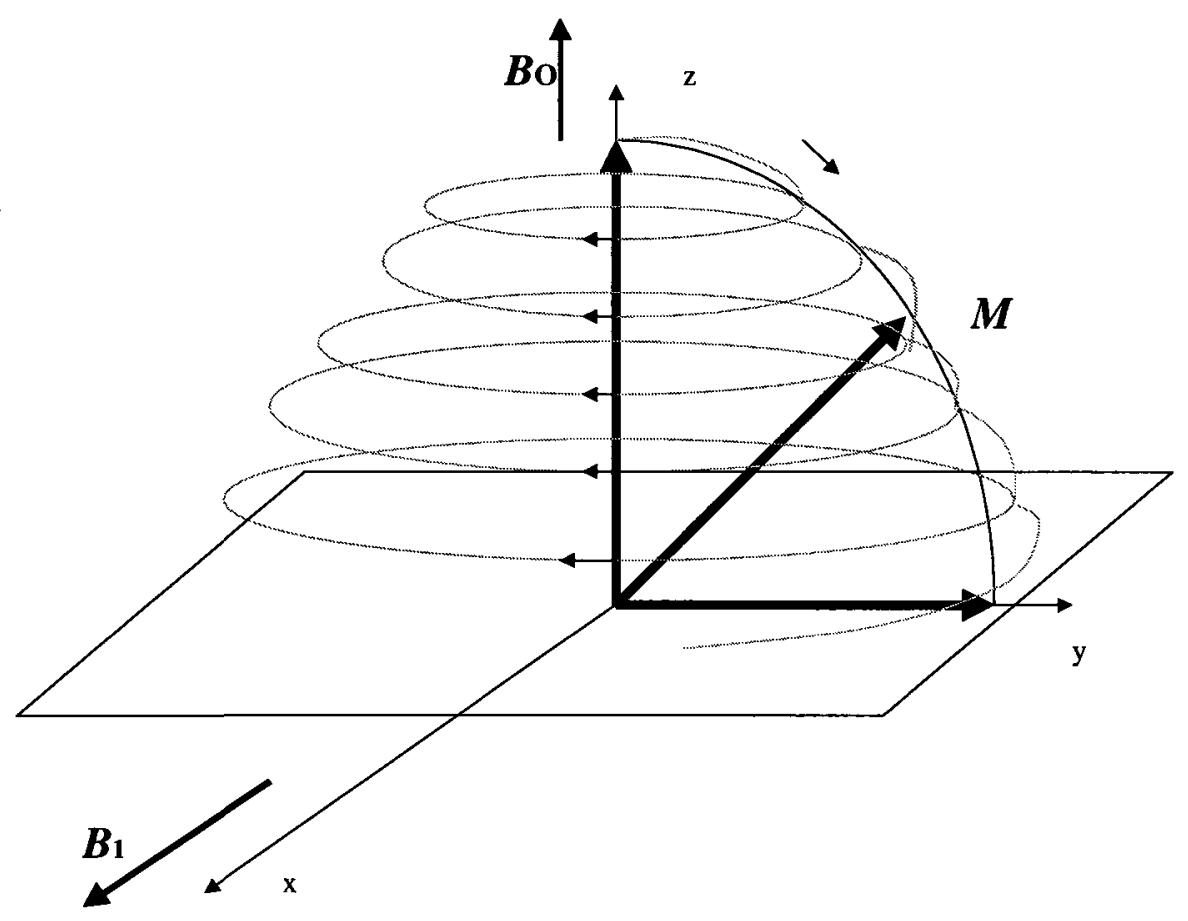

Figure 2.2: The motion of $\boldsymbol{M}$ in the laboratory frame showing the components along the $z$ axis and in the $x y$ plane.

$$
\frac{\partial \boldsymbol{M}}{\partial t}=\boldsymbol{\mu} \boldsymbol{M} \times \boldsymbol{B}_{1} .
$$

The effect of $\boldsymbol{B}_{1}$ is to rotate the magnetization vector $\boldsymbol{M}$ about its direction, with an angular frequency $\omega_{1}=\mathcal{B}_{1}$. If $\boldsymbol{B}_{1}$ is applied on-resonance for a finite time $t$, the magnetization will rotate through an angle:

$$
\theta=\omega_{1} t=\gamma B_{1} t
$$


By varying the amplitude and duration of this radio frequency pulse, any desired angle can be produced.

\subsubsection{Quantum Mechanical Description}

The NMR signal comes from nuclei that have an intrinsic angular momentum $J$. The magnitude of the nuclear angular momentum is given by:

$$
J=\hbar[I(I+1)]^{1 / 2},
$$

where $I$ is the nuclear spin quantum number and $\hbar=\frac{h}{2 \pi}$, with $h$ known as Planck's constant. From the uncertainty principle, the direction of $\boldsymbol{J}$ cannot be determined precisely. By applying an external magnetic field $\boldsymbol{B}_{0}=B_{0} \boldsymbol{k}$, arbitrarily chosen to be aligned along the $z$ direction, an axis of quantization can be defined.

The component of $\boldsymbol{J}$ along the $z$-axis is:

$$
J_{z}=m_{z} \hbar
$$

where $m_{z}$ is called the magnetic quantum number and can take the values: $\pm I, \pm(I-1), \ldots, 0$ giving $(2 I+1)$ possible values [81], each corresponding to a possible orientation for $J$. For the proton, $I=\frac{1}{2}$ and therefore $m_{z}$ can only take the values $\pm \frac{1}{2}$. This gives two possible orientations for the spin, which are referred to as spin up $\left(m_{z}=\frac{1}{2}\right)$ and spin down $\left(m_{z}=-\frac{1}{2}\right)$. 

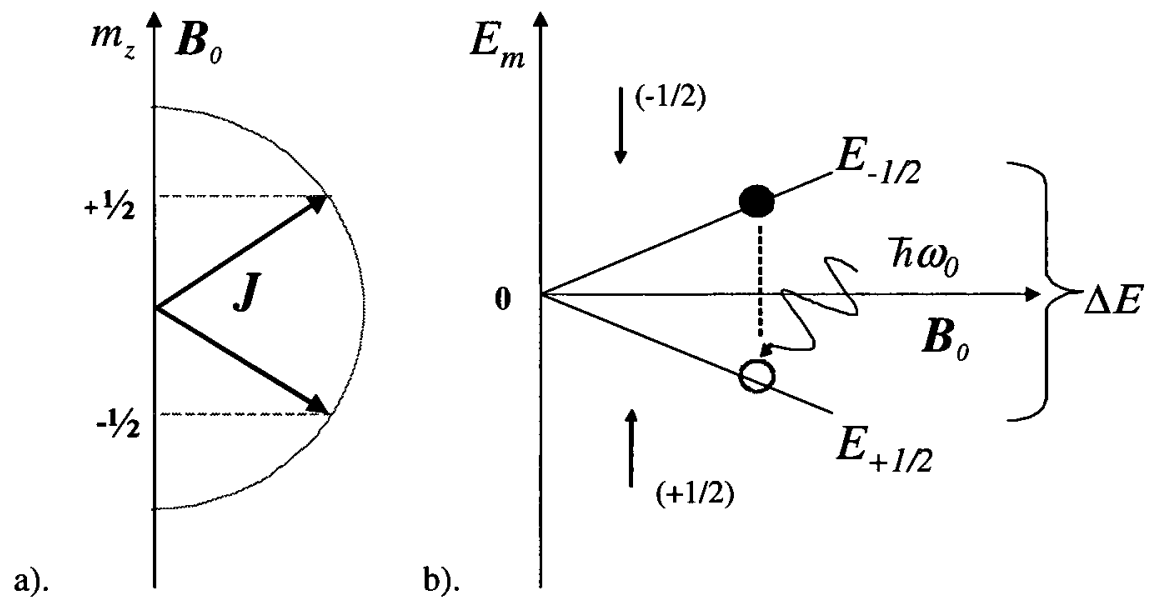

Figure 2.3: a) The available orientations for $J$ and the corresponding values of $m_{z}$, b) Zeeman effect associated with the transition from the lower state to the upper state as a result of absorption of a quantum of energy.

The potential energy of these states is:

$$
E=-\mu B_{0}=-\gamma \hbar J_{z} B_{0}
$$

As shown in Fig. 2.3, the energy levels for a proton are:

$$
E_{+\frac{1}{2}}=-\gamma \frac{\hbar}{2} B_{0} \text { (the lower state energy), }
$$

and

$$
E_{-\frac{1}{2}}=\gamma \frac{\hbar}{2} B_{0} \quad \text { (the upper state energy) }
$$

This is an example of the Zeeman effect, where the nuclear magnetic moment of the spin causes the spin states to split into discrete energy levels in the presence of a magnetic 
field. The energy separation, $\Delta E$, between the two quantum states, gives the magnitude of the energy absorbed or released by the proton spin system following a transition between the lower and higher states. From Eqs. (2.15) and Eq. (2.16) it can be seen that:

$$
\Delta E=\hbar \hbar B_{0}=\hbar \omega_{0},
$$

where $\omega_{0}$ is the Larmor frequency, which was defined in Eq. (2.5) to be $\omega_{0}=\gamma B_{0}$. It takes a specific amount of energy to flip spins between the lower state and upper state.

\subsubsection{Statistical Distribution of Spin States}

For a large sample containing $N_{s}$ spins placed in a magnetic field $\boldsymbol{B}_{0}$, quantum mechanics dictates that the magnetic moment of each individual spin must be aligned in the

direction of the applied field either with spin up $\left(m_{I}=+\frac{1}{2}\right)$, or spin down $\left(m_{I}=-\frac{1}{2}\right)$. The population ratio between the two levels, in equilibrium is described by the Boltzmann distribution:

$$
\frac{n_{\uparrow}}{n_{\downarrow}}=\exp \left(\frac{\Delta E}{k T}\right)=\exp \left(\frac{\not h B_{0}}{k T}\right),
$$

where $k$ is Boltzmann's constant, $T$ is the temperature of the spin system, $n_{\downarrow}$ is the number of spins in the spin down state (i.e. higher energy) and $n \uparrow$ is the number of spins in the spin up state. At room temperature, in a field of $1.5 \mathrm{~T}$, there are, on average, only 10 more protons per million in the lower energy state than in the higher energy state. The difference in population is given by [82]: 


$$
\begin{aligned}
n_{\uparrow}-n_{\downarrow} & =N_{s} \frac{1-\exp \left(\frac{-\Delta E}{k T}\right)}{1+\exp \left(\frac{-\Delta E}{k T}\right)} \\
& =\frac{N_{s}}{2} \frac{\Delta E}{k T}=\frac{N_{s}}{2} \frac{\hbar B_{0}}{k T}
\end{aligned}
$$

where $N_{s}=n_{\uparrow}+n_{\downarrow}$. The net equilibrium magnetization, considering the difference of population between these two levels, can be written as:

$$
M_{0}=\left(n_{\uparrow}-n_{\downarrow}\right) \xi \frac{1}{2}=\frac{N_{s} \gamma^{2} \hbar^{2}}{4 k T} B_{0} .
$$

The intensity of the NMR signal depends on the difference in population between the spin states, which is determined by the magnetic field strength $B_{0}$ and the temperature $T$. The energy difference between the states varies linearly with the applied magnetic field. All the signals generated in MRI are based on the small population difference between the two states, explaining why MR techniques are limited by signal strength.

\subsection{Bloch's Equation and Relaxation}

When RF energy is no longer being supplied to the nuclei, they begin to give-off the absorbed energy and revert back to their equilibrium state. The molecular environment is reflected in the time-variation of the signal amplitude. The release of energy occurs in two ways. Energy is given off by the spin system to the other energy reservoirs in the material being studied and normally manifests itself as heat. This process is known as spin-lattice or longitudinal relaxation and can be characterised by the time constant, $T_{1}$. The other energy transfer process involves the exchange of energy among the nuclear spins, such that the total 
energy of the spin system remains unchanged. This process is referred to as spin-spin or transverse relaxation, and in fluids, can be characterized by the time constant, $T_{2}$. The relaxation process by which the thermal equilibrium is re-established following the application of a RF pulse can be modelled phenomenologically by Bloch's equations.

When the "motion" due to relaxation is superimposed on the precessional motion of the free spins under the influence of a static field and a much smaller RF field, the rate of change of the magnetization is given by [83]:

$$
\frac{d \boldsymbol{M}}{d t}=\boldsymbol{M} \times \boldsymbol{B}-\left(\frac{M_{x}}{T_{2}} \boldsymbol{i}+\frac{M_{y}}{T_{2}} \boldsymbol{j}\right)-\frac{\left(M_{0}-M_{z}\right)}{T_{1}} \boldsymbol{k},
$$

where $\boldsymbol{i}, \boldsymbol{j}, \boldsymbol{k}$ are unit vectors in the laboratory frame. From Eq. (2.21), which is known as Bloch's equation, it can be seen that the longitudinal magnetization displays an exponential form of evolution to its equilibrium value $M_{0}$ given by

$$
M_{z}=M_{0}\left(1-e^{-t / T_{1}}\right) \text {, }
$$

and for the precessing transverse component in the rotating frame:

$$
M_{x y}=M_{0} e^{-i \gamma B_{z} t-t / T_{2}} .
$$

The observed signal in an MR experiment comes from the detection of the emf generated by the precessing transverse magnetization. Once a component of the magnetization is tipped into the $x y$ plane, a free induction decay, FID, signal is generated if a suitable coil is used. This characteristic signal has a frequency of oscillation equal to the Larmor frequency.

In an ideal system with a perfectly uniform magnetic field, the exponential envelope of the FID signal in the xy plane decays according to Eq. (2.23). In reality, field inhomogeneities cause the signal to decay more quickly, masking the true relaxation characteristics of the tissue. This observed relaxation in tissue is called $T_{2}^{*}$. The decay of transverse magnetization 
occurs because of dephasing of the spins as they precess. Several different mechanisms can cause this dephasing. When it is a result of different spins experiencing different magnetic fields due to inhomogeneities in the applied field then the dephasing can be reversed. This can be done by using a $180^{\circ} \mathrm{RF}$ pulse. This in turn causes the transverse magnetization to rephase and form an echo event, which produces a detectable RF signal. The intensity of the echo signal is proportional to the level of transverse magnetization, which is determined by the relaxation rate $T_{2}$.

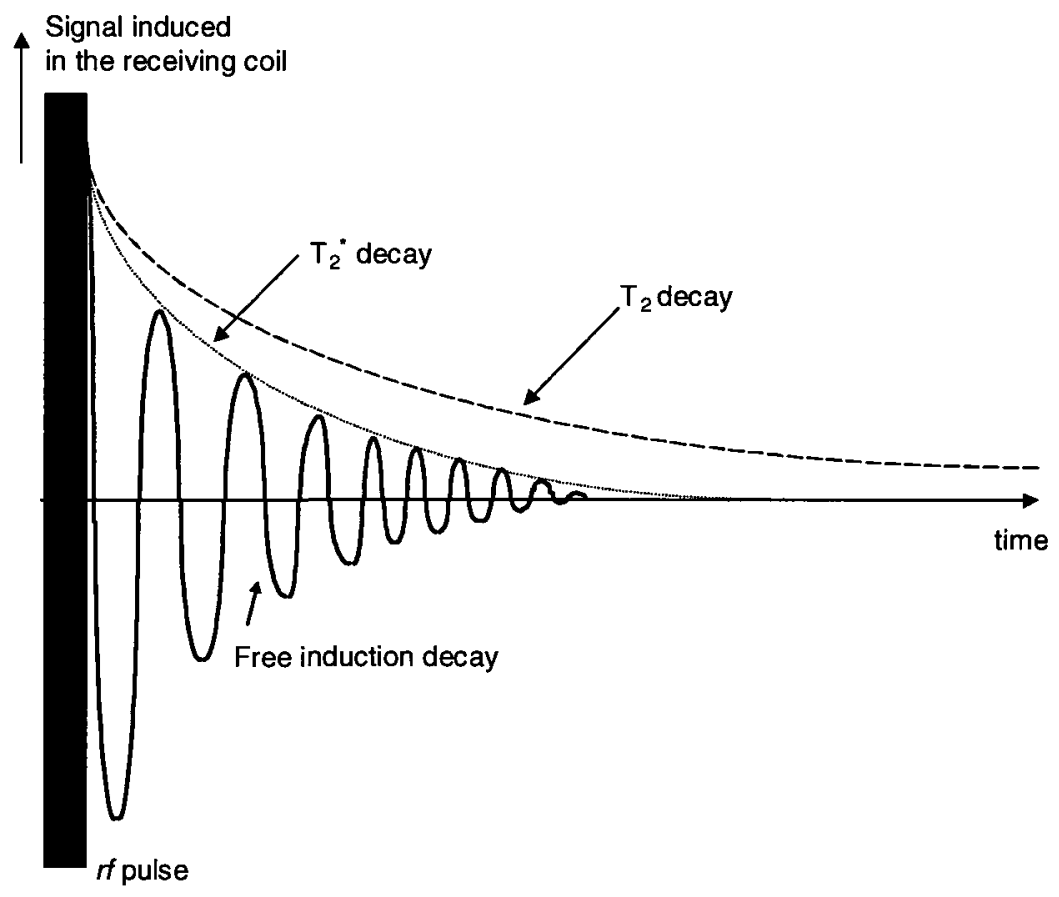

Figure 2.4: The resultant off-resonance FID signal induced by the $x y$ component of the sample magnetization. 


\subsection{Spatial Encoding of the NMR Signal}

During the MR image formation process, the volume that is analyzed is "subdivided" to form a matrix of individual tissue voxels. The RF signal coming from each individual voxel must be distinguished from all of the others and its intensity displayed in the corresponding image field. The result of the image acquisition process is a large amount of data collected by using magnetic field gradients that give in turn to the RF signal their frequency and phase characteristics. The signals from all voxels are produced simultaneously and are emitted mixed together to form a single, composite signal.

While there may be more than one way to analyze a data set, in no case can the final image be any better than the information which was coded into it. In addition to encoding spatial information into a data set, each MRI method must have an accompanying decoding strategy which unravels the coded information to produce spatially resolved images. This process, called the reconstruction process, converts the collected data into an actual image. The signals are sorted out using Fourier transform methods and delivered to the correct pixel address.

\subsection{Diffusion}

This section describes approaches for dealing with translational motion in molecular ensembles in a fluid state. The properties of the diffusion tensor for characterizing water diffusion in biological tissues are also discussed. 


\subsubsection{Diffusion Theory}

In general, the random thermal motion of molecules can be characterized by a timedependent displacement, $\boldsymbol{r}(t)$, due to Brownian motion. This function is considered to vary in a random way from molecule to molecule. In equilibrium, the number density of particles with a given position $r$ at time $t$ denoted $n(r, t)$ is independent of position. In the case of an initially non-uniform molecular distribution, the system will tend to increase its entropy by a net motion of the molecules to make the concentration $n(r, t)$ more uniform.

The classical description of diffusion is via Fick's law where particle flux $J(r, t)$ is considered to be proportional to the particle concentration gradient [82].

$$
\boldsymbol{J}(\boldsymbol{r}, t)=-D \nabla n(\boldsymbol{r}, t),
$$

where $D$ is defined to be the diffusion coefficient. Because there is no mass motion of the system as a whole, the continuity theorem applies [82] and:

$$
\nabla J(\boldsymbol{r}, t)+\frac{\partial n(\boldsymbol{r}, t)}{\partial t}=0
$$

Combining Eqs. (2.24) and (2.25) the diffusion equation known as Fick's second law can be obtained:

$$
\nabla^{2} n(\boldsymbol{r}, t)-\frac{1}{D} \frac{\partial n(\boldsymbol{r}, t)}{\partial t}=0
$$

For a given distribution of $N$ molecules the initial condition

$$
n(\boldsymbol{r}, 0)=\delta\left(\boldsymbol{r}-\boldsymbol{r}_{0}\right)
$$

applies.

The solution to Eq. (2.26), with the appropriate boundary condition $n(r, t) \rightarrow 0$ as $r \rightarrow \infty$, satisfying the initial condition of Eq. (2.27), follows a Gaussian distribution [84]: 


$$
n(r, t)=\frac{N_{s}}{(4 \pi D t)^{3 / 2}} \exp \left[\frac{-\left(\boldsymbol{r}-\boldsymbol{r}_{0}\right)^{2}}{4 D t}\right]
$$

where, from the conservation theory of a fluid:

$$
\int_{0}^{\infty} n(r, t) 4 \pi r^{2} d r=N_{s}
$$

As can be seen from Eq. (2.28), $n(\boldsymbol{r}, t)$ depends only on the net displacement $\left(\boldsymbol{r}-\boldsymbol{r}_{0}\right)$. The average squared displacement, (i.e. the variance), which gives a measure of how the distribution is spread about the mean value, $r_{0}$, can be determined based on the probability of moving from position $\boldsymbol{r}$ to $\boldsymbol{r}_{0}$ during diffusion time $t_{d}$. The resulting expression is:

$$
\left\langle\left(\boldsymbol{r}-\boldsymbol{r}_{0}\right)^{2}\right\rangle=\frac{4 \pi}{N_{s}} \int_{0}^{\infty} n\left(\boldsymbol{r}, t_{d}\right)\left(\boldsymbol{r}-\boldsymbol{r}_{0}\right)^{2} d r=2 d D t_{d},
$$

where $d=1,2$ or 3 representing the dimensionality of the system. Eq. (2.30) is known as Einstein's equation.

The self-diffusion coefficient of pure water can also be shown to be given by [4]:

$$
D=\frac{R T}{N_{A} f}=\frac{k T}{f},
$$

where $R$ is the universal gas constant, $T$ is the absolute temperature, $N_{A}$ is Avogadro's number and $f$ is the friction factor which can be expressed as a function of water viscosity and size of the water molecules. The value of the diffusion coefficients of pure water is $2.3 \times 10^{-3} \mathrm{~mm}^{2} / \mathrm{s}$ at $25^{\circ} \mathrm{C}$ and $3.07 \times 10^{-3} \mathrm{~mm}^{2} / \mathrm{s}$ at $37^{\circ} \mathrm{C}$ [5]. 


\subsubsection{Apparent Diffusion in Biological Tissues}

Biological tissues differ considerably from the infinite, homogeneous media considered so far. Diffusion in tissue is restricted when boundaries in the medium prevent molecules from moving freely across them. In this case, the diffusion coefficient is no longer a constant of time, because the translational motion of molecules is influenced or restricted by different biological structures. In brain tissue, the diffusion coefficients reported so far are 2 to 10 times smaller than $D$, the self-diffusion coefficient for pure water. Also, the microscopic displacements of the water molecules may be enhanced by other transport phenomena such as blood motion or cerebrospinal fluid, CSF, pulsations, etc [85]. Consequently, the parameter used to characterize the mean squared displacement in tissue is normally referred to as an apparent diffusion coefficient $A D C$ which is defined by [84]:

$$
A D C=\frac{\left\langle\left(\boldsymbol{r}-\boldsymbol{r}_{\boldsymbol{0}}\right)^{2}\right\rangle}{2 d t_{d}} .
$$

When measurement times are very short, most molecules do not have enough time to reach the boundaries so that they behave as though they were diffusing freely. As the diffusion time increases, however, a large fraction of molecules will strike the boundaries, and their displacement distribution will deviate from the behaviour of unrestricted diffusion. The effects of restriction will be reflected as decay in the MR signal when mean diffusion distances are comparable in size with the dimensions of the restricted compartments. As described above, the diffusion process is a three-dimensional process but the molecular mobility may not be the same in all directions; diffusion may be more restricted in one direction than in another. This anisotropy effect is a consequence of the geometrical arrangement of the medium and the 
physical presence of obstacles that obstruct the water molecules. For example, the observed anisotropic water diffusion in white matter is no doubt related to the well-organized arrangement of the myelinated axonal tracts in the white matter tissue which can be visualized, somewhat simplistically, as a set of long parallel cylindrical tubes.

\subsubsection{Experimental Methods for Diffusion MR}

Several techniques exist for the experimental determination of $A D C$ in liquids and gases using radioactive and fluorescent tracers, but these cannot be used for in vivo studies in humans. NMR is well suited for studying the diffusion of water molecules in human tissues in vivo. In principle, it is possible to give a spatial label to nuclei at one time and then to monitor their movement using magnetic field gradients. What is then measured, in effect, is the absolute phase of the spins, which is related to their displacement.

The pulsed-gradient-spin-echo sequence, PGSE, is the most commonly used MR sequence for measuring diffusion [86] and is appropriate not only for studying molecular diffusion but can also provide structural information when diffusion is restricted on the NMR time-scale.

The PGSE experiment consists, in its simplest form, of two identical magnetic fieldgradient pulses of magnitude $G$, duration $\delta$ and separation $\Delta$, inserted into an ordinary NMR spin-echo experiment on either side of the $180^{\circ}$ pulse. This sequence is represented schematically in Fig. 2.5. The molecules are "phase" tagged by the effect of the first gradient pulse and their displacement is determined by the second pulse. 


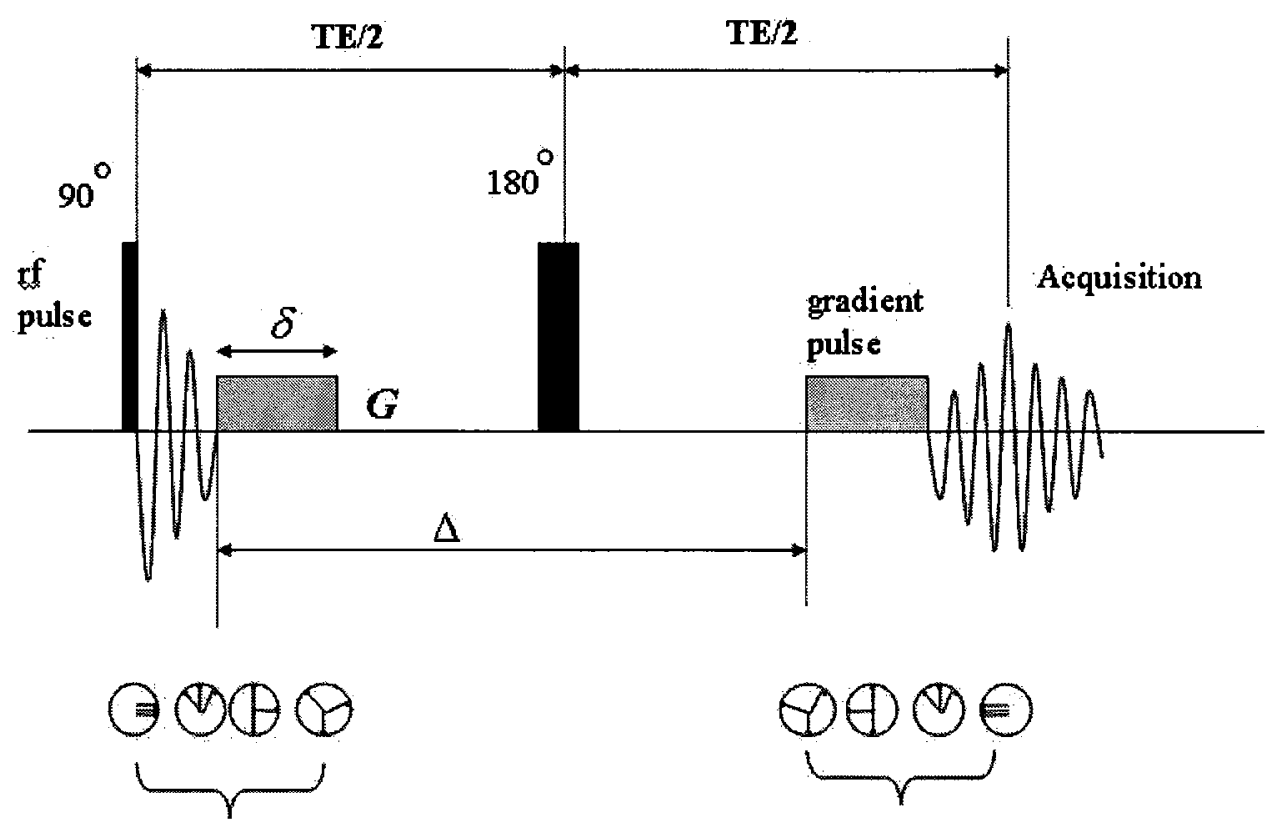

Dephasing Renhasing

Figure 2.5: The PGSE diffusion sequence. After the $90^{\circ}$ pulse, the first diffusion gradient tags the spin by dephasing. The application of the same diffusion gradient after the $180^{\circ}$ pulse partially rephases the spins. Depending on the net displacement during $\Delta$, the incomplete rephasing will cause attenuation of the signal.

The effect of the first gradient is to introduce a phase-shift, $\varphi_{1}$, as a function of the position of the spin, $z_{1}$ :

$$
\varphi_{1}=\int_{0}^{\delta} \omega d t=\gamma \int_{0}^{\delta} G z d t=\gamma G \delta z_{1} .
$$

It is assumed that the gradient pulse duration is short enough that any motion of the spins during the gradient pulse can be ignored. This phase-shift, $\varphi_{1}$ is subsequently inverted by the $180^{\circ}$ pulse such that $\varphi_{1}$ becomes $-\varphi_{1}$. 
The second gradient is then applied to rephase the spins, by introducing an additional phase shift, $\varphi_{2}$, which is proportional to the position of the spin, $z_{2}$, along $z$-axis:

$$
\varphi_{2}=\gamma \int_{\Delta}^{\delta+\Delta} G z d t=\gamma G \delta z_{2}
$$

The net dephasing is given by:

$$
\varphi=\varphi_{2}-\varphi_{1}=\gamma G \delta\left(z_{2}-z_{1}\right)
$$

If the spins are stationary, a perfectly refocused $T_{2}$ modulated echo will occur (i.e. $\varphi$ $=0$ ). But any motion of the spins in the direction of the magnetic field gradient will cause phase shifts in their contribution to the echo leading to an additional attenuation of the measured signal which is proportional to the particle displacement. To remove the effects due to $T_{2}$ relaxation, the signal $M$ is normalized with respect to $M_{0}$, the signal measured in the absence of the diffusion gradients $G$. The time that the spins are allowed to diffuse between labelling and detection, the diffusion time, has been shown by Tanner [87] to be given by $t_{d}=\Delta-\delta / 3$ in the limit $\delta<<$.

The total signal is a superposition of transverse magnetizations in which each phase term is weighted by the probability $P\left(z_{2} \mid z_{1}, \Delta\right)$ for a spin to begin at $z_{1}$ and to move to $z_{2}$ during a period $\Delta$. For a homogeneous system:

$$
M=M_{0} \sum_{j} \exp \left(i \varphi_{j}\right)
$$

For spins diffusing within a free homogeneous system, the attenuation of the transverse magnetization is given by [84]: 


$$
M / M_{0}=\int_{-\infty}^{\infty} \int_{-\infty}^{\infty} \exp \left[i \gamma G \delta\left(z_{1}-z_{2}\right)\right] \rho\left(z_{1}\right) P\left(z_{2} \mid z_{1}, \Delta\right) d z_{1} d z_{2}
$$

where $\rho\left(z_{1}\right)$ represents the initial density of spins. But since $\rho\left(z_{1}\right)$ is constant, Eq. (2.37) reduces to:

$$
M / M_{0}=\int_{-\infty}^{\infty} \exp \left[i \gamma G \delta\left(z_{1}-z_{2}\right)\right] P\left(z_{2} \mid z_{1}, \Delta\right) d z_{2}
$$

For free diffusion in a fluid, $P\left(z_{2} \mid z_{1}, \Delta\right)$ can be equated with $\frac{n(r, t)}{N_{s}}$. This gives, according to Eq. (2.28), the following:

$$
P\left(z_{2} \mid z_{1}, \Delta\right)=(4 \pi D t)^{-1 / 2} \exp \left[\frac{-\left(z_{2}-z_{1}\right)^{2}}{4 D t}\right] \text {. }
$$

Substituting this into Eq. (2.38) gives:

$$
\begin{aligned}
M / M_{0} & =(4 \pi D t)^{-1 / 2} \int_{-\infty}^{\infty} \exp \left[i \gamma G \delta\left(z_{1}-z_{2}\right)\right] \exp \left[\frac{-\left(z_{2}-z_{1}\right)^{2}}{4 D t}\right] d z_{2} \\
& =(4 \pi D t)^{-1 / 2} \int_{-\infty}^{\infty} \cos \left[\gamma G \delta\left(z_{1}-z_{2}\right)\right] \exp \left[\frac{-\left(z_{2}-z_{1}\right)^{2}}{4 D t}\right] d z_{2} \\
& \left.=\exp \mid-(\gamma G \delta)^{2} D \Delta\right] .
\end{aligned}
$$

Using Eq. (2.30), this equation becomes:

$$
M(T E, G) / M_{0}(T E, G=0)=\exp \left[\frac{-(\gamma G \delta)^{2}\left\langle\Delta z^{2}\right\rangle}{2}\right] .
$$

Eq. (2.41) constitutes the key for all MR diffusion measurements. However, there are also two comments to make regarding this expression. First, although it is frequently assumed that $\delta<\Delta, \delta$ is not always negligible with respect to $\Delta$, so that diffusion during the gradient 
pulses cannot always be ignored. Second, the imaging gradients will also affect the spin dephasing and rephrasing but their overall effect on the diffusion decay is normally quite small compared with the effect of the diffusion gradients. These considerations are not considered in the derivation of Eq. (2.41).

In NMR experiments, the diffusive transport of magnetization is accounted for by modifying the Bloch equation to include a term that describes the diffusion [88]:

$$
\frac{d \boldsymbol{M}}{d t}=\boldsymbol{M} \times \boldsymbol{B}-\left(\frac{M_{x}}{T_{2}} \boldsymbol{x}+\frac{M_{y}}{T_{2}} \boldsymbol{y}\right)+\frac{\left(M_{0}-M_{z}\right)}{T_{1}} z+D \nabla \cdot(\nabla \boldsymbol{M}) .
$$

In the case of a PGSE experiment, the spins experience the main static field $\boldsymbol{B}_{0}$ superimposed with the linear gradient $\boldsymbol{G}$, so that

$$
\boldsymbol{B}(\boldsymbol{r}, t)=\boldsymbol{B}_{0}+\boldsymbol{G} \boldsymbol{r}
$$

where $\boldsymbol{r}$ represents the position vector. Also, the transverse magnetization $M_{x y}(\boldsymbol{r}, t)$, can be written as:

$$
M_{x y}(r, t)=M_{x}(r, t)+i M_{y}(r, t) .
$$

By combining Eqs. (2.43) and (2.44) and ignoring the relaxation terms, the equation of motion for $M_{x y}$ in the rotating frame becomes:

$$
\frac{d M_{x y}(\boldsymbol{r}, t)}{d t}=-i \gamma \boldsymbol{G} \cdot \boldsymbol{r}+D \nabla \cdot\left(\nabla M_{x y}(\boldsymbol{r}, t)\right)
$$

This equation has the following solution when $D$ is set to zero:

$$
M_{x y}(\boldsymbol{r}, t)=A \exp \left(-i \gamma \cdot \int_{0}^{t} \boldsymbol{G}\left(t^{\prime}\right) d t\right)
$$

The exponential term gives the phase shift of a spin at position $r$ checked at time $t$. For $D \neq 0$, a trial solution can be generated by setting $A \rightarrow M_{0} A(t)$ in Eq. (2.46) for spatially 
invariant diffusion. Substitution of this trial solution back into Eq. (2.45), after considerable simplification, gives:

$$
\begin{gathered}
M_{x y}(t)=M_{0} \exp \left[-D \gamma^{2} \int_{0}^{T E}\left(\int_{0}^{t} G\left(t^{\prime \prime}\right) d t^{\prime \prime}\right)^{2} d t^{\prime}\right] \\
=M_{0} \exp [-b D] .
\end{gathered}
$$

Here the gradient factor $b$, which is often called the $b$-value, was defined as [89]:

$$
b=\gamma^{2} \int_{0}^{T E}\left(\int_{0}^{i} G\left(t^{\prime \prime}\right) d t^{\prime \prime}\right)^{2} d t^{\prime} .
$$

Eq. (2.46) represents the magnetization attenuation due to the diffusion of spins. The diffusion integral of the $b$-value can be solved numerically for various NMR sequences and diffusion gradient pulse profiles. The $b$-value gives a measure of the gradient strength in the diffusion sequence.

\subsection{The Physics of $\mathrm{T}_{2}$ Relaxation Mechanism}

In order to understand what $T_{2}$ represents, it is necessary to understand NMR relaxation theory. $T_{2}$ relaxation causes the decay of the transverse component of the bulk magnetization, $\boldsymbol{M}_{\mathrm{xy}}$ and the rate at which the signal for a pure homogeneous liquid dies away is described by Eq. (2.23). Of particular note, for many spin $1 / 2$ nuclei, this relaxation mechanism derives solely from the interaction of the field of a tumbling nuclear dipole with other dipole fields; the so called 'dipole-dipole' interaction. The concept of $T_{2}$ cannot be understood as a property of a single nucleus but rather it is a property of an ensemble of similar nuclei. 


\subsubsection{Basics of the Dipole-Dipole Interaction}

In classical physics, the interaction energy $E$ between two magnetic moments, $\boldsymbol{\mu}_{1}$ to $\boldsymbol{\mu}_{2}$, is defined, in SI units, as [89]:

$$
E=\frac{\mu_{0}}{4 \pi}\left[\frac{\mu_{1} \cdot \mu_{2}}{r^{3}}-\frac{3\left(\mu_{1} \cdot r\right)\left(\mu_{2} \cdot r\right)}{r^{5}}\right]
$$

where $\boldsymbol{r}$ is the distance vector from $\boldsymbol{\mu}_{1}$ to $\boldsymbol{\mu}_{2}$ and $\mu_{0}$ is the permeability of free space. The general contribution to the Hamiltonian of an $N$ spin system is given by:

$$
H_{D}=\frac{\mu_{0}}{8 \pi} \sum_{j=1}^{N} \sum_{k=1}^{N} \frac{\boldsymbol{\mu}_{j} \cdot \boldsymbol{\mu}_{k}}{r_{j k}^{3}}-\frac{3\left(\boldsymbol{\mu}_{j} \cdot \boldsymbol{r}_{j k}\right)\left(\boldsymbol{\mu}_{k} \cdot \boldsymbol{r}_{j k}\right)}{r_{j k}^{5}}
$$

By writing $\mu_{1}$ to $\mu_{2}$ in terms of $I_{1}$ and $I_{2}$, using Eq. (2.1), and the $x$ and $y$-components in terms of the raising and lowering operators defined by:

$$
I^{ \pm}=I_{x} \pm i I_{y}
$$

the $\mu_{j}$ can be written as:

$$
\mu_{j}=\frac{\not \hbar}{2}\left[\left(I_{j}^{+}+I_{j}^{-}\right) \hat{\boldsymbol{x}}-i\left(I_{j}^{+}-I_{j}^{-}\right) \hat{\boldsymbol{y}}+2 I_{j z} \hat{z}\right],
$$

where it has been assumed that $\mu_{1}$ to $\mu_{2}$ are of the same nuclear species and $i=\sqrt{-1}$. By inserting this expression into Eq. (2.50) with considerable simplification, one can write [89]:

$$
H_{D}=\frac{\mu_{0} \gamma^{2} \hbar^{2}}{4 \pi r^{3}}(A+B+C+D+E+F),
$$

where

$$
\begin{gathered}
A=I_{1 z} I_{2 z}\left(1-3 \cos ^{2} \theta\right), \\
B=-\frac{1}{4}\left(I_{1}^{+} I_{2}^{-}+I_{1}^{-} I_{2}^{+}\right)\left(1-3 \cos ^{2} \theta\right),
\end{gathered}
$$




$$
\begin{gathered}
C=-\frac{3}{2}\left(I_{1}^{+} I_{2 z}+I_{1 z} I_{2}^{+}\right)\left(\sin \theta \cos \theta e^{-i \phi}\right), \\
D=-\frac{3}{2}\left(I_{1}^{-} I_{2 z}+I_{1 z} I_{2}^{-}\right)\left(\sin \theta \cos \theta e^{i \phi}\right), \\
E=-\frac{3}{4}\left(I_{1}^{+} I_{2}^{+} \sin ^{2} \theta e^{-2 i \phi}\right), \\
F=-\frac{3}{4}\left(I_{1}^{-} I_{2}^{-} \sin ^{2} \theta e^{2 i \phi}\right) .
\end{gathered}
$$

and $\theta$ is the angle between $r$ and $\hat{z}$ and $\phi$ is the azimuthal angle.

The $A$ and $B$ terms may be combined to obtain what is called the secular term [89]:

$$
H_{D}^{0}=\frac{\mu_{0} \gamma^{2} \hbar^{2}}{8 \pi r^{3}}\left(1-3 \cos ^{2} \theta\right)\left(3 I_{1 z} I_{2 z}-I_{1} \cdot I_{2}\right)
$$

The complicated dipole-dipole Hamiltonian has enormous significance for the nuclear spin system and its magnitude, $E_{D}$, is

$$
E_{D} \sim \frac{\mu_{0}}{4 \pi} \frac{\gamma^{2} \hbar}{r^{3}}
$$

The dipolar interaction between spins is weak in comparison with the strong Zeeman interaction of the spins with the applied magnetic field $B_{\mathrm{o}}$. The secular terms give shifts in the energy levels on the scale of $E_{D}$. The remaining terms $C, D, E$ and $F$, the non-secular terms, produce only second order shifts in the energy levels but they play a very important role in both $T_{1}$ and $T_{2}$ relaxation.

In liquid samples very sharp water NMR spectral lines are observed because of molecular tumbling which causes the time-averaged $H_{D}$ to be effectively zero. Conversely, due to restricted motion in more rigid states (e.g. bound states), the fluctuating fields and dipolar interactions are not totally averaged such that, their residual effects on the NMR spectra 
constitute a valuable source of information concerning the mechanisms of relaxation rates and molecular interactions. These effects manifest themselves as a broadening of the resonance line, the width of which is proportional to $1 / T_{2}$. Moreover, at low temperatures, the averaging of dipolar and quadrupolar interactions is less effective because of reduced thermal motion, orientation, and ordering of water molecules. In intermediate states, such as gels, the motional averaging is not complete and, although the effectiveness of the dipolar coupling is reduced from its full value, it is not completely averaged to zero. Often the motions of the dipoles will be anisotropic with different rotational rates about different axes leading to anisotropy of the effective dipole-dipole interactions. This can be a very strong effect in solids but exists to a lesser extend in other states such as gels.

\subsubsection{Relaxation and Molecular Motion}

Spin-lattice and spin-spin relaxation of protons are induced by fluctuations in the local magnetic field around a nucleus due to interactions between spins. The relaxation times are sensitive, not only to the magnitude of these fluctuations but also to their rates. Nuclear magnetic relaxation is partially characterized by molecular mobility and intermolecular interactions. The effects of molecular motion are generally incorporated into the theory of nuclear magnetic relaxation in terms of the correlation time, $\tau_{c}$, which is considered to be the time taken by a molecule to translate through a molecular distance, or the average time between molecular collisions for a molecule in its actual state of motion [60]. For rotational motions it is considered to be the average time it takes to rotate one radian [90]. The molecules tumble at various rates and in various directions; they collide and change orientation over a range of rates up to a maximum rate of approximately $\left(1 / \tau_{\mathrm{c}}\right)$. A quantity of prime interest in 
relaxation theory relates to the concentration or intensity of these fluctuating local magnetic fields at a given angular frequency, $\omega$. This function is known as the spectral density function, $J(\omega)$, where [89]

$$
J(\omega)=\frac{\tau_{c}}{1+\left(\omega \tau_{c}\right)^{2}}
$$

The intensity of the spectral density function at the Larmor frequency is of particular interest since these fluctuating magnetic fields induce transitions among the Zeeman energy levels that lead to $T_{1}$ and $T_{2}$ relaxation.

In liquid water, with no paramagnetic impurities, the predominant effect is a modulation of intra-molecular dipolar interactions by Brownian rotation of water molecules [60]. In this case, $\tau_{\mathrm{c}}$ is approximately the average time for a molecule to rotate through one radian. The $T_{2}$ relaxation time for two nuclear spins can then be evaluated using the spectral density approach and is given by [91]:

$$
\frac{1}{T_{2}}=\left(\frac{\mu_{0}}{4 \pi}\right)^{2} \frac{\gamma^{4} \hbar^{2} I(I+1)}{4 r^{6}}\left[\frac{3}{2} J(0)+15 J\left(\omega_{0}\right)+\frac{3}{2} J\left(2 \omega_{0}\right)\right]
$$

where it was assumed that $\gamma_{1}=\gamma_{2}=\gamma$. For a water molecule undergoing isotropic rotations, and considering only the intramolecular interaction, this becomes [91]

$$
\frac{1}{T_{2}}=\left(\frac{\mu_{0}}{4 \pi}\right)^{2} \frac{\gamma^{4} \hbar^{2} I(I+1)}{5 r^{6}}\left[3 \tau_{c}+5 J\left(\omega_{0}\right)+2 J\left(2 \omega_{0}\right)\right]
$$

where $r$ is the distance between the protons in a water molecule. It becomes obvious that the dipolar couplings are significantly more active for nuclei located in the same molecule due to, $r$, the internuclear distance.

In free or bulk water, the rotational and translational motions of water molecules are strongly coupled; as a result the motion of water molecules can be defined by a single 
correlation time [92]. Moreover, the molecular tumbling changes the orientation of the spin axis on a time scale which is faster than the dipole-dipole couplings. The rotational correlation times are on the order of picoseconds to nanoseconds [93]. This corresponds to reorientation rates of $10^{7}-10^{11}$ radians/s as compared to the dipolar couplings which are on the order of $10^{5}$ radians/s [90]. Therefore, in a pure liquid, the secular part of the dipole-dipole coupling corresponds to an average of $\left(3 \cos ^{2} \theta-1\right) B_{0}$ over the isotropic distribution of orientations.

For biological water the situation is dramatically different, since, in the hydration layers, the translational and rotational motions are no longer appreciably coupled due to molecular interactions, restricted motion and ordering of water molecules. Under the influence of the decoupling effect, the motion of the entire mass of water cannot be expressed by a single correlation time but requires a distribution function. Some NMR studies that investigated water associated with macromolecular systems have indicated that the water is indeed disturbed due to the presence of the proteins and that the reorientation correlation time is clearly larger than the nanosecond scale [94]. Typical figures for correlation times for different water states are: for super bound water, $\tau_{\mathrm{c}} \approx 10^{-6} \mathrm{~s}$, for polar-bound water, $\tau_{\mathrm{c}} \approx 10^{-9} \mathrm{~s}$ and for structured water $\tau_{\mathrm{c}} \approx 10^{-11} \mathrm{~s}[95,96]$. Some confirmation that, the correlation times obtained for the "bound" water phases are of the same order as protein rotational relaxation times measured in dielectric dispersion experiments was obtained by Oncley [97]. However, the presented results are strongly dependent on water content and temperature.

The detailed explanation of relaxation phenomena in macromolecular solutions is still a matter of debate; however, most of the experimental predictions are straightforward and can be interpreted in terms of an overall reduction in molecular mobility. Water found in a physical state which differs from bulk water and which has a relatively fixed orientation or position, (i. 
e. bound water or structured water), will have more efficient spin-spin interactions and will experience a heterogeneous local field that causes selective $T_{2}$ relaxation.

\subsubsection{NMR Studies of Water in Biological Systems}

The study of nuclear spin relaxation phenomena by pulsed NMR techniques can, in principle, provide information on both structural and binding effects as defined above. Changes in water structure might be expected to be reflected in changes in those relaxation times which are determined by nuclear magnetic dipole-dipole interactions. The early papers by Cope [98] and Hazlewood et al [99] proposed that there exist no less than two phases of ordered water in many biological tissues including skeletal muscle. Independent confirmation of the existence of two categories of water in frog nerve was obtained from NMR experiments by Swift and Fritz in 1967 [100]. Since then, the classical view of cell water has been continuously challenged by evidence coming from NMR spectra and relaxation times. For example, approximately $75 \%$ of water protons in erythrocytes have an NMR spectrum that is broadened fourfold [101]. The NMR spectrum of water in fish muscle shows moderate broadening [102]. The NMR spectrum of water in nerve shows a partial splitting into two peaks [67], or slight broadening [100]. NMR relaxation times of protons in muscle water were shown to be much shorter than in liquid water and to be influenced by contraction of the muscle [103]. Unfortunately, the interpretation of these data is complicated by the fact that broadening of proton NMR spectra may also be produced by paramagnetic ions or by microscopic magnetic inhomogeneities.

Independently of the above studies, $T_{2}$ relaxation MR measurements have long been exploited for studying the organization and distribution of water in living tissues. Early studies 
performed with non-imaging based Carr-Purcell-Meiboom-Gill (CPMG) sequences [104] or Hahn spin echo sequences [105] on tissue specimens often described the $T_{2}$ relaxation decay curves as being non-monoexponential in nature and well-suited to multiexponential analyses, with the components commonly interpreted in terms of different water compartments with some contributions from macromolecules, particularly for the very short $T_{2}$ components [106109].

\subsection{Diffusion Tensor Imaging}

Diffusion Weighted MRI (DWI) is a relatively new, non-invasive imaging technique, based on traditional MRI, which is highly sensitive to the random motion of the water molecules in the voxel. Although water appears to be calm to the naked eye, water molecules within tissues are constantly in motion. This phenomenon is commonly referred to as "Brownian motion". It is well known that in tissue, the presence of various tissue components, such as cell membranes and large protein molecules, dampens this Brownian motion. When these obstructions have a well defined geometry the resulting diffusion can be anisotropic. In this case, the diffusion cannot be characterized using a single diffusion coefficient and it is necessary to use a diffusion tensor instead. Diffusion Tensor MRI (DTI) provides such complex information about how water diffuses in different directions in tissues making this technique ideally suited to quantitatively characterize local structure in tissues such as bone, muscle and brain white matter [110-112].

Anisotropic diffusion is best characterized by a symmetric, positive, second rank tensor

$\bar{D}$. This mathematical construct, which is used here to represent an intrinsic property of the 
tissue, reflects the diffusion of water along specific directions in the laboratory frame of reference:

$$
\overline{\boldsymbol{D}}=\left(\begin{array}{lll}
D_{x x} & D_{x y} & D_{x z} \\
D_{y x} & D_{y y} & D_{y z} \\
D_{z x} & D_{z y} & D_{z z}
\end{array}\right)
$$

The diffusion tensor $\bar{D}$ can be decomposed into its eigenvalues and eigenvectors. These words are derived from the German word "eigen" which means "characteristic". If there is a nonzero vector $\mathbf{v}$ such that:

$$
\bar{D}_{\mathbf{v}}=\lambda \mathbf{v}
$$

then, by definition, $\lambda$ is an eigenvalue of $\bar{D}$ and $\mathbf{v}$ is the corresponding eigenvector. The solutions $\lambda_{i}$ to Eq. (2.67) can be evaluated by solving the following determinant:

$$
\operatorname{det}(\overline{\boldsymbol{D}}-\lambda \mathbf{I})=0
$$

where $\mathbf{I}$ is the second rank identity matrix. This equation is called the characteristic equation of $\bar{D}$ and the eigenvalues of $\overline{\boldsymbol{D}}$ are all solutions of this equation. For a real symmetric tensor, like the diffusion tensor, the eigenvalues are always real and the eigenvectors are orthogonal on each other. Using this property, the diffusion tensor can be diagonalized such that only the three non-zero eigenvalues $\lambda_{1}, \lambda_{2}$ and $\lambda_{3}$ remain along the diagonal:

$$
\overline{\boldsymbol{D}}=\left(\begin{array}{lll}
\lambda_{1} & 0 & 0 \\
0 & \lambda_{2} & 0 \\
0 & 0 & \lambda_{3}
\end{array}\right)
$$

The trace of the diffusion tensor is invariant to rotations and is defined as [53]:

$$
\operatorname{trace}(\bar{D})=\sum_{i} D_{i i}=\sum_{n} \lambda_{n}
$$


To investigate axonal structures, the diffusion tensor for each pixel has to be fully characterized based on its eigenvalues and their corresponding eigenvectors. From this characterization, two important parameters can be obtained: the axonal direction and the fractional anisotropy. Assuming that water molecules in white matter tends to diffuse more easily along the axonal fibers, the orientation of the axons can be determined by identifying the direction of the eigenvector corresponding to the largest eigenvalue as being the direction of the main fiber bundle in the voxel. Once the predominant fiber direction for each voxel is known, it is possible to reconstruct three-dimensional (3D) fiber structures by connecting the corresponding pixels through multiple image slices. This technique of 3D-diffusion MRI reconstruction, known as tractography [110], is by far the most important non-invasive tool used to observe human white matter tracts in vivo and to study the connectivity of functional centers of the brain, brain development, and white matter diseases.

An important aspect of DTI measurements is the influence on the measured contrast from the tissue microstructure. Images with true microscopic resolution cannot be constructed from DTI measurements since, in a typical DTI measurement, the voxel dimensions are of the order of 1 to $5 \mathrm{~mm} /$ dimension. The measurement represents the average diffusion of the water molecules contained in the voxel; however, the microscopic dimensions of the compartment in which the water is diffusing influence the measured values of diffusion tensor elements. The most popular parameter used to link the measured diffusion tensor elements to the geometric nature of the tissue is the fractional anisotropy, $F A$, which expresses the normalized variance of the eigenvalues [113]:

$$
F A=\frac{\sqrt{3}}{\sqrt{2}} \frac{\left|\bar{D}-\frac{1}{3} \operatorname{trace}(\bar{D}) I\right|}{|\bar{D}|}
$$


This parameter provides an indication of how anisotropic the diffusion is. In other words, it indicates how packed and/or ordered the axonal fibers are in each voxel.

\subsection{Human Brain}

The nervous system is a highly specialized tissue network that controls the interaction of the human body with its environment. The central nervous system, CNS, represents the largest part of the nervous system and is composed of the brain and the spinal cord. The main component of the CNS is the brain which regulates virtually all human activity. For many millennia the functions of the brain were unknown. A great deal of effort has been invested in understanding how the human brain works and much has been learned about its structure, anatomy and functionality. The brain is now considered as the primary organ responsible for the phenomena of consciousness, language and thought. It integrates and controls the autonomic functions in the body, performs cognition related with emotion and it is also responsible for the processing of vision and audition. It is quite embarrassing that the attempts to decipher the brain-code have not been entirely successful but evidence suggests that the brain acts as an ensemble of different modules that process specific kinds of information.

\subsubsection{Microstructural Organization}

Despite the large size and widespread distribution of the nervous system, it contains only two main categories of cells; nerve cells or neurons, which are the informationprocessing units and their supporting and sustaining cells called glia [114]. The human brain contains more than $10^{12}$ neurons and glial cells are 10 to 50 times more numerous than neurons 
[115]. The glial cells play an important role in guiding neuronal migration during development and serve a variety of metabolic and structural support roles. They come in two varieties: astrocytes and oligodendroglia. One of the major functional roles of the oligodendroglia cells is to fabricate the myelin sheath that encapsulates the axons and facilitates increased conduction of electrical impulses along the axons.

Neurons typically have four morphologically and functionally distinct regions: the cell body, the dendrites, the axons and the axon terminal region. They are bounded, like all cells, by a 75 to $100 \AA$ thick plasma membrane, which is composed primarily of lipids and proteins. This membrane is a semipermeable, bilayer membrane that allows certain substances to diffuse across it easily while restricting the transmembrane diffusion of others.

The cell bodies of human neurons range in size from 5 to $105 \mu \mathrm{m}$ [114]. The dendrites are true extensions of the cell body specialized in receiving information. They are fairly short highly branched and they arise smoothly from the cell body. In marked contrast with the dendrites, the cytology of axons is different from that of the cell soma. They arise abruptly from the soma and they not only participate in neuronal transmission but also transport the chemical substances synthesized in the cell body to axonal sites that can be as much as a $1.2 \mathrm{~m}$ away [114]. The neurons are in the business of conveying information; they communicate with each other through specialized junctions called synapses that normally occur between axon terminals and the dendrites of the receiving neuron. In the brain, the axons diameters range from about 0.1 to $25 \mu \mathrm{m}$ [115]. This is a significant a point of interest since their diameter relates to velocity of impulse conduction. The larger axons are wrapped with a myelin sheath. Myelin consists of spirally wrapped glial membranes where most of the cytoplasm has been squeezed out, serving as an electrical insulator. Some knowledge has been gained regarding 
the structure of the proteins and lipid bilayers of myelin. This structure is of some importance in understanding the MR imaging characteristics of myelin and will therefore be discussed in some detail.

Examination of myelin under an electron microscope [116] shows that the multiple layers of the sheath have a protein-lipid-protein-lipid structure [117]. Chemical investigations have determined that the lipid layers in the cell membrane are composed of a bimolecular layer of hydrocarbon chains, (cholesterol, phospholipids, and glycolipids). It appears that most of the glycolipid and cholesterol is in the outer layer of the membrane, exposed to the extracellular space, and the phospholipids are located exclusively in the inner side of the membrane. It is also known now that proteins comprise $19-20 \%$ of the dry weight of human myelin and lipids $78-80 \%$ such that for each molecule of myelin protein there are 186 lipid molecules, of which 111 are polar lipids and 75 are cholesterol [118].

\subsubsection{Macrostructural Organization}

The CNS is easily divided into grey matter and white matter. Grey matter refers to areas where there is a prevalence of cell bodies, glial cells and dendrites; it appears as a pinkish-grey color because it has an abundant blood supply. The cortex is an area of gray matter that forms a layered structure that covers most of the brain surface. There are also a few islands of gray matter deep inside the white matter of each cerebral hemisphere; collectively these are called basal ganglia. These small regions of the gray matter within the CNS are also frequently called cerebral nuclei [114].

White matter refers to areas consisting primarily of myelinated axons. The myelin sheaths consist mainly of lipid molecules and therefore they have a fatty, white appearance. In 
contrast to gray matter, the subdivision of the white matter has a complex organization. Nerve fibers (axons) are held together by several layers of tissue forming densely packed nerve bundles. A distinct group of nerve fibers is referred to as a peduncle or fasciculus. Bundles of fasciculi, along with the blood vessels that supply them, are called tracts. Their cross-sectional area could be of few $\mathrm{mm}$ in diameter and they have two-part names; the first part of the name refers to the location of the neuronal cell bodies from which these axons originate and the second part refers to the site where they terminate. For example, the corticospinal tracts connect the cortex to the spine. The merging and splitting of these nerve bundles and tracts makes the white matter from an anatomical point a view, a complex fascicular brain structure.

The tracts that make up the cerebrum's internal white matter are of three types: projection tracts, association tracts and commissural tracts [115]. Projection tracts are extensions of the ascending or sensory tracts, and the descending, or motor tracts, which mainly connect the cerebral cortex with the brainstem and spinal cord. These fibers often run in the coronal plane as they pass through the internal capsule. The fibers for special senses such as vision and olfaction connect the end organ with the appropriate cortical regions. They may also be in transverse or oblique transverse planes.

The association tracts are the most numerous of the cerebral tracts; they extend from one area of the cortex to another in the same hemisphere. There are short association fibers that connect adjacent gyri via subcortical $U$ fiber tracts and longer association fibers that connect cortical regions to more distant gyri in the same hemisphere of the brain.

Finally, the commissural tracts extend from one cortical location to the matching one in the other hemisphere. Most of their courses are seen in the coronal planes. The corpus callosum, (CC), is the major heterogeneous white-matter commissural tract and also the 
biggest tract in the human brain. The $\mathrm{CC}$ can be regarded as a broad band running from side to side crossing the brain midline. It connects the two cerebral hemispheres and provides communication between the cortical and subcortical neurons at the auditory, sensory and motor levels. It is a wide, flat, compact and transversely oriented collection of nerve fibers which consists, on average, of about $1.8 \times 10^{8}$ axons [119]. The posterior portion is called the splenium (SCC); the anterior part is called the genu (GCC); between the two is the body.

According to neuroanatomical tracing studies [120], the regional distribution of thin and large diameter fibers in the $\mathrm{CC}$ have complementary patterns. Thin fibers are densest in the GCC, the callosal region that connects the prefrontal cortex with higher-order sensory areas. Large-diameter fibers tend to be denser in regions connecting primary and secondary somatosensory, auditory and visual areas like the posterior part of the midbody of the $\mathrm{CC}$, and the SCC.

\subsection{Tissue Water Model}

About a century ago, a minority of scientists began to ask questions about the physical state and behavior of water in cells. It is natural to have long meditated on its role in life since water is ubiquitous and vital for the entire planet. The water control in the human body is rigorous. A deficiency in hydration of less than $5 \%$ is usually fatal. On the other hand, an increase of the water content in cells and tissues over the physiological limit changes protein activity and may trigger cell malfunctioning and death [121, 122].

One of the interesting questions that arises is whether cell water acts only as a solvent or whether it also has a specific structural and/or functional role to play. The problem is complicated by the lack of a complete understanding of the properties of liquid water itself. 
Water can act as individual isolated molecules, small clusters, or even much larger networks. It is believed that, some of water's unusual properties arise implicitly from its hydrogen-bonded network [123]. Each water molecule is capable of forming four hydrogen bonds implying that water can form a giant gel or cluster of water molecules. The water in a tissue can form tetrahedrally directed hydrogen bonds with neighbouring water molecules and can have dipolar and induced dipolar interactions with other molecules [124]. In particular, proteinwater interactions determine the 3D structures that proteins form in the cell.

It is surprising that even today much of the research dealing with biological molecules ignores the pivotal importance of water in life's machinery. This is far from the reality since water, as a unique liquid, controls, in an essential way, the structure, function and reactivity of many aspects of natural and biological systems. Water molecules occupy specific sites and form localized clusters with different macromolecular structures determined by their hydrogen-bonding capabilities.

A modern picture of the cell should emphasize four of its most important components, namely lipid membranes, nucleic acids, proteins and water, all fitted together spatially to make an overall integrated structure in a much more precise way than is usually imagined.

Physical techniques (e.g. X-ray [125, 126], neutron diffraction [127] or NMR [128], [129]) have not been able to provide the spatial and temporal resolution required to directly probe water molecules interacting with proteins or the polar head groups of phospholipids in aqueous solution. The current view of the structure and dynamics of the hydration of these macromolecular complexes is therefore based on more or less model-dependent interpretations of experimental data. Since the first studies invoking long-range hydration structures in 
biological systems [130] similar ideas have been championed in maintaining that the structures of so-called 'biological water' differ essentially from that of ordinary water [131, 132].

\subsubsection{Hydration Layers}

Water in cellular environments interacts strongly with polar protein side groups and phospholipid head groups. These water molecules form hydration layers around cellular macromolecules and along the membrane surfaces of the cell. Due to the polar nature of the water molecule the more positively charged oxygen atom will be electrostatically attracted to negatively charged side groups whereas the hydrogen atoms will be attracted to positively charged side groups. These electrostatically bonded water molecules, which form the first hydration layer, effectively act as polar side groups themselves and form a second hydration layer, and so on.

Water in the hydration layers is commonly referred to as bound water or structured water [122]. The use of the word "structured" comes from the intuitively attractive assumption of the existence of a degree of geometrical correlation (translational or orientational) with implications to the average time scale of its persistence. The word "bound" describes the interaction of water molecules with macromolecules leading to a decrease in the range of frequencies covered by the motions (translational and/or re-orientational) of the water molecules and possibly to increased anisotropy of those motions. The use of the labels "bound" and "free" in any particular system will, to some extent, be subjective. In general, however, a group of molecules experimentally distinguishable as having the greatest degree of motional restriction will often be labeled as "tightly bound" and the rest as "less strongly bound", "weakly bound" or "free" depending on the choice of reference. 


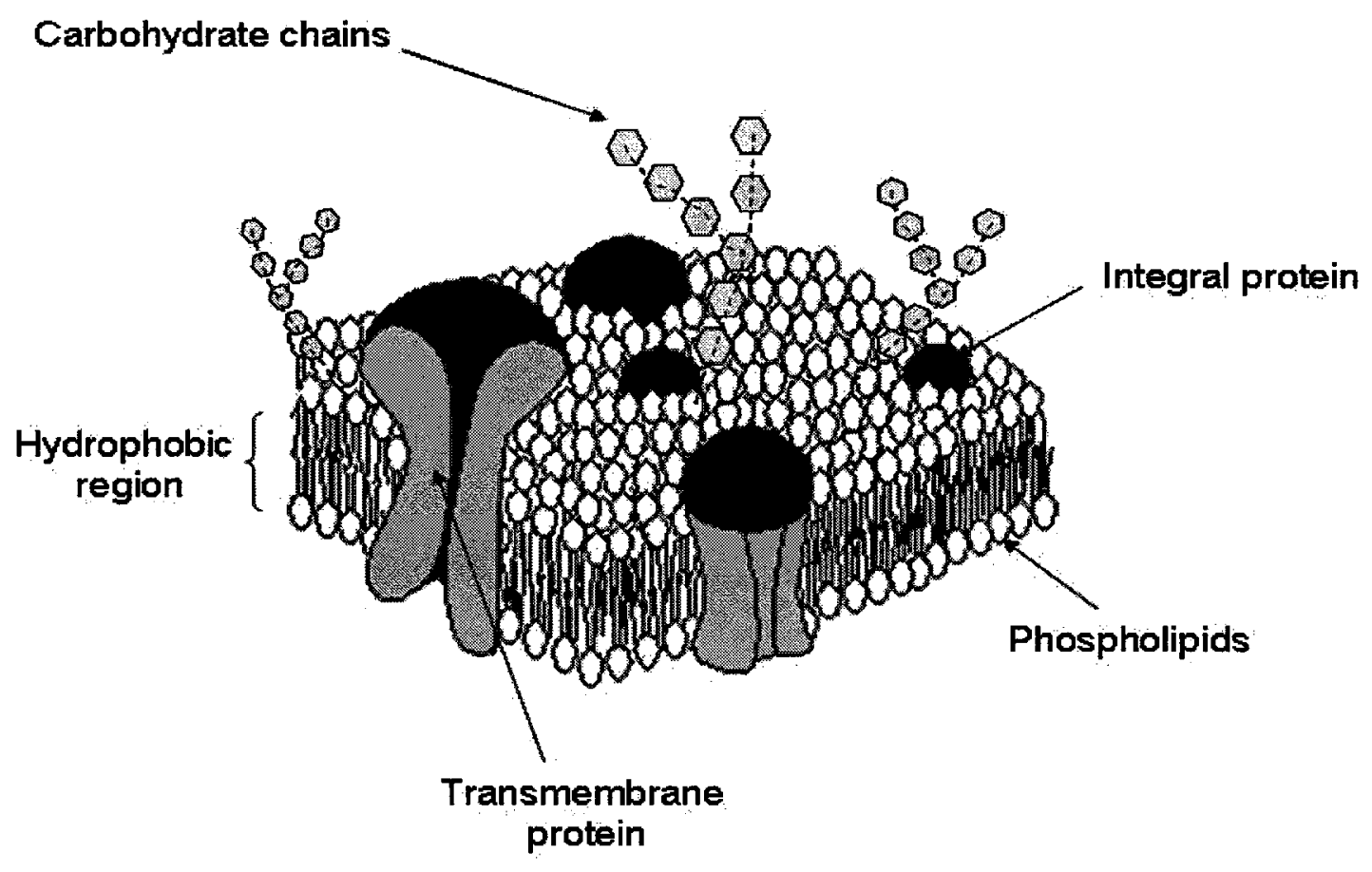

Figure 2.6: A schematic representation of the organization of the mosaic structure of the lipid bilayer which forms the membrane matrix. Globular protein molecules are partially embedded in the membrane and partially extending from it.

The plasma membranes that surround all animal cells are complex, heterogeneous protein-lipid assemblies which separate living cells from their extracellular surroundings; they are the interface between the cell and its environment. In normal tissue, water is in higher concentration outside the cell than it is inside the cell due to a low solute concentration of the extracellular environment [133]. The water concentration difference across the plasma membrane causes water to continuously move into the cell by osmosis [133] and the cell would swell and rupture if not counter-balanced in some way. On the assumption of no membrane resistance, the results of $[134,135]$ suggest that the hydration water tightly bound to the membrane surface has an important role to play in the overall decreased mobility of the 
tissue water across the membrane. The arrangement of local water molecules at the membrane interface is most probably the "barrier" that limits the diffusion rate rather than the membrane itself.

The particular geometry of membranes and the strong interactions of water with the polar lipid head groups and membrane proteins seem to dramatically affect the dynamic properties of water, especially at the membrane surface [67]. The most tightly bound water or the "first hydration layer" occurs adjacent to the membrane interface; however, additional layers of bound water which is motionally restricted compared to bulk water also occur beyond the first hydration layer, but the exact structural arrangement of water molecules in these outer hydration shells remains a matter of controversy. If separate layers of ordered structures exist and extend for a certain distance away from the membrane, this cooperative region strongly reinforces the concept that a large proportion of cellular water is osmotically unresponsive or perturbed from its bulk state. This is an important statement since the availability or nonavailability of free water in a cell controls the permeability of the membrane not only for the aquaporin channels [136] (intrinsic proteins embedded in the cell membrane that regulate the flow of water), but also for ionic species (e. g. $\mathrm{Na}^{+}, \mathrm{K}^{+}$) which successively interact with water [137].

Turning the attention to the dynamic aspect of hydration, this is generally specified by the type and rate of motion of the water molecules. Three types of motion should be distinguished: 1) proton exchange processes which limit the lifetime of a water molecule as a unit, 2) translational motion of the water molecule and 3) rotational motion of the water molecule itself. The lifetime of a water molecule at room temperature is of the order of milliseconds [138]. As this short period is, however, much longer than the timescales 
encountered during investigations into water's hydrogen bonding or hydration properties, the water molecule is usually treated as a permanent structure. Hydrogen bonds between water molecules allow for the establishment of a short lived network structure of water molecules. In bulk water, this network is very dynamic and rearranges itself every few picoseconds; the intermolecular hydrogen bond lifetimes are between 1 and $20 \mathrm{ps}$ and the time it takes for a new intermolecular hydrogen bond to form is about $0.1 \mathrm{ps}$ [123]. In this way, the hydrogen bonds "carry" information about solutes and hydrated surfaces over significant distances in liquid water. Failure to distinguish structural properties of the hydration water from its dynamic properties is a common source of confusion. Regrettably, the word dynamics is widely used to describe disorder and flexibility in biomolecular systems.

It has been appreciated for some time, as an important and longstanding biophysical problem, that water diffusion around macromolecular surfaces, including small globular proteins [139], multidomain proteins [140], DNA strands [130, 141] direct [142] and reverse micelles [143, 144] and lipid bilayers [145] is restricted with respect to bulk water. Furthermore, the perturbation of the tissue bulk water by the protein-lipid headgroups reacts back and perturbs the motion of these complexes themselves. The consistent dynamic picture emphasizes slower dynamics for all water molecules in the hydration shells. The first hydration layer shows "frozen" translational diffusion and suppressed rotational motion. The outer layer(s) exhibits water rotation correlation times that are similar to bulk water, and faster translational dynamics than the first hydration layer, but does not exhibit "bulk-like" translational diffusion values [146].

When a water molecule is near the surface of a charged macromolecular group, the free rotation of a water molecule is likely to be hindered by local geometric constraints and strong 
interactions with surface electrostatic attractive or repulsive forces. The water molecules will, as a result, tend to realign their dipoles radially with respect to the charged group. These interactions with the macromolecular side groups are stronger than the intermolecular hydrogen bonds between adjacent water molecules with the result that water diffusion will be locally decreased. This can be understood as a consequence of a lower local density of water, due to the fact that the water is effectively locally diluted by the inert, charged macromolecular group. This apparent decrease of molecular mobility of the water molecules in the hydration shell is correlated with a longer residence time in the bound states $[138,147]$. This does not necessarily indicate a particularly strong protein-water interaction but rather a topography that prevents the water molecule from exchanging by a cooperative mechanism. The remarkably fast molecular motions in normal liquid water result from cooperative rearrangements of the random hydrogen-bond network, allowing water molecules to rotate or translate without having to pass through a 'transition state', where all their hydrogen bonds are broken.

At somewhat greater distances from these charged groups, the electric field will orient the water dipoles to a lesser extent such that these molecules can be approached by other water molecules leading to an increase in the effective water density. This increase in global entropy will increase the translational and rotational motion of the water molecules. This effect may cause the cell to have only some of its water in an osmotically responsive state.

Although water comprises $70-80 \%$ of all living systems, there is a persistent difficulty of how to test the hydration models quantitatively. In fact, many direct physical measurements such as viscosity cannot be performed inside living cells, and most of the knowledge on protein-solvent interactions comes from studies done on proteins in vitro. For this reason, 
evidence for the correctness of the model must be sought in terms of the largest possible number of indirect experimental findings.

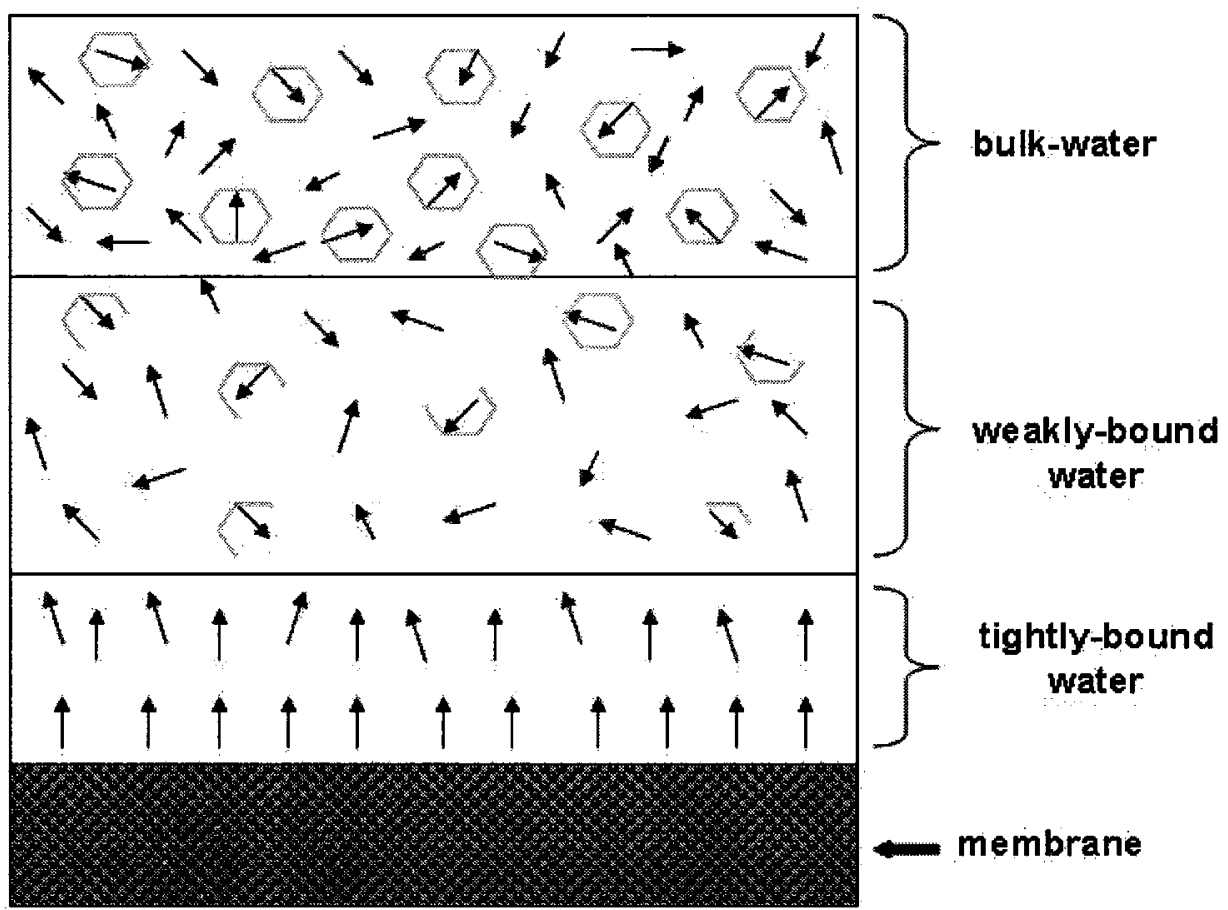

Figure 2.7: Three-layer model of water ordering near polar surfaces. The first few layers of molecules are highly ordered while, at some distance from the surface, the liquid has a "bulklike" structure. In between may be a disordered intermediate zone containing weakly-bound water.

Instead of quantifying the tissue water a priori, a three-layer model is proposed to characterize the $T_{2}$ relaxation and for human white matter tissue. The water content of the entire cell can be subdivided into three distinct structured water entities which possess considerable stability. The innermost structure, adjacent to the membrane surface is the tightly bound water; adjacent to the bound water is a mobile disordered zone consisting of weakly 
bound water, and finally, there is a more or less unaffected, "bulk-like" water structure (see Fig 2.7). As a note of caution, this three-layer model applies only to water near a polar surface which is able to induce a layer of dipoles at its interface. 


\section{Chapter 3}

\section{Noise Correction}

\subsection{Introduction}

Signal-to-noise ratio (SNR) considerations are of prime importance in MRI because the NMR signal is inherently weak. DWI is especially affected by low signal levels and a decreasing SNR as the diffusion weighting increases. For these low SNR situations the accuracy of the pixel intensity values is compromised and a correction for this effect is required.

After acquisition of the MR data in $k$-space the complex valued data consist of $A_{c}$, the true magnetic induction signal (i.e. the signal that would be obtained in the absence of noise) combined with the noise generated by the imaging electronics hardware and the subject [148], [149]. These raw MR signals are then Fourier transformed to obtain the image. Because the Fourier transform is a linear and orthogonal operation it will preserve the Gaussian 
characteristics of the noise [150]. Although all the information is contained in the real and imaginary parts of the Fourier transformed data, it is common practice to work with magnitude and phase data as they have more physical meaning. Furthermore, the magnitude data, unlike the real and imaginary data, are less sensitive to the effects of incidental phase variations due to RF flip angle inhomogeneity, non-centered sampling windows, etc. However, computation of a magnitude image is a nonlinear operation and the subsequent combination of real and imaginary data into a single magnitude image causes the Gaussian probability density function (PDF) of the pixels to be transformed into a Rician PDF [151].

In sections 3.2 and 3.3 of this chapter, the Rician PDF along with existing methods to correct for Rician bias will be reviewed. In section 3.3.3, an extension of one of the existing methods is presented which gives a superior implementation of one of these methods. Unfortunately, the published methods do not deal directly with the case where signal averaging in the image domain has been used in the acquisition of the images. This situation is considered in section 3.4. Simulation results are also presented in sections 3.3 and 3.4 to show that the method works. All of the Rician bias (RB) correction procedures presented here require a knowledge of the standard deviation of the background noise. In section 3.5 the sensitivity of the new algorithm proposed in section 3.3 to inaccuracies in this value is presented. Finally, in section 3.6, experimental data from a water phantom is presented to validate the technique. 


\subsection{PDF for MR Magnitude Image Pixel Intensities}

\subsubsection{The Rician Distribution}

The MR signal in the image domain can be written as

$$
s=A_{c}+n=A_{R}+i A_{I}+n_{R}+i n_{I}
$$

where $A_{R}$ and $A_{I}$ are the real and imaginary components of the true complex MR signal in the image domain, $A_{c}$, respectively, and $n_{R}$ and $n_{I}$ are real and imaginary components of $n$, the noise in the image domain, respectively. The noise components, $n_{R}$ and $n_{l}$, can both be characterized by a zero-mean Gaussian PDF with standard deviation $\sigma_{g}$ :

$$
P\left(n_{R, I}\right)=\frac{1}{\sqrt{2 \pi \sigma_{g}^{2}}} \exp \left(-\frac{n_{R, I}^{2}}{2 \sigma_{g}^{2}}\right),
$$

and the corresponding PDFs for $s_{R}$, and $s_{I}$ are:

$$
\begin{aligned}
& P\left(s_{R}\right)=\frac{1}{\sqrt{2 \pi \sigma_{g}^{2}}} \exp \left(-\frac{\left(A_{R}-s_{R}\right)^{2}}{2 \sigma_{g}^{2}}\right), \\
& P\left(s_{I}\right)=\frac{1}{\sqrt{2 \pi \sigma_{g}^{2}}} \exp \left(-\frac{\left(A_{I}-s_{I}\right)^{2}}{2 \sigma_{g}^{2}}\right),
\end{aligned}
$$

For a given measured signal, $s$, in the image domain the magnitude, $M$, can be written as:

$$
M=\sqrt{\left(A_{R}+n_{R}\right)^{2}+\left(A_{I}+n_{I}\right)^{2}}=\sqrt{\left(A \cos \phi+n_{R}\right)^{2}+\left(A \sin \phi+n_{I}\right)^{2}},
$$

where $A$ and $\phi$ are the magnitude and phase of $A_{c}$, the true, noiseless complex MR signal in the image domain.

The joint PDF for $s_{R}$ and $s_{I}$ corresponds to the product of the individual PDFs, since $s_{R}$ and $s_{I}$ are independent: 


$$
P\left(s_{R}, s_{I} \mid A, \phi, \sigma_{g}\right)=\frac{1}{2 \pi \sigma_{g}^{2}} \exp \left(-\frac{\left(A \cos \phi-s_{R}\right)^{2}}{2 \sigma_{g}^{2}}\right) \exp \left(-\frac{\left(A \sin \phi-s_{I}\right)^{2}}{2 \sigma_{g}^{2}}\right) .
$$

By expressing $s_{R}$ and $s_{I}$ in polar coordinates this PDF can be written:

$$
P\left(M, \varphi \mid A, \phi, \sigma_{g}\right)=\frac{M}{2 \pi \sigma_{g}^{2}} \exp \left(-\frac{(A \cos \phi-M \cos \varphi)^{2}}{2 \sigma_{g}{ }^{2}}\right) \exp \left(-\frac{(A \sin \phi-M \sin \varphi)^{2}}{2 \sigma_{g}{ }^{2}}\right)
$$

where $M$ and $\varphi$ are the magnitude and phase of $s$, the complex measured signal in the image domain and the Jacobian for this coordinate transformation is equal to $M$.

Integrating this expression over $\varphi$ and rearranging terms, it follows that:

$$
P_{M}\left(M \mid A, \phi, \sigma_{g}\right)=\frac{M}{2 \pi \sigma_{g}{ }^{2}} \int_{0}^{2 \pi} \exp \left[-\frac{1}{2 \sigma_{g}{ }^{2}}\left(A^{2}+M^{2}-2 A M \cos (\varphi-\phi)\right)\right] d \varphi
$$

where the subscripted $M$ indicates that the PDF is for the magnitude of the signal only. Since the integration is over a full cycle of the cosine, one can drop the $\phi$ and write:

$$
P_{M}\left(M \mid A, \sigma_{g}\right)=\frac{M}{2 \pi \sigma_{g}{ }^{2}} \exp \left[-\frac{1}{2 \sigma_{g}{ }^{2}}\left(A^{2}+M^{2}\right)\right] \int_{0}^{2 \pi} \exp \left[\frac{A M \cos \varphi}{\sigma_{g}{ }^{2}}\right] d \varphi
$$

The integral over $\varphi$ is equivalent to the integral representation of the 0 th order modified Bessel function of the first kind [152]:

$$
I_{0}(\alpha)=\frac{1}{2 \pi} \int_{0}^{2 \pi} \exp [\alpha \cos \varphi] d \varphi
$$

The resulting PDF is the so called Rician probability distribution function that characterizes magnitude data:

$$
P_{M}\left(M \mid A, \sigma_{g}\right)=\frac{M}{\sigma_{g}^{2}} \exp \left[-\left(M^{2}+A^{2}\right) / 2 \sigma_{g}^{2}\right] I_{0}\left(\frac{A M}{\sigma_{g}{ }^{2}}\right)
$$


This equation was first derived in 1944 by S. O. Rice [151] in the context of communication systems theory and later discussed by Henkleman [153] who derived closedform expressions in an MR context.

The shape of the Rice distribution depends on the SNR, which is here defined as the ratio $A / \sigma_{g}$. Fig. 3.1 shows the Rician PDF as a function of $M / \sigma_{g}$ for various values of the SNR.

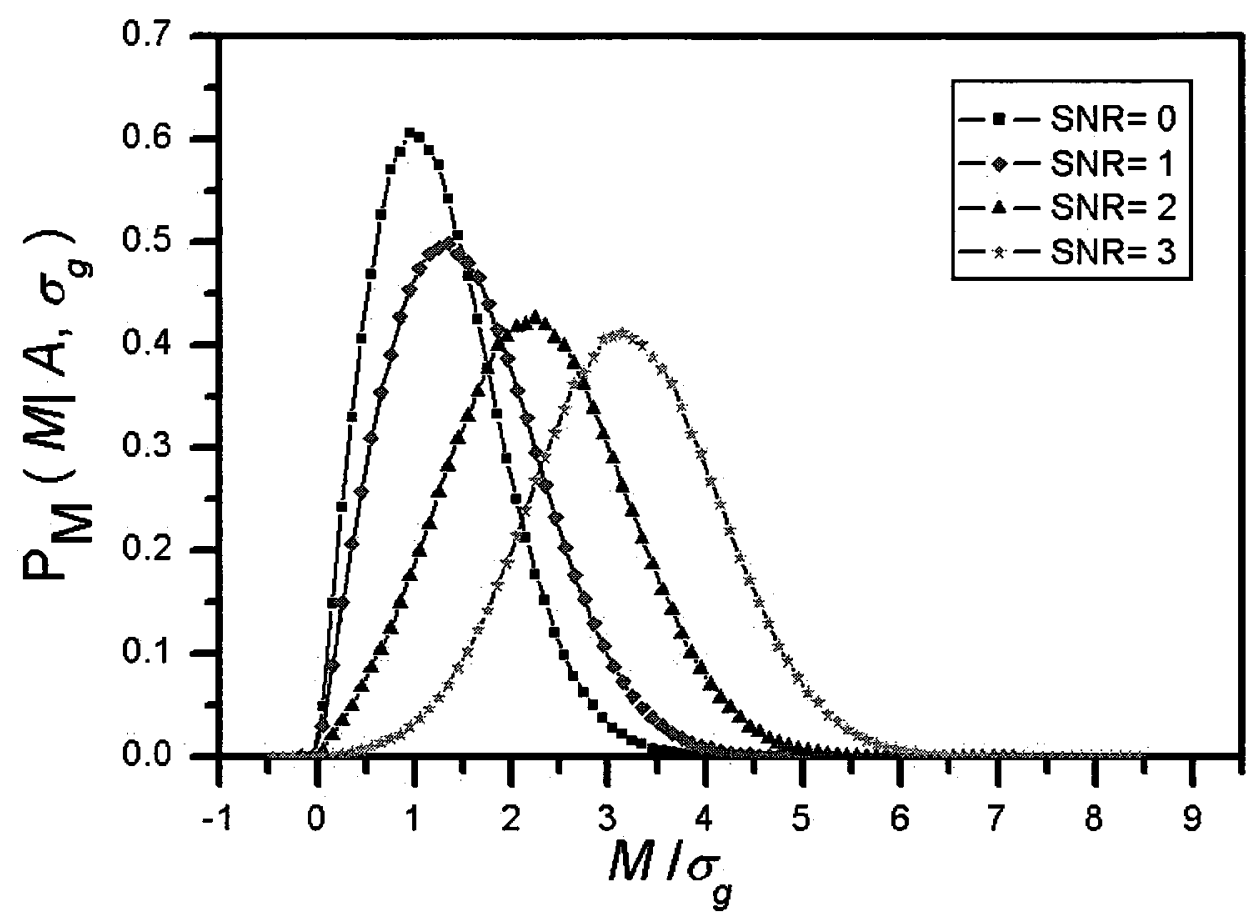

Figure 3.1: The Rician PDF for a magnitude image versus SNR. The convergence of the Rician distribution to a Gaussian in the high SNR limit and to a Raleigh distribution in the limit of a very low SNR can also be seen.

A special case of the Rician distribution characterizes image regions where only noise is present, the non-signal background area where $A=0$. For low SNR, the Rician PDF tends to a Rayleigh distribution, and Eq. (3.11) reduces to [154]: 


$$
P_{M}\left(M \mid \sigma_{g}\right)=\frac{M}{\sigma_{g}^{2}} \exp \left[-M^{2} / 2 \sigma_{g}^{2}\right]
$$

In another interesting limit, for SNR values greater than 3, the Rician distribution closely approximates a Gaussian distribution [154]:

$$
P_{M}\left(M \mid A, \sigma_{g}\right) \approx \frac{1}{\sqrt{2 \pi \sigma_{g}^{2}}} \exp \left[-\left(M-\sqrt{A^{2}+\sigma_{g}^{2}}\right)^{2} / 2 \sigma_{g}{ }^{2}\right] .
$$

The transition between the two limits of the Rician distribution can visually be appreciated in Fig. (3.1). However, for further discussion, the moments of the Rician PDF are required.

\subsubsection{The Moments of the Rician Distribution}

In general, the $v^{\text {th }}$ moment of the Rician PDF is defined as:

$$
E\left[M^{v}\right]=\int_{0}^{\infty} \frac{M^{v+1}}{\sigma_{g}^{2}} \exp \left[-\left(M^{2}+A^{2}\right) / 2 \sigma_{g}^{2}\right] I_{0}\left(\frac{M A}{\sigma_{g}^{2}}\right) d M
$$

where $E$ [ ] denotes the expectation operator. The solution of this equation can be expressed in terms of the confluent hypergeometric function of the first kind ${ }_{1} \mathrm{~F}_{1}\left[-\mathrm{v} / 2 ; 1 ;-A^{2} / 2 \sigma_{g}{ }^{2}\right][152]$.

The even moments of the Rice distribution, are simple polynomials:

$$
\begin{gathered}
E\left[M^{2}\right]=A^{2}+2 \sigma_{g}{ }^{2}, \\
E\left[M^{4}\right]=A^{4}+8 A^{2} \sigma_{g}{ }^{2}+8 \sigma_{g}{ }^{4} .
\end{gathered}
$$

The expressions for odd moments are much more complex. The first moment can be expressed as: 


$$
E[M]=\frac{1}{2 \sigma_{g}^{2}}\left\{\exp \left(-\frac{A^{2}}{4 \sigma_{g}^{2}}\right) \sqrt{\frac{\pi}{2}} \sigma_{g}\left[\left(A^{2}+2 \sigma_{g}^{2}\right) \mathrm{I}_{0}\left(\frac{A^{2}}{4 \sigma_{g}^{2}}\right)+A^{2} \mathrm{I}_{1}\left(\frac{A^{2}}{4 \sigma_{g}^{2}}\right)\right]\right\}=\mu_{R}
$$

where $I_{1}$ is the first order modified Bessel function of the first kind.

For completeness, the general expression for the moments of the limits of the Rice distribution should be reminded. For the Rayleigh distribution (i.e. the Rician PDF for SNR $\rightarrow$ 0) [155]:

$$
E\left[M^{v}\right]=\left(2 \sigma_{g}{ }^{2}\right)^{v / 2} \Gamma\left(1+\frac{v}{2}\right),
$$

with the mean and the variance for the Rayleigh distribution evaluated analytically:

$$
\begin{gathered}
E[M]=\sigma_{g} \sqrt{\frac{\pi}{2}}=\mu_{R 0}, \\
E\left[M^{2}\right]=(2-\pi / 2) \sigma_{g}{ }^{2} .
\end{gathered}
$$

For the Gaussian PDF (i.e. the Rician PDF for SNR $\rightarrow \infty$ ) [156]:

$$
E\left[M^{v}\right]=\sigma_{g}^{2(v-1)}\left[\frac{d^{v-1}}{d A^{v-1}}\left(A e^{\frac{A^{2}}{2 \sigma_{g}^{2}}}\right)\right] e^{-\frac{A^{2}}{2 \sigma_{g}^{2}}},
$$

where the standard deviation for the Gaussian PDF is $\sigma_{g}$ and the mean is:

$$
E[M]=\sqrt{A^{2}+\sigma_{g}^{2}}=\mu_{R \infty} .
$$

It should be noted that Eqs. (3.19) and (3.22) are the expected results since both relations could also be obtained from Eq. (3.17) as follows:

$$
\mu_{R 0}=\lim _{S N R \rightarrow 0} \mu_{R},
$$




$$
\mu_{R \infty}=\lim _{S N R \rightarrow \infty} \mu_{R} .
$$

\subsection{The Bias of the Rician Distribution}

The corresponding Rician distribution for low SNR values is asymmetric with a mean which is significantly greater than $A$, the magnitude of the signal in the absence of noise. When $A=0$, the measured signal equals the magnitude of the noise floor which is often referred to as the rectified noise floor (RNF). For $A>0$ the shift of the Rician PDF mean from $A$ will be called Rician bias, RB. Dealing with the RB effectively is a significant problem in quantitative MRI since all pixel values from MR magnitude images are biased by the noise to some extent.

\subsubsection{Review of Rician Bias Correction Techniques}

To obtain reliable quantitative information from MR images when the SNR is low a good strategy to remove the RB has to be sought. In principle, if there are hardware constraints, it is often possible in conventional imaging to improve the SNR by increasing the number of excitations, NEX, and to average the magnitude data over multiple acquisitions. However, this bias effect does not disappear when the average of several magnitudereconstructed signals is computed; the variance is reduced but the RB is unchanged.

Several techniques have been proposed to estimate the true signal intensity from noisy MR magnitude images. The noise correction techniques proposed in the literature provide different approaches for estimating the effect of noise and are usually employed after the image reconstruction has been completed. 
Henkelman [153] proposed a lookup table obtained by calculating numerically the signal change as a function of noise intensities. An unbiased estimator of the signal intensity was subsequently introduced by McGibney and Smith [157] and Miller and Joseph [158] based on Eq. (3.15) for E[ $\left.M^{2}\right]$. Miller and Joseph applied the correction to power images rather than magnitude images to avoid complications associated with the square root operation. The technique is of limited use since it can only be applied to power images and the distribution of the estimated signal intensity deviates strongly from a Gaussian distribution. The correction scheme of McGibney and Smith is for magnitude images rather than power images. Their method has its weakness at low SNR when the extracted MR signal, $A$, often has imaginary values. Gudbjartsson and Patz [154] have presented a similar but superior technique based on Eq. (3.22) in the following form:

$$
\tilde{A}_{G P}=\sqrt{\left|E[M]^{2}-\sigma_{g}^{2}\right|}
$$

where $\tilde{A}_{G P}$ is an estimator for $A$. This procedure does a good job of removing the noise bias at high SNR values but introduces a different bias by assigning the obtained imaginary values to their corresponding positive, real values. Until recently, this was the most commonly used method for removing RB in MRI.

Koay and Basser [159] have recently presented a novel analytically exact correction scheme based on a fixed point formula for SNR and a correction factor as a function of the SNR. This method will be discussed in detail in the following section.

Sijbers et al [160] have reported a RB removal technique, based on maximum likelihood theory, that performs comparably to the methods discussed above for SNR greater than 1 but they do not report results for SNR smaller than 1. Other approaches using maximum likelihood theory have also been presented [161] but these will not be considered here. 
Most of the RB correction approaches use an equation of the form:

$$
A^{2}=M^{2}-\Delta,
$$

where $M$ is the measured signal intensity and $\Delta$ is a positive correction term. The main problem with these methods is that when the SNR is low, $M^{2}$ will frequently be less than $\Delta$ leading to imaginary values for $A$. An improved RB correction method, discussed in Section 3.3.3 uses a binomial expansion to avoid the square root operation and to transform the equation into a linear correction. This correction technique works reasonably well for all SNR values and is an implementation of the theory developed by Koay and Basser which is presented in the next section.

\subsubsection{Koay and Basser Rician Bias Correction}

Koay and Basser [159] have recently proposed a new theoretical approach for estimating the signal intensity and the variance simultaneously from the MR magnitude data. They claim that this RB correction technique is analytically exact in the sense that this correction scheme is derived from two mathematical identities; they relate the variance of the Rician PDF to $\sigma_{g}{ }^{2}$ via a correction factor, $\xi(\theta)$ and derive a floating point expression that relates $\xi(\theta)$ to the true SNR for the measurement.

The variance of the Rician distribution which, by definition, is given by:

$$
\sigma_{R}^{2}=E\left[M^{2}\right]-E[M]^{2}
$$

can also be simply expressed as:

$$
\sigma_{R}^{2}=\xi(\theta) \sigma_{g}^{2}
$$

where $\theta=A / \sigma_{g}=$ SNR and, using Eqs. (3.15) and (3.17) it can be shown that $\xi(\theta)$ is given by: 


$$
\xi(\theta)=2+\theta^{2}-\frac{\pi}{8} \exp \left(-\frac{\theta^{2}}{2}\right)\left[\left(2+\theta^{2}\right) I_{0}\left(\frac{\theta^{2}}{4}\right)+\theta^{2} I_{1}\left(\frac{\theta^{2}}{4}\right)\right]^{2}
$$

Furthermore, since

$$
\sigma_{R}^{2} \equiv E\left[M^{2}\right]-E[M]^{2}=\xi(\theta) \sigma_{g}^{2},
$$

it can be seen, using Eqs. (3.15) and (3.17), that:

$$
2 \sigma_{g}^{2}+A^{2}-\mu_{R}^{2}=\xi(\theta) \sigma_{g}^{2}
$$

The fixed point formula for SNR is constructed based on Eq. (3.30) by factoring out $\sigma_{g}$ on both sides and by introducing $\theta$ to arrive at:

$$
\theta=\sqrt{\xi(\theta)\left[1+\frac{\mu_{R}^{2}}{\left.\sigma_{R}^{2}\right]-2}\right.}
$$

It should be observed that Eq. (3.31) establishes the correction factor $\xi(\theta)$ based on a one-toone correspondence between the true SNR, $\theta$, and the measured SNR, $\mu_{R} / \sigma_{R}$.

The fixed point formula of SNR described by Eq. (3.31) has a unique solution, as long as $\mu_{R}$ and $\sigma_{R}$ are known (which is not normally the case, in practice). Both $\theta$ and $\xi(\theta)$ can be simultaneously determined, in a iterative way, by starting off with an initial estimate for $\theta$. By defining $g(\theta)=\theta$, this iterative map will always converge [159] based on the composition of this function: $g(\theta)^{m}=g(\ldots g(g(\theta)) \ldots)$ (where $m$ is the number of iterative terms), as long as the lower bound for $g(\theta)$ is the limit at $\theta=0$. Eq. (3.31) is easy to solve when $\mu_{R}$ and $\sigma_{R}$ are given such that $\frac{\mu_{R}}{\sigma_{R}} \geq 1.9130$ (the lower bound) [159]. This correction scheme appears to be 
very promising and the iterative method for determining $\xi(\theta)$ is very elegant. Furthermore, the correction gives excellent results when accurate values for $\mu_{R}$ and $\sigma_{g}$ are used.

Eq. (3.31) can also be written more conveniently in terms of $A$ as:

$$
A^{2}=\mu_{R}^{2}+(\xi(\theta)-2) \sigma_{g}^{2}
$$

or,

$$
A=\sqrt{\mu_{R}^{2}-q^{2} \sigma_{g}^{2}}
$$

where

$$
q^{2} \equiv 2-\xi(\theta)
$$

It should be noted that $q$, as defined here, ranges from one to two since $\xi(\theta)$ ranges from zero to one [159].

Unfortunately, the theory presented above relies on knowing $\mu_{R}$ but, in practice, reliable values for $\mu_{R}$ are normally unavailable and approximations have to be introduced. The correction technique discussed in the following section is an extension of the theory presented here that is less sensitive to the use of approximate values for $\mu_{R}[162,163]$.

\subsubsection{Improved Rician Bias Correction Technique}

By replacing the square root operation in Eq. (3.33) with its equivalent binomial expansion, the equation becomes:

$$
A=\mu_{R}-\mu_{R}\left[\frac{1}{2}\left(\frac{q \sigma_{g}}{\mu_{R}}\right)^{2}+\frac{1}{8}\left(\frac{q \sigma_{g}}{\mu_{R}}\right)^{4}+\cdots\right]=\mu_{R}-\Delta \mu \quad \text { for } \frac{\mu_{R}}{q \sigma_{g}}>1
$$


Moreover, the condition that makes the binomial expansion possible will always be satisfied since $\mu_{R} \geq \mu_{R 0}=1.25 \sigma_{g}$.

Strictly speaking, Eq. (3.35) is mathematically equivalent to Eq. (3.33) and there is no real advantage to using one over the other when $\sigma_{g}$ and $\mu_{R}$ are known. However, by introducing the binomial expansion the square root operation is avoided in the favor of a linear equation with the correction term separated from the term to be corrected. Moreover, in this ideal case, the correction term $\Delta \mu$ is exactly equal to the RB.

An ideal noise correction scheme will be conceptually simple, accurate, easy to implement and can be applied to each individual image pixel separately. With these motivations, it becomes obvious that approximations have to be made in order to get reliable estimates for $A$, since all of the above equations are invalid for individual measurements; they involve statistical parameters such as mean and standard deviation.

If the approximation $\mu_{R} \rightarrow\left\langle M_{j}\right\rangle_{R n}$, where $\left\langle\ldots{ }_{R_{n}}\right.$ represents the mean calculated over $n$ Rician distributed data points, is introduced into Eq. (3.35) it becomes:

$$
\begin{aligned}
& \tilde{A}_{j, n^{\prime}, n}=\left\langle M_{j}\right\rangle_{R n^{\prime}}-\left\langle M_{j}\right\rangle_{R n}\left[\frac{1}{2}\left(\frac{q \sigma_{g}}{\left\langle M_{j}\right\rangle_{R n}}\right)^{2}+\frac{1}{8}\left(\frac{q \sigma_{g}}{\left\langle M_{j}\right\rangle_{R n}}\right)^{4}+\ldots\right]=\left\langle M_{j}\right\rangle_{R n^{\prime}}-\Delta M_{j, n} \\
& \text { for } \frac{\left\langle M_{j}\right\rangle_{R n}}{q \sigma_{g}}>1
\end{aligned}
$$

where tilde is used to indicate that $\widetilde{A}_{j, n^{\prime}, n}$ is an approximate value for the $A$ value of pixel $j$. In this expression the correction term (i.e. the second term) and the term to be corrected (i.e. the first term) are computed using an average over $n$ and $n^{\prime}$ Rician distributed data points, respectively. Eq. (3.36) is mathematically equivalent to Eq. (3.35) for $n, n \rightarrow \infty$ and can be 
used, for example, with nearest neighbour averaged signals where the signal to be corrected and the correction term need not be calculated using the same number of nearest neighbours. The only requirements are that the standard deviation of the noise, normally measured from the image background, is known and it is reasonable to treat neighboring pixels as being approximately equivalent.

It is useful to consider the first term to be the value of the pixel of interest, $M_{j}$, (i.e. $n$ ' $=1$ ) with the correction term calculated as an average over $n$ neighboring pixels. The RB for a given SNR should be independent of $M_{j}$, when the $M_{j}$ correspond to the same $A$ value. Therefore, the exact RB correction when $M_{j}=\mu_{R}$ should be the exact correction for all $M_{j}$ corresponding to the same $A$ value. For this special case it can be seen, from Eq. (3.35) that:

$$
\tilde{A}_{j}=M_{j}-\mu_{R}\left[\frac{1}{2}\left(\frac{q \sigma_{g}}{\mu_{R}}\right)^{2}+\frac{1}{8}\left(\frac{q \sigma_{g}}{\mu_{R}}\right)^{4}+\ldots\right]=M_{j}-\Delta \mu \quad \text { for }\left(\frac{\mu_{R}}{q \sigma_{g}}\right)>1 .
$$

The above equation gives an exact RB correction for each individual pixel for the case where $\sigma_{g}$ and $\mu_{R}$ are known. It should be pointed out that $\tilde{A}_{j}$ may not be a reliable estimate for $A$ when only one pixel (i.e. one $M_{j}$ value) is employed in the correction. A more reliable estimate of $A$ can be obtained if the values of $\tilde{A}_{j}$ are averaged over a large number of equivalent pixels that have been corrected individually using Eq. (3.37). This gives:

$$
\left\langle\tilde{A}_{j}\right\rangle_{R}=A=\left\langle M_{j}-\Delta \mu\right\rangle_{R}=\mu_{R}-\Delta \mu
$$

which demonstrates that Eq. (3.37) gives an exact RB correction for individual pixels when $\mu_{R}$ and $\sigma_{g}$ are both known. Note that 1) the distribution of pixel values due to the noise still exists, only the RB has been removed and 2) the shape and variance of the PDF are the same 
before and after the correction, only the mean has changed. The second property is not true for the previously introduced RB correction techniques.

For implementing this $\mathrm{RB}$ correction procedure on a computer it is more convenient to rewrite Eq. (3.36) as a recurrence relation in the following form:

$$
\begin{aligned}
& \tilde{A}_{j, n, n^{\prime}}=\left\langle M_{j}\right\rangle_{R n^{\prime}}-\left\langle M_{j}\right\rangle_{R n} \sum_{\mathrm{k}=1}^{\infty} a_{k-1} \frac{k-\frac{3}{2}}{k}\left(\frac{q \sigma_{g}}{\left\langle M_{j}\right\rangle_{R n}}\right)^{2}=\left\langle M_{j}\right\rangle_{R n^{\prime}}-\Delta M_{j, n} \\
& \text { for } \frac{\left\langle M_{j}\right\rangle_{R n}}{q \sigma_{g}}>1
\end{aligned}
$$

where $a_{0}=1$ and $a_{k-1}$ is the previous term in the series.

This linear RB correction technique, which uses a binomial expansion to linearize the correction equation, was first implemented using a fixed number of terms in the expansion [162]. This approach, which will be referred to as NC2, gave satisfactory results for SNR $\geq 1.5$ since the convergence of the binomial expansion was reached very quickly and there was no real advantage in keeping more than 3 terms in the expansion. However, the results for lower SNR were not as good since the convergence gets slower and it depends on the value of $A$. A closer inspection revealed that the number of terms required goes up drastically as the validity expression for the binomial expansion is approached. It will be shown in section 3.3.4, that using a convergence criterion works much better for small SNR.

All of the above equations refer to only the correction of pixels from MR images acquired with no signal averaging. Unfortunately, RB correction of signal averaged magnitude MR images has not been discussed in the literature. Signal averaging of magnitude pixel intensities causes the shape of the relevant PDF to change and it is not clear if the theory 
developed above applies to this case since it was developed assuming that the data follows a Rician distribution.

In the following section an improved implementation method for the RB correction technique introduced above is presented. It will be shown via simulations that this method, which uses a convergence criterion instead of a fixed number of terms in the expansion, is a far better approach. Its application to signal averaged images will also be presented.

\subsubsection{Validation of the Linear RB Correction Technique Using Simulations}

\subsubsection{Technique Validation using $\mu_{R}$ and $\sigma_{g}$}

The linear RB correction method introduced above should give excellent results and converge to the exact solution when the mean of the Rician distribution (i.e. when $\left\langle M_{j}\right\rangle_{R n} \rightarrow \mu_{R}$ ) is used in Eq. (3.39) to calculate the correction, $\left.\Delta M_{j, n}\right)$. This was tested with simulated PDFs for different values of $\theta=A / \sigma_{g}$ using 1) the true PDF mean, $\mu_{R}$, computed from Eq. (3.17) and 2) the mean of the simulated Rician PDF where $\sigma_{g}$ was set to 1 in both cases, for convenience.

The RB correction algorithm was tested using simulations. IDL 7.0 (IIT Visual Information Solutions) was used to generate uncorrected and corrected PDFs over a range of $\theta$ values from 0.0 to 3.0. For each simulation $N=1 \times 10^{6} \mathrm{MR}$ signal magnitudes, $M_{j}$, were calculated from the real and imaginary parts of the pixel signals as:

$$
M_{j}=<\left[\left(A+n_{j R}\right)^{2}+\left(A+n_{j l}\right)^{2}\right]^{1 / 2}>
$$


where $n_{j R}$ and $n_{j l}$ are Gaussian distributed random numbers chosen independently of each other using IDL's RANDOMN procedure.

The first step in the algorithm for the correction procedure is to find the value of $\xi(\theta)$. The iterative procedure proposed by Koay and Basser for solving Eq. (3.31) should be completely accurate when $\mu_{R}$ is used. Furthermore, it always converges for $\mu_{R} \geq \sqrt{\frac{\pi}{2}} \sigma_{g}=\mu_{R_{0}}$. The computation of $\xi(\theta)$ is considered to have converged when either the absolute value of the difference between two consecutive iterations is less than or equal to $1 \times 10^{-8}$ or the number of iterations exceeds 100 [159].

The next step in the RB correction algorithm is to calculate the terms of the binomial expansion. For the special case considered here, where $\left\langle M_{j}\right\rangle_{R n} \rightarrow \mu_{R}$, convergence is guaranteed; however, the convergence will be quite slow for small $\theta$ values. In the implementation of the RB correction algorithm, the series expansion is considered to have converged when either 1) the magnitude of the next term, in the expansion is less than $1 \times 10^{-9}$ or 2) the number of terms considered exceeds $2.5 \times 10^{5}$. It is necessary to consider a large number of terms in the series expansions since the series converges extremely slowly when $\mu_{R} \approx q \sigma_{g}$. Similarly, for the first convergence criterion, the value used for the cutoff term must be extremely small since there may be thousands of terms for the same order of magnitude when $\mu_{R} \approx q \sigma_{g}$. These convergence criteria will be discussed in more detail in section 3.3.4.2 below.

The results presented in Table 3.1 and Fig. 3.2 demonstrate that Eq. (3.39) gives the exact RB correction for individual pixels when $\mu_{R}$ and $\sigma_{g}$ are used. By introducing the binomial 


\begin{tabular}{ccccc}
\hline$\theta$ & $\frac{\mu_{R}}{\sigma_{g}}$ & $\left\langle\tilde{\theta}_{j}^{G P}\right\rangle_{R}$ & $\left\langle\tilde{\theta}_{j_{m=8}}^{N C 2}\right\rangle_{R}$ & $\left\langle\tilde{\theta}_{j}\right\rangle_{R}$ \\
\hline 0.00 & 1.2533 & 1.038 & 0.246 & 0.000 \\
0.50 & 1.3304 & 1.106 & 0.467 & 0.499 \\
1.00 & 1.5486 & 1.251 & 1.180 & 1.001 \\
1.50 & 1.8749 & 1.618 & 1.584 & 1.500 \\
2.00 & 2.2724 & 2.029 & 2.038 & 2.000 \\
2.50 & 2.7112 & 2.492 & 2.517 & 2.500 \\
3.00 & 3.1726 & 2.986 & 3.008 & 3.000 \\
\hline
\end{tabular}

Table 3.1: Estimates of $\theta$, as a function of the correct values, were obtained using: 1) the method proposed by Gudbjartsson and Patz [154] (column 3), 2) the binomial expansion implemented by Nezamzadeh [160] with 8 terms included (column 4) and 3) Eq. (3.39) where exact values for $\mu_{R}$ and $\sigma_{g}$ are used to calculate the correction (column 5).

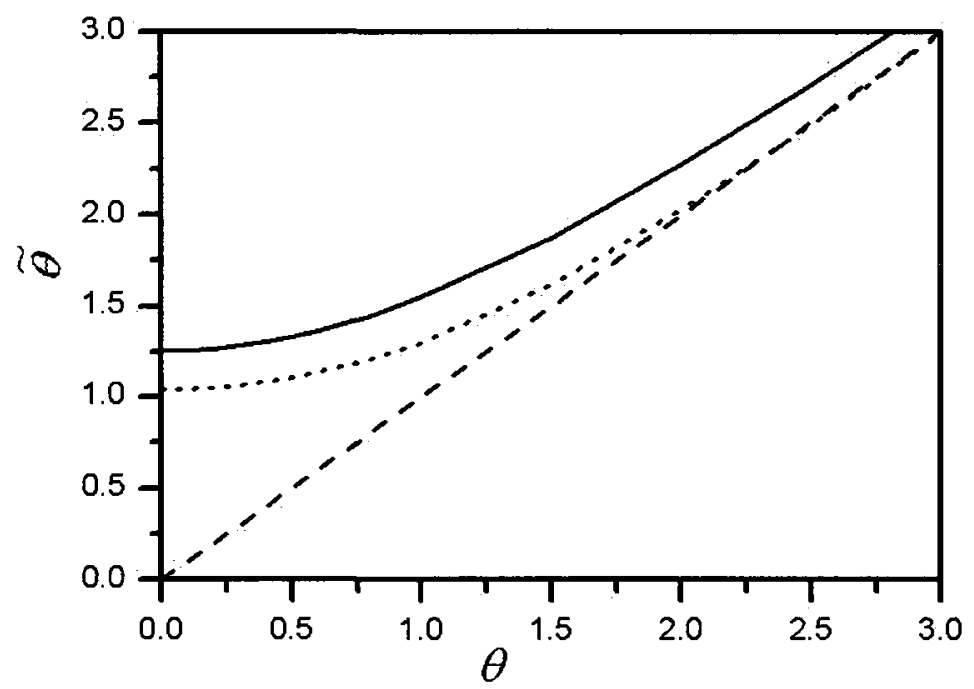

Figure 3.2: Estimates of $\theta$ plotted versus the correct values. The $\tilde{\theta}$ values were obtained by: 1) setting $\tilde{\theta}$ equal to $\mu_{R} / \sigma_{g}$ (solid line), 2) using the RB reduction method proposed by Gudbjartsson and Patz [154] (dotted line) and 3) using Eq. (3.39) to calculate the exact RB correction (dashed line) for the case where $\mu_{R}$ and $\sigma_{g}$ are both known exactly. 


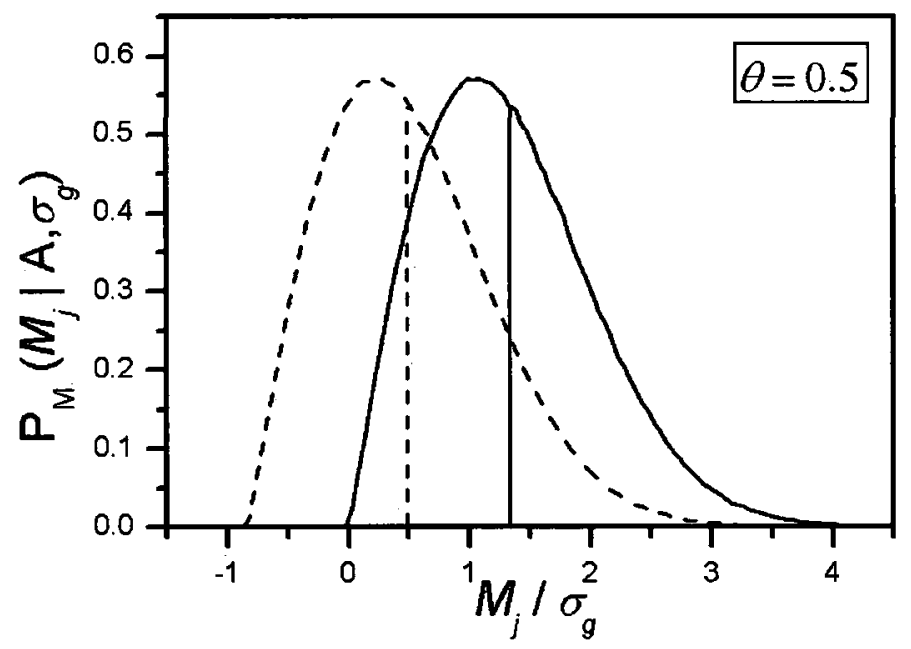

Figure 3.3: Simulated PDFs for before (solid line) and after (dotted line) RB correction using the binomial expansion method based on Eq. (3.39). For this case, the correct values for the PDF mean and standard deviation were used. The vertical lines show the corresponding PDF means. It should be noted that the shape of the PDF is undistorted and the mean of the corrected PDF is exactly equal to the assumed value, indicating a perfect RB correction.

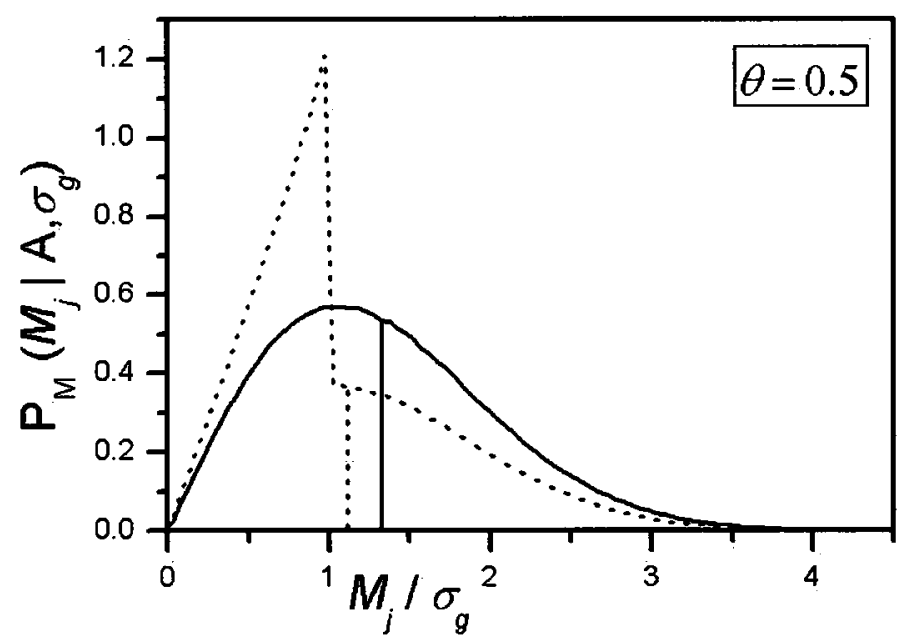

Figure 3.4: Simulated PDFs for before (solid line) and after (dotted line) RB correction using the method proposed by Gudbjartsson and Patz [154]. The vertical lines show the corresponding PDF means. 
expansion, the RB correction method presented here is transformed into a simple subtraction of the correction term, $\Delta \mu$, from the pixel intensity $M_{j}$, where $\Delta \mu$, expressed in terms of $\mu_{R}$ and $\sigma_{g}$, is exact. It should also be noted that the shape and variance of the PDF are the same before and after correction; only the mean value changes (see Fig. 3.3). These results show that the $\mathrm{RB}$ correction technique works as expected and that the implementation of the algorithm is working properly, even for extremely small $\theta$ values. This validates the technique, at least for the special case where $\mu_{R}$ and $\sigma_{g}$ are used. The results using the new linear RB correction method are much better and are significantly more accurate than those given by Gudbjartsson and Patz [154] (see Fig 3.2 and Table 3.1). Furthermore, with the Gudbjartsson and Patz method the PDF for the corrected data is drastically distorted (see Fig. 3.4).

The results obtained using the new algorithm presented here are also far superior to those reported by Nezamzadeh [163] especially at low $\theta$ values. Using 8 terms in the expansion is not enough to get full convergence for small $\theta$ values. In the same study, Nezamzadeh also suggested that the number of terms required for the binomial expansion will depend on the value of $A$ [163]. By instituting a convergence criterion, there is no need to arbitrarily set the number of terms to a fixed value where that value is satisfactory in some cases but not others.

\subsubsection{Convergence Criteria for the Linear RB Correction Technique}

A convergence criterion is often directly associated with the desired precision. If a precision of 0.001 is needed for the final result, then once the addition of a term in the expansion causes a change in the solution of less than this precision, the calculation is stopped. However, the convergence criterion used for Eq. (3.39) cannot be linked directly to the desired 
precision in this way since, as the argument in the expansion gets close to one, the series expansion may include many thousands of terms of the same order of magnitude which, when added together, can affect the answer. In the example below, the convergence criteria were set such that terms were added to the correction until either a term less than $1 \times 10^{-10}$ was encountered or the number of terms exceeded $10,000,000$.

It can be seen from Table 3.2 that, even though the difference between the $200,000^{\text {th }}$ and the $400,000^{\text {th }}$ term is of the order of $10^{-10}$, the difference in the estimates of $A$ for these two cases is of the order of $10^{-4}$. This effect is more acute as $A \rightarrow 0$.

\begin{tabular}{cccc}
\hline Term Number & Term value & $\Delta \mu$ & $\tilde{A}_{j}$ \\
\hline 200,000 & $3.1539452 \times 10^{-9}$ & 1.251733056 & 0.001581143 \\
400,000 & $1.1150828 \times 10^{-9}$ & 1.252196169 & 0.001118031 \\
600,000 & $6.0697325 \times 10^{-10}$ & 1.252401324 & 0.000912876 \\
800,000 & $3.9424038 \times 10^{-10}$ & 1.252523634 & 0.000790565 \\
$1,000,000$ & $2.8209532 \times 10^{-10}$ & 1.252607093 & 0.000707107 \\
$1,500,000$ & $1.5355315 \times 10^{-10}$ & 1.252736848 & 0.000577351 \\
$1,800,000$ & $1.1681181 \times 10^{-10}$ & 1.252787156 & 0.000527043 \\
$1,996,473$ & $9.9723892 \times 10^{-11}$ & 1.252813764 & 0.000500435 \\
\hline
\end{tabular}

a). 


\begin{tabular}{cccc}
\hline Term Number & Term value & $\Delta \mu$ & $\tilde{A}_{j}$ \\
\hline 200,000 & $3.1499321 \times 10^{-9}$ & 1.251731051 & 0.001583148 \\
400,000 & $1.1122469 \times 10^{-9}$ & 1.252193324 & 0.001120876 \\
600,000 & $6.0465921 \times 10^{-10}$ & 1.252397839 & 0.000916360 \\
800,000 & $3.9223764 \times 10^{-10}$ & 1.252519611 & 0.000794588 \\
$1,000,000$ & $2.8030515 \times 10^{-10}$ & 1.252602593 & 0.000711606 \\
$1,500,000$ & $1.5209380 \times 10^{-10}$ & 1.252731346 & 0.000582853 \\
$1,800,000$ & $1.1548086 \times 10^{-10}$ & 1.252781128 & 0.000533072 \\
$1,979,768$ & $9.9861235 \times 10^{-11}$ & 1.252805331 & 0.000508869 \\
\hline
\end{tabular}

b).

\begin{tabular}{cccc}
\hline Term Number & Term value & $\Delta \mu$ & $\tilde{A}_{j}$ \\
\hline 200,000 & $2.7768879 \times 10^{-9}$ & 1.251536210 & 0.001778290 \\
400,000 & $8.6440095 \times 10^{-10}$ & 1.251923258 & 0.001391241 \\
600,000 & $4.1426819 \times 10^{-10}$ & 1.252073543 & 0.001240956 \\
$1,000,000$ & $1.4925049 \times 10^{-10}$ & 1.252199539 & 0.001114961 \\
$1,199,812$ & $9.9747863 \times 10^{-11}$ & 1.252230220 & 0.001084279 \\
\hline
\end{tabular}

Table 3.2: RB correction and $\tilde{A}_{j}$ values obtained using Eq. (3.39) with $\left\langle M_{j}\right\rangle_{R n}$ set to the true mean, $\mu_{R}$, for given values of $A$ : a) $A=0.00$, b) $A=0.0001$, c) $A=0.001$, as a function of the number of terms in the binomial expansion used to calculate the correction. The value of the term corresponding to the term number given in the first column, is given in the second column. The size of the correction, $\Delta \mu$, is given in column 3. The convergence criteria used are discussed in the text. The convergence criteria were set such that terms were added to the correction until either a term less than $1 \times 10^{-10}$ was encountered or the number of terms exceeded 10,000,000.

Results for a second example, with an extended number of terms, are given in Table 3.4. The convergence criteria in this case were set such that terms were added to the 
correction until either a term less than $1 \times 10^{-14}$ was encountered or the number of terms exceeded $90,000,000$. With these constraints an accuracy of less than $14 \%$ was obtained for $A$ $=0.0001$. For $A>0.0001$ the percent error was less than $0.002 \%$. For $A \leq 0.001$ the results using the binomial expansion were not quite as good as the values obtained directly from Eq. (3.33), which it will be called $A_{K, B}$; for $A>0.001$ the results were identical. For $A=0$, the percent error could not be calculated but the deviation was very small $7.45 \times 10^{-5}$, and compares well with the $A_{K B}$ result of $2.58 \times 10^{-8}$.

\begin{tabular}{cccccc}
\hline$A$ & $A_{K, B}$ & $\tilde{A}_{j}$ & $\Delta \mu$ & Term Number & \%error \\
\hline 0.0000000 & $2.5809566 \times 10^{-8}$ & $7.4535604 \times 10^{-5}$ & 1.2532396 & 90000000 & - \\
0.0001000 & 0.00010002 & 0.00011359 & 1.2532006 & 90000000 & 13.6 \\
0.0010000 & 0.00100001 & 0.00100018 & 1.2523144 & 10545965 & 0.00187 \\
0.0100000 & 0.01000001 & 0.01000001 & 1.2433455 & 199009 & $1.24 \times 10^{-05}$ \\
0.0500000 & 0.05000001 & 0.05000001 & 1.2040974 & 10719 & $1.97 \times 10^{-06}$ \\
0.1000000 & 0.10000000 & 0.10000000 & 1.1564455 & 2986 & $2.17 \times 10^{-06}$ \\
0.2000000 & 0.20000000 & 0.20000000 & 1.0658161 & 827 & $4.47 \times 10^{-07}$ \\
0.3000000 & 0.30000000 & 0.30000000 & 0.9813563 & 391 & $7.00 \times 10^{-07}$ \\
0.5000000 & 0.50000000 & 0.50000000 & 0.8304474 & 154 & $9.74 \times 10^{-08}$ \\
1.0000000 & 1.00000000 & 1.00000000 & 0.5485725 & 47 & $1.20 \times 10^{-07}$ \\
1.5000000 & 1.50000000 & 1.50000000 & 0.3749357 & 26 & $3.89 \times 10^{-08}$ \\
2.0000000 & 2.00000000 & 2.00000000 & 0.2723834 & 18 & $2.18 \times 10^{-09}$ \\
3.0000000 & 3.00000000 & 3.00000000 & 0.1725773 & 13 & $2.49 \times 10^{-10}$ \\
\hline
\end{tabular}

Table 3.3: Values of $\tilde{A}_{j}$ and $A_{K, B}$ calculated using the true mean, $\mu_{R}$, for values of $A$ ranging from 0.00 to 3.00. The number of terms required for convergence is given in column 5 and the percent error for each case is given in the last column. The convergence criteria for this case were set such that terms were added to the correction until either a term less than $1 \times 10^{-14}$ was encountered or the number of terms exceeded $90,000,000$. 
Although this method is not quite as good as the direct method, it still gives excellent results. However, to get this level of accuracy it requires too much computer time. For example, for $A=0$, all 90 millions terms were used and the computation took around 3 minutes of computer time. Before this algorithm is used in practice, more reasonable expectations for the precision and the amount of computer time that can be tolerated for a single calculation must be established. This will be considered below.

\begin{tabular}{cccccc}
\hline$A$ & $A_{K, B}$ & $\tilde{A}_{j}$ & $\Delta \mu$ & Term Number & \%error \\
\hline 0.0000000 & $2.5809566 \times 10^{-8}$ & 0.00141421 & 1.2518999 & 250000 & - \\
0.0001000 & 0.00010000 & 0.00141660 & 1.2518977 & 250000 & 1320 \\
0.0010000 & 0.00100011 & 0.00163351 & 1.2516810 & 250000 & 63.4 \\
0.0100000 & 0.01000001 & 0.01001417 & 1.2433313 & 50482 & 0.142 \\
0.0500000 & 0.05000001 & 0.05000066 & 1.2040967 & 4335 & 0.00132 \\
0.1000000 & 0.10000000 & 0.10000017 & 1.1564453 & 1360 & 0.000171 \\
0.2000000 & 0.20000000 & 0.20000004 & 1.0658160 & 413 & $2.18 \times 10^{-05}$ \\
0.3000000 & 0.30000000 & 0.30000002 & 0.9813562 & 204 & $7.13 \times 10^{-06}$ \\
0.5000000 & 0.50000000 & 0.50000001 & 0.8304475 & 85 & $1.35 \times 10^{-06}$ \\
1.0000000 & 1.00000000 & 1.00000000 & 0.5485724 & 27 & $3.04 \times 10^{-07}$ \\
1.5000000 & 1.50000000 & 1.50000000 & 0.3749357 & 16 & $6.09 \times 10^{-07}$ \\
2.0000000 & 2.00000000 & 2.00000000 & 0.2723835 & 11 & $1.93 \times 10^{-08}$ \\
3.0000000 & 3.00000000 & 3.00000000 & 0.1725773 & 8 & $2.36 \times 10^{-09}$ \\
\hline
\end{tabular}

Table 3.4: Values of $\tilde{A}_{j}$ and $A_{K, B}$ calculated using the true mean, $\mu_{R}$, for values of $A$ ranging from 0.00 to 3.00. The number of terms required for convergence is given in column 5 and the percent error for each case is given in the last column. The convergence criteria for this case were set such that terms were added to the correction until either a term less than $1 \times 10^{-9}$ was encountered or the number of terms exceeded 250,000 . 
The size of the minimum term used in the convergence criteria was increased gradually from $1 \times 10^{-14}$ to $1 \times 10^{-12}$ while the maximum number of terms was reduced to 5 million. This still gives good results for $A=0.001$. For $A=0.00$ the deviation was $\sim 1 \times 10^{-4}$ and $10 \mathrm{~s}$ of computer time were required. When the minimum term was increased to $1 \times 10^{-8}$ the expansion used 30,000 terms for $A=0.01$. A good compromise appears to be to set the size of the minimum term to $1 \times 10^{-9}$ and the maximum number of terms to 250,000 as the limits for the series expansion to get good accuracy for values of $A>0.01$. Results with these convergence criteria are given in Table 3.4. These settings give a deviation of 0.0014 for $A=0.00$ with a calculation time of less than $0.5 \mathrm{~s}$. These are the convergence criteria used when the RB correction was applied to the experimental data and they were found to work well.

The linear RB correction method using a binomial expansion has been shown to work very well but since it is not better than the direct method proposed by Koay and Basser when $\mu_{R}$ and $\sigma_{g}$ are known accurately; there is no real advantage to using it for this situation. Unfortunately, for most situations of interest in vivo, reliable $\mu_{R}$ values are not available. To deal with these situations, a nearest neighbor average gives a useful approximation to $\mu_{R}$ as long as a small group of adjacent pixels can be considered approximately equivalent. This is considered in the next section.

\subsubsection{Linear RB Correction Using Nearest Neighbour Averaging}

In this section the performance of the proposed RB correction algorithm is evaluated as a function of $n$, the number of nearest neighbor pixels averaged together. The results given here were computed using Eq. (3.39) where the first term was taken to be a single pixel value (i.e. $\left\langle M_{j}\right\rangle_{R n^{\prime}} \rightarrow M_{j}, n^{\prime}=1$ ) and the correction term $\left\langle M_{j}\right\rangle_{R n}$ was calculated in the simulation 
as the mean over $n$ consecutive $M_{j}$ values generated for a given $A$ using Eq. (3.40). Once $<M_{j}$ $>_{R n}$ is evaluated, the value of $\xi(\theta)$ that corresponds to this signal amplitude is determined as long as $\left\langle M_{j}\right\rangle_{R n} \geq \sqrt{\frac{\pi}{2}} \sigma_{g}=\mu_{R_{0}}$. When this validity condition is violated, the RB correction algorithm sets $\xi(\theta)=\xi(0)$ which is equivalent to setting $q^{2}(\theta)=q^{2}(0)=\pi / 2$. Once $\xi(\theta)$ has been evaluated the next step is to check the validity condition for the binomial expansion (see Eq. (3.36)). When this condition is satisfied the correction term is computed. When it is violated, the algorithm sets $\Delta M_{j, n}=\sqrt{\frac{\pi}{2}} \sigma_{g}$ which is the theoretical correction value for $A=0$. This may not be completely satisfactory but it will avoid situations that either involve the square root of a negative number or, equivalently, a diverging series. Once $\Delta M_{j, n}$ has evaluated, $\widetilde{A}_{j, 1, n}$ can be determined.

The results for $\tilde{A}_{j, 1, n}$, averaged over the full PDF, are presented in Table 3.5 for $n=5$, $9,25,100,625$ and 1000 . The estimated $\left\langle\tilde{A}_{j, 1, n}\right\rangle_{R}$ values approach the true $A$ as the SNR increases: for $n \geq 9$ and $A \geq 1$ the correction is accurate to within $10 \%$ and for $n=100$, the results are accurate to within $13 \%$ for $A \geq 0.2$. For large values of $n$ the correction improves considerably since the variance of the $\operatorname{PDF}(n)$ is smaller relative to the original $\operatorname{PDF}$ by a factor of $n$.

Averaging over one pixel (i.e. $n=1$ ) was also investigated but the results are not included here. In this case the pixel value itself is assumed to be equal to the PDF mean in the calculation of the correction term. This is clearly a very poor approximation, since an individual pixel value may be far from the PDF mean, which leads to poor results. 


\begin{tabular}{ccccccc}
\hline \multirow{2}{*}{$A$} & \multicolumn{5}{c}{$\left\langle\tilde{A}_{j, 1, n}\right\rangle_{R}$} \\
& $n=5$ & $n=9$ & $n=25$ & $n=100$ & $n=625$ & $n=1000$ \\
\hline 0.00 & 0.285 & 0.257 & 0.214 & 0.161 & 0.108 & 0.099 \\
0.10 & 0.291 & 0.266 & 0.223 & 0.174 & 0.126 & 0.116 \\
0.20 & 0.310 & 0.286 & 0.249 & 0.208 & 0.180 & 0.178 \\
0.30 & 0.342 & 0.320 & 0.292 & 0.266 & 0.268 & 0.274 \\
0.40 & 0.385 & 0.369 & 0.352 & 0.349 & 0.381 & 0.388 \\
0.50 & 0.440 & 0.433 & 0.428 & 0.450 & 0.492 & 0.494 \\
0.60 & 0.507 & 0.507 & 0.520 & 0.563 & 0.594 & 0.596 \\
0.70 & 0.583 & 0.593 & 0.624 & 0.675 & 0.697 & 0.697 \\
0.80 & 0.671 & 0.691 & 0.735 & 0.783 & 0.799 & 0.799 \\
0.90 & 0.764 & 0.794 & 0.846 & 0.890 & 0.899 & 0.899 \\
1.00 & 0.869 & 0.902 & 0.958 & 0.992 & 0.999 & 0.999 \\
1.20 & 1.084 & 1.127 & 1.175 & 1.195 & 1.200 & 1.200 \\
1.40 & 1.309 & 1.349 & 1.387 & 1.396 & 1.400 & 1.400 \\
1.50 & 1.425 & 1.459 & 1.490 & 1.496 & 1.500 & 1.500 \\
1.75 & 1.701 & 1.726 & 1.742 & 1.751 & 1.750 & 1.750 \\
2.00 & 1.968 & 1.986 & 1.996 & 1.998 & 1.999 & 1.999 \\
2.50 & 2.487 & 2.493 & 2.497 & 2.500 & 2.500 & 2.500 \\
3.00 & 2.994 & 2.996 & 2.999 & 2.999 & 3.000 & 3.000 \\
\hline
\end{tabular}

Table 3.5: $\left\langle\widetilde{A}_{j, 1, n}\right\rangle_{R}$ values for $A$ ranging from 0 to 3 for several $n$ values and NEX $=1$. For all simulations $\sigma_{g}$ was set to 1 .

It should be noted that this proposed RB correction technique works reasonably well even in the limit of low SNR, but requires, at least 9 pixels in the nearest neighbor average in order to have a reasonable approximation of the PDF mean. Unfortunately, when dealing with 
real images, it may not always be possible to average over a large number of nearest neighbors for the calculation of the correction term since this implies an equally large region where adjacent pixels can reasonably be assumed to be equivalent.

\subsection{Rician Bias Correction Technique for Magnitude Averaged Signals}

One of the well known methods for increasing the SNR in MR measurements is signal averaging, the collection and averaging together of several images pixel by pixel. For each pixel in an image, the mean of the magnitude pixel data over a set of NEX measurements is calculated as follows:

$$
<M>_{N E X}=\frac{1}{N E X} \sum_{i=1}^{N E X} M_{i}=<\left[\left(A_{R}+n_{i R}\right)^{2}+\left(A_{I}+n_{i l}\right)^{2}\right]^{1 / 2}>_{N E X}
$$

where $M_{i}$ represents the magnitude of the $i^{t h}$ measurement calculated from the real, $A_{R}+n_{i R}$, and imaginary, $A_{I}+n_{i l}$, parts of the pixel signal. It is assumed here that each signal comes from the same voxel and, therefore, $A_{c}$ does not change from measurement to measurement.

Averaging in the image domain reduces the effective noise but not the Rician bias since the averaged value $\langle M\rangle_{N E X}$ is still corrupted by the nonlinear contribution from the noise arising from the magnitude calculation. For this reason, MR image quality for low SNR images cannot be increased indefinitely by signal averaging [164] and the RB remains a significant problem. Furthermore, since acquiring a large number of averages, in practice, requires an unreasonably long time, one is normally limited to working with a small number of averages. 
For all of the reasons mentioned above, at low SNR, a post-processing scheme to correct for the Rician bias combined with a limited amount of signal averaging is required. Since the improved linear RB correction technique presented above deals with Rician distributions and requires reliable values for the mean of the Rician PDF, this raises the question as to whether or not the RB correction scheme works properly when NEX $>1$, since signal averaging changes the nature of the PDF.

\subsubsection{The PDF of Magnitude Averaged Signals}

Increasing the number of averages, NEX, for magnitude images causes the Rician PDF to evolve towards a Gaussian PDF. This raises questions about the use of all of the RB correction techniques introduced above since they assume the data distribution corresponds to a Rician PDF. More precisely, Eqs. (3.36) and (3.39) are derived from the equation for the mean of the Rician PDF. Is the mean value affected by this evolution from a Rician PDF to a Gaussian PDF? If the mean of the PDF remains the same as NEX increases the RB correction techniques presented above should still be valid.

To observe the change in the Rician PDF as a result of averaging, simulated noisy MR signal magnitudes were generated for $A=0,1$ and 3 and $\mathrm{NEX}=1,3,6$ and 9. For $A=0$, and NEX $=1$, a Rayleigh PDF is obtained. As NEX increases the shape of the PDF changes to Gaussian-like (see Fig. 3.5), but the mean remains the same. It is somewhat surprising that the mean is not affected as the PDF evolves from the strongly asymmetric Rayleigh distribution to the highly symmetric Gaussian distribution. Additional simulation results for $A>0$ show that the PDF shape again changes from Rician to Gaussian-like as NEX increases and that the PDF mean again does not change as NEX increases. This is shown in Table 3.6 where the PDF 
mean values can be seen to be independent of NEX. Although the PDF shape deviates from the original Rician shape as NEX increases, the mean is the same for all $A$ values considered. This gives confidence that RB correction techniques based on Eq. (3.17) remain valid for NEX $>1$.

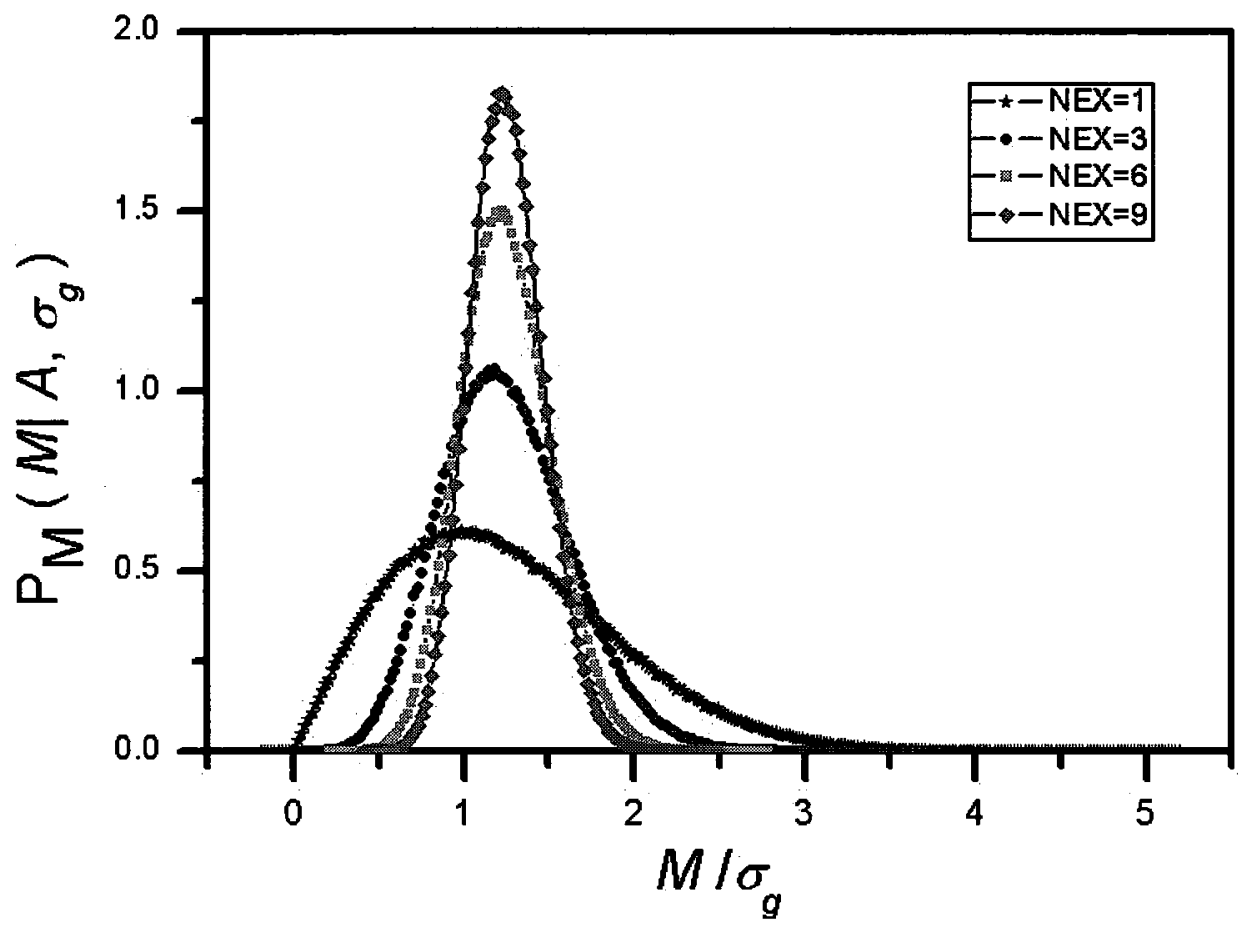

Figure 3.5: PDFs for $A=0$ and $N E X=1,3,6$ and 9. The mean for all of these PDFs is 1.25.

Another important consequence of the PDF mean remaining the same as NEX increases, in spite of the PDF shape change, is that the value of $\sigma_{g}$ used in the RB corrections is also unaffected because it is calculated from the signal mean in an area of the image background where $A$ can reasonably be assumed to be zero. This is an important result since an accurate value for $\sigma_{g}$ is required in order to perform this RB correction. 


\begin{tabular}{cccccccccccccc}
\hline \multicolumn{10}{c}{ PDF mean } \\
\hline$A$ & 0.00 & 0.20 & 0.50 & 0.75 & 1.00 & 1.25 & 1.50 & 1.75 & 2.00 & 2.25 & 2.50 & 1.25 \\
$\mathrm{NEX}=1$ & 1.253 & 1.266 & 1.331 & 1.424 & 1.548 & 1.701 & 1.875 & 2.067 & 2.272 & 2.488 & 2.712 & 3.171 \\
$\mathrm{NEX}=3$ & 1.253 & 1.266 & 1.330 & 1.424 & 1.549 & 1.700 & 1.876 & 2.067 & 2.273 & 2.487 & 2.712 & 3.172 \\
$\mathrm{NEX}=6$ & 1.253 & 1.266 & 1.330 & 1.423 & 1.548 & 1.701 & 1.875 & 2.067 & 2.272 & 2.488 & 2.711 & 3.172 \\
$\mathrm{NEX}=9$ & 1.253 & 1.266 & 1.331 & 1.423 & 1.548 & 1.701 & 1.875 & 2.068 & 2.273 & 2.488 & 2.712 & 3.173 \\
\hline
\end{tabular}

Table 3.6: Estimates of the PDF mean for $A$ ranging from 0 to 3 and $\mathrm{NEX}=1,3,6$ and 9 .

Note that performing signal averaging before the magnitude is calculated will decrease $\sigma_{g}$ by the square root of the number of averages. Once the magnitude is taken the PDF switches to a Rician PDF corresponding to a new higher effective SNR. The mean of the relevant PDF for the signal averaged signals will then correspond to the value for the new SNR. Thus, the theory as outlined above for NEX $=1$ should apply directly as long as the standard deviation used is the signal averaged value. Conversely, when the averaging is performed after the magnitude is calculated, the shape of the PDF changes but the PDF mean is not affected by the averaging. These are two quite distinct scenarios.

Better results can be obtained when $n$ and NEX are both greater than one. Simulation results show that the RB correction improves as a function of $n \times$ NEX and also converges to the correct result (see Table 3.7). The results are also the same for different combinations of $n$ and NEX that give the same product. For example, the same result was obtained for the following cases: $n=9$ with $\operatorname{NEX}=1 ; n=3$ with $\operatorname{NEX}=3$ and $n=1$ with NEX $=9$. This confirms the expected result since a nearest neighbor average over $n$ nearest neighbors which have been signal averaged NEX times, corresponds to an average over $n \times$ NEX Rician 91 
distributed values. This corresponds to the very common situation where the signal correction involves a combination of nearest neighbour averaging and signal averaging in the image domain.

\begin{tabular}{ccccccccccc}
\hline$A$ & & & \multicolumn{1}{c}{$\left\langle\tilde{A}_{j, 1, n}\right\rangle_{R}$} \\
& $3 \times 3$ & $9 \times 1$ & $5 \times 5$ & $25 \times 1$ & $7 \times 7$ & $49 \times 1$ & $10 \times 10$ & $100 \times 1$ & $25 \times 25$ & $625 \times 1$ \\
\hline 0.00 & 0.259 & 0.257 & 0.214 & 0.214 & 0.188 & 0.187 & 0.162 & 0.161 & 0.107 & 0.108 \\
0.10 & 0.265 & 0.266 & 0.223 & 0.223 & 0.197 & 0.198 & 0.173 & 0.174 & 0.125 & 0.126 \\
0.20 & 0.287 & 0.286 & 0.249 & 0.249 & 0.226 & 0.227 & 0.208 & 0.208 & 0.179 & 0.180 \\
0.30 & 0.321 & 0.320 & 0.292 & 0.292 & 0.276 & 0.276 & 0.266 & 0.266 & 0.269 & 0.268 \\
0.40 & 0.369 & 0.369 & 0.352 & 0.352 & 0.346 & 0.347 & 0.349 & 0.349 & 0.380 & 0.381 \\
0.50 & 0.432 & 0.433 & 0.429 & 0.428 & 0.435 & 0.436 & 0.450 & 0.450 & 0.491 & 0.492 \\
0.60 & 0.506 & 0.507 & 0.520 & 0.520 & 0.539 & 0.539 & 0.562 & 0.563 & 0.595 & 0.594 \\
0.70 & 0.593 & 0.593 & 0.623 & 0.624 & 0.650 & 0.650 & 0.674 & 0.675 & 0.697 & 0.697 \\
0.80 & 0.689 & 0.691 & 0.733 & 0.735 & 0.762 & 0.764 & 0.783 & 0.783 & 0.798 & 0.799 \\
0.90 & 0.793 & 0.794 & 0.847 & 0.846 & 0.873 & 0.875 & 0.888 & 0.890 & 0.898 & 0.899 \\
1.00 & 0.902 & 0.902 & 0.959 & 0.958 & 0.981 & 0.981 & 0.992 & 0.992 & 0.999 & 0.999 \\
1.20 & 1.127 & 1.127 & 1.177 & 1.175 & 1.189 & 1.189 & 1.195 & 1.195 & 1.199 & 1.200 \\
1.40 & 1.350 & 1.349 & 1.386 & 1.387 & 1.393 & 1.394 & 1.397 & 1.396 & 1.400 & 1.400 \\
1.50 & 1.460 & 1.459 & 1.489 & 1.490 & 1.495 & 1.495 & 1.497 & 1.496 & 1.499 & 1.500 \\
1.75 & 1.727 & 1.726 & 1.743 & 1.742 & 1.746 & 1.746 & 1.748 & 1.751 & 1.749 & 1.750 \\
2.00 & 1.985 & 1.986 & 1.996 & 1.996 & 1.997 & 1.997 & 1.999 & 1.998 & 2.000 & 1.999 \\
2.50 & 2.495 & 2.493 & 2.498 & 2.497 & 2.499 & 2.498 & 2.499 & 2.500 & 2.500 & 2.500 \\
3.00 & 2.997 & 2.996 & 2.999 & 2.999 & 2.999 & 3.000 & 3.000 & 2.999 & 3.000 & 3.000 \\
\hline & & & & & & & & & &
\end{tabular}

Table 3.7: $\left\langle\tilde{A}_{j, 1, n}\right\rangle_{R}$ values for selected $n \times$ NEX combinations and signal values $A$ ranging from 0 to 3 . For all simulations $\sigma_{g}$ was set to 1 . 


\subsection{RB Correction Sensitivity to Uncertainties in $\sigma_{g}$}

The noise variance has always been an important parameter to account for when processing and analyzing the SNR of MR images. All of the methods for magnitude correction that have been described to this point rely on accurate characterization of the noise. Ideally, $\sigma_{g}$ should be determined from the measured standard deviation of the actual ROI under consideration. However, this would, in general, involve making many measurements of the signal intensity for the ROI and calculating the standard deviation from this data set. This is impractical. An easier way to evaluate $\sigma_{g}$ is to calculate it for a large ROI located in the background at a location where $A$ can reasonably be expected to be zero and assume that the noise is the same in the ROI of interest. This has previously been shown to be a valid assumption for the scanner used [163] for all of the experiments reported in this thesis. However, obtaining an accurate estimation of $\sigma_{g}$ from the image background is not guaranteed due to the possible presence of artifacts such as flow artifacts or Gibb's ringing artifacts.

In order to assess the sensitivity of the RB correction to inaccuracies in $\sigma_{g}$ simulations were performed as a function of the amount of error in the value of $\sigma_{g}$ used. When the theoretical value of $\mu_{R}$, calculated from Eq. (3.17) was used instead of $\left\langle M_{j}\right\rangle_{R n}$ for these simulations the results were found to be very insensitive to uncertainties in $\sigma_{g}$ as large as $10 \%$. In practice, the theoretical value of $\mu_{R}$ is not known since the true $A$ value changes from pixel to pixel. The sensitivity of the RB correction to inaccuracies in $\sigma_{g}$ was also studies using a 9point nearest neighbor average as an approximation of $\mu_{R}$ in the correction term for various amounts of error in $\sigma_{g}$. These results are shown in Table 3.8. 


\begin{tabular}{ccccccc}
\hline$A$ & $\Delta \sigma_{g}=1 \%$ & $\Delta \sigma_{g}=2 \%$ & $\Delta \sigma_{g}=3 \%$ & $\Delta \sigma_{g}=4 \%$ & $\Delta \sigma_{g}=5 \%$ & $\Delta \sigma_{g}=10 \%$ \\
\hline 0.00 & 1.08 & 2.17 & 2.99 & 3.85 & 4.89 & 10.12 \\
0.10 & 0.77 & 2.06 & 2.27 & 3.09 & 4.12 & 9.30 \\
0.20 & 1.07 & 1.65 & 2.28 & 2.77 & 3.57 & 6.83 \\
0.50 & 0.12 & 0.15 & 2.06 & 4.58 & 3.67 & 1.61 \\
0.75 & 0.13 & 0.34 & 0.49 & 0.78 & 2.94 & 1.90 \\
1.00 & 0.05 & 0.11 & 0.17 & 0.28 & 0.30 & 0.84 \\
1.25 & 0.06 & 0.08 & 0.09 & 0.18 & 0.16 & 0.42 \\
1.50 & 0.11 & 0.11 & 0.12 & 0.10 & 0.13 & 0.27 \\
2.00 & 0.09 & 0.04 & 0.05 & 0.09 & 0.03 & 0.03 \\
\hline
\end{tabular}

Table 3.8: Percent error for $\left\langle\tilde{A}_{j, 19}\right\rangle_{R}$ for various amounts of error in $\sigma_{g}$ for $A$ values that ranged from 0 to 2 .

For SNR $>1$, the percent error is less than $1 \%$. However, this deviation is more pronounced when SNR decreases and a significant trend is apparent. For example, for $A=0$, a $10 \%$ error in $\sigma_{g}$ causes a $10 \%$ error in the value of $\left\langle\tilde{A}_{j, 19}\right\rangle_{R}$ when compared with the value obtained using the correct $\sigma_{g}$ value.

\subsection{Validation of the Rician Bias Correction Using a Water Phantom}

RB can be avoided by limiting the acquisition to low $b$-values where the SNR is relatively high. However, this common practice of considering only high SNR points is not valid for situations where the diffusion decay is, for example, multi-exponential since this ignores true deviations from exponentiality. With biological tissues, where diffusion is a much 
more complex phenomenon, the interpretation of diffusion decays can lead to inconsistent results due to the apparent effects introduced by the RB which can affect the values obtained for the diffusion coefficients, for multi-exponential decays, even when the signal intensity is significantly greater than the noise floor. It is, therefore, important to be able to measure the diffusion decay accurately, without RB effects, over a large range of $b$-values in order to be able to interpret tissue diffusion decays properly.

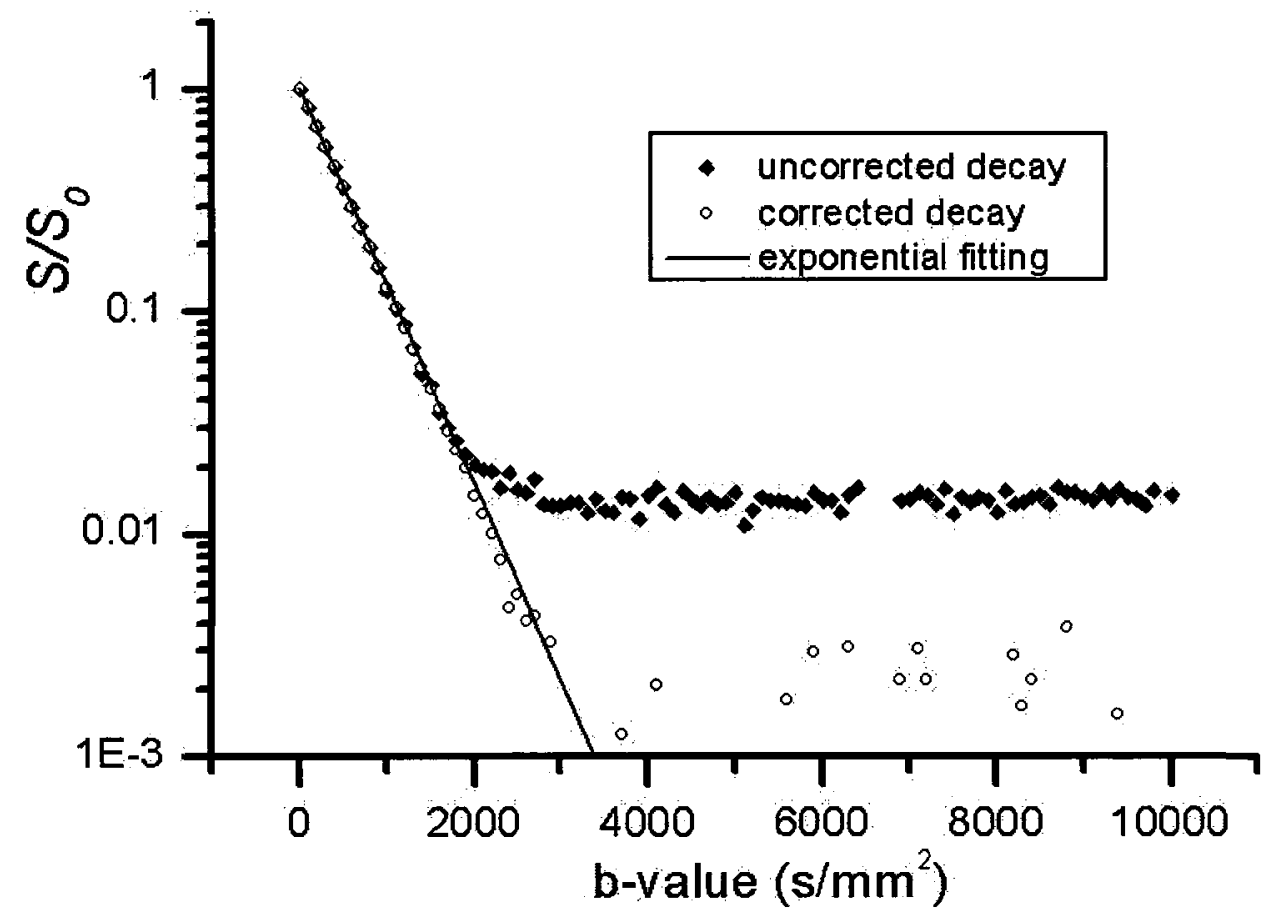

Figure 3.6: Semi-logarithmic plot of normalized diffusion decay curves for a water phantom before and after RB correction clearly showing the effect of Rician bias on the quickly decaying signals. Note the departure from exponentiality (solid line) due to the effect of Rician bias. 
A good model system for investigating the effect of Rician bias experimentally is the water diffusion decay over an extended range of $b$-values. The diffusion decay is expected to be exponential with a well known decay constant; however, when it is measured with conventional DWI it is distinctly non-exponential (see Fig. 3.6). Note the nonlinear dependence of the log of the signal intensity versus the diffusion weighting. This is solely a result of RB effects, which can be seen, for the decay shown in Fig. 3.6, when the signal drops below about $2 \%$ of its initial intensity and for $b$-values greater than about $2000 \mathrm{~s} / \mathrm{mm}^{2}$. The RB makes the water diffusion decay appear biexponential when clearly it is not. The measured diffusion decay for water should approach zero exponentially but instead it decays to an asymptotic non-zero constant value. For SNR $>3$ this effect is negligible but it is significant when $0<\mathrm{SNR}<3$. The positive bias superimposed on the diffusion decay makes it impossible to obtain an accurate fit, especially for low SNR images. The experimental details for these measurements are presented in section 4.3 .

Fig. 3.7 shows an uncorrected diffusion decay for a water phantom $\left(T E=200 \mathrm{~ms}\right.$ and $t_{d}$ $=47 \mathrm{~ms}$ ) and two RB corrected decays calculated using nearest neighbor pixel averages over one layer (9-pixels) and for several layers (225 pixels) plotted as a semi-logarithmic plot of SNR vs. $b$-value. As expected, the correction improves considerably when more pixels are used in the average. The SNR of the noise floor for the uncorrected decay is 1.25 (see dashed line) which corresponds to the mean of the Rician distribution for $A=0$, as predicted by Eq. (3.19). The noise floor for the two corrected decays is considerably lower in both cases and almost completely removed for $n=225$. When $n=9$, the value of the residual noise floor is 0.11 , and when $n=225$ it is 0.02 . However, the use of such a large number for $n$ is not always 
practical since, pixels corresponding to different tissues should not be included in the same average.

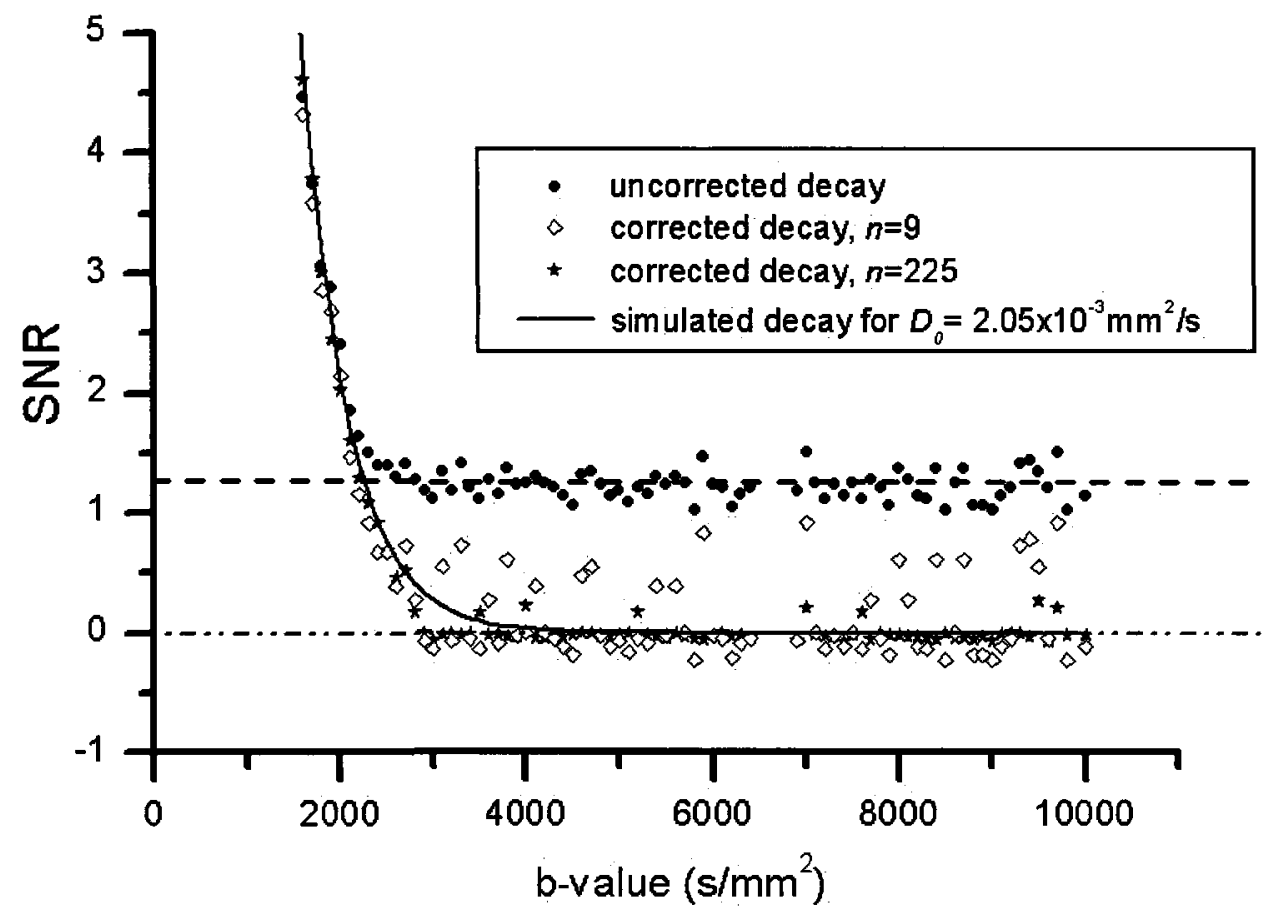

Figure 3.7: Corrected and uncorrected diffusion decay curves for a water phantom plotted as SNR vs. $b$-value. The corrected decays were calculated using 9 and 225 nearest neighbor averages. As $n$ increases, the shape of the corrected decay converges better to 0 at high bvalues. Also note the reduced spread of the corrected values when $n$ is increased. The noisy data points give an indication of the noise behavior across the full range of $b$-values, up to $10,000 \mathrm{~s} / \mathrm{mm}^{2}$. The solid line represents the expected curve for water diffusion at room temperature.

The mean slope extracted from both corrected decays was $D_{0}=(2.05 \pm 0.02) \times 10^{-3}$ $\mathrm{mm}^{2} / \mathrm{s}$ (at $20.4{ }^{\circ} \mathrm{C}$ ) which is in very good agreement with the values reported in the literature for the diffusion coefficient of pure water at room temperature [5]. 


\subsection{Discussion of the RB Correction}

The quality of information obtained from MR diffusion signal decays generated from magnitude MR images depends on many factors, including equipment, acquisition parameters, and post-processing methods. Much work has been done to minimize the effects of Rician bias on the diffusion signal decays for heterogeneous systems like tissue. The Rician bias shows its most dominant effect on the decay when the SNR is low which occurs when the diffusivity is high or when large $b$-values are used. RB effects cause incorrect values to be obtained for fit parameters such as the diffusion coefficients and their fractional contribution to the decay from the analysis of these decay curves.

This chapter had examined in detail the implementation of the theory developed by Koay and Basser [159] which can simultaneously determine the unbiased PDF mean and variance from magnitude images when the Rician mean and standard deviation are known exactly and can be used to compensate for the effects of Rician bias at low SNR. A new implementation of a RB correction technique using a convergence criterion for the binomial expansion was also shown to be far superior to retaining only a small number of terms in the expansion for small SNR values. This new procedure was validated using both simulations and experiments performed on a water phantom.

All of the methods for RB correction that have been discussed here rely on accurate characterization of the noise and the assumption that the noise level is the same for all voxels in the image set. Simulations results have shown that the RB correction is relatively insensitive to errors in $\sigma_{g}$ and that the value of $\sigma_{g}$ obtained from the image background does not change as NEX increases. 
The RB correction method used here was primarily intended for signal averaged images. It was shown that, even though the shape of the PDF changes as NEX increases, the PDF mean does not change. Since the correction scheme employed here is derived from the equation for the Rician PDF mean $(\mathrm{NEX}=1)$ it is clear that Rician bias correction is equally valid for data sets with NEX $>1$ since the PDF mean is unaffected by the signal averaging.

\subsection{Conclusions}

This chapter dealt in detail with the estimation of RB from MRI images with special reference to magnitude averaged MR images. The significant step made by Koay and Basser of presenting an analytically exact correction scheme is very important but it is often not practical to use their approach in practice, especially for most situations of interest in vivo, since their approach requires accurate values for both the mean and standard deviation of the Rician PDF. This necessitates multiple acquisitions in order to get reliable values. However, by using a binomial expansion of their expression, employing a convergence criterion and introducing nearest neighbor averaging, the new correction scheme described here provides very good RB correction with minimal PDF distortion for both NEX $=1$ and NEX $>1$. 


\section{Chapter 4}

\section{Experimental Methods}

\subsection{MR Experimental Protocols}

\subsection{1 $\mathrm{T}_{2}$ Measurements}

$T_{2}$ decays have traditionally been measured using multi-echo data sets acquired during a spin echo readout train. The sequence used to perform the experiments reported here was a Siemens sequence called se_mc, which is a linearly sampled 32-echo MRI pulse sequence. All $T_{2}$ measurements were performed on the Siemens Symphony TIM 1.5 T MR scanner at the General Campus of The Ottawa Hospital using the 16-channel phased array head coil. The scanner was operating with Syngo B15 software. The se_mc sequence used for these measurements is a Carr-Purcell-Meiboom-Gill (CPMG) sequence which consists of a sliceselective $90^{\circ}$ pulse followed by multiple slice-selective $180^{\circ}$ refocusing pulses which generate 
an echo train. For this sequence the echo times for the echoes in the train are integer multiples of the initial echo time. In vivo sequence parameters were: $T R=6 \mathrm{~s}$, number of slices $=1$, slice thickness $=5 \mathrm{~mm}$, the field of view was $30 \times 30 \mathrm{~cm}^{2}$ and the image matrix resolution was $128 \times 128$. No signal averaging was used. The total scan time was 15 minutes. In order to obtain reliable $T_{2}$ values a sufficient range of echo times must be sampled. Furthermore, criteria for in vivo human brain studies suggest that the initial echo spacing should be as short as feasible (10 ms or less) and that the echo train length should be such that the last echoes contain only noise. $T_{2}$ decay measurements are not routinely acquired in a clinical environment because of these constraints and the time involved with making such measurements. To minimize the initial echo time and at the same time to elongate the range of echo times sampled, 3 different data sets with echo spacings of $8.1,9.1$ and $10 \mathrm{~ms}$ were combined together resulting in a 96-echo $T_{2}$ decay, non-linearly sampled over the range $8.1 \mathrm{~ms}$ to 320 ms. Additionally, two 32-echo data sets were measured independently where the linear echo spacing was changed from $8.1 \mathrm{~ms}$ to $16.2 \mathrm{~ms}$. All other experimental parameters were kept the same. The maximum $T E$ for these decays was $518.4 \mathrm{~ms}$.

\subsubsection{Diffusion Measurements}

All diffusion measurements were performed on the same clinical scanner as mentioned in the previous section; however, they were performed before the scanner was upgraded to a TIM system and from A25a to B15 Syngo software. A CP head coil was used for the diffusion experiments.

The scanning protocol consisted of a localizer, 6 sets of diffusion weighted sequences with 16 different $b$-values each and a DTI sequence. The diffusion-weighted images were 
acquired using Siemens diffusion-weighted single-shot EPI sequence which is designed to minimize the effects of eddy currents induced by the diffusion gradients. The 96 b-values used were chosen between 0 and $10,000 \mathrm{~s} / \mathrm{mm}^{2}$. The matrix size of $128 \mathrm{x} 96$ was interpolated to $128 \times 128$, the field of view was $30 \times 30 \mathrm{~cm}^{2}$, the slice thickness was $5 \mathrm{~mm}$ and the voxel size was $2.34 \times 2.34 \times 5 \mathrm{~mm}^{3}$. Other parameters were: $T R / T E=930 / 200 \mathrm{~ms}, \Delta / \delta=74 / 68 \mathrm{~ms}$ and 6 averages were used. The diffusion sensitizing gradients were rotated in the $x-z$ plane from $\alpha=$ $0^{\circ}$ (i.e. parallel to the $x$-axis) to $90^{\circ}$ in increments of $15^{\circ}$. The frequency encoding and slice selection directions were across the bore of the magnet (i.e. the $x$-direction) and along the bore (i.e. the $z$-direction), respectively. The whole set of 96 diffusion-weighted images was acquired in $9 \min 18 \mathrm{~s}$ for 3 slices for each diffusion gradient orientation.

To assess the diffusion tensor, a second diffusion experiment was performed along 12 non-collinear gradient directions with $b=0$ and $500 \mathrm{~s} / \mathrm{mm}^{2}, T R / T E=4300 / 117 \mathrm{~ms}$ and 2 averages were used. The other sequence parameters, including slice location and thickness, were the same as for the DWI measurements described above. The DTI protocol took less than 2 minutes.

\subsubsection{Subjects and Slice Location}

Two healthy male volunteers, aged 51 and 36 years old, were recruited. The project was approved by the Ottawa Hospital Research Ethics Board and informed consent was received from both subjects. The same two subjects were used for both the $T_{2}$ and diffusion studies.

The slice positioning protocol was carefully developed to ensure that the same slice was imaged in each session. For the acquisition of the $T_{2}$ and diffusion-weighted images, the 
slice of interest (SI) was always positioned superior to, and parallel with, a central slice (SC) with a $10 \mathrm{~mm}$ gap between the slices. The SC was a reference slice located along the inferior border of the corpus callosum (identified from a true mid-sagittal view, see Fig. 4.1) which was used as a reference landmark. The images were oblique slices oriented approximately $15^{\circ}$ towards coronal from transverse. The images were inspected for right-left brain symmetry to ensure that the subject's head was not tilted and that the right-left white matter tracts were oriented parallel to the $x$-axis of the scanner so that diffusion sensitizing gradients applied along the $x$ and $y$ axes of the scanner were indeed parallel and perpendicular, respectively, to the predominant fibre orientation in the voxels of interest.

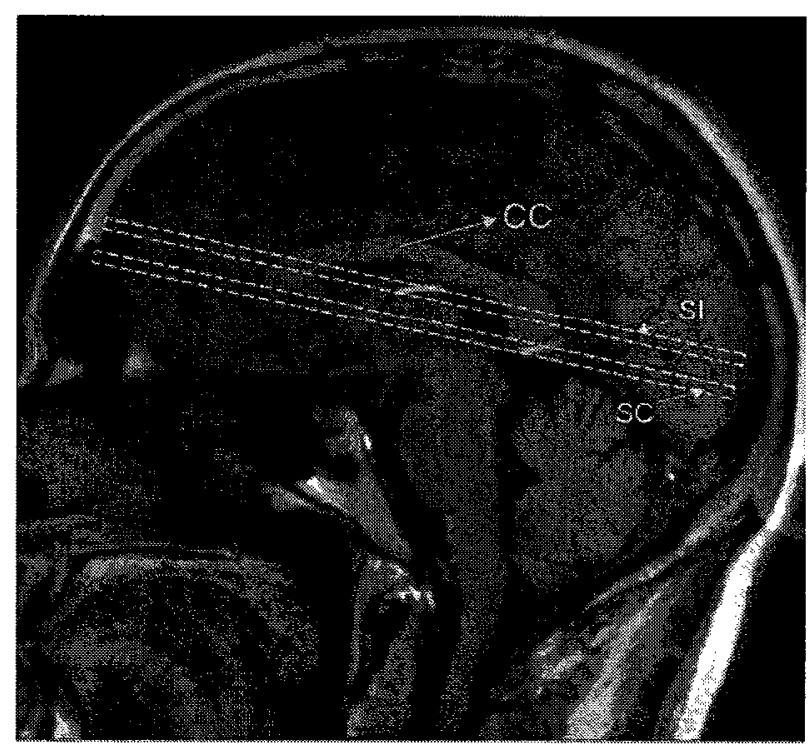

Figure 4.1: Sagittal view showing the slice of interest (SI) oriented parallel to the reference central slice (SC). The compact corpus callosum (CC) structure is well seen in this sagittal plane.

Another important aspect of the experimental design was the selection of brain regions for which the cell geometry was as well known and as uniform as possible. The $T_{2}$ relaxation and diffusion decay data were averaged over 9 and 6 pixel ROIs located in the splenium of the 
corpus callosum (SCC) and genu of the corpus callosum (GCC), respectively (see Fig. 4.2). The corpus callosum (CC) is the most distinguishable structure of the central nervous system on DTI. The CC fiber distribution is known to be compact with larger myelinated fibers populating the central and posterior portions whereas the anterior aspect contains a large number of smaller fibers. The SCC and GCC were chosen for study since they have high fractional anisotropy $(F A)$ values, as determined from the DTI measurements. This ensures that there is good uniformity in fibre orientation for these voxels. Relatively small ROIs were chosen to keep the $F A$ values as uniform as possible across the ROI. The predominant axonal orientation is known to be left to right in both the SCC and the GCC.

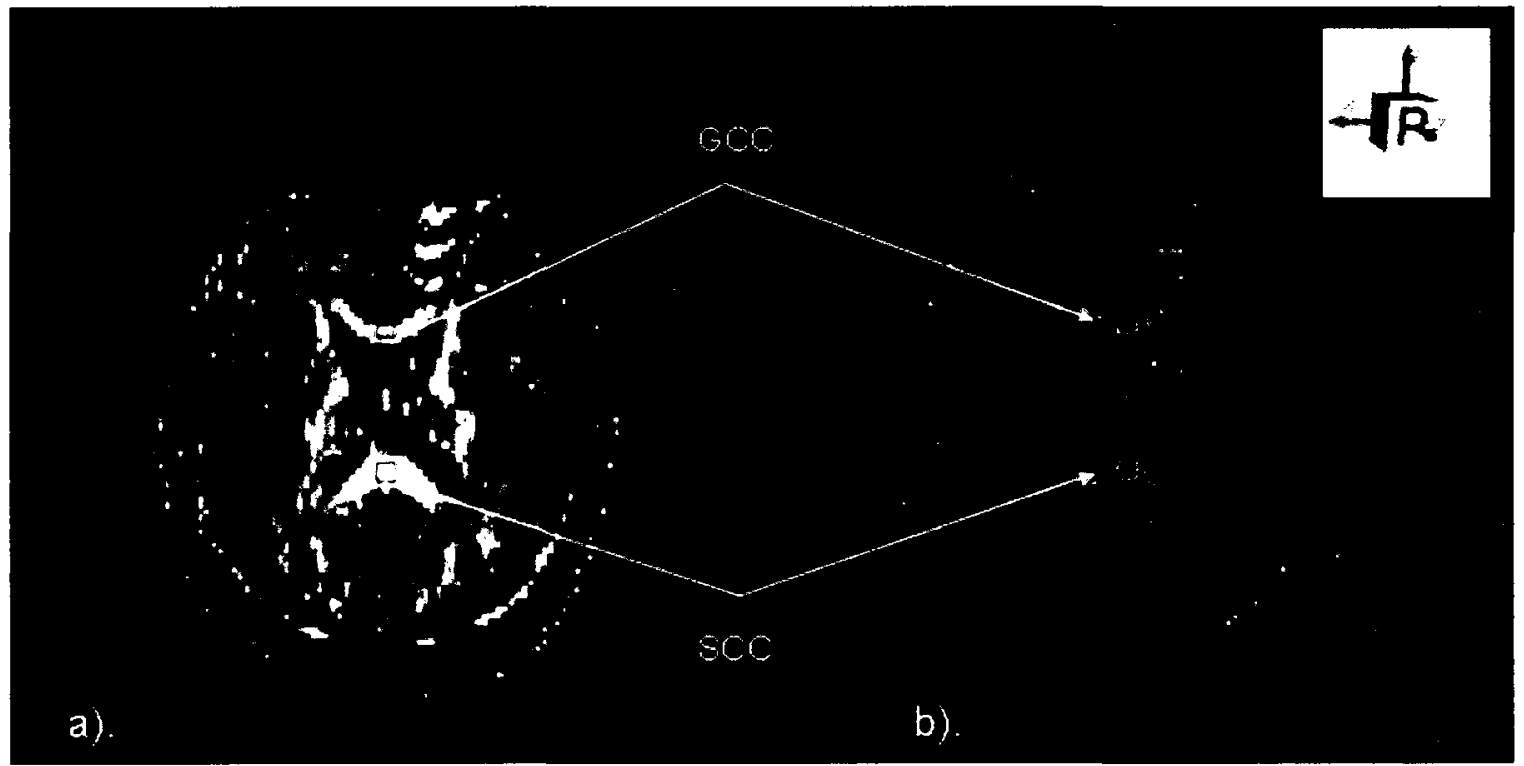

Figure 4.2: a). Axial $F A$ maps showing the degree of anisotropy in each voxel. In white matter, where anisotropy is high, the pixels appear bright on a grey scale. b). A color-coded white matter fiber map generated on the basis of fractional anisotropy and eigenvector information. Red, green and blue pixels indicate axonal fibers oriented in the left-right, anterior-posterior and inferior-superior directions, respectively. The ROIs were selected within the SCC and GCC, as shown. 
With this ROI selection, it could be reasonably certain that the axons in the voxels being studied are oriented predominantly in the same direction and that direction is parallel to the frequency encoding direction in the DWI images. The experiments were designed to measure diffusion parallel and perpendicular to the axons by setting the diffusion sensitization in the left-right (LR) and inferior-superior (IS) directions, respectively, which correspond to the frequency encoding and slice selection directions, respectively.

\subsection{Decay Curve Analysis}

The $T_{2}$ analysis reported here identifies the relaxation components present in a measured decay curve based on an assumed three component model. The majority of relaxation studies in the literature also report three components for human white matter [165167].

The resulting signal decay curves were fit to a sum of exponential functions of the following form:

$$
S=S_{0} \sum_{i=1}^{N_{c}} f_{i} \exp \left(-T E / T_{2}(i)\right)
$$

where $S_{0}$ is the signal strength at $T E=0, f_{i}$ is the signal fraction for component $i, T_{2}(i)$ is the corresponding relaxation time and $N_{c}$ is the number of exponential components being considered.

The diffusion coefficients and their signal fractions were determined by fitting the diffusion decay curves to a biexponential function of the form:

$$
S / S_{0}=f_{\text {fast }} \exp \left(-b^{*} D_{\text {fast }}\right)+f_{\text {slow }} \exp \left(-b^{*} D_{\text {slow }}\right)
$$


where $S_{0}$ is the signal strength for $b=0, f_{\text {fast }}$ and $f_{\text {slow }}$ are the signal fractions of the fast and slow diffusion components $\left(f_{\text {fast }}+f_{\text {slow }}=1\right)$, with diffusion coefficients of $D_{\text {fast }}$ and $D_{\text {slow }}$, respectively.

The fitting routines used to analyze both the multicomponent $T_{2}$ relaxation and diffusion data were performed based on the Levenberg-Marquardt non-linear least squares algorithm [168] provided by Origin (Microcal Software Inc.). This software package also provided the coefficient of determination $\left(R^{2}\right)$ as a global statistical measure of the goodness of the fit. A good fit is indicated by values of $R^{2}$ close to 1 . Mathematically, $R^{2}$ is defined by the equation:

$$
R^{2}=1-\frac{S S_{e r r}}{S S_{t o t}}
$$

where $S S_{\text {err }}$ is sum of squares of the differences between the data points and the values predicted for the same abscissa by the functional fit and $S S_{\text {tot }}$ is the total variance of the data [169].

\subsection{Water Phantom Measurements}

The noise bias correction technique discussed in Chapter 3 is based on the theoretical prediction that the data distribution is Rician and the availability of an accurate value for $\sigma_{g}$. To test this post-processing scheme, a series of experiments using a water phantom where performed.

The Siemens head coil was used to measure the diffusion signals $S(b)$ for $96 b$-values ranging from 0 to $10,000 \mathrm{~s} / \mathrm{mm}^{2}$. A single transverse (i.e. the head to foot direction) $5 \mathrm{~mm}$ thick slice was selected. The phase encoding direction was chosen to be in the anterior to 
posterior direction while the diffusion sensitizing gradients was applied in the right to left direction. The measurements were performed with a 128 matrix and a $75 \%$ partial Fourier factor. Thus, the data was acquired as a $128 \times 96$ matrix in $k$-space and reconstructed as $128 \times 128$ images. Other imaging parameters were: $\mathrm{FOV}=30 \times 30 \mathrm{~cm}^{2}, T R / T E=930 / 200 \mathrm{~ms}$, voxel size was $2.34 \times 2.34 \times 5 \mathrm{~mm}^{3}$ and $\Delta / \delta=74 / 68 \mathrm{~ms}$. The diffusion decay signal was averaged 6 times (i.e. NEX=6).

Two different sized regions were selected, a 9-pixel ROI and a 225-pixel ROI. The signal was reported as the average over the pixels in each ROI. A 300-pixel ROI was drawn in the image background to compute $\sigma_{g}$ based on Eq. (3.19). 


\section{Chapter 5}

\section{Results}

\section{$5.1 \mathrm{~T}_{2}$ Measurements}

In this section, results of $T_{2}$ decay measurements are presented for the splenium of the corpus callosum (SCC) and the genu of the corpus callosum (GCC) for two healthy male subjects. Figure 5.1 shows a semi-logarithmic plot of a typical $T_{2}$ relaxation decay for the SCC; the curves for the GCC are not shown but they are very similar. The nonlinear shape demonstrates that this decay is multicomponent, as expected for inhomogeneous, compartmentalized white matter systems. The noise floor values extracted from the background for each corresponding $T E$ are also shown.

The $T_{2}$ curves were fitted assuming a tri-exponential function since there seems to be a consensus in the literature that there are three $T_{2}$ in white matter. This is discussed further in Section 6.1.1. The curve fitting provided values for the three different $T_{2}$ relaxation times and 
their corresponding signal fractions, $f_{1}, f_{2}$ and $f_{3}$, expressed as a fraction of the total signal magnitude extrapolated to $T E=0$. No significant differences were observed for the water relaxation times between the subjects for either the SCC or the GCC. Tables 5.1 and 5.2 summarize all fit parameter values for the 51 year old and the 36 year old subjects, respectively. The mean and standard deviation (SD) over the 7 trials for each subject along with the fractional anisotropy $(F A)$ for each trial are also given. The $T_{2}$ decay curves were sampled over the large $T E$ range of $16.2 \mathrm{~ms}$ to $340 \mathrm{~ms}$. None of these decays differ in any significant way from the decay presented in Fig. 5.1. The MR pulse sequence did not introduce significant Rician bias into the $T_{2}$ relaxation decay curve since the signal intensity was well above the noise floor for all $T E$ values. None of these decays required RB correction.

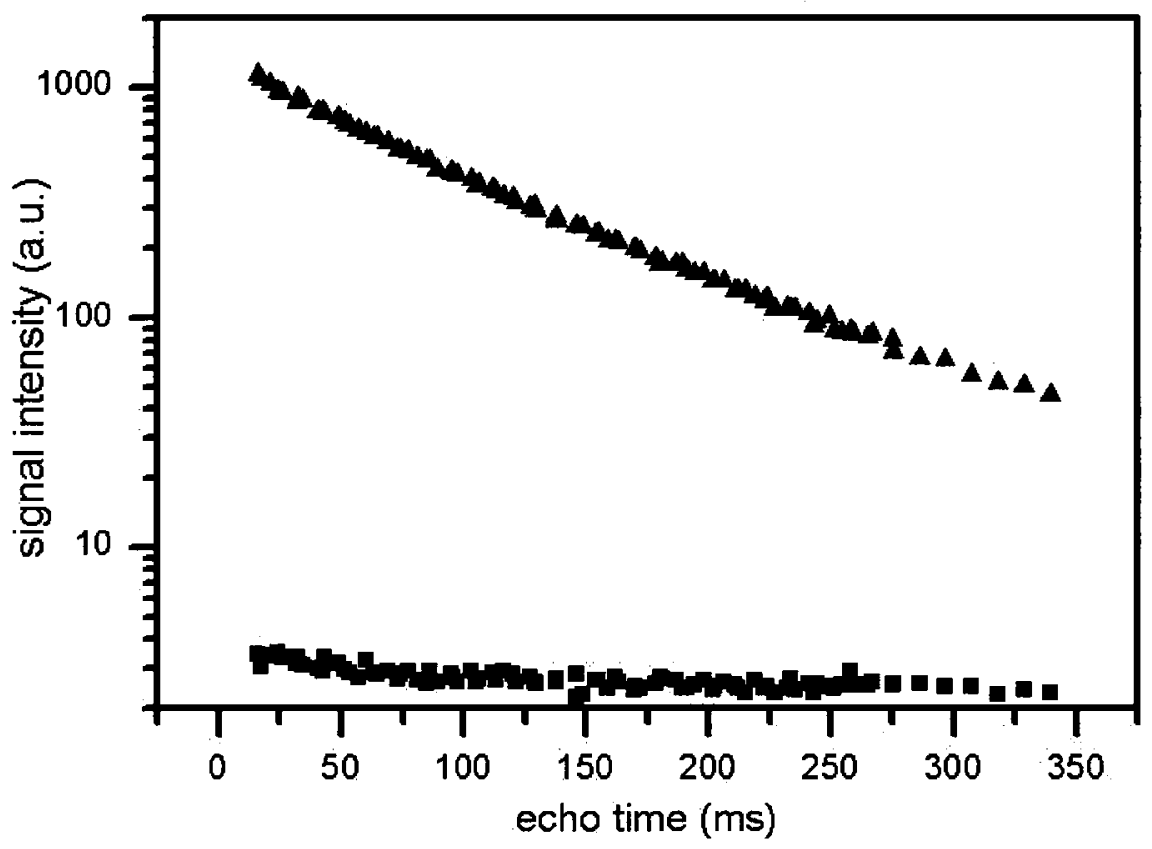

Figure 5.1: A typical $T_{2}$-decay curve for the SCC (triangles) along with the baseline noise (squares). Note that the signal intensity, expressed in arbitrary units (a.u.), remains well above baseline noise values for all echoes. 


\begin{tabular}{|ccccccccc|}
\hline & $f_{1}$ & $T_{2}(1)$ & $f_{2}$ & $T_{2}(2)$ & $f_{3}$ & $T_{2}(3)$ & $R^{2}$ & $F A$ \\
\hline trial1 & 0.11 & $17 \pm 6$ & 0.78 & $80 \pm 19$ & 0.11 & $224 \pm$ & 0.9992 & 0.83 \\
& \pm 0.02 & & \pm 0.07 & & \pm 0.04 & 161 & & \\
trial2 & 0.11 & $15 \pm 4$ & 0.69 & $73 \pm 17$ & 0.21 & $167 \pm 87$ & 0.9995 & 0.85 \\
& \pm 0.02 & & \pm 0.07 & & \pm 0.04 & & & \\
trial3 & 0.07 & $16 \pm 6$ & 0.74 & $75 \pm 3$ & 0.19 & $179 \pm 22$ & 0.9993 & 0.88 \\
& \pm 0.02 & & \pm 0.01 & & \pm 0.02 & & & \\
trial4 & 0.09 & $14 \pm 6$ & 0.65 & $69 \pm 19$ & 0.27 & $148 \pm 62$ & 0.9995 & 0.87 \\
& \pm 0.02 & & \pm 0.03 & & \pm 0.03 & & & \\
trial5 & 0.11 & $17 \pm 4$ & 0.73 & $76 \pm 12$ & 0.16 & $198 \pm 33$ & 0.9994 & 0.85 \\
& \pm 0.01 & & \pm 0.10 & & \pm 0.03 & & & \\
trial6 & 0.10 & $17 \pm 4$ & 0.79 & $78 \pm 7$ & 0.11 & $228 \pm 73$ & 0.9995 & 0.83 \\
& \pm 0.03 & & \pm 0.05 & & \pm 0.07 & & & \\
trial7 & 0.09 & $16 \pm 3$ & 0.79 & $77 \pm 7$ & 0.11 & $229 \pm 36$ & 0.9994 & 0.83 \\
& \pm 0.01 & & \pm 0.04 & & \pm 0.02 & & & \\
mean & $\mathbf{0 . 1 0}$ & $\mathbf{1 6} \pm \mathbf{1}$ & $\mathbf{0 . 7 4}$ & $\mathbf{7 6} \pm \mathbf{4}$ & $\mathbf{0 . 1 7}$ & $\mathbf{1 9 6} \pm \mathbf{3 3}$ & & $\mathbf{0 . 8 5}$ \\
$\pm \mathbf{S D}$ & $\mathbf{0 . 0 1}$ & & $\mathbf{0 . 0 5}$ & & $\pm \mathbf{0 . 0 6}$ & & & $\mathbf{0 . 0 2}$ \\
\hline
\end{tabular}

a).

\begin{tabular}{|ccccccccc|}
\hline & $f_{1}$ & $T_{2}(1)$ & $f_{2}$ & $T_{2}(2)$ & $f_{3}$ & $T_{2}(3)$ & $R^{2}$ & $F A$ \\
\hline trial1 & 0.17 & $16 \pm 2$ & 0.71 & $85 \pm 10$ & 0.12 & $419 \pm 297$ & 0.9994 & 0.87 \\
& \pm 0.03 & & \pm 0.06 & & \pm 0.08 & & & \\
trial2 & 0.09 & $13 \pm 6$ & 0.77 & $77 \pm 3$ & 0.14 & $442 \pm 262$ & 0.9976 & 0.85 \\
& \pm 0.03 & & \pm 0.02 & & \pm 0.09 & & & \\
trial3 & 0.12 & $12 \pm 2$ & 0.64 & $89 \pm 3$ & 0.24 & $528 \pm 17$ & 0.9989 & 0.88 \\
& \pm 0.01 & & \pm 0.01 & & \pm 0.02 & & & \\
trial4 & 0.14 & $13 \pm 2$ & 0.72 & $89 \pm 3$ & 0.14 & $386 \pm 86$ & 0.9987 & 0.87 \\
& \pm 0.02 & & \pm 0.01 & & \pm 0.03 & & & \\
trial5 & 0.11 & $11 \pm 4$ & 0.61 & $76 \pm 16$ & 0.27 & $236 \pm 86$ & 0.9988 & 0.85 \\
& \pm 0.03 & & \pm 0.14 & & \pm 0.16 & & & \\
trial6 & 0.19 & $14 \pm 2$ & 0.73 & $91 \pm 3$ & 0.08 & $278 \pm 21$ & 0.9990 & 0.88 \\
& \pm 0.02 & & \pm 0.01 & & \pm 0.01 & & & \\
trial7 & 0.20 & $13 \pm 1$ & 0.71 & $89 \pm 2$ & 0.09 & $256 \pm 17$ & 0.9988 & 0.84 \\
& \pm 0.02 & & \pm 0.01 & & \pm 0.01 & & & \\
mean & $\mathbf{0 . 1 5}$ & $\mathbf{1 3} \pm \mathbf{2}$ & $\mathbf{0 . 6 9}$ & $\mathbf{8 5} \pm \mathbf{6}$ & $\mathbf{0 . 1 5}$ & $\mathbf{3 6 4} \pm \mathbf{1 0 9}$ & & $\mathbf{0 . 8 6}$ \\
$\mathbf{E D}$ & $\mathbf{0 . 0 4}$ & & $\mathbf{0 . 0 5}$ & & $\mathbf{0 . 0 7}$ & & & $\pm \mathbf{0 . 0 2}$ \\
\hline
\end{tabular}

b).

Table 5.1: Signal fraction, $f_{i}$, and $T_{2}(i)$ transverse relaxation time estimates associated with each water fraction, $i$, for (a) the SCC and (b) the GCC of the 51 year old volunteer. The mean values plus and minus their standard deviations (SD) are also included for each parameter. 


\begin{tabular}{|c|c|c|c|c|c|c|c|c|}
\hline & $f_{l}$ & $T_{2}(1)$ & $f_{2}$ & $T_{2}(2)$ & $f_{3}$ & $T_{2}(3)$ & $R^{2}$ & $F A$ \\
\hline trial1 & $\begin{array}{c}0.07 \\
\pm 0.06\end{array}$ & $21 \pm 11$ & $\begin{array}{c}0.62 \\
\pm 0.06\end{array}$ & $61 \pm 20$ & $\begin{array}{c}0.32 \\
\pm 0.11\end{array}$ & $156 \pm 42$ & 0.9996 & 0.91 \\
\hline trial2 & $\begin{array}{c}0.13 \\
\pm 0.02\end{array}$ & $15 \pm 3$ & $\begin{array}{c}0.71 \\
\pm 0.06\end{array}$ & $73 \pm 11$ & $\begin{array}{c}0.16 \\
\pm 0.10\end{array}$ & $205 \pm 91$ & 0.9996 & 0.91 \\
\hline trial3 & $\begin{array}{c}0.09 \\
\pm 0.04\end{array}$ & $17 \pm 11$ & $\begin{array}{c}0.69 \\
\pm 0.06\end{array}$ & $75 \pm 8$ & $\begin{array}{c}0.23 \\
\pm 0.08\end{array}$ & $186 \pm 21$ & 0.9994 & 0.89 \\
\hline trial4 & $\begin{array}{c}0.12 \\
\pm 0.04\end{array}$ & $17 \pm 6$ & $\begin{array}{c}0.62 \\
\pm 0.05\end{array}$ & $71 \pm 30$ & $\begin{array}{c}0.26 \\
\pm 0.04\end{array}$ & $141 \pm 89$ & 0.9995 & 0.91 \\
\hline trial5 & $\begin{array}{c}0.12 \\
\pm 0.04\end{array}$ & $19 \pm 6$ & $\begin{array}{c}0.67 \\
\pm 0.10\end{array}$ & $72 \pm 20$ & $\begin{array}{c}0.21 \\
\pm 0.08\end{array}$ & $174 \pm 93$ & 0.9996 & 0.95 \\
\hline trial6 & $\begin{array}{c}0.09 \\
\pm 0.04\end{array}$ & $16 \pm 4$ & $\begin{array}{c}0.65 \\
\pm 0.03\end{array}$ & $69 \pm 4$ & $\begin{array}{c}0.26 \\
\pm 0.06\end{array}$ & $152 \pm 41$ & 0.9992 & 0.92 \\
\hline trial7 & $\begin{array}{c}0.12 \\
\pm 0.02\end{array}$ & $22 \pm 4$ & $\begin{array}{c}0.72 \\
\pm 0.09\end{array}$ & $77 \pm 3$ & $\begin{array}{c}0.16 \\
\pm 0.02\end{array}$ & $195 \pm 33$ & 0.9992 & 0.91 \\
\hline $\begin{array}{l}\text { mean } \\
\pm S D\end{array}$ & $\begin{array}{c}0.11 \\
\pm 0.02\end{array}$ & $18 \pm 3$ & $\begin{array}{c}0.67 \\
\pm 0.04\end{array}$ & $71 \pm 5$ & $\begin{array}{c}0.23 \\
\pm 0.06\end{array}$ & $173 \pm 24$ & & $\begin{array}{r}0.91 \\
\pm 0.02\end{array}$ \\
\hline
\end{tabular}

a).

\begin{tabular}{|ccccccccc|}
\hline & $f_{1}$ & $T_{2}(1)$ & $f_{2}$ & $T_{2}(2)$ & $f_{3}$ & $T_{2}(3)$ & $R^{2}$ & $F A$ \\
\hline trial1 & 0.11 & $16 \pm 4$ & 0.76 & $73 \pm 3$ & 0.13 & $170 \pm 42$ & 0.9993 & 0.87 \\
& \pm 0.02 & & \pm 0.01 & & \pm 0.02 & & & \\
trial2 & 0.08 & $10 \pm 6$ & 0.65 & $64 \pm 5$ & 0.27 & $297 \pm 14$ & 0.9923 & 0.84 \\
& \pm 0.05 & & \pm 0.03 & & \pm 0.02 & & & \\
trial3 & 0.11 & $15 \pm 4$ & 0.68 & $65 \pm 2$ & 0.21 & $322 \pm 11$ & 0.9967 & 0.90 \\
& \pm 0.01 & & \pm 0.01 & & \pm 0.02 & & & \\
trial4 & 0.16 & $11 \pm 2$ & 0.72 & $72 \pm 9$ & 0.12 & $215 \pm 116$ & 0.9993 & 0.87 \\
& \pm 0.02 & & \pm 0.09 & & \pm 0.11 & & & \\
trial5 & 0.18 & $14 \pm 2$ & 0.79 & $80 \pm 3$ & 0.08 & $188 \pm 53$ & 0.9991 & 0.86 \\
& \pm 0.02 & & \pm 0.01 & & \pm 0.02 & & & \\
trial6 & 0.11 & $13 \pm 5$ & 0.73 & $74 \pm 12$ & 0.07 & $368 \pm 164$ & 0.9973 & 0.88 \\
& \pm 0.04 & & \pm 0.07 & & \pm 0.11 & & & \\
trial7 & 0.18 & $12 \pm 2$ & 0.74 & $75 \pm 4$ & 0.08 & $154 \pm 21$ & 0.9985 & 0.86 \\
& \pm 0.02 & & \pm 0.02 & & \pm 0.03 & & & \\
mean & $\mathbf{0 . 1 3}$ & $\mathbf{1 3} \pm \mathbf{2}$ & $\mathbf{0 . 7 2}$ & $\mathbf{7 2} \pm \mathbf{6}$ & $\mathbf{0 . 1 4}$ & $\mathbf{2 4 5} \pm \mathbf{8 4}$ & & $\mathbf{0 . 8 7}$ \\
$\mathbf{E S D}$ & $\mathbf{0 . 0 4}$ & & $\mathbf{0 . 0 5}$ & & $\mathbf{0 . 0 8}$ & & & $\mathbf{0 . 0 2}$ \\
\hline
\end{tabular}

b).

Table 5.2: Signal fraction, $f_{i}$, and $T_{2}(i)$ transverse relaxation time estimates associated with each water fraction, $i$, for (a) the SCC and (b) the GCC of the 36 year old volunteer. The mean values plus and minus their standard deviations (SD) are also included for each parameter. 
Each subject was scanned in 3 different sessions to test for intra-session and intersession variability. The acquired images contained both the SCC and the GCC. The first session consisted of 3 trials and the last 2 sessions had 2 trials each. Between trials within a given session the subject was removed from the scanner. The results for all trials are very consistent; no system systematic intra-session or inter-session variability was observed. In this manner, four sets of $T_{2}$ decays were acquired, two for each subject. Furthermore, as can be seen from Table 5.1, the results were not significantly different for the two subjects or for the two ROIs studied.

For quantitative analysis, the echo spacing should ideally be as short as feasible and the echo train length should be such that the last echoes report only noise. For the in vivo $T_{2}$ measurements reported above, the acquired images had echo times no greater than $340 \mathrm{~ms}$. Nevertheless, the fitted values display a reasonable degree of correspondence with the values obtained in most in vivo human brain studies for this $T E$ time scale. This is discussed further in section 6.1.2.

In order to assess how much the $T E$ timescale influences the $T_{2}(i)$ and the $f_{i}$, an extra set of 32 echoes with a $16.2 \mathrm{~ms}$ echo spacing (the data for the first echo was rejected) was acquired for trial 7 for the 51 year old subject. The maximum $T E$ for this decay was $518.4 \mathrm{~ms}$. This allowed us to follow the decay down to $2 \%$ of its initial signal, which is still significantly above the noise level. The fit parameters obtained from this trial with and without the extra echoes are given in Table 5.3. The long $T E$-range employed in the second experiment had virtually no effect on the fit parameter values obtained. $T_{2}$ measurements acquired with prolonged $T E$ echoes are impractical for most clinical applications and are sometimes complicated by the effects of motion during the long acquisition. 


\begin{tabular}{|cccccccc|}
\hline & $f_{1}$ & $T_{2}(1)$ & $f_{2}$ & $T_{2}(2)$ & $f_{3}$ & $T_{2}(3)$ & $R^{2}$ \\
\hline 93-echoes & 0.09 & 16 & 0.79 & 77 & 0.11 & 230 & 0.99043 \\
124-echoes & 0.10 & 18 & 0.78 & 78 & 0.12 & 224 & 0.99952 \\
\hline
\end{tabular}

Table 5.3: Signal fractions and $T_{2}$ relaxation times from trial 7 for the 51 year old subject reported in Table 5.1a with and without an extra set of echoes. The 93-echo data set was acquired using the standard protocol and the 124-echo data set included the same 93 echoes plus an additional 31 echoes which extended the decay to $518.4 \mathrm{~ms}$.

The relaxation data was collected with $T R=6 \mathrm{~s}$. The $T_{l}$ losses for these experiments should be negligible since:

$$
S_{T_{2}} \cong S_{0}\left(1-e^{-\left(T R-T E_{\max }\right) / T_{1}}\right)
$$

where $T_{1}$ is the spin-lattice relaxation time, $S_{0}$ is the initial signal at $\mathrm{t}=0, T E_{\max }$ is the largest echo time used and $S_{T 2}$ is the signal corrected for the $T_{1}$ losses. For white matter, $T_{1} \approx 800 \mathrm{~ms}$ [163].

In spite of its popularity, the standard multiple spin-echo sequence used for the experiments reported here has been found to be insufficient for accurate $T_{2}$ measurements due to residual sensitivity to refocusing imperfections. This normally results in the formation of stimulated (secondary) echoes that disrupt the $T_{2}$ decay of the primary spin echoes. Therefore, an investigation of the quantitative accuracy of the $T_{2}$ values was undertaken since it was found that the signal intensity from the first echo, independent of the echo spacing, was lower than it should be and often less than the signal intensity from the second echo (see Fig. 5.2).

Two 32 echo data sets were measured independently where the linear echo spacing was changed from $8.1 \mathrm{~ms}$ to $16.2 \mathrm{~ms}$. All other experimental parameters were kept the same. It was observed that the second signal point (corresponding to $T E=32.4 \mathrm{~ms}$ ) of the second set 

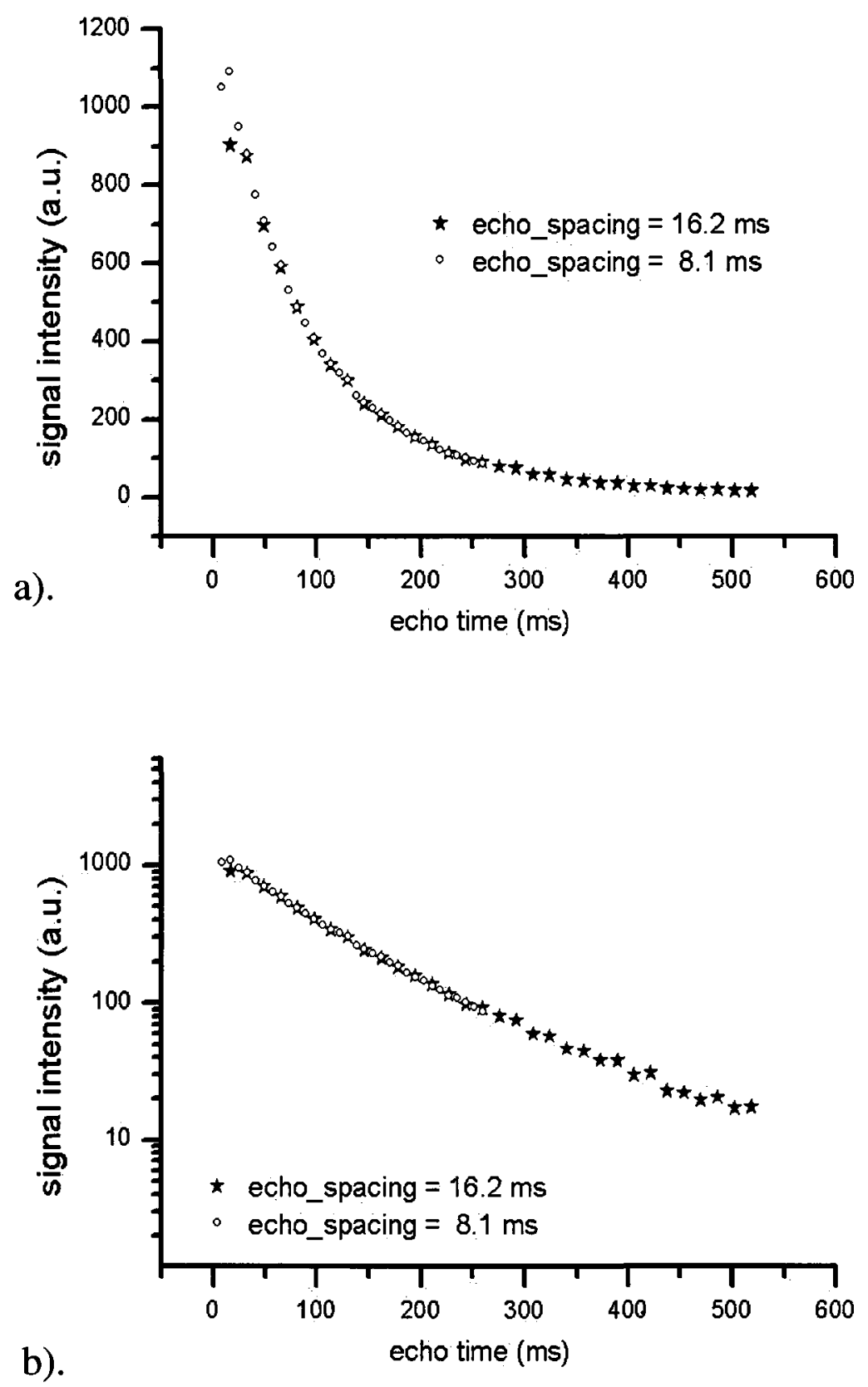

Figure 5.2: Typical $T_{2}$ decay curves consisting of signal intensity (in arbitrary units) versus echo time, $T E$, for the 51 year old subject acquired in the same imaging session. Two sets of 32 echoes are shown in the figure corresponding to echo spacings of 8.1 and $16.2 \mathrm{~ms}$. In a) the decay is shown on a linear plot and in b) the same data are shown on a semi-logarithmic plot. 
was superimposed on the fourth point of the first data set for the same TE, (see Fig. 5.2). No difference between these $T_{2}$ signal decay curves is visible, except for their first echo points which were found to be erroneously low. The experimental validity of the $T_{2}$ analysis should not be compromised by discarding the first data point since the second and later echo points does not seem to be affected. This effect is attributed to spurious magnetization from stimulated echoes $[170,171]$.

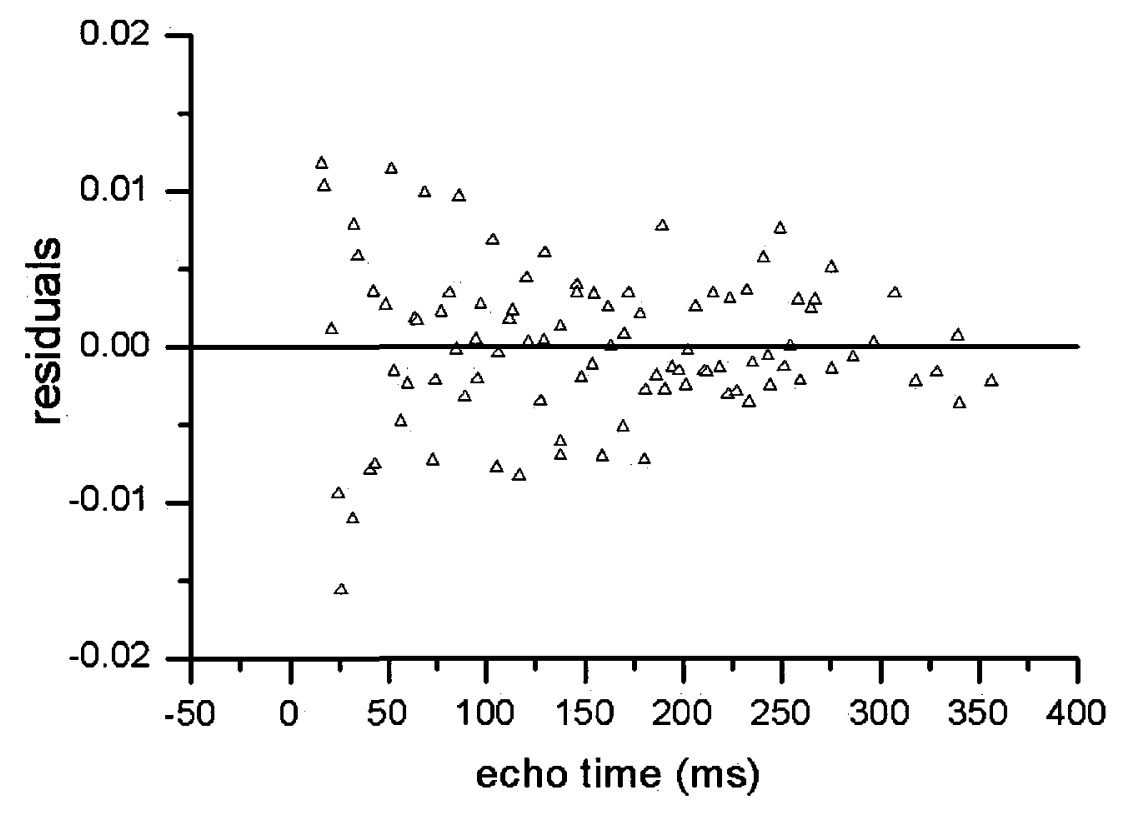

Figure 5.3: A typical plot of the residuals from a tri-exponential fit of a $T_{2}$ decay.

Prediction residuals, an alternative measure of fitting accuracy, are plotted in Fig. 5.3. The standard deviation of these residuals decreases for the later echo times; however, at shorter echo times, there remains a systematic oscillation, usually smaller than $0.02 \%$ of the $T E=0$ intercept of the decay curve. This small systematic variation, which can be understood in terms of slightly imperfect $180^{\circ}$ pulses or possibly spurious stimulated echoes was similar 
for all volumes in the image and in turn had a negligible effect on the resulting $T_{2}$ values obtained from the curves.

\subsection{Diffusion Measurements}

The DTI measurements showed that the maximum fractional anisotropy $(F A)$ values for the measured slice were in the splenium of the corpus callosum (SCC); the values in the genu of the corpus callosum (GCC) were almost as high. However, in both cases they were lower in the 51 year old subject than they were in the 36 year old subject, as expected. The mean $F A$ for the SCC and GCC of the 51 year old subject, averaged over all the measurements reported here were $0.794 \pm 0.005$ and $0.777 \pm 0.021$, respectively. Similarly, for the 36 year

old subject the mean $F A$ values averaged over all measurements were $0.887 \pm 0.002$ and 0.865 \pm 0.002 for the SCC and GCC, respectively.

Fig. 5.4 shows typical RB corrected and uncorrected experimental diffusion decay curves, together with their corresponding biexponential fits, for the selected ROI in the SCC of the 51 year old subject with the diffusion sensitizing gradient in each of the left-right (LR) and inferior-superior (IS) directions. The decay curves, plotted here as the signal to noise ratio (SNR) versus the $b$-value, display non-exponential behaviour and a marked directional dependence. The decay curves for the 36 year old subject (not shown) did not differ in any significant way from the curves shown in Fig. 5.4. The deviation from exponentiality for the LR decay is substantial whereas it is more subtle for the IS decay. Rician bias contributes significantly to this deviation from exponentiality, especially for the LR curve. 


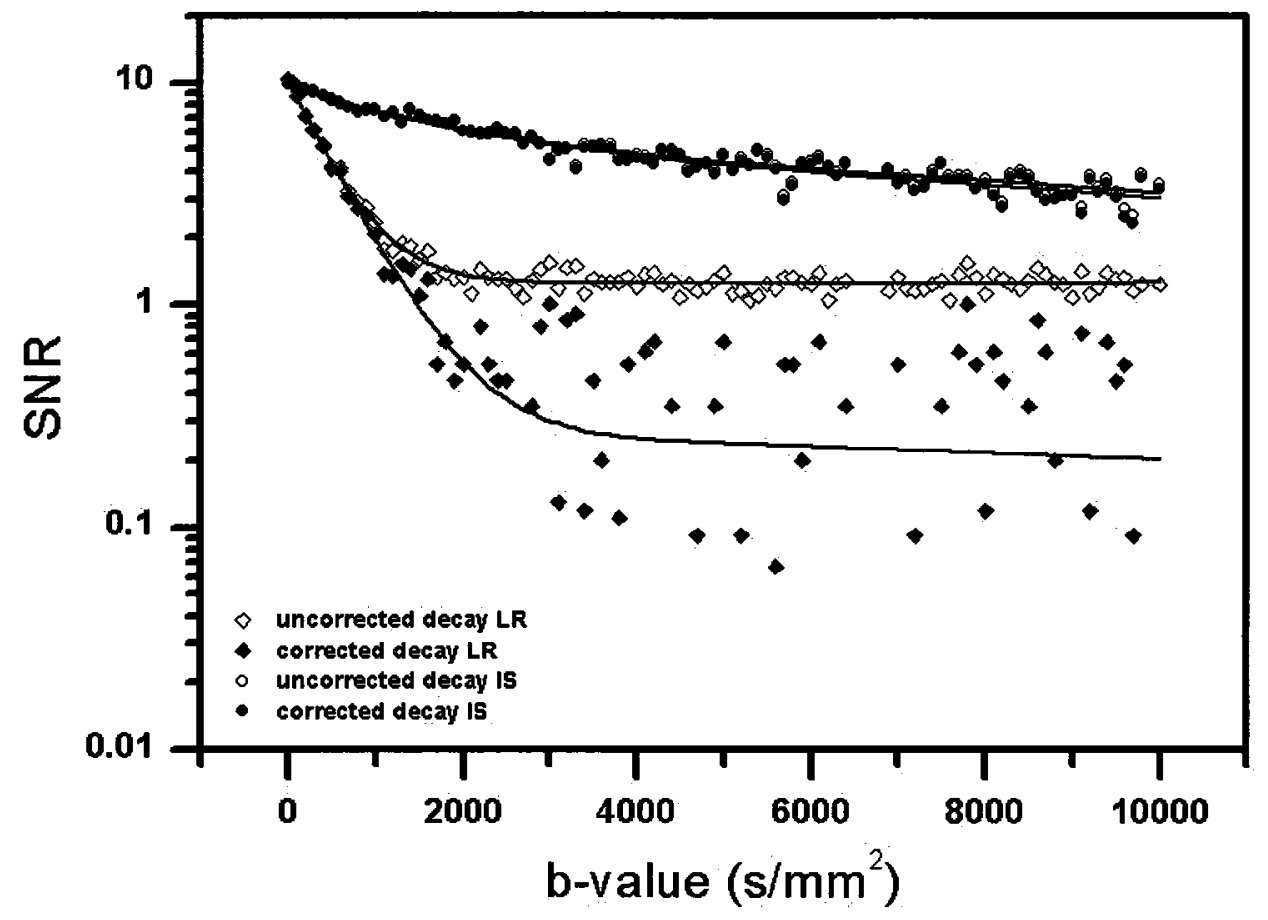

Figure 5.4: Semi-logarithmic plot of SNR vs. $b$-values measured in the LR (diamonds) and IS (circles) directions for a 9-pixel ROI located within the SCC of the 51 year old subject before and after RB correction (open and solid symbols, respectively). The solid curves represent the optimal biexponential fits.

The measured diffusion decays were all fit to a biexponential decay function. In all cases $R^{2}$ values very close to one were obtained, giving the confidence that these decays are well characterized by a biexponential function.

For the LR data from the SCC of the 51 year old subject the initial SNR (i.e. for $b=0$ $\mathrm{s} / \mathrm{mm}^{2}$ ) for all curves was in the range 9.5 to 10.5 for all decay curves. Before RB correction, the decay was clearly non-exponential, while the biexponentiality of the corrected decay was much less pronounced. The noise floor occurs at about $12 \%$ of the initial signal (SNR $=1.2$ ) and a $b$-value of about $2000 \mathrm{~s} / \mathrm{mm}^{2}$; however, $\mathrm{RB}$ can be seen up to almost $20 \%$ of the initial 
signal which corresponds to $b$-values of about $1500 \mathrm{~s} / \mathrm{mm}^{2}$ in these experiments. RB correction reduced the noise floor by a factor of 6 to $\mathrm{SNR}=0.2$ allowing the diffusion decay to be analyzed without the effects of RB out to $b=2000 \mathrm{~s} / \mathrm{mm}^{2}$. However, the RB correction increased the scatter in the data points.

For the IS data the signal decays much more slowly and the effect of Rician bias is not as significant. However, there is evidence of the subtle RB effects for $b$-values $>8000 \mathrm{~s} / \mathrm{mm}^{2}$ which corresponding to about $35 \%$ of the initial signal and a SNR of about 3.5 .

\subsubsection{Diffusion Parallel to the Axons in the SCC and the GCC}

When the diffusion sensitization gradients are applied parallel to the axonal direction (i.e. LR in the subject frame of reference for the experiments reported here) the signal decays quickly with increasing $b$-values. Tables 5.4 (a) and (b) give the fit parameters for the 9 trials before and after RB correction for the 51 year old subject. The fast and slow components, after $\mathrm{RB}$ correction, are characterized, on average, by $D_{\text {fast }}=(1.81 \pm 0.08) \times 10^{-3} \mathrm{~mm}^{2} / \mathrm{s}$ and $D_{\text {slow }}=$ $(0.09 \pm 0.03) \times 10^{-3} \mathrm{~mm}^{2} / \mathrm{s}$, respectively and have mean signal fractions of $0.95 \pm 0.01$ and 0.04 \pm 0.01 , respectively. The goodness of fit $\left(R^{2}\right)$ was very good in all cases. All of the fit parameters are significantly different for the RB corrected curves compared to the uncorrected curves. Similar results are reported for the 36 year old volunteer in Tables 5.5 (a) and (b).

The experiments were done in groups of 3 experiments in each of 3 sessions to test for intra-session and inter-session variability. In Tables 5.4 to 5.6, the three sessions consisted of trials 1 to 3,4 to 6 and 7 to 9 , respectively. Between trials within a given session the subject was removed from the scanner. The results for all trials are very consistent. Based on a visual inspection of the diffusion decays and the fit parameters given in Tables 5.4 to 5.6, it was 


\begin{tabular}{|ccccccc|}
\hline trials & $\boldsymbol{f}_{\text {fast }}$ & $\begin{array}{c}\boldsymbol{D}_{\text {fast }} \\
\times 10^{-3} \mathrm{~mm}^{2} / \mathrm{s}\end{array}$ & $\boldsymbol{f}_{\text {slow }}$ & $\begin{array}{c}\boldsymbol{D}_{\text {slow }} \\
\times 10^{-3} \mathrm{~mm}^{2} / \mathrm{s}\end{array}$ & FA & $\boldsymbol{R}^{2}$ \\
\hline 1 & 0.85 & 2.00 & 0.12 & 0.002 & 0.83 & 0.99 \\
2 & 0.85 & 2.30 & 0.13 & 0.001 & 0.76 & 0.99 \\
3 & 0.88 & 2.13 & 0.12 & 0.002 & 0.89 & 0.99 \\
4 & 0.86 & 2.25 & 0.14 & 0.001 & 0.90 & 0.99 \\
5 & 0.87 & 2.23 & 0.14 & 0.005 & 0.78 & 0.99 \\
6 & 0.88 & 2.00 & 0.13 & 0.003 & 0.76 & 1.00 \\
7 & 0.88 & 2.19 & 0.12 & 0.002 & 0.78 & 0.99 \\
8 & 0.87 & 2.06 & 0.13 & 0.001 & 0.81 & 0.99 \\
9 & 0.90 & 2.11 & 0.12 & 0.007 & 0.78 & 0.99 \\
mean & $\mathbf{0 . 8 7}$ & $\mathbf{2 . 1 4}$ & $\mathbf{0 . 1 3}$ & $\mathbf{0 . 0 0 3}$ & $\mathbf{0 . 8 1}$ & \\
SD & $\mathbf{0 . 0 2}$ & $\mathbf{0 . 1 1}$ & $\mathbf{0 . 0 1}$ & $\mathbf{0 . 0 0 2}$ & $\mathbf{0 . 0 5}$ & \\
\hline
\end{tabular}

a).

\begin{tabular}{|ccccccc|}
\hline trials & $\boldsymbol{f}_{\text {fast }}$ & $\begin{array}{c}\boldsymbol{D}_{\text {fast }} \\
\times 10^{-3} \mathrm{~mm}^{2} / \mathrm{s}\end{array}$ & $\boldsymbol{f}_{\text {slow }}$ & $\begin{array}{c}\boldsymbol{D}_{\text {slow }} \\
\times 10^{-3} \mathrm{~mm}^{2} / \mathrm{s}\end{array}$ & FA & $\boldsymbol{R}^{2}$ \\
\hline 1 & 0.95 & 1.87 & 0.04 & 0.11 & 0.83 & 0.97 \\
2 & 0.93 & 1.88 & 0.04 & 0.10 & 0.76 & 0.95 \\
3 & 0.96 & 1.75 & 0.03 & 0.11 & 0.89 & 0.97 \\
4 & 0.94 & 1.89 & 0.04 & 0.07 & 0.90 & 0.96 \\
5 & 0.95 & 1.86 & 0.05 & 0.12 & 0.78 & 0.96 \\
6 & 0.94 & 1.75 & 0.07 & 0.11 & 0.76 & 0.97 \\
7 & 0.94 & 1.88 & 0.05 & 0.06 & 0.78 & 0.97 \\
8 & 0.94 & 1.71 & 0.04 & 0.05 & 0.81 & 0.96 \\
9 & 0.98 & 1.72 & 0.02 & 0.06 & 0.78 & 0.97 \\
mean & $\mathbf{0 . 9 5}$ & $\mathbf{1 . 8 1}$ & $\mathbf{0 . 0 4}$ & $\mathbf{0 . 0 9}$ & $\mathbf{0 . 8 1}$ & \\
SD & $\mathbf{0 . 0 1}$ & $\mathbf{0 . 0 8}$ & $\mathbf{0 . 0 1}$ & $\mathbf{0 . 0 3}$ & $\mathbf{0 . 0 5}$ & \\
\hline
\end{tabular}

b).

Table 5.4: Experimental results for the SCC of the 51 year old subject with diffusion sensitization in the LR direction for uncorrected (a) and corrected (b) normalized signal decay curves including the mean and standard deviation (SD) for the 9 trials. The reported fit parameters are from the best fit to a biexponential function. The goodness of fit $\left(R^{2}\right)$ and $F A$ values for each trial are also provided. 


\begin{tabular}{|ccccccc|}
\hline trials & $\boldsymbol{f}_{\text {fast }}$ & $\begin{array}{c}\boldsymbol{D}_{\text {fast }} \\
\mathrm{x} 10^{-3} \mathrm{~mm}^{2} / \mathrm{s}\end{array}$ & $\boldsymbol{f}_{\text {slow }}$ & $\begin{array}{c}\boldsymbol{D}_{\text {slow }} \\
\times 10^{-3} \mathrm{~mm}^{2} / \mathrm{s}\end{array}$ & FA & $\boldsymbol{R}^{2}$ \\
\hline 1 & 0.91 & 2.16 & 0.11 & 0.005 & 0.83 & 0.99 \\
2 & 0.89 & 2.19 & 0.11 & 0.002 & 0.86 & 0.99 \\
3 & 0.88 & 2.23 & 0.12 & 0.002 & 0.91 & 0.99 \\
4 & 0.88 & 2.26 & 0.11 & 0.002 & 0.93 & 0.99 \\
5 & 0.89 & 2.18 & 0.13 & 0.003 & 0.95 & 0.99 \\
6 & 0.91 & 2.26 & 0.12 & 0.006 & 0.88 & 0.99 \\
7 & 0.88 & 2.22 & 0.12 & 0.009 & 0.92 & 1.00 \\
8 & 0.88 & 2.24 & 0.12 & 0.007 & 0.88 & 1.00 \\
9 & 0.88 & 2.29 & 0.12 & 0.005 & 0.89 & 0.99 \\
mean & $\mathbf{0 . 8 9}$ & $\mathbf{2 . 2 3}$ & $\mathbf{0 . 1 2}$ & $\mathbf{0 . 0 0 5}$ & $\mathbf{0 . 8 9}$ & \\
SD & $\mathbf{0 . 0 1}$ & $\mathbf{0 . 0 4}$ & $\mathbf{0 . 0 1}$ & $\mathbf{0 . 0 0 3}$ & $\mathbf{0 . 0 4}$ & \\
\hline
\end{tabular}

a).

\begin{tabular}{|ccccccc|}
\hline trials & $\boldsymbol{f}_{\text {fast }}$ & $\begin{array}{c}\boldsymbol{D}_{\text {fast }} \\
\times 10^{-3} \mathrm{~mm}^{2} / \mathrm{s}\end{array}$ & $\boldsymbol{f}_{\text {slow }}$ & $\begin{array}{c}\boldsymbol{D}_{\text {slow }} \\
\times 10^{-3} \mathrm{~mm}^{2} / \mathrm{s}\end{array}$ & FA & $\boldsymbol{R}^{2}$ \\
\hline 1 & 0.95 & 1.91 & 0.05 & 0.07 & 0.83 & 0.97 \\
2 & 0.96 & 1.81 & 0.05 & 0.09 & 0.86 & 0.98 \\
3 & 0.94 & 1.98 & 0.04 & 0.07 & 0.91 & 0.97 \\
4 & 0.94 & 1.97 & 0.05 & 0.09 & 0.93 & 0.98 \\
5 & 0.96 & 1.86 & 0.02 & 0.07 & 0.95 & 0.96 \\
6 & 0.97 & 2.01 & 0.03 & 0.07 & 0.88 & 0.97 \\
7 & 0.91 & 1.98 & 0.08 & 0.10 & 0.92 & 0.98 \\
8 & 0.93 & 2.00 & 0.06 & 0.11 & 0.88 & 0.97 \\
9 & 0.95 & 1.89 & 0.04 & 0.08 & 0.89 & 0.97 \\
mean & $\mathbf{0 . 9 5}$ & $\mathbf{1 . 9 3}$ & $\mathbf{0 . 0 5}$ & $\mathbf{0 . 0 8}$ & $\mathbf{0 . 8 9}$ & \\
SD & $\mathbf{0 . 0 2}$ & $\mathbf{0 . 0 7}$ & $\mathbf{0 . 0 2}$ & $\mathbf{0 . 0 2}$ & $\mathbf{0 . 0 4}$ & \\
\hline
\end{tabular}

b).

Table 5.5: Experimental results for the SCC of the 36 year old subject with diffusion sensitization in the LR direction for uncorrected (a) and corrected (b) normalized signal decay curves including the mean and standard deviation (SD) for the 9 trials. The reported fit parameters are from the best fit to a biexponential function. The goodness of fit $\left(R^{2}\right)$ and $F A$ values for each trial are also provided. 


\begin{tabular}{|ccccccc|}
\hline trials & $\boldsymbol{f}_{\text {fast }}$ & $\begin{array}{c}\boldsymbol{D}_{\text {fast }} \\
\times 10^{-3} \mathrm{~mm}^{2} / \mathrm{s}\end{array}$ & $\boldsymbol{f}_{\text {slow }}$ & $\begin{array}{c}\boldsymbol{D}_{\text {slow }} \\
\times 10^{-3} \mathrm{~mm}^{2} / \mathrm{s}\end{array}$ & FA & $\boldsymbol{R}^{2}$ \\
\hline 1 & 0.95 & 1.63 & 0.03 & 0.06 & 0.78 & 0.94 \\
2 & 0.94 & 1.81 & 0.04 & 0.11 & 0.78 & 0.95 \\
3 & 0.90 & 1.87 & 0.10 & 0.08 & 0.80 & 0.96 \\
4 & 0.95 & 1.80 & 0.05 & 0.07 & 0.79 & 0.96 \\
5 & 0.90 & 1.76 & 0.08 & 0.12 & 0.81 & 0.96 \\
6 & 0.95 & 1.81 & 0.03 & 0.11 & 0.83 & 0.97 \\
7 & 0.97 & 1.66 & 0.02 & 0.06 & 0.78 & 0.95 \\
8 & 0.96 & 1.81 & 0.04 & 0.08 & 0.76 & 0.96 \\
9 & 0.99 & 1.71 & 0.02 & 0.11 & 0.84 & 0.96 \\
mean & $\mathbf{0 . 9 5}$ & $\mathbf{1 . 7 6}$ & $\mathbf{0 . 0 5}$ & $\mathbf{0 . 0 9}$ & $\mathbf{0 . 8 0}$ & \\
SD & $\mathbf{0 . 0 3}$ & $\mathbf{0 . 0 8}$ & $\mathbf{0 . 0 3}$ & $\mathbf{0 . 0 2}$ & $\mathbf{0 . 0 3}$ & \\
\hline
\end{tabular}

a).

\begin{tabular}{|ccccccc|}
\hline trials & $\boldsymbol{f}_{\text {fast }}$ & $\begin{array}{c}\boldsymbol{D}_{\text {fast }} \\
\times 10^{-3} \mathrm{~mm}^{2} / \mathrm{s}\end{array}$ & $\boldsymbol{f}_{\text {slow }}$ & $\begin{array}{c}\boldsymbol{D}_{\text {slow }} \\
\times 10^{-3} \mathrm{~mm}^{2} / \mathrm{s}\end{array}$ & FA & $\boldsymbol{R}^{\mathbf{2}}$ \\
\hline 1 & 0.97 & 1.79 & 0.04 & 0.11 & 0.87 & 0.96 \\
2 & 0.98 & 1.83 & 0.04 & 0.04 & 0.88 & 0.97 \\
3 & 0.98 & 1.77 & 0.03 & 0.02 & 0.89 & 0.97 \\
4 & 0.96 & 1.83 & 0.07 & 0.12 & 0.86 & 0.97 \\
5 & 0.97 & 1.84 & 0.04 & 0.07 & 0.89 & 0.97 \\
6 & 0.97 & 1.87 & 0.02 & 0.08 & 0.87 & 0.96 \\
7 & 0.94 & 1.81 & 0.04 & 0.10 & 0.86 & 0.98 \\
8 & 0.94 & 1.80 & 0.04 & 0.10 & 0.88 & 0.98 \\
9 & 0.97 & 1.79 & 0.02 & 0.07 & 0.89 & 0.96 \\
mean & $\mathbf{0 . 9 6}$ & $\mathbf{1 . 8 1}$ & $\mathbf{0 . 0 4}$ & $\mathbf{0 . 0 8}$ & $\mathbf{0 . 8 8}$ & \\
SD & $\mathbf{0 . 0 2}$ & $\mathbf{0 . 0 3}$ & $\mathbf{0 . 0 1}$ & $\mathbf{0 . 0 3}$ & $\mathbf{0 . 0 1}$ & \\
\hline
\end{tabular}

b).

Table 5.6: Experimental results for the GCC with diffusion sensitization in the LR direction. Only the corrected normalized signal decay curves including the mean and standard deviation (stdev) for the 9 trials are reported here for (a) the 51 year old subject and (b) the 36 year old subject. The reported fit parameters are from the best fit to a biexponential function. The goodness of fit $\left(R^{2}\right)$ and $F A$ values for each trial are also provided. 
concluded that there was no significant inter-session variability and that the intra-session variance was consistent with the variance for the complete set of trials.

Table 5.6 shows that the experiments measured in the GCC also produced signal attenuation curves that can be satisfactorily fitted with a biexponential decay model. These results for the GCC are not statistically different between the volunteers and once again, the brain water appeared to be dominated mainly by the fast diffusing pool $\left(f_{\text {fast }} \approx 95-96 \%\right)$ with a mean $D_{\text {fast }} \approx 1.8 \times 10^{-3} \mathrm{~mm}^{2} / \mathrm{s}$.

\subsubsection{Diffusion Perpendicular to the Axons in the SCC and the GCC}

When the diffusion sensitization gradients are applied perpendicular to the axonal direction (i.e. inferior-superior, (IS) in the subject frame of reference for the experiments reported here) the signal decays slowly and there is still a significant amount of signal available at high $b$-values. RB correction had an almost negligible effect in this case. For example, Table 5.7 gives the fit parameters for the SCC averaged over the 8 trials before and after RB correction for the 51 year old subject. The fast and slow components are characterized, on average, by $D_{\text {fast }}=(0.65 \pm 0.11) \times 10^{-3} \mathrm{~mm}^{2} / \mathrm{s}$ and $D_{\text {slow }}=(0.06 \pm 0.01) \times 10^{-3}$ $\mathrm{mm}^{2} / \mathrm{s}$, respectively and have mean signal fractions of $0.42 \pm 0.05$ and $0.56 \pm 0.06$,

respectively. The goodness of fit $\left(R^{2}\right)$ was very good in all cases. Similar results for the SCC are also reported for the 36 year old subject (see Table 5.8).

These experiments were done in a similar manner to the parallel case. For both volunteers a group of 3 experiments was performed in each of 3 sessions (with the exception of the final session where only 2 trials were done) to test for intra-session and inter-session variability. In Tables 5.7 to 5.9 , the three sessions consisted of trials 1 to 3,4 to 6 and 7 to 8 , 


\begin{tabular}{|ccccccc|}
\hline trials & $\boldsymbol{f}_{\text {fast }}$ & $\begin{array}{c}\boldsymbol{D}_{\text {fast }} \\
\times 10^{-3} \mathrm{~mm}^{2} / \mathrm{s}\end{array}$ & $\boldsymbol{f}_{\text {slow }}$ & $\begin{array}{c}\boldsymbol{D}_{\text {slow }} \\
\times 10^{-3} \mathrm{~mm}^{2} / \mathrm{s}\end{array}$ & FA & $\boldsymbol{R}^{2}$ \\
\hline 1 & 0.51 & 0.48 & 0.43 & 0.03 & 0.79 & 0.96 \\
2 & 0.46 & 0.62 & 0.53 & 0.05 & 0.82 & 0.97 \\
3 & 0.37 & 0.59 & 0.57 & 0.05 & 0.80 & 0.94 \\
4 & 0.43 & 0.64 & 0.56 & 0.06 & 0.79 & 0.96 \\
5 & 0.38 & 0.87 & 0.61 & 0.07 & 0.78 & 0.95 \\
6 & 0.38 & 0.64 & 0.60 & 0.06 & 0.78 & 0.94 \\
7 & 0.41 & 0.68 & 0.58 & 0.05 & 0.78 & 0.93 \\
8 & 0.46 & 0.61 & 0.52 & 0.04 & 0.84 & 0.95 \\
mean & $\mathbf{0 . 4 3}$ & $\mathbf{0 . 6 4}$ & $\mathbf{0 . 5 5}$ & $\mathbf{0 . 0 5}$ & $\mathbf{0 . 8 0}$ & \\
SD & $\mathbf{0 . 0 5}$ & $\mathbf{0 . 1 2}$ & $\mathbf{0 . 0 6}$ & $\mathbf{0 . 0 1}$ & $\mathbf{0 . 0 2}$ & \\
\hline
\end{tabular}

a).

\begin{tabular}{|ccccccc|}
\hline trials & $\boldsymbol{f}_{\text {fast }}$ & $\begin{array}{c}\boldsymbol{D}_{\text {fast }} \\
\times 10^{-3} \mathrm{~mm}^{2} / \mathrm{s}\end{array}$ & $\boldsymbol{f}_{\text {slow }}$ & $\begin{array}{c}\boldsymbol{D}_{\text {slow }} \\
\times 10^{-3} \mathrm{~mm}^{2} / \mathrm{s}\end{array}$ & FA & $\boldsymbol{R}^{2}$ \\
\hline 1 & 0.51 & 0.48 & 0.42 & 0.03 & 0.79 & 0.96 \\
2 & 0.46 & 0.62 & 0.53 & 0.05 & 0.82 & 0.97 \\
3 & 0.37 & 0.59 & 0.57 & 0.05 & 0.80 & 0.94 \\
4 & 0.43 & 0.64 & 0.56 & 0.06 & 0.79 & 0.96 \\
5 & 0.38 & 0.87 & 0.61 & 0.07 & 0.78 & 0.95 \\
6 & 0.38 & 0.64 & 0.60 & 0.06 & 0.78 & 0.94 \\
7 & 0.41 & 0.68 & 0.58 & 0.06 & 0.78 & 0.93 \\
8 & 0.41 & 0.71 & 0.57 & 0.06 & 0.84 & 0.96 \\
mean & $\mathbf{0 . 4 2}$ & $\mathbf{0 . 6 5}$ & $\mathbf{0 . 5 6}$ & $\mathbf{0 . 0 6}$ & $\mathbf{0 . 8 0}$ & \\
SD & $\mathbf{0 . 0 5}$ & $\mathbf{0 . 1 1}$ & $\mathbf{0 . 0 6}$ & $\mathbf{0 . 0 1}$ & $\mathbf{0 . 0 2}$ & \\
\hline
\end{tabular}

b).

Table 5.7: Experimental results for the SCC of the 51 year old subject with diffusion sensitization in the IS direction for uncorrected (a) and corrected (b) normalized signal decay curves including the mean and standard deviation (SD) over the 8 trials. The reported fit parameters are from the best fit to a biexponential function. The goodness of fit $\left(R^{2}\right)$ and $F A$ values for each trial are also provided. 


\begin{tabular}{|ccccccc|}
\hline trials & $\boldsymbol{f}_{\text {fast }}$ & $\begin{array}{c}\boldsymbol{D}_{\text {fast }} \\
\times 10^{-3} \mathrm{~mm}^{2} / \mathrm{s}\end{array}$ & $\boldsymbol{f}_{\text {slow }}$ & $\begin{array}{c}\boldsymbol{D}_{\text {slow }} \\
\times 10^{-3} \mathrm{~mm}^{2} / \mathrm{s}\end{array}$ & FA & $\boldsymbol{R}^{2}$ \\
\hline 1 & 0.36 & 0.66 & 0.66 & 0.03 & 0.85 & 0.93 \\
2 & 0.46 & 0.78 & 0.50 & 0.06 & 0.83 & 0.98 \\
3 & 0.26 & 0.75 & 0.72 & 0.06 & 0.87 & 0.96 \\
4 & 0.23 & 0.65 & 0.75 & 0.05 & 0.85 & 0.96 \\
5 & 0.47 & 0.44 & 0.54 & 0.04 & 0.83 & 0.98 \\
6 & 0.35 & 0.67 & 0.77 & 0.04 & 0.85 & 0.93 \\
7 & 0.32 & 0.63 & 0.67 & 0.05 & 0.89 & 0.94 \\
8 & 0.34 & 0.52 & 0.68 & 0.05 & 0.90 & 0.97 \\
mean & $\mathbf{0 . 3 5}$ & $\mathbf{0 . 6 4}$ & $\mathbf{0 . 6 6}$ & $\mathbf{0 . 0 5}$ & $\mathbf{0 . 8 6}$ & \\
SD & $\mathbf{0 . 0 8}$ & $\mathbf{0 . 1 1}$ & $\mathbf{0 . 1 0}$ & $\mathbf{0 . 0 1}$ & $\mathbf{0 . 0 3}$ & \\
\hline
\end{tabular}

a).

\begin{tabular}{|ccccccc|}
\hline trials & $\boldsymbol{f}_{\text {fast }}$ & $\begin{array}{c}\boldsymbol{D}_{\text {fast }} \\
\times 10^{-3} \mathrm{~mm}^{2} / \mathrm{s}\end{array}$ & $\boldsymbol{f}_{\text {slow }}$ & $\begin{array}{c}\boldsymbol{D}_{\text {slow }} \\
\times 10^{-3} \mathrm{~mm}^{2} / \mathrm{s}\end{array}$ & $\mathbf{F A}$ & $\boldsymbol{R}^{2}$ \\
\hline 1 & 0.36 & 0.66 & 0.65 & 0.03 & 0.85 & 0.98 \\
2 & 0.46 & 0.78 & 0.50 & 0.06 & 0.83 & 0.98 \\
3 & 0.26 & 0.75 & 0.72 & 0.06 & 0.87 & 0.96 \\
4 & 0.23 & 0.66 & 0.75 & 0.06 & 0.85 & 0.96 \\
5 & 0.47 & 0.45 & 0.54 & 0.05 & 0.83 & 0.98 \\
6 & 0.35 & 0.67 & 0.77 & 0.04 & 0.85 & 0.93 \\
7 & 0.32 & 0.64 & 0.67 & 0.06 & 0.89 & 0.98 \\
8 & 0.30 & 0.53 & 0.68 & 0.05 & 0.90 & 0.97 \\
mean & $\mathbf{0 . 3 4}$ & $\mathbf{0 . 6 4}$ & $\mathbf{0 . 6 6}$ & $\mathbf{0 . 0 5}$ & $\mathbf{0 . 8 6}$ & \\
SD & $\mathbf{0 . 0 9}$ & $\mathbf{0 . 1 1}$ & $\mathbf{0 . 1 0}$ & $\mathbf{0 . 0 1}$ & $\mathbf{0 . 0 3}$ & \\
\hline
\end{tabular}

b).

Table 5.8: Experimental results for the SCC of the 36 year old subject with diffusion sensitization in the IS direction for uncorrected (a) and corrected (b) normalized signal decay curves including the mean and standard deviation (SD) over the 8 trials. The reported fit parameters are from the best fit to a biexponential function. The goodness of fit $\left(R^{2}\right)$ and $F A$ values for each trial are also provided. 


\begin{tabular}{|ccccccc|}
\hline trials & $\boldsymbol{f}_{\text {fast }}$ & $\begin{array}{c}\boldsymbol{D}_{\text {fast }} \\
\times 10^{-3} \mathrm{~mm}^{2} / \mathrm{s}\end{array}$ & $\boldsymbol{f}_{\text {slow }}$ & $\begin{array}{c}\boldsymbol{D}_{\text {slow }} \\
\times 10^{-3} \mathrm{~mm}^{2} / \mathrm{s}\end{array}$ & FA & $\boldsymbol{R}^{2}$ \\
\hline 1 & 0.55 & 0.70 & 0.43 & 0.09 & 0.82 & 0.98 \\
2 & 0.60 & 0.54 & 0.39 & 0.04 & 0.77 & 0.98 \\
3 & 0.59 & 0.73 & 0.45 & 0.05 & 0.77 & 0.96 \\
4 & 0.57 & 0.61 & 0.45 & 0.07 & 0.80 & 0.98 \\
5 & 0.63 & 0.57 & 0.41 & 0.04 & 0.83 & 0.97 \\
6 & 0.46 & 0.66 & 0.52 & 0.06 & 0.74 & 0.98 \\
7 & 0.60 & 0.76 & 0.38 & 0.07 & 0.77 & 0.98 \\
8 & 0.55 & 0.75 & 0.46 & 0.04 & 0.77 & 0.98 \\
mean & $\mathbf{0 . 5 7}$ & $\mathbf{0 . 6 7}$ & $\mathbf{0 . 4 4}$ & $\mathbf{0 . 0 6}$ & $\mathbf{0 . 7 8}$ & \\
SD & $\mathbf{0 . 0 5}$ & $\mathbf{0 . 0 8}$ & $\mathbf{0 . 0 4}$ & $\mathbf{0 . 0 2}$ & $\mathbf{0 . 0 3}$ & \\
\hline
\end{tabular}

a).

\begin{tabular}{|ccccccc|}
\hline trials & $\boldsymbol{f}_{\text {fast }}$ & $\begin{array}{c}\boldsymbol{D}_{\text {fast }} \\
\times 10^{-3} \mathrm{~mm}^{2} / \mathrm{s}\end{array}$ & $\boldsymbol{f}_{\text {slow }}$ & $\begin{array}{c}\boldsymbol{D}_{\text {slow }} \\
\times 10^{-3} \mathrm{~mm}^{2} / \mathrm{s}\end{array}$ & $\mathbf{F A}$ & $\boldsymbol{R}^{\mathbf{2}}$ \\
\hline 1 & 0.45 & 0.62 & 0.53 & 0.06 & 0.82 & 0.98 \\
2 & 0.59 & 0.75 & 0.60 & 0.07 & 0.82 & 0.96 \\
3 & 0.47 & 0.62 & 0.50 & 0.05 & 0.86 & 0.97 \\
4 & 0.47 & 0.72 & 0.53 & 0.06 & 0.84 & 0.97 \\
5 & 0.41 & 0.66 & 0.60 & 0.07 & 0.87 & 0.96 \\
6 & 0.49 & 0.67 & 0.61 & 0.07 & 0.83 & 0.96 \\
7 & 0.52 & 0.57 & 0.46 & 0.06 & 0.86 & 0.98 \\
8 & 0.47 & 0.65 & 0.52 & 0.07 & 0.82 & 0.97 \\
mean & $\mathbf{0 . 4 8}$ & $\mathbf{0 . 6 6}$ & $\mathbf{0 . 5 4}$ & $\mathbf{0 . 0 6}$ & $\mathbf{0 . 8 4}$ & \\
SD & $\mathbf{0 . 0 5}$ & $\mathbf{0 . 0 6}$ & $\mathbf{0 . 0 5}$ & $\mathbf{0 . 0 1}$ & $\mathbf{0 . 0 2}$ & \\
\hline
\end{tabular}

b).

Table 5.9: Experimental results for the GCC with diffusion sensitization in the IS direction. Only the corrected normalized signal decay curves including the mean and standard deviation (stdev) for the 8 trials are reported here for (a) the 51 year old subject and (b) the 36 year old subject. The reported fit parameters are from the best fit to a biexponential function. The goodness of fit $\left(R^{2}\right)$ and $F A$ values for each trial are also provided.

respectively. Between trials within a given session the subjects were removed from the scanner. The results for all trials are very consistent. Based on a visual inspection of the diffusion decays and the fit parameters given in Tables 5.7 to 5.9, it was concluded that there was no significant inter-session variability and that the intra-session variance was consistent 
with the variance for the complete set of trials. From Table 5.9 it can be seen that the experiments measured in the GCC also produce decay curves that can be satisfactorily fitted to a biexponential decay model. These results are not statistically different between the volunteers. The water in the GCC appears to be about equally divided between the fast and slow diffusion pools.

\subsubsection{Diffusion Orientation Dependence in the SCC and the GCC}

Samples of normalized diffusion decays for the SCC are shown in Fig. 5.5 as a function of $\alpha$, the angle between the diffusion sensitization gradient direction and the $x$-axis of the scanner (i.e. LR in the patient reference frame), which is considered to be parallel to the axonal direction in the SCC and the GCC. The diffusion decay was found to be very sensitive to $\alpha$; the measured diffusion decays change considerably for $\alpha$ between $30-90^{\circ}$ and more subtly for $\alpha<30^{\circ}$. The decay curves were normalized to the signal intensity acquired with $b=$ 0 to improve visualization of subtle differences especially for $\alpha<30^{\circ}$ where the decay curves are more similar. The decays measured for the GCC (not shown) were very similar; their qualitative behaviour does not differ in any significant way from the curves shown in Fig. 5.5.

From Table 5.10 it can be seen that $D_{\text {slow }}$ for the SCC remains approximately constant, to within experimental error, for smaller angles but decreases as $\alpha$ increases from about $45^{\circ}$ to $90^{\circ}$. This trend is subtle and is barely beyond experimental error. The values of $D_{\text {slow }}$ obtained for the GCC (see Table 5.11) appear to have the same trend but, if such a trend does exist for the GCC, it could not be resolved beyond experimental error. The $D_{\text {slow }}$ values averaged over all orientations for the SCC are $0.10 \times 10^{-3} \mathrm{~mm}^{2} / \mathrm{s}$ and $0.09 \times 10^{-3} \mathrm{~mm}^{2} / \mathrm{s}$ for the 51 and 36 year old subjects, respectively, and $0.09 \times 10^{-3} \mathrm{~mm}^{2} / \mathrm{s}$ for the GCC for both subjects. The very small 
values of $D_{\text {slow }}$ observed for both the SCC and the GCC are more than an order of magnitude smaller than the diffusion coefficient for pure water but fall within the range of previously reported values for the translational mobility of water in hydration layers [145].

$D_{\text {fast }}$ decreases with increasing $\alpha$ for both the SCC and the GCC and for both subjects. This behavior, which was observed for both subjects, is consistent with the well known diffusion anisotropy of white matter that has been previously reported. This trend can be clearly seen in Tables 5.10 and 5.11 and in Fig. 5.6.

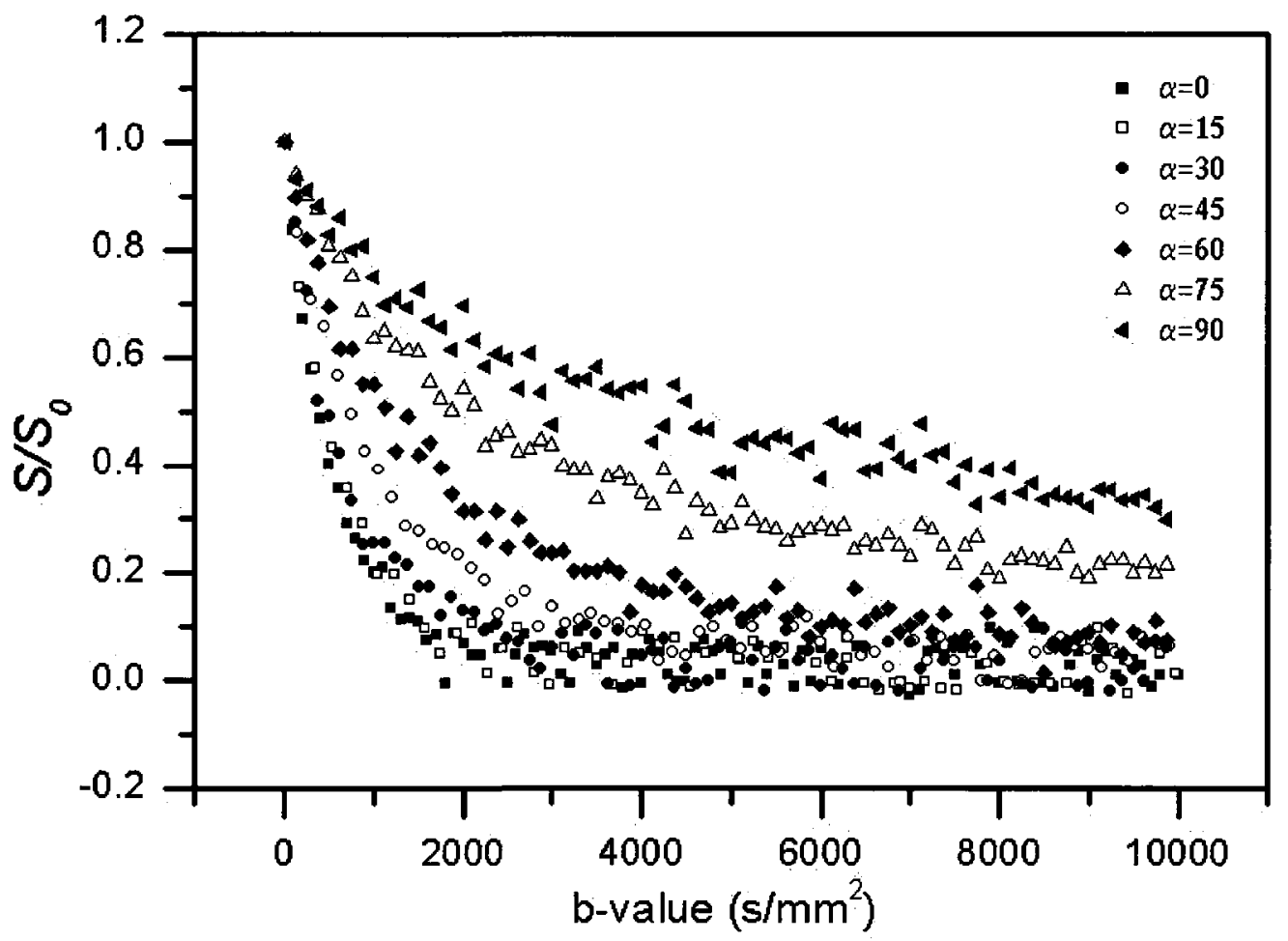

Figure 5.5: Normalized, RB corrected diffusion attenuation curves from the SCC of the 51 year old subject for $\alpha=0^{\circ}$ to $90^{\circ}$. 


\begin{tabular}{|cccccc|}
\hline $\begin{array}{c}\text { angle } \\
\boldsymbol{\alpha}\end{array}$ & $\boldsymbol{f}_{\text {fast }}$ & $\begin{array}{c}\boldsymbol{D}_{\text {fast }} \\
\times 10^{-3} \mathrm{~mm}^{2} / \mathrm{s}\end{array}$ & $f_{\text {slow }}$ & $\begin{array}{c}D_{\text {slow }} \\
\times 10^{-3} \mathrm{~mm}^{2} / \mathrm{s}\end{array}$ & FA \\
\hline 0 & $0.95 \pm 0.01$ & $1.81 \pm 0.08$ & $0.04 \pm 0.01$ & $0.09 \pm 0.03$ & $0.80 \pm 0.04$ \\
15 & $0.95 \pm 0.02$ & $1.69 \pm 0.06$ & $0.05 \pm 0.02$ & $0.12 \pm 0.03$ & $0.79 \pm 0.03$ \\
30 & $0.92 \pm 0.03$ & $1.50 \pm 0.06$ & $0.07 \pm 0.02$ & $0.13 \pm 0.05$ & $0.79 \pm 0.01$ \\
45 & $0.87 \pm 0.02$ & $1.10 \pm 0.08$ & $0.12 \pm 0.03$ & $0.12 \pm 0.04$ & $0.80 \pm 0.02$ \\
60 & $0.74 \pm 0.04$ & $0.82 \pm 0.05$ & $0.25 \pm 0.03$ & $0.11 \pm 0.01$ & $0.79 \pm 0.03$ \\
75 & $0.52 \pm 0.08$ & $0.70 \pm 0.06$ & $0.46 \pm 0.07$ & $0.08 \pm 0.01$ & $0.79 \pm 0.02$ \\
90 & $0.42 \pm 0.05$ & $0.65 \pm 0.11$ & $0.56 \pm 0.06$ & $0.06 \pm 0.01$ & $0.80 \pm 0.02$ \\
\hline
\end{tabular}

a).

\begin{tabular}{|cccccc|}
\hline $\begin{array}{c}\text { angle } \\
\boldsymbol{\alpha}\end{array}$ & $\boldsymbol{f}_{\text {fast }}$ & $\begin{array}{c}\boldsymbol{D}_{\text {fast }} \\
\mathrm{x} 10^{-3} \mathrm{~mm}^{2} / \mathrm{s}\end{array}$ & $\boldsymbol{f}_{\text {slow }}$ & $\begin{array}{c}\boldsymbol{D}_{\text {slow }} \\
\times 10^{-3} \mathrm{~mm}^{2} / \mathrm{s}\end{array}$ & $\mathbf{F A}$ \\
\hline 0 & $0.95 \pm 0.02$ & $1.93 \pm 0.07$ & $0.05 \pm 0.02$ & $0.08 \pm 0.02$ & $0.89 \pm 0.04$ \\
30 & $0.95 \pm 0.03$ & $1.50 \pm 0.11$ & $0.05 \pm 0.03$ & $0.08 \pm 0.06$ & $0.89 \pm 0.02$ \\
45 & $0.89 \pm 0.01$ & $1.19 \pm 0.10$ & $0.10 \pm 0.02$ & $0.14 \pm 0.04$ & $0.92 \pm 0.03$ \\
60 & $0.71 \pm 0.09$ & $0.83 \pm 0.08$ & $0.29 \pm 0.07$ & $0.11 \pm 0.03$ & $0.86 \pm 0.04$ \\
75 & $0.47 \pm 0.12$ & $0.68 \pm 0.05$ & $0.51 \pm 0.12$ & $0.08 \pm 0.02$ & $0.90 \pm 0.02$ \\
90 & $0.34 \pm 0.09$ & $0.64 \pm 0.11$ & $0.66 \pm 0.10$ & $0.05 \pm 0.01$ & $0.86 \pm 0.02$ \\
\hline
\end{tabular}

b).

Table 5.10: Fast and slow diffusion coefficients for the SCC of (a) the 51 and (b) the 36 year old subjects, respectively, reported as mean \pm SD along with their corresponding signal fractions, also reported as mean $\pm \mathrm{SD}$, derived from the biexponential fit for $\alpha=0-90^{\circ}$. The $F A$ values averaged over 6 independent trials for each $\alpha$ are also reported as average \pm SD. 


\begin{tabular}{|c|c|c|c|c|c|}
\hline $\begin{array}{c}\text { angle } \\
\boldsymbol{\alpha}\end{array}$ & $f_{\text {fast }}$ & $\begin{array}{c}\boldsymbol{D}_{\text {fast }} \\
\times 10^{-3} \mathrm{~mm}^{2} / \mathrm{s}\end{array}$ & $f_{\text {slow }}$ & $\begin{array}{c}\boldsymbol{D}_{\text {slow }} \\
\times 10^{-3} \mathrm{~mm}^{2} / \mathrm{s}\end{array}$ & FA \\
\hline 0 & $0.95 \pm 0.03$ & $1.76 \pm 0.08$ & $0.05 \pm 0.03$ & $0.09 \pm 0.02$ & $0.80 \pm 0.03$ \\
\hline 15 & $0.94 \pm 0.03$ & $1.69 \pm 0.06$ & $0.05 \pm 0.02$ & $0.07 \pm 0.02$ & $0.79 \pm 0.02$ \\
\hline 30 & $0.92 \pm 0.03$ & $1.51 \pm 0.12$ & $0.06 \pm 0.02$ & $0.10 \pm 0.04$ & $0.75 \pm 0.02$ \\
\hline 45 & $0.92 \pm 0.03$ & $1.14 \pm 0.06$ & $0.08 \pm 0.03$ & $0.15 \pm 0.07$ & $0.80 \pm 0.02$ \\
\hline 60 & $0.83 \pm 0.08$ & $0.86 \pm 0.08$ & $0.19 \pm 0.07$ & $0.09 \pm 0.04$ & $0.76 \pm 0.03$ \\
\hline 75 & $0.70 \pm 0.05$ & $0.75 \pm 0.12$ & $0.31 \pm 0.05$ & $0.08 \pm 0.02$ & $0.78 \pm 0.02$ \\
\hline 90 & $0.57 \pm 0.05$ & $0.67 \pm 0.08$ & $0.44 \pm 0.04$ & $0.06 \pm 0.02$ & $0.76 \pm 0.03$ \\
\hline
\end{tabular}

a).

\begin{tabular}{|c|c|c|c|c|c|}
\hline $\begin{array}{c}\text { angle } \\
\alpha\end{array}$ & $\overline{f_{\text {fast }}}$ & $\begin{array}{c}D_{\text {fast }} \\
\times 10^{-3} \mathrm{~mm}^{2} / \mathrm{s}\end{array}$ & $\overline{f_{\text {slow }}}$ & $\begin{array}{c}\boldsymbol{D}_{\text {slow }} \\
\times 10^{-3} \mathrm{~mm}^{2} / \mathrm{s}\end{array}$ & $\overline{\mathbf{F A}}$ \\
\hline 0 & $0.96 \pm 0.02$ & $1.81 \pm 0.03$ & $0.04 \pm 0.01$ & $0.08 \pm 0.03$ & $0.88 \pm 0.01$ \\
\hline 30 & $0.93 \pm 0.02$ & $1.57 \pm 0.11$ & $0.05 \pm 0.01$ & $0.10 \pm 0.03$ & $0.86 \pm 0.03$ \\
\hline 45 & $0.93 \pm 0.02$ & $1.18 \pm 0.06$ & $0.04 \pm 0.01$ & $0.07 \pm 0.03$ & $0.89 \pm 0.03$ \\
\hline 60 & $0.80 \pm 0.06$ & $0.91 \pm 0.12$ & $0.20 \pm 0.06$ & $0.11 \pm 0.04$ & $0.84 \pm 0.03$ \\
\hline 75 & $0.67 \pm 0.11$ & $0.70 \pm 0.08$ & $0.31 \pm 0.09$ & $0.09 \pm 0.03$ & $0.88 \pm 0.02$ \\
\hline 90 & $0.48 \pm 0.05$ & $0.66 \pm 0.06$ & $0.54 \pm 0.05$ & $0.06 \pm 0.01$ & $0.84 \pm 0.02$ \\
\hline
\end{tabular}

b).

Table 5.11: Fast and slow diffusion coefficients for the GCC of (a) the 51 and (b) the 36 year old subjects, respectively, reported as mean $\pm S D$, along with their corresponding signal fractions, also reported as mean $\pm \mathrm{SD}$, derived from the biexponential fit for $\alpha=0-90^{\circ}$. The $F A$ values averaged over 6 independent trials for each $\alpha$ are also reported as average \pm SD.

There is a shift in balance between the fast and slow component fractions, when the direction of the gradient is systematically varied. There is a general tendency of increasing 
slow water fraction, $f_{\text {slow }}$ at the expense of the fast water fraction $f_{\text {fast }}$, as $\alpha$ increases (see Table 5.10 and Figs. 5.6 and 5.7). Thus, there is not only anisotropy in $D_{\text {fast }}$ (and possibly $D_{\text {slow }}$ ) but also in $f_{\text {fast }}$ and $f_{\text {slow. }}$ This was observed for both the SCC and the GCC in both volunteers; however, the anisotropy of $f_{\text {fast }}$ and $f_{\text {slow }}$ was more pronounced for the SCC than it was for the GCC.

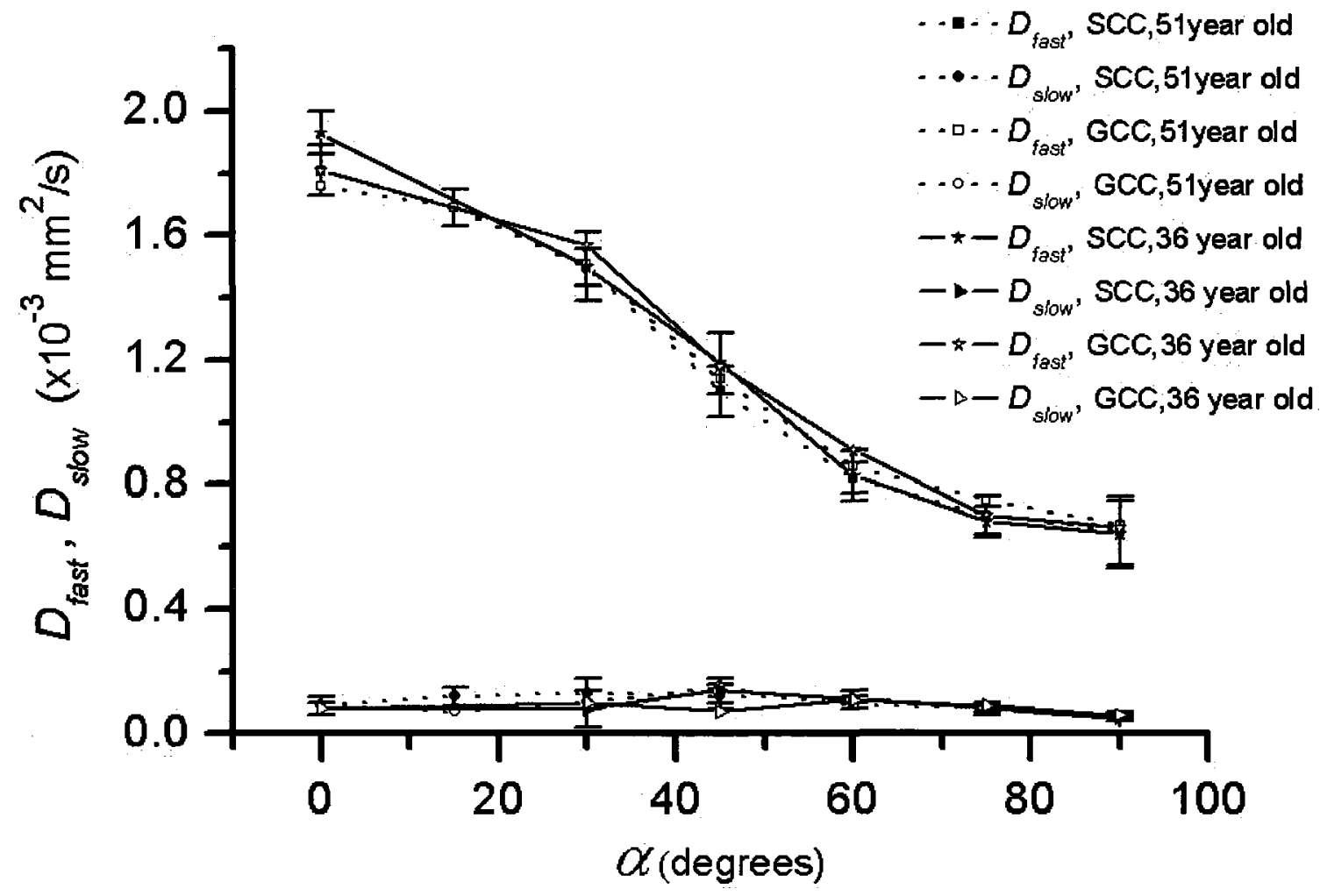

Figure 5.6: Variations of $D_{\text {fast }}$ and $D_{\text {slow }}$ plotted as a function of $\alpha$. The error bars refer to the standard deviation of the fitted parameter obtained from the Marquardt-Levenberg fit. 

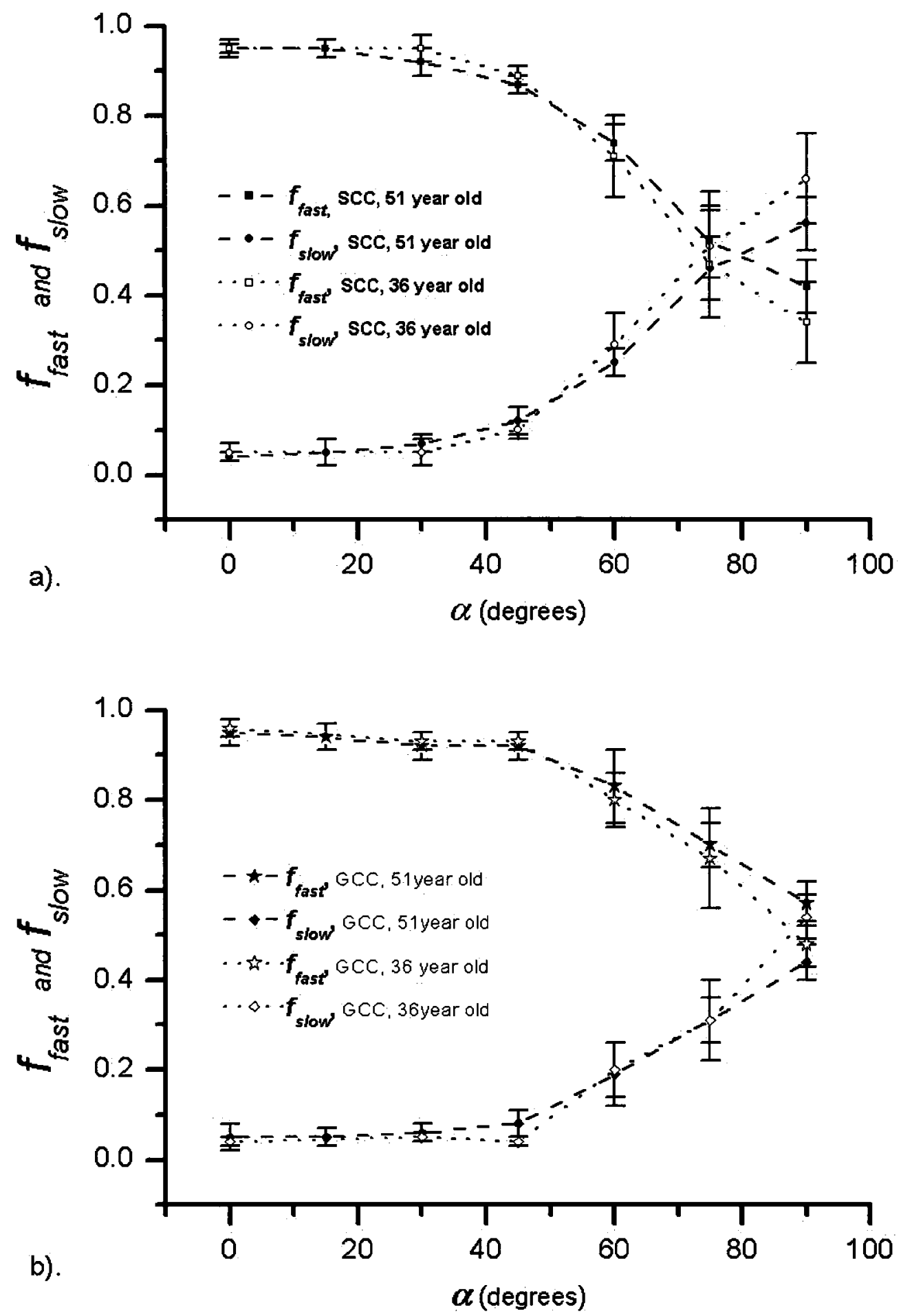

Figure 5.7: Changes in $f_{\text {fast }}$ and $f_{\text {slow }}$ plotted as function of $\alpha$ for the SCC (a) and the GCC (b). The error bars refer to the standard deviation of the fitted parameter obtained from the Marquardt-Levenberg fit. 
In spite of the anatomical similarities between the genu and the splenium of the corpus callosum, there is a significant difference in their relative slow and fast signal fractions when diffusion is measured across the axonal membrane (i.e. $\alpha=90^{\circ}$ ) while the values for both $D_{\text {fast }}$ and $D_{\text {slow }}$ stay remarkably close (see Fig. 5.6), as expected. No statistically significant differences were observed between the volunteers for $D_{\text {fast }}$ and $D_{\text {slow }}$ in either the SCC or the GCC. 


\section{Chapter 6}

\section{Discussions}

In this thesis carefully measured sets of diffusion decays for the SCC and GCC of two healthy subjects in vivo are presented along with supporting $T_{2}$ relaxation time measurements. The diffusion decays were measured as function of the angle between the diffusion direction and the long axis of the axon. Strong anisotropy effects were observed for both the diffusion coefficients and the associated signal fractions.

The diffusion decays were measured for $b$-values up to $10,000 \mathrm{~s} / \mathrm{mm}^{2}$. At these large $b$-values the decays are affected by Rician Bias (RB). Without properly correcting the decays for RB effects, quantitative analysis and interpretation of the decays is meaningless. Until recently, no reliable method existed for the removal of this bias. However, an RB correction method which is analytically exact, as long as accurate values for the mean and standard 
deviation of the signal probability density function are available, has recently been published [159]. A method for implementing this method when only approximate values of the true mean and standard deviation are known has also recently been proposed [163]. An improved version of this implementation strategy has been developed and presented in Chapter 3 of this thesis.

DWI provides one of the most attractive techniques for investigating molecular transport in biological tissues since it permits molecules to be labeled and tracked without perturbing their motion. To assess the divergent theories proposed in the literature for explaining tissue water dynamics, it is important to understand precisely what is being measured with DWI. Traditionally, diffusion decay components have been interpreted in terms of restricted diffusion models with two well defined compartments: the intracellular space and the extracellular space. However, these models do not account for all of the observed results; in particular, the observed signal fractions are inconsistent with this model. Many studies have experimentally established that the overall low diffusivity of water in cells cannot be fully explained by compartmentalization or restriction effects caused by cell membranes or by scattering or obstruction and tortuosity effects from cellular macromolecules, microtubules and neurofilaments [20-22].

In this thesis the effect on diffusion of the interactions of water molecules with each other and with their surroundings, particularly with polar sidegroups of macromolecules in the cell and incorporated into the cell's membranes, are also considered. These interactions lead to the formation of hydration layers around macromolecules and along membrane surfaces and cause the water in the cell to behave more like a gel that a liquid. Tissue water is often said to be more "structured" or "ordered" than bulk water $[122,133]$. These considerations have allowed developing novel explanations for: 1) why the measured fast diffusion component is 
smaller than the diffusion coefficient of free water, 2) why it is anisotropic, 3) the observed signal fraction values and 4) the anisotropy of the signal fractions.

Evaluation of water diffusion in the corpus callosum, CC, unambiguously revealed fast and slow diffusion components; therefore the assignment of these components to two distinct water pools is reasonable. If this interpretation is correct, then one of these pools consists of water which is close enough to macromolecular or membrane surfaces that its diffusional motion is affected by interactions between the polar water molecules and the charged molecular sidegroups on the surface of the macromolecule or membrane (i.e. the hydration layers). The other pool consists of water that is far enough from this surface that it preserves its bulk state properties.

The long range translational apparent diffusion coefficient has been, for a long time, regarded as a probe of tissue morphology or cell ultrastructure but has not been considered to provide much useful information about water mobility at the local level. So far, the huge amount of in vivo brain DWI data available in the literature has mostly been interpreted with the view that water molecules in tissue diffuse as if they were in pure water until they meet a barrier, such as the cell membrane, which simply reflects the particle back in a very classical sense. This is unfortunate since the properties of hydration water could have the most important influence on the observed diffusion behavior in cells in vivo. While it cannot be denied that the internal structure of the cell and the membranes surrounding the cell pose obstructions to diffusion, the electrostatic interactions of the polar water molecules with their surroundings must also have a significant effect on water diffusion in the cell. Even in the absence of direct obstruction effects, the diffusion of water molecules in cells will be perturbed by hydration water associated with these obstacles. 
Water structure is a multifaceted property that has not been fully characterized experimentally even for bulk water and the dynamic structure of hydration water is understood even less. Trantham et al [166] used quasielastic neutron scattering to study water diffusion in cells. The method gives information on the water diffusion rate over short distances (a few angstroms) corresponding to a time interval of $10^{-12} \mathrm{~s}$. Thus, this method does not allow enough time for the average water molecule to encounter barriers to diffusion except those due to the water molecules themselves [167]. They measured a very small diffusion coefficient of $\sim 10^{-5} \mathrm{~mm}^{2} / \mathrm{s}$, about two orders of magnitude smaller than the diffusion coefficient for free, liquid water. This is quite inconsistent with the notion that the water molecules diffuse as if they were in their free liquid state until they encounter an obstacle. It is much more consistent with the notion of a structured gel-like cytoplasm. This method has not been widely applied to cells and tissues since most cannot tolerate the necessary experimental conditions.

The following discussion attempts to correctly explain the observed non-Gaussian diffusion behaviour in white matter tissue by considering the polarity of the water molecules. In particular, the slower decay observed at high $b$-values, which is often interpreted as an increased level of geometrical restriction, could instead be associated with a population of water molecules that has an inherently slower diffusion rate and is not able to move far enough to be dephased in spite of the large diffusion weighting. This would be consistent with water in hydration shells. Furthermore, the faster diffusion coefficient being smaller than the diffusion coefficient for pure water also consistent with the fact that the hydrogen bonded gellike network of water molecules in the cell inhibits diffusion. The anisotropy of diffusion in white matter can also be explained using this approach since diffusion in hydration layers has previously been reported to be anisotropic [145]. Moreover, it supports the proposal of non- 
bulklike diffusion of cellular water to properly account for the reduction in water mobility as compared to bulk water.

\section{$6.1 \mathrm{~T}_{2}$ Measurements}

The measurement of $T_{2}$ relaxation times with MR has the potential to be a powerful tool to evaluate the state of water in tissue because the relaxation times are influenced by the chemical and physical environment surrounding each individual water molecule. Unfortunately, there seems to be little agreement in the literature on how many $T_{2}$ components there are in white matter, what their values are and to which pool of water they belong (see Section 6.1.1). In particular, reliable $T_{2}$ relaxation studies specifically for the SCC and GCC in humans in vivo are not available in the literature. Disappointingly, without an appropriate model quantitative relaxation time information is of little use clinically.

With the above motivations the present study was initiated to: 1) determine quantitatively, the multiple relaxation times in human corpus callosum in vivo, 2) determine the water signal fraction associated with each relaxation time, 3) assign the measured components to water in specific tissue environments and 4) to propose a physical model to explain these findings. The first two points are discussed in Section 6.1.2 of and the last two are discussed in Sections 6.1.3 and 6.1.4.

To achieve these goals, high quality $T_{2}$ data sets were required. The $T_{2}$ relaxation decay curves reported in this study were very carefully measured using many $T E$ values from $8.1 \mathrm{~ms}$ to $320 \mathrm{~ms}$ (although the first reliable data point was at $16.2 \mathrm{~ms}-$ see Section 5.1). An additional experiment that followed the decay to $510 \mathrm{~ms}$, where the signal strength had fallen to about $2 \%$ of its initial value, was also performed and no significant differences were found 
in the fit parameters. The signal-to-noise ratio (SNR) for $T E=16.2 \mathrm{~ms}$ was about $350: 1$. The $T_{2}$ decays were highly reproducible, both when the measurements were repeated in the same session and when they were from different sessions. Consequently, we believe that the decay curves presented in this study and the analysis of those curves are very reliable.

Results for the two subjects studied were in good agreement to within experimental error. Age dependent effects have been reported in the literature for certain areas of the brain where increased iron concentration in the tissue and in the walls of blood vessels is associated with normal aging [168]. Histochemical, histopathological and MR imaging studies have determined that maximum iron concentration in normal adults is found in the globus pallidus, putamen, caudate nucleus, thalamus, cortical grey and cortical white matter. This progressive increase of iron content with age may be related to a combination of factors; mainly decreased oxidative phosphorylation, declining oligodendroglial function and an accelerated hydroxyl free radical formation with lipid membrane peroxidation [168]. This effect does not become apparent in $T_{2}$-weighted MR images until the sixth decade of life. Although the older subject in this study is in his early fifties, no age dependent effects were expected or observed in the SCC or the GCC.

In spite of the high quality of the $T_{2}$ decay curves reported here, an unambiguously determination of whether the $T_{2}$ decay is best characterized as a bi-exponential or a triexponential decay was not possible, based on the goodness of fit. A similar difficulty is encountered when surveying the literature since some groups claim that there are only two $T_{2}$ components while others claim that there are three components. 


\subsection{1 $\mathrm{T}_{2}$ Decay Curve Analysis}

The ability of MR scanners to perform $T_{2}$ relaxation measurements precisely and accurately has improved over time. In spite of this, most studies in the literature have only been able to confirm that $T_{2}$ decay curves obtained in neural tissues, both in vitro and in vivo, are well-suited to multiexponential analyses. The components are generally interpreted in terms of different water compartments with some contributions from macromolecules, particularly for the very short $T_{2}$ components [169-172] but the nature of these proposed compartments remains uncertain.

However, there is a general consensus regarding the $T_{2}$ relaxation components measured for brain tissues and several studies have dealt with their assignment $[98,100,171]$. The $T_{2}$ decay curve decomposition for peripheral nerve yields three primary components with $T_{2}$ values of approximately 20,80 and $250 \mathrm{~ms}$. These components are generally assigned to water protons in the myelin, intracellular water and extracellular water, respectively. For normal human brain the component with the largest signal fraction and $T_{2}$ values of approximately $70-90 \mathrm{~ms}$, has been assigned to intra/extracellular water. The short $T_{2}$ component, with values between 10 and $50 \mathrm{~ms}$, has a much smaller signal fraction and is considered to be due to water in the myelin sheath. The longest $T_{2}$ component is generally associated with cerebrospinal fluid (CSF). It is also believed that SNR limitations on current scanners are likely responsible for the difficulty in separating the intra/extracellular water component.

While there is enough evidence to support the assignment of the shortest $T_{2}$ component to myelin water [173-175], the origin of the two longer components still remains unclear. Comparison between components and compartments has been used in some studies to suggest 
that the intermediate component results from intra-axonal water, and the longest component is from inter-axonal water [176]. However, a similar study [51] has shown that this assignment should be reversed. More intriguingly, some $T_{2}$ decays measured in white matter show only two components, and do not exhibit the longest component $[165,169,176]$. Other studies report a prolonged $T_{2}$ component in heavily myelinated white matter structures [177] and in MS lesions [178]. In these studies, the largest component, $70<T_{2}<200 \mathrm{~ms}$, has been assigned to intra/extracellular water while the smaller component $10<T_{2}<50 \mathrm{~ms}$ was assigned to water trapped between the bilayers of the myelin sheath.

Although the microscopic nature of peripheral nerve is not the same as that of white matter, it could be anticipated that all white matter and grey matter structures should have similar $T_{2}$ behaviour. However, this was proved to be untrue. $T_{2}$ relaxation measurements for individual brain structures reveal markedly different water and myelin water contents (studies have regularly reported fractions of myelin water up to $20 \%$ ) and different white and grey matter structures decay at dissimilar rates [178]. Moreover, it had been noted that several brain pathologies give rise to unique decay curves $[175,179,180]$.

\subsubsection{Interpretation of $\mathrm{T}_{2}$ Components}

$T_{2}$ relaxation is caused primarily by transitions among the Zeeman energy levels of the spin system. These transitions are induced by fluctuating magnetic fields experienced by the spins due to dipole-dipole interactions. When these fields fluctuate at the Larmor frequency, transitions among the Zeeman energy levels are induced. The dipolar interactions that lead to these fluctuating fields can be grouped into three types: 1) intramolecular interactions, 2) intermolecular interactions between hydrogen nuclei on different water molecules and 3) the 
interaction of water molecules with magnetic dipoles on molecules other than water (e.g. proteins). The relevant background theory of dipole-dipole induced $T_{2}$ relaxation is reviewed in section 2.5. In pure bulk water the intramolecular contribution dominates since the intermolecular contributions are averaged out on the MRI time scale by the rapid rotational motion of the water molecules. However, even the intramolecular interaction is weakened by the rapid rotational motion of the water molecules.

In aqueous protein solutions, water molecules interact with the polar side groups on the protein molecule. This has two important effects on the $T_{2}$ relaxation. First of all, many of these side groups generate local magnetic fields which have a spectral component fluctuating at the Larmor frequency that can induce $T_{2}$ relaxation directly. Secondly, the electrostatic interactions of the water molecules with these ligands restrict the motion of the water molecules such that motional averaging is significantly reduced. Consequently, intramolecular and intermolecular dipolar interactions for the water molecules increase substantially. In addition, these oriented polar water molecules cause a certain degree of rotational order in the next layer of water molecules, and these act to orient the next layer, and so on. This has an important effect in orienting the water molecules for at least three layers of water molecules, with the effect diminishing as the distance from the ligand increases. In dilute aqueous protein solutions, the $T_{2}$ decay is bi-exponential with the longer component corresponding to bulk water and shorter one corresponding to hydration water. In more concentrated protein solutions, the decay is dominated by hydration water and the $T_{2}$ is very short. 


\subsubsection{The Short $T_{2}$ Component}

The short $T_{2}$ component in the SCC has mean values of $16 \pm 1$ and $18 \pm 3 \mathrm{~ms}$ with corresponding signal fractions of $10 \pm 1 \%$ and $11 \pm 2 \%$ for the older and younger subject, respectively. Similarly, in the GCC the mean $T_{2}$ value was found to be $13 \pm 2 \mathrm{~ms}$ for both subjects and the signal fractions were $15 \pm 4 \%$ and $13 \pm 5 \%$ for the 51 and 36 year old subjects, respectively.

In order for this relaxation time to be so short, the water associated with this component must either be close to many strong relaxation inducing sites, or "structured" such that intramolecular dipolar interactions are not completely averaged out, or both. The water must experience a fluctuating magnetic field with a strong intensity at the Larmor frequency in order to have such efficient relaxation. The source of these fluctuating fields is likely a combination of macromolecular side group motion and increased intermolecular and intramolecular dipolar interactions due to restricted motion of the water molecules. Macromolecular side groups (i.e. $\mathrm{CH}_{3}, \mathrm{NH}_{3}, \ldots$ ) generate fluctuating magnetic fields as they undergo their pseudo-random motion associated with rotational and vibrational degrees of freedom. These fluctuating magnetic fields induce $T_{2}$ relaxation in water molecules that are nearby, with the effect decreasing with distance from the side group. In normal bulk water, at body temperature, intermolecular dipolar interactions are essentially zero and intramolecular dipolar interactions are reduced on the MR time scale due to averaging associated with the very fast rotational motion of the water molecules causing the $T_{2}$ for bulk water to be quite long. When the rotational motion of water molecules is restricted, as is the case in hydration layers associated with macromolecules such as, for example, the many proteins which protrude 
from the interior and exterior surfaces of cell membranes. These dipolar interactions are stronger and the $T_{2}$ for hydration water, as a result, is much shorter than for bulk water.

The most probable location of a large portion of the water that contributes to this short component is, as suggested by previous studies, $[51,176,181]$ within the myelin sheaths, regardless of the number of layers, since each layer is nearly identical. Myelin has a much higher lipid content [182] (78-81\% of the dry weight) than white matter (49-66\%) or even grey matter (30-40\%), which makes it more disposed to interact with all tissue water in its vicinity. The dominant contribution to this $T_{2}$ component would undoubtedly come from water that interacts directly with the abundant myelin polar groups, the first hydration layer adjacent to the multiple lipid bilayers radially wound around the long axis of axons. Furthermore, we should be perfectly safe in referring to this tightly bound water as being "trapped" inside the myelin sheath. The apparent effect is that these water molecules are "buried" in a closed space and cannot escape due to geometrical confinement. Since the radial distance from one membrane to the next in the myelin sheath is very small, all of the water inside the sheath will be hydration water. Given the condition of this rigid bound state, not far below the rigidbinding limit of unity [183], their fluctuating fields and dipolar interactions are not totally averaged by their motion. As a result, their relaxation time would be drastically reduced.

The tightly bound water state is assumed to be quite stable due to a decreased mobility of the water molecules found in this particular state. To leave this bound state, the water molecules have to pass through a high-energy state where the water-protein hydrogen bonds are broken before new water-water hydrogen bonds can be formed. The implication of a long residence time in this bound state is consistent with the classic view of limited permeability of 
myelin, likely indicating that the myelin water does not exchange with intra-axonal or interaxonal water to a significant degree on the time scale of a $T_{2}$ experiment.

The short- $T_{2}$ component in white matter has only recently been associated with myelin water. Previously, it had been consistently ascribed to water which is closely associated with and strongly bound to proteins and/or phospholipids in almost all qualitative studies of brain tissue, whether in vivo or in vitro, human or non-human. While this statement is no doubt correct, it was not identified until recently that the majority of the hydration water associated with this short $T_{2}$ component in white matter is probably confined within the myelin sheath. A similar short- $T_{2}$ component has also been observed in grey matter [184], but the fraction of the water pool associated with it showed more variation and was always significantly smaller than the one corresponding to white matter structures.

\subsubsection{The Long $T_{2}$ Component}

The long $T_{2}$ component in the SCC has mean values of $196 \pm 33 \mathrm{~ms}$, (signal fraction of $17 \pm 6 \%$ ) for the 51 year old subject and $173 \pm 24 \mathrm{~ms}$, (signal fraction of $23 \pm 6 \%$ ) for the 36 year old subject. Similarly, in the GCC the mean $T_{2}$ values were $364 \pm 109 \mathrm{~ms}$, with signal fraction of $15 \pm 7 \%$ and $245 \pm 84 \mathrm{~ms}$ with signal fraction of $14 \pm 8 \%$, for the 51 and 36 year old subjects, respectively.

The water fraction that is associated with the long $T_{2}$ component is not affected directly by the protein-lipid polar groups and yet the apparent transverse relaxation time for this component is still very much less than that for normal bulk water. This water is likely similar in its physical properties to the non-hydration water in a dilute aqueous protein solution (although it will, of course, also have access to membranes, connective tissue, etc.). In such a 
system $T_{2}$ will almost certainly be lowered, relative to bulk water, via exchange with weakly bound hydration water. In fact, one could argue that in living cells water is never far from an interface suggesting that most of the cellular water is influenced by the macromolecular interface given the time frame of a $T_{2}$ experiment.

The corpus callosum is a crowded molecular structure with high axonal density. The SCC, in particular, has thick fibers [118] and is heavily myelinated to ensure high speed neuronal transmissions. In general, myelin constitutes more than $50 \%$ of the dry mass of white matter tissue. Therefore, due to the abundance of axonal membranes and high myelin content in the SCC, most of the tissue water can be expected to be bound to some extent. This would also suggest that the signal fraction associated with the long $T_{2}$ component should be relatively small, consistent with the observations reported here.

The existence of this $T_{2}$ component has been disputed by some researchers who maintain that it is an artifact of the analysis procedure [185] while others support its existence. In muscle tissue, for example, Belton et al [186] found a long $T_{2}$ component with a fraction of approximately $15 \%$ and assigned it to water in the extracellular volume. Hazlewood et al [187] distinguished a $10 \%$ fraction of water protons associated with $T_{2}=196 \mathrm{~ms}$ in muscle tissue at $24^{\circ} \mathrm{C}$. They suggested that this component should be associated with water in the extracellular space which did not exchange rapidly with the intracellular water.

\subsubsection{The Intermediate $T_{2}$ Component}

The intermediate $T_{2}$ component in the SCC has mean values of $76 \pm 4 \mathrm{~ms}$, (signal fraction of $74 \pm 5 \%$ ) for the 51 year old subject and $71 \pm 5 \mathrm{~ms}$, (signal fraction of $67 \pm 4 \%$ ) for the 36 year old subject. Similarly, in the GCC the mean $T_{2}$ values were $85 \pm 6 \mathrm{~ms}$, with signal 
fraction of $69 \pm 5 \%$ and $72 \pm 6 \mathrm{~ms}$ with signal fraction of $72 \pm 5 \%$, for the 51 and 36 year old subjects, respectively. The signal fraction associated with the intermediate $T_{2}$ component is approximately $70 \%$ of the total signal for both ROIs and both subjects. This large component corresponds to water that is not strongly affected by the protein-lipid charged groups but whose apparent $T_{2}$ value is still very much less than that for normal bulk water.

It is somewhat surprising that there should be such a clear cut experimental distinction between the short and intermediate relaxation time components. Since they are physically not separated by a barrier of any sort, one might have expected a gradual falling off of the influence of the polar interfaces. In this case the separation into two distinct water pools is determined by the residence time of the water molecules at the macromolecular surface and the exchange between different hydration layers. Evidence that intracellular water exists in a multilayer form in muscle tissue has been provided by Ling and Negendank [188] from vapor pressure studies. Additional evidence supporting a collective water structure model comes from dielectric measurements [189]. Quantum mechanical calculations have suggested that the hydrogen bonding of water molecules is a cooperative process.

\subsubsection{Hydration Layer and Disordered Zone Model}

The first hydration layer lies immediately adjacent to macromolecular surfaces and consists of water molecules hydrogen bonded to polar side groups on the macromolecular surface. The effect of these polar groups will be felt over several layers of water molecules through the polarizing effect of one hydration layer on the next. These hydration layers, between the first hydration layer and the a distance far enough from the macromolecule that its effect is not longer felt, directly or indirectly, will be referred to here as the disordered zone. 
The water in the disordered zone is characterized by decreased rates of rotational motion as compared to tissue bulk water. The nature and extent of the ordering of water molecules immediately adjacent to the interface will determine the extent of the disordered zone. It has been estimated that as much as $50 \%$ of the total cell water may be associated with the cytoskeleton if the hydration layer is of the order of $3 \mathrm{~nm}$ thick (about 10 molecules thick) [122].

It must be emphasized that the structural water entities (i.e. hydration layers) are not stable, permanent structures. In fact, they experience frequent disruptions and alterations. But they present similar geometrically identifiable features wherever they occur in the cell. An essential point then is the temporal stability of such identifiable characteristics. The implication is that the hydration water structure may have a lifetime which is notably longer than the corresponding lifetime of the structured entity in weakly bound bulk water. The effective relaxation rates will be related to the behavior of water in each of these structural states, i.e. tightly or weakly bound water and bulk-like water since the intensity of the magnetic fluctuations which induce the relaxation depends on the details of the restricted rotations and translations at each location of the water molecules with respect to the polar macromolecular surfaces.

Normally, at low levels of water content, all water in the tissue exists in a tightly bound form. This is obvious in view of the available sites for hydrogen bonding and strong iondipole interaction. At a medium level of water content, loosely bound water exists in addition to tightly bound water, which already occupies the available sites for strong interaction. Cells that contain less than 75 to $80 \%$ water by mass can be expected to have no pure-bulk water [122]. Free water begins to appear when the water content is raised to still higher levels. 


\begin{tabular}{lccc}
\hline Component & Whole human brain $(\%)$ & Grey matter $(\%)$ & White matter $(\%)$ \\
\hline Water & 78 & 83 & 70 \\
Inorganic salts & 1.1 & 1.0 & 1.3 \\
Proteins & $8.0-10.0$ & 7.5 & 8.5 \\
Lipids: & $9.0-11.0$ & 5.8 & 14.8 \\
$\quad$ Cholesterol & $2.0-3.0$ & 1.0 & 3.9 \\
Phosphatides & $5.0-6.0$ & 3.9 & 6.5 \\
Cerebrosides & 2.0 & 0.9 & 4.4
\end{tabular}

Table 6.1: Composition of adult human brain [190]. The total protein content represents 8$10 \%$ and the total lipid content is about $10 \%$ of the total fresh weight. Lipids predominate in the brain (over $50 \%$ of the total dry weight). The difference in white and gray matter reflects the innate composition of each: myelinated fibers in white matter versus cell bodies, unmyelinated fibers and dendrites in grey matter.

In most of the earlier studies on tissue water in vivo, the relaxation curve was studied in a rather limited time domain and thus appeared to fit a single exponential decay which implies rapid exchange between the hydration water and the rest of the tissue water. This interpretation is not in agreement with the results of this study, since distinctive relaxation components, which can be associated with myelin water, bulk tissue water and hydration water, are clearly seen. The multicomponent behavior is not masked by exchange. Hence, the observation of multicomponent relaxation behavior allows discussion of the $T_{2}$ behavior in more detail than could be done when only single-component behavior was apparent. 


\subsubsection{Intracellular vs Extracellular}

It is tempting to associate the longer two relaxation components with intracellular and extracellular water. However, it is very unlikely that the relaxation behavior in these two compartments is significantly different in spite of their diverse geometrical structure. It can reasonably be assumed that both compartments have their water structured in a similar manner, as long as they share the same cellular membrane since similar hydration layers can be expected to form on both sides of the membrane. Furthermore, the intracellular and extracellular water pools are not distinct since water continuously moves across the cell membrane by various means including osmosis, active transport and diffusion.

\subsection{Diffusion Measurements}

\subsubsection{Experimental Protocol}

Numerous studies in the literature report a biexponential diffusion decay but in many of these experiments the necessary precautions have not been taken to ensure that the fibres in the voxels being studied are uniformly oriented. Consequently, the proposed interpretations of the biexponentiality of the diffusion decay presented in these works is suspect since it could simply be due to the presence of two fibre populations in the same voxel but oriented differently. With the experimental procedure used here, this problem has been effectively avoided.

Great care was taken in the slice positioning and the ROI selection to ensure that the axonal fibres in the regions studied were similar and oriented in the same direction, as much as possible. This was done by selecting areas of the brain with high $F A$ values since this can only 
be achieved if the orientations of the fibres in the voxel are consistent. The mean $F A$ for the SCC and the GCC, averaged over the 17 measurements reported here, were $0.81 \pm 0.05$ and $0.83 \pm 0.04$ respectively. It was also important to find regions where the predominant fibre orientation is well known so that the diffusion sensitizing gradients, which are defined in the laboratory frame of reference, could be oriented in a specified direction relative to the long axis of the axons, in the patient frame of reference, for the voxels being studied. Both of these constraints have been satisfied by studying the SCC and the GCC and by using the slice selection procedure described in Section 4.1.3.

The diffusion weighted images were acquired using an echo planar sequence. With this very fast sequence an image can be acquired in about $100 \mathrm{~ms}$ so that the effects of macroscopic rigid body motion during the acquisition (e.g. the subject moving his/her head) are negligible. The standard motion constraints on the head coil were also used to prevent motion. The images were retrospectively reviewed to see if there was any detectable movement between scans but none was seen.

Another important part of the protocol was the removal of the RB from the decay curves using a post-processing technique. The procedure used here has not been reported previously. Most studies in the literature either ignore the RB, with the result that their decay curves are distorted, or they do not take measurements at high $b$-values where the RB occurs, with the result that the decay is not completely measured. Correcting for the effects of RB allowed us to measure the decay reliably and undistorted over an extended range of $b$-values. This gives confidence that 1 ) the decays reported here are more reliable than many of the ones reported in the literature, 2) these decays truly characterize water diffusion in the SCC and the 
GCC and 3) the corresponding fit parameters accurately reflect the water dynamics for these tissues. The experimental results were also shown to be very reproducible.

\subsubsection{The Diffusion Decays}

The measured diffusion decays were analyzed by fitting them to a biexponential function. The obtained goodness of fit values were very close to one, indicating that the curves were well characterized by this function. The decay curves showed a very strong dependence on the orientation of the axons in the voxel. For diffusion perpendicular to the axons the signal decays very slowly and is strongly biexponential with the signal fractions for the two components being approximately equal (see Fig 5.1 and Tables 5.4 and 5.5). In this case, the RB does not have a significant effect even for the largest $b$-values studies since the SNR remained relatively large (i.e. well above the noise floor). For $b=10,000 \mathrm{~s} / \mathrm{mm}^{2}$ the signal had decayed to about $40 \%$ of its initial value. Conversely, for diffusion parallel to the axons the signal decays very quickly; the signal strength for $b=2,000 \mathrm{~s} / \mathrm{mm}^{2}$ was about $3 \%$ of its equilibrium value (after RB correction). The decay is again biexponential but it is now dominated by the fast diffusion process which accounts for about $95 \%$ of the total signal. $D_{\text {fast }}$ drops from about $1.8 \times 10^{-3} \mathrm{~mm}^{2} / \mathrm{s}$ to about $0.7 \times 10^{-3} \mathrm{~mm}^{2} / \mathrm{s}$ as $\alpha$, the angle between the axonal orientation and the diffusion measurement direction, is increased from $0^{\circ}$ to $90^{\circ}$. There is a small tendency to a similar but more subtle trend for $D_{\text {slow }}$ but to within experimental error it could be concluded that $D_{\text {slow }} \approx 0.08 \times 10^{-3} \mathrm{~mm}^{2} / \mathrm{s}$ and is constant with orientation. $D_{\text {slow }}$ appears to be consistent with values for the mobility of water in the hydration layers reported in the literature [145]. 
The measured diffusion decay changes considerably for $\alpha$ between $30^{\circ}$ and $90^{\circ}$ and more subtly for $\alpha<30^{\circ}$. These decay curves have been normalized to the signal intensity acquired with $b=0$ for improved visualization of the small differences when $\alpha<30^{\circ}$. The striking changes in the signal decay and in $D_{\text {fast }}$ with orientation are consistent with the high degree of anisotropy found in the corpus callosum. It is evident that this anisotropy partially reflects the complex diffusion process for this particular white matter region.

The anisotropic behaviour of $D_{f a s t}$ has been known for many years. In fact, it is this property of white matter that has been exploited in the development of DTI and Tractography. Plausible explanations for this behavior have been given in terms of restricted/hindered diffusion in intracellular and extracellular compartments. If the axons are simply considered to be inert cylindrical containers then the observed anisotropic diffusion makes some sense since the shape of the container, and therefore the effective diffusion distance to the container wall, changes with orientation. However, with this sort of model the signal fractions should be constant since the container sizes do not change whereas in reality the signal fractions strongly depend on orientation (see Fig. 5.7). This is very difficult to explain in terms of the traditional compartmentalization approaches that have been proposed previously in the literature.

The failure of the existing models to explain the observed signal fractions prompted us to look for a different explanation; one that could account for the observed anisotropy of both $D_{\text {fast }}$ and the signal fractions. Most of the proposed models have treated the water molecules as being inert and assumed that their dynamics were determined exclusively by elastic collisions with whatever obstacles they encountered (e.g. other water molecules, cell membranes, proteins, etc.) whereas the reality is quite different. Since water molecules are polar, they have significant electrostatic interactions with their surroundings, especially with other water 
molecules. This important property of water should be taken into account when trying to develop a model to explain the diffusion of water molecules in cells.

Although some models could account for a pseudo-biexponential [34, 41, 55], diffusion behaviour and diffusion anisotropy in white matter, they may not be validated in the brain cortex, since true restricted diffusion effects have not been really observed for water in the brain.

\subsubsection{Rician Bias Correction}

The RB correction had a significant effect on the decay when diffusion parallel to the major axis of the axons was measured while it had a negligible effect for diffusion across the axons. For diffusion parallel to the axons the noise floor was reduced by a factor of 6 . When the RB is not properly handled $D_{\text {fast }}$ is about $17 \%$ too high (see Tables 5.4 and 5.5). Uncorrected decay curves also lead to a progressively larger overestimation of the true anisotropy as the SNR decreases. Furthermore, since the shape of the decay curves changes with $b$-value, the presence of the noise floor at high $b$-values could be misinterpreted as evidence of an elevated hindrance or restriction effect.

The noise floor, which occurs in the tail of the diffusion decay curves also introduces an orientational bias to $D_{\text {slow }}$ (compare Tables 5.4a with 5.7a, for example) that is not real (see Tables 5.10 and 5.11). This adds more confusion to the interpretation of the diffusion behavior. Before RB correction, the value of $D_{\text {slow }}$ extracted from the fit for diffusion parallel

to the axons $\left(D_{\text {slow }} \approx 0.004 \times 10^{-3} \mathrm{~mm}^{2} / \mathrm{s}\right)$ is too small to be a valid diffusion coefficient for water. This suggests that the decay in this case may be biexponential; it may be inherently non-exponential. However, after RB correction there is a small signal fraction (4\% of the 
total) associated with a slowly diffusing pool that can be resolved after the noise floor has been considerably lowered. This indicates the presence of a slowly diffusing water pool which is unable to diffuse far enough to be dephased in spite of an increase in diffusion weightings.

\subsubsection{The Hydration Layers}

Water in cellular environments interacts strongly with polar regions on macromolecules such as protein side groups and phospholipid head groups (see also section 2.8.1). These water molecules form hydration layers around the cellular macromolecules and along the membrane surfaces of the cell. Due to the polar nature of water molecules the more positively charged oxygen atom will be electrostatically attracted to negatively charged side groups whereas the hydrogen atoms will be attracted to negatively charged side groups. These electrostatically bonded water molecules, which form the first hydration layer, effectively act as polar side groups themselves and induce a second, less tightly bound, hydration layer, and so on. Each hydration layer is less and less ordered as the distance from the macromolecular surface increases. The first hydration layer interacts strongly enough with the polar side groups that its $T_{2}$ is likely too short to contribute to the measured DWI signal. From the results presented in Section 5.1 and discussed in section 6.1 above, the $T_{2}$ for the first hydration layer can be taken to be about $15 \mathrm{~ms}$ with a signal fraction between 10 and $15 \%$. This means that less than $1 \%$ of the signal measured at $T E=200 \mathrm{~ms}$ comes from this water pool. The diffusion components that are attributed to water in hydration layers in this thesis are assigned to the second and higher hydration layers.

The water diffusion coefficient in the hydration layers is highly anisotropic [191]. The molecular arrangement of the first hydration layer occurs in an anisotropic manner determined 
by the preferential orientation of the hydrogen bonds with the polar side groups on the macromolecular surface. This arrangement of water dipoles near the charged macromolecular surface generates an anisotropic potential whose spatial properties remain unchanged during the reorientation of a water molecule. As a result, the anisotropic potential experienced by each water molecule is not averaged out by its rotational motion. That is, the local rotational motion about an axis perpendicular to the membrane surface has a higher rate than motions perpendicular to the membrane plane which leads to freer diffusion within a given hydration layer than between hydration layers.

The mobility of water in the first hydration layer is greatly reduced since the H-bonds between water and protein-lipid groups are more stable than the H-bonds in bulk water. The chain-like water structures stabilized by H-bonding to polar groups on the macromolecules have a lifetime of $10^{-4}$ to $10^{-6} \mathrm{~s}$ [138]. For the first hydration layer, the rotation of the water molecules is the dominant motion with relatively little translational diffusion. For the outer hydration layers the rotational degree of freedom approaches that of free bulk water but translational diffusion appears to be significantly smaller than in bulk water.

The cytoplasm typically contains up to $400 \mathrm{~g} / \mathrm{L}$ of macromolecules, which may occupy $5-40 \%$ of the total volume of the cell [192]. As a result, the cell is extremely crowded with macromolecules often being separated by only a few nanometers. Since a water molecule is about $0.3 \mathrm{~nm}$ in diameter, it can be seen that most of the water in a typical cell will be part of a hydration layer. It is, therefore, to be expected that very little of the cell's water will be completely free of the effects of these hydration layers and will diffuse as if it were in its pure liquid state. With the hydration layers tending to orient the water molecules, hydrogen bonding between water molecules is stronger here than it would be in the pure liquid. This 
causes the water to form more of a gel-like state than a pure liquid state. This structured water, which extends over several hydration layers from the membrane interface, contributes to slowing the water diffusion.

The observed diffusion coefficient in aqueous protein solutions has been shown to depend on protein concentration [145]. Recently, Colsenet et al [22] modeled water diffusion in cells by taking into account the obstruction of water by proteins and water-protein interactions (see Section 1.2). With their model they predict that the diffusion coefficient in cells will decrease as the protein concentration increases.

Although the concentration of macromolecules in the extracellular space is smaller than in the intracellular space, there will still be many membrane proteins and phospholipid head groups that will form hydration layers much as has been described above for the intracellular space. The cell membranes can be expected to have similar hydration layers on both their inner and outer surfaces. However, since the axons are tightly packed in the corpus callosum it is reasonable to expect that most of the extracellular water will be hydration water associated with membrane sidegroups. It is therefore reasonable to assume that contributions to $D_{\text {fast }}$ come partly from intracellular hydration layers and partly from extracellular hydration layers and that these contributions should correlate with the amount of water in each compartment. Water mobility in both intracellular and extracellular compartments should be similar and it seems quite unlikely that large differences in the diffusion coefficient would be obtained for these two regions. 


\subsubsection{Diffusion in the Corpus Callosum}

\subsubsection{Parallel Diffusion}

When diffusion parallel to the long axis of the axons was measured (i.e. $\alpha=0$ ), it appeared initially that a monoexponential model might be enough to characterize the decay for both the SCC and the GCC. However, after careful inspection, a small fraction $\left(f_{\text {slow }} \approx 5 \%\right)$ associated with a slowly diffusing water pool was found. This component could only be resolved after the RB correction was applied. These measurements demonstrate that approximately $95 \%$ of the axonal cell water diffuses parallel to the axons with the relatively large diffusion coefficient of about $D_{\text {fast }}=1.8 \times 10^{-3} \mathrm{~mm}^{2} / \mathrm{s}$. Considering that the diffusion coefficient of bulk water at body temperature is about $3.0 \times 10^{-3} \mathrm{~mm}^{2} / \mathrm{s}$, the axonal water can be seen to diffuse relatively freely parallel to the axons but still significantly slower than free water. This result suggests that for diffusion parallel to the axons 1) the majority of water within the hydration layers contributes to $D_{\text {fast }}$ and 2) the diffusion properties of water in this tissue differ from those in pure 'bulk' water. It also seems reasonable to assume that the contributions to $D_{\text {fast }}$ come partly from intracellular and partially from extracellular compartments and these contributions should correlate with the amount of water in these compartments.

Microimaging diffusion measurements in an isolated single Aplysia neuron reported a marked decrease in the $A D C$ for water in the cytoplasm relative to pure water [193, 194]. In spite of its large diameter $(200-300 \mu \mathrm{m})$, this giant Aplysia neuron was considered in these studies to be a valid model for mammalian neurons with $A D C$ values assigned to cytoplasmic water of the order of $0.2-0.3 \times 10^{-3} \mathrm{~mm}^{2} / \mathrm{s}$. Furthermore, these studies have implications both for modeling the NMR characteristics of water in tissue and also for understanding water- 
macromolecule interactions within cells. However, Beaulieu [195] reported diffusion coefficients of water parallel and perpendicular to the long axis of the giant squid axon, with minimal interference from membranes, at $20^{\circ} \mathrm{C}$, as being equal to $(1.61 \pm 0.06) \times 10^{-3} \mathrm{~mm}^{2} / \mathrm{s}$ and $(1.33 \pm 0.09) \times 10^{-3} \mathrm{~mm}^{2} / \mathrm{s}$, respectively, eliminating also the possibility of a significant anisotropy within the axoplasm. In terms of the hydration layer model proposed here, the water diffusion was found to be close to the free diffusion limit in this non-myelinated axon since the very small amount of protein content in the squid axon $(\sim 2.5 \%)$ is too dispersed to generate in the axoplasm a fairly firm gel-like structure.

On the other hand, $D_{\text {slow }} \approx 0.08 \times 10^{-3} \mathrm{~mm}^{2} / \mathrm{s}$ can reasonably be assigned to a small part of the hydration water probably in the second or third hydration layer. As mentioned before, the water in the first hydration layer is not expected to contribute to the measured signal when $T E=200 \mathrm{~ms}$. For hydration layers beyond the first, the water molecules are progressively more free to move leading to a longer $T_{2}$, due to increased motional averaging of dipole-dipole interactions, as well as increased translational mobility. For the outer hydration layers the rotational freedom approaches that of free bulk water but translational diffusion appears to be significantly smaller than in bulk water.

In normal biological membranes, the slow lateral diffusion of lipids and proteins, which is on the order of $10^{-6} \mathrm{~mm}^{2} / \mathrm{s}$ [196], is a determinant of the rate at which the water from the first hydration layer diffuses. Thus, membrane proteins in a sense extend the range of their influence via their hydration shells. However, as mentioned above, these spins will not have a significant contribution to the measured DWI signal. In contrast, the outer hydration layers are deemed to behave like quasifree bulk water. Measurements of diffusion in phospholipid membrane systems revealed values for the water diffusion coefficient in the hydration layers 
somewhere between $0.12 \times 10^{-3}$ and $0.40 \times 10^{-3} \mathrm{~mm}^{2} / \mathrm{s}$ depending on the water concentration per lipid-protein headgroup [145]. These values are in substantial agreement with the values reported here.

\subsubsection{Perpendicular Diffusion}

When the diffusion gradient was switched to perpendicular to the axons, a strong anisotropic response was observed in both $D_{\text {fast }}$ and $f_{\text {fast. }}$. The large drop in $D_{\text {fast }}$ (about $61 \%$ ) could be interpreted as being due to an increase in geometrical obstructions in the SCC since the axons are tightly wrapped by their myelin sheath which could be a significant barrier to the diffusion of water. However, studies in the literature apparently contradict this hypothesis since the diffusion distances reported in the literature increase well beyond cell dimensions for longer diffusion times $[197,198]$ suggesting that the water molecules diffuse fairly easily through the cell membranes. This may occur either passively or through transporters, such as the specific aquaporin channels which have been found to be abundant in the brain [29]. This implies that the membranes should be considered to be hindrances to water diffusion as opposed to restricting barriers. Similar results have been reported for cell suspensions [25].

The observed reduction of $D_{\text {fast }}$ more likely occurs both because diffusion in the hydration layers is highly anisotropic [191] and because of obstructions and compartments encountered by the water molecules during the measurement. Unfortunately, it is difficult to distinguish these effects because the relatively large voxel sizes and diffusion times required for DWI mean that the observed signal is both a temporal and spatial average of a great many diffusion pathways. The water molecules contributing to the signal will have experienced many interactions and collisions during each experiment. 
$D_{\text {slow }}$ for diffusion across the axons is about $0.08 \times 10^{-3} \mathrm{~mm}^{2} / \mathrm{s}$ and shows minimal sensitivity to the gradient orientation. This slow diffusion coefficient is consistent with the dynamic properties of the hydration layers. The diffusion coefficient measured by Trantham $e t$ al [166] using quasielastic neutron scattering, which was $\sim 10^{-5} \mathrm{~mm}^{2} / \mathrm{s}$, is also consistent with the $D_{\text {slow }}$ values reported here. As mentioned above, the diffusion coefficient for water in the hydration layers is expected to be anisotropic. However, there is a subtle trend towards anisotropy in our data but it is not conclusive beyond experimental error.

Such slow diffusion between hydration layers suggests that these hydration layers of relatively static fluid close to the membrane could be the rate-limiting factor for water transport across the membrane. Wassal [145] showed that slow water diffusion across phospholipid membrane bilayers was consistent with the water permeability coefficient, $P$, reported for egg phosphatidylcholine [199] which is on the order of $1-10 \mu \mathrm{m} / \mathrm{s}$ and corresponds to a very small diffusion coefficient of about $D=10^{-7} \mathrm{~mm}^{2} / \mathrm{s}$ for a $10 \mu \mathrm{m}$ thick bilayer. Fenichel and Horowitz [134] suggest that membrane permeability and diffusion across the membrane is limited more by the cytoplasm rather than by the membranes themselves. Their values calculated for the diffusion coefficients, are also of the order of $10^{-7} \mathrm{~mm}^{2} / \mathrm{s}$. Experiments have also shown that increasing the length of protein hydrophilic chains decreases the permeability of membranes [200], suggesting that the larger amount of hydration associated with the extra length of protein may pose extra limitations to the water transport across membranes. Finer [131] proposed that each hydration layer is completely filled before population of the next, less strongly bound layer arises and that the motion of all water molecules within a given layer is equivalent. This model provides a different explanation for why diffusion of water from one hydration layer to another appears to be limited. 
The $D_{\text {slow }}$ values compare favorably with the values measured by Grant et al [201] in an isolated single Aplysia neuron; the average $A D C$ s from the cytoplasm (with relative fractions) were $(0.48 \pm 0.14) \times 10^{-3} \mathrm{~mm}^{2} / \mathrm{s}(61 \pm 11 \%)$ and $(0.034 \pm 0.017) \times 10^{-3} \mathrm{~mm}^{2} / \mathrm{s}(32 \pm$ $11 \%)$. They proposed that these components are a result of the subcellular structure of the cytoplasm, transport mechanisms or water complexes with larger, protein molecules.

Callaghan et al [202] found that the diffusion coefficients of water parallel and perpendicular to a potassium palmitate bilayer sample were $D_{\|}=1.7 \times 10^{-3} \mathrm{~mm}^{2} / \mathrm{s}$ and $D_{\perp}=$ $0.05 \times 10^{-3} \mathrm{~mm}^{2} / \mathrm{s}$, respectively, while negligible diffusion perpendicular to a phosphatidylcholine lipid-bilayer surface was detected by Volke et al [203]. Rudakova et al [204] reported $D_{\|}=0.57 \times 10^{-3} \mathrm{~mm}^{2} / \mathrm{s}$ and $D_{\perp}=0.05 \times 10^{-3} \mathrm{~mm}^{2} / \mathrm{s}$ for an oriented system of stacked membranes. She mainly investigated the transport of water molecules across lipid bilayers observing a decrease in the coefficient of permeability (diffusion transverse to bilayers) as the concentration of sterols (cholesterol and ergosterol) were increased from 10 to $30 \%$ mol. Low values for diffusion perpendicular to stacked membranes agree also with the observations of Beaulieu [54] who reported $D_{\perp}=(0.18 \pm 0.03) \times 10^{-3} \mathrm{~mm}^{2} / \mathrm{s}$ for the olfactory nerve and $D_{\perp}=(0.20 \pm 0.06) \times 10^{-3} \mathrm{~mm}^{2} / \mathrm{s}$ for the optic nerve. Although the plasma membrane of these axons was regarded by Beaulieu to be the primary determinant of water diffusion restriction, the reported diffusion coefficients appears to be consistent with the diffusion coefficients of water molecules in hydration layers. Furthermore, their slightly elevated values are consistent with the fact that the olfactory nerve is completely unmyelinated and the optic nerve is not heavily myelinated [54]. 


\subsubsection{Anisotropy}

The measured diffusion decay changes considerably for $\alpha$ between $30^{\circ}$ and $90^{\circ}$ and more subtly for $\alpha<30^{\circ}$. $D_{\text {fast }}$ also shows a large drop, by about $63 \%$, as $\alpha$ is increased from $0^{\circ}$ to $90^{\circ}$ (see Fig. 5.5). This drop can be explained in terms of the anisotropy of hydration water as has been discussed at length in the last two subsections. The signal fractions $f_{\text {fast }}$ and $f_{\text {slow }}$ are also anisotropic, changing from $95 \%$ and $5 \%$, respectively when $\alpha=0^{\circ}$ to being nearly equal when $\alpha=90^{\circ}$ (see Fig. 5.7). This anisotropic effect has been observed previously in human brain by Clark et al [35] as the fast diffusion fraction in white matter varies between $90 \%$ and $50 \%$ from a measurement direction parallel to the fibers to a direction perpendicular to them. These findings are in good agreement with the results reported here. A nonsignificant trend was also observed for $D_{\text {slow }}$ with increasing $\alpha$ (see Table 5.11). The striking changes in the signal decay and in the fit parameters with orientation are consistent with the high degree of anisotropy found in the corpus callosum and an increase in geometrical obstructions for diffusion across the axons since the high packing density of the axons and myelin membranes and their associated hydration layers will hinder water mobility. It is evident that this anisotropy partially reflects the complex diffusion process for this particular white matter region.

The observed anisotropy of the signal fractions $f_{f a s t}$ and $f_{\text {slow }}$ can be explained if the water in the outer hydration shells diffuses more freely parallel to the axons than perpendicular to them. Support for this suggestion can be found in the literature and is reviewed in the last paragraph of Section 6.2.4.2. In this case, the outer hydration layers behave as "free" water for $\alpha=0^{\circ}$ and contribute to $f_{\text {fast }}$ and they behave as "bound" water for $\alpha=90^{\circ}$ and contribute to $f_{\text {slow. }}$ The inner hydration water is considered to contribute to $f_{\text {slow }}$ for all orientations, 
consistent with the observation that $D_{\text {slow }}$ changes very little with $\alpha$. The residence times of water molecules at specific hydration sites seem to depend almost exclusively on the geometrical arrangement of the polar water molecules in adjacent hydration layers [130]. Such an arrangement would be expected to hinder diffusion between hydration layers more than diffusion within hydration layers leading to diffusional anisotropy for the hydration water. Hence, there will be an apparent increase in $f_{\text {slow }}$ for measurements across the axons relative to measurements parallel to the axons. This suggestion is consistent with the observation that the membrane hydration layers appear to form a barrier to water diffusion perpendicular to the membranes.

The water associated with $f_{\text {fast }}$ and $f_{\text {slow }}$, should be considered to be in different functional phases as opposed to different physical compartments (e.g. intracellular and extracellular). It should be noted that a distribution of cell size or cell density within a given voxel will result in a corresponding distribution of the relative amount of hydration water.

The mean spin displacements, predicted by Einstein's equation (Eq. 1.1) for diffusion in one dimension with the $D_{\text {fast }}$ values given in Table 5.10 for the SCC and $t_{d}=47 \mathrm{~ms}$, are about $13 \mu \mathrm{m}$ and $12 \mu \mathrm{m}$ for $\alpha=0^{\circ}$ and $\alpha=30^{\circ}$, respectively. This is incompatible with the traditional restricted diffusion point of view since for $\alpha=30^{\circ}$ and $t_{d}=47 \mathrm{~ms}$ there should be a high effect of compartmental obstruction in the $1.5 \mu \mathrm{m}$ diameter axons found in the SCC.

The investigations presented here have much in common with most of the previous diffusion studies that have attempted to explain why water diffusion is anisotropic in white matter. But it definitely goes against the idea that the axonal plasma membrane and the membranes in the myelin sheath are the primary sources of this observed anisotropic 
behaviour. Rather, we believe that the hydration layers associated with these membranes have the most important effect.

Myelin is not required to generate tissue anisotropy [54] but its presence may modulate the degree of anisotropy since an increase in myelin content will induce more hydration water. In turn, the absence of myelin will alter the absolute $A D C$ values. If that is the case, then anisotropic water diffusion in neural fibres should not be regarded as myelin specific. Moreover, anisotropy increases with normal brain development [205] although there are questions as to whether this can be associated with increasing levels of myelination or just an improved coherence of the fibre tracts. Pierpaoli et al [53] also had difficulty in showing a significant correlation between the observed $F A$ values in various white matter tracts with specific tissue microstructures in the adult human brain.

The quantitative or even qualitative determination of the importance of myelin, relative to the axonal plasma membrane, for anisotropy in a given axonal fiber has not yet been resolved. A direct assessment of anisotropy between distinctive fibers with different axonal diameters, degrees of myelination, and fibre packing densities is difficult. Processes related to 1) demyelination or primary mechanical disruptions of axons that lead to a loss of continuity of the myelin sheath or 2) secondary conditions such as the presence of edema or necrotic tissue, can cause reduced anisotropy in white matter. Measurements of this change in anisotropy can reflect, with great sensitivity, the subtle water movements and restructuring associated with these pathological conditions.

The importance of the observed subtle decrease in $D_{\text {slow }}$ as $\alpha$ is increased is unclear since it is barely beyond experimental error. However, such a dependence would be consistent with the picture proposed here since an increase in $f_{\text {slow }}$ would mean an increase in the amount 
of water in the inner hydration layers which would cause the associated average diffusion coefficient to decrease.

The observed monotonic increase in $f_{\text {slow }}$ and the $63 \%$ decrease in $D_{\text {fast }}$ with increasing $\alpha$ both add credibility to the proposed model and are quite consistent with the suggested diffusion anisotropy of the hydration layers. Water molecules are considered to diffuse more readily within a given hydration layer than they do between layers. This concept is supported by various reports in the literature which are reviewed at the end Section 6.2.4.2

There is no doubt that this is an oversimplified picture of the real situation for water in brain tissue; it is not possible to rigorously include all properties of the tissue. Certainly, some water molecules in the hydration layers have dynamics very different from those in the bulk, but there is a continuum between these "bound" molecules and those that behave dynamically as though they are indeed in the bulk fluid. The bound water described here is characterized by a single overall mobility but may well not be of a single structural type and the first hydration layer, treated separately, is the most stable structure where the water is relatively immobile. This stability is provided both by the strength of the interaction and by the great inertia of the macromolecular components.

\subsubsection{Consistency: GCC vs SCC}

In spite of the anatomical similarities between the GCC and the SCC there is also a significant difference in $f_{\text {slow }}$ and $f_{\text {fast }}$ for $\alpha=90^{\circ}$ while the values for both $D_{\text {fast }}$ and $D_{\text {slow }}$ stay remarkably close. This adds credibility to the proposed model since a small deviation in the axon diameter would be expected to induce a change in $f_{\text {slow }}$ which should scale with the membrane surface area. 
The difference in the diffusion pattern between the GCC and the SCC correlates with differences in water content, water-free chemical composition, the number of macromolecules and metabolic activity in these callosal areas. According to neuroanatomical tracing studies [206], the regions of the corpus callosum connecting areas of higher order cognitive processing in the temporal and parietal lobes are the anterior and mid SCC. Consequently, the SCC tends to have more heavily myelinated, thicker fibres. The number of fibers per unit area is highest in the GCC and slow-conducting, thinly myelinated fibers, less than $1.5 \mu \mathrm{m}$ in diameter, are more abundant in this area. The more heavily myelinated posterior part of the corpus callosum may account for a higher speed of nerve transmission and a more efficient neural network. More relevant to the current work is that an increase in axonal cross sectional area correlates with a decrease in fiber density due to an increased fiber diameter. Some authors have reported that the most common axon diameter is $0.6 \mu \mathrm{m}$ in the genu with a steady increase in the posterior direction to reach $1.0 \mu \mathrm{m}$ in the splenium [120]. 


\section{Chapter 7}

\section{Conclusions}

The goal of this thesis was to propose a novel explanation for the observed diffusion properties of tissue water in human white matter in vivo by considering the electrostatic interactions of water molecules with each other and with their surroundings. These interactions lead to the formation of hydration layers around macromolecules and along membrane surfaces and cause the water in the cell to behave more like a gel that a liquid. Incorporation of these considerations into the analysis presented in this thesis, has allowed to develop novel explanations for: 1) why the measured fast diffusion component is smaller than the diffusion coefficient of free water, 2) why it is anisotropic, 3) the observed signal fraction values and 4) the anisotropy of the signal fractions.

The experimental results presented in this thesis are mainly from a set of carefully designed diffusion decay measurements in two regions of the corpus callosum of healthy 
human volunteers in vivo. The experimental results are explained using a hydration layer model rather than the more traditional intracellular/extracellular restricted diffusion approach. Supporting $T_{2}$ decay experiments are also presented and analyzed in terms of the same hydration layer model. At large $b$-values some of these decays were affected by Rician bias and a correction for this was required. In Chapter 3 a novel Rician bias correction technique is explained and then extended to a more accurate and efficient algorithm which was then used to remove this bias from the measured diffusion decays.

Traditionally, diffusion in white matter has been interpreted in terms of restricted diffusion models with two well defined compartments: the intracellular space and the extracellular space. With these models the water molecules are normally considered to diffuse as if they were in pure water until they meet a barrier, such as the cell membrane, which simply reflects the particle back in a very classical sense. However, these models have not been successful in accounting for all of the observed results; in particular, the observed signal fractions are inconsistent with the predictions of these models.

Water in cellular environments interacts strongly with polar regions on macromolecules in the tissue and form hydration layers around the cellular macromolecules and along the membrane surfaces of the cell. Due to the polar nature of water molecules the more positively charged oxygen atom will be electrostatically attracted to negatively charged side groups whereas the hydrogen atoms will be attracted to positively charged side groups. These electrostatically bonded water molecules, which form the first hydration layer, effectively act as polar side groups themselves and induce a second, less tightly bound, hydration layer, and so on. Each hydration layer is less and less ordered as the distance from the macromolecular surface increases. 
Water diffusion in the hydration layers is highly anisotropic [196]. The molecular arrangement of the first hydration layer occurs in an anisotropic manner determined by the preferential orientation of the hydrogen bonds with the polar side groups on the macromolecular surface. This arrangement of water dipoles near the charged surface generates an anisotropic potential whose spatial properties remain unchanged during the reorientation of a water molecule. As a result, the anisotropic potential experienced by each water molecule is not averaged out by its rotational motion. This suggests that water molecules are freer to diffuse within a given hydration layer than between them.

Evaluation of water diffusion in the corpus callosum unambiguously revealed fast and slow diffusion components corresponding to two distinct water pools. With the interpretation put forward in this thesis one of these pools consists of water which is close enough to macromolecular or membrane surfaces that its diffusional motion is affected by interactions between the polar water molecules and the charged macromolecular sidegroups (i.e. the hydration layers) and the water in the other pool is far enough from this surface that it preserves its bulk state properties.

By considering the effect of hydration layers on the diffusion of water in tissue we have been able to propose a more satisfactory explanation for the experimental observations reported here and in the literature. 


\section{References}

[1] Mori S, Barker P B. Diffusion Magnetic Resonance Imaging: Its principle and applications; 1999. The Anat Record; $257: 102-109$.

[2] LeBihan D, Breton E, Lallemand D, Grenier P, Cabanis E, Laval-Jeantet M, 1986. MR imaging of intravoxel incoherent motions: application to diffusion and perfusion in neurologic disorders. Radiology; 161: 401-407.

[3] Anderson A W, Gore J, 1994. Analysis and correction of motion artifacts in diffusion weighted imaging. Magn. Reson.Med; 32: 379-384.

[4] Einstein A, 1956. (collection of papers translated from the German) Investigations on the Theory of Brownian Motioned R Furthe and A D Cowper (New York: Dover).

[5] LeBihan D, 2003. Looking into the functional architecture of the brain with diffusion MRI Nat. Rev. Neurosci, 4; 469-80.

[6] Tanner J E, 1978. Transient diffusion in a system partitioned by permeable barriers: application to NMR measurements with a pulsed field gradient $J$. Chem. Phys; 69:1748-54.

[7] Tanner J E, 1979. Self diffusion of water in frog muscle Biophys. J; 28: 107-16. 
[8] Cooper R L, Chang D B, Young A C, Martin J and Ancker-Johnson B 1974. Restricted diffusion in biophysical systems Biophys. J; 14:161-77.

[9] Moseley M E, Cohen Y and Mintorovitch J, 1990b. Early detection of regional cerebral ischemic injury in cats: evaluation of diffusion and T2-weighted MRI and spectroscopy Magn. Reson. Med; 14: 330-46.

[10] Moseley M E, Cohen Y and Kucharczyk J, 1990a. Diffusion-weighted MR imaging of anisotropic water diffusion in cat central nervous system Radiology; 176: 439-46.

[11] Clark C A, Barker G J, Tofts P S, 2000. Diffusion tensor MRI of the spinal cord. Magn Reson Med; 44: 884-892.

[12] Beaullieu C, Does M D, Snyder R E, Allen P, 1996. Changes in water diffusion due to wallerian degeneration in peripheral nerve. Magn Reson Med; 36: 627-635.

[13] Skorpil M, Engstrom M, Nordell A, 2007. Diffusion direction dependent imaging: A novel MRI approach for peripheral nerve imaging. Magn Reson Imaging; 25: 406-411.

[14] Chenevert T L, Brunberg J A, Pipe J G, 1990. Anisotropic diffusion in human white matter: demonstration with MR techniques in vivo. Radiology; 177: 401-405.

[15] Douek P, Turner R, Pekar J, Patronas N J and LeBihan D, 1991. MR color mapping of myelin fiber orientation J. Comput. Assist. Tomogr; 15; 923-9.

[16] Basser P J, Mattiello J and LeBihan D, 1994. MR diffusion tensor spectroscopy and imaging Biophys. J; 66: 259-67.

[17] Maltsev N A, 1967. Proton relaxation in some protein solutions and gels. Molec Biol; 4: $451-462$.

[18] Hansen J R, 1971. Pulsed NMR study of water mobility in muscle and brain tissue. Biochim Biophys Acta, 230: 482-486. 
[19] Hazlewood C F, Rorschach H E and Lin C, 1991. Diffusion of water in tissues and MRI Magn. Reson. Med; 19: 214-6.

[20] Rorschach H E, Chang D C, Hazlewood C F and Nichols B L, 1973. The diffusion of water in striated muscle. Ann. NY Acad. Sci; 204: 445-52.

[21] Chang D C, Rorschach H E, Nichols B L and Hazlewood C F, 1973. Implications of diffusion coefficient measurements for the structure of cellular water Ann. NY Acad. Sci; 204: $434-43$.

[22] Colsenet R, Mariette F and Cambert M, 2005. NMR relaxation and water self-diffusion studies in whey protein solutions and gels J. Agric. Food. Chem; 53: 6784-90.

[23] Cooper R L, Chang D B, Young A C, Martin J and Ancker-Johnson B, 1974. Restricted diffusion in biophysical systems Biophys. $J ; 14: 161-77$.

[24] Latour L, Svoboda K, Mitra P P and Sotak C H, 1994b. Time-dependent diffusion of water in a biological model system Proc. Natl Acad. Sci; 91: 1229-33.

[25] Garcia-Perez A I, Lopez-Beltran E A, Kluner P, Luque J, Ballesteros P and Cerdan S, 1999. Molecular crowding and viscosity as determinants of translational diffusion of metabolites in subcellular organelles. Arch. Biochem.Biophys; 362: 329-38.

[26] Assaf Y, Cohen Y, 1998. Non-mono-exponential attenuation of water and $N$-acetyl aspartate signals due to diffusion in brain tissue J. Magn. Reson; 131: 69-85.

[27] Norris D G, Niendorf T, Leibfritz D, 1994. Healthy and infarcted brain tissues studied at short diffusion times: the origins of apparent restriction and the reduction in apparent diffusion coefficient NMR Biomed; 7: 304-10.

[28] Nossin-Manor R, Duvdevani R, Cohen Y, 2005. Effect of experimental parameters on high b-value $q$-space MR images of excised rat spinal cord. Magn Reson Med 54: 96-104. 
[29] Amiry-Moghaddam M, Ottersen O P, 2003. The molecular basis of water transport in the brain. Nat. Rev. Neurosci; 4: 991-1001.

[30] Szafer A, Zhong J, Gore J C 1995. Theoretical Model for Water Diffusion in Tissues. Magn. Reson. Med; 33: 697-704.

[31] Niendorf T, Dijkhuizen R M, Norris D G, Van Lookeren Campagne M, Nicolay K, 1996. Biexponential diffusion attenuation in various states of brain tissue: implications for diffusionweighted imaging Magn. Reson. Med; 36: 847-57.

[32] Assaf Y, Cohen Y, 2000. Assignment of the Water Slow Diffusing Component in CNS Using $q$-Space Diffusion MRS: Implications for Fiber Tract Imaging. Magn. Reson. Med; 43: 191-199.

[33] Pfeuffer J, Dreher W, Sykova E, Leibfritz D, 1998. Water signal attenuation in diffusion weighted 1H NMR experiments during cerebral ischemia: influence of intracellular restrictions, extracellular tortuosity and exchange. Magn Reson Imag; 16: 1023-1032.

[34] Karger J, Pfeifer H, Heink W, 1988. Principles and application of self-diffusion measurements by nuclear magnetic resonance Adv. Magn. Reson; 12:1-89.

[35] Clark C A, LeBihan D, 2000. Water diffusion compartmentation and anisotropy at high $b$ values in the human brain Magn. Reson. Med; 44: 852-59.

[36] Maier S E, Bogner P, Bajzik G, Mamata H, Mamata Y, Repa I, Jolesz F A, Mulkern R V, 2001. Normal brain and brain tumor: multicomponent apparent diffusion coefficient line scan imaging. Radiology; 219: 842-49.

[37] Mulkern R V, Gudbjartsson H., Westin C F, 1999. Multi-component apparent diffusion coefficients in human brain NMR Biol; 12: 51-62. 
[38] LeBihan D, Urayama S, Aso T, Hanakawa T, Fukuyama H, 2006. Direct and fast detection of neuronal activation in the human brain with diffusion MRI Proc. Natl Acad. Sci. 103: 8263-68.

[39] Sykova E, Nicholson C, 2008. Diffusion in brain extracellular space. Physiol Rev; 88(14): $1277-340$.

[40] Maier S, Vajapeyam S, Mamata H, Westin C, Jolesz F A, Mulkern R V, 2004. Biexponential diffusion tensor analysis of human diffusion data. Magn Reson Med; 51: 321330.

[41] Stanisz G J, Szafer A, Wright G A, Henkelman R M, 1997. An analytical model of restricted diffusion in bovine optic nerve Magn. Reson. Med; 37: 103-11.

[42] Chin C L, Wehrli F W, Fan Y L, Hwang S N, Schwartz E D, Nissanov J, Hackney D B, 2004. Assessment of axonal fiber tract architecture in excised rat spinal cord by localized NMR q-space imaging: simulations and experimental studies Magn. Reson. Med; 52: 733-40. [43] Sehy J V, Ackerman J H, Neil J, 2002b. Evidence that both fast and slow water ADC components arise from intracellular space Magn. Reson. Med; 48: 765-70.

[44] Schwarcz A, Bogner P, Meric P, Correze J, Berente Z, Pal J, Gallyas F, Doczi T, Gillet B, Beloeil J, 2004. The existence of biexponential signal decay in magnetic resonance diffusionweighted imaging appears to be independent of compartmentalization. Magn Reson Med; 51: 278-285.

[45] Anderson A, Xie J, Pizzona J, Broner R, Gore J, 2000. Effects of cell volume fraction changes on apparent diffusion in human cells. Magn. Reson. Imag; 18: 689-695.

[46] Cohen Y, Assaf Y, 2002. High $b$-value q-space analyzed diffusion-weighted MRS and MRI in neuronal tissues-a technical review NMR Biomed; 15: 516-42. 
[47] Pfeuffer J, Provencher S W, Gruetter R, 1999. Water Diffusion in Rat Brain in Vivo as Detected at Very Large $b$-Values is Multicompartmental. MAGMA; 2: 98-108.

[48] Olariu E, 2003. Monte Carlo Studies of the Magnetic Resonance Diffusion Decay, MSc Thesis, Carleton University, Phys Dept.

[49] Kiselev V G, Il'yasov KA, 2007. Is the "biexponential diffusion" biexponential? Magn Reson Med; 57: 464-469.

[50] Sustanskii A L, Yablonskiy D A, Ackerman J H, 2004. Effects of permeable boundaries on the diffusion-attenuated MR signal: insights from a one-dimensional model J. Magn. Reson; 170: 56-66.

[51] Peled S, Cory D G, Raymond S A, Kirschner D A, Jolesz F A, 1999. Water diffusion, T2, and compartmentation in frog sciatic nerve. Magn Reson Med; 42: 911-918.

[52] Moseley M E , Kucharczyk J, Asgari H S, Norman D, 1991. Anisotropy in diffusionweighted MRI. Magn. Reson. Med; 19: 321-326.

[53] Pierpaoli, C, Basser P J, 1996. Toward a Quantitative Assessment of Diffusion Anisotropy. Magn. Reson. Med; 36: 893-906.

[54] Beaulieu C, Allen P, 1994. Determinants of anisotropic water diffusion in nerves. Magn Reson. Med; 31: 394-400.

[55] Assaf Y, Basser P J, 2005. Composite hindered and restricted model of diffusion (CHARMED) MR imaging of the human brain. Neurolmage; 27: 48-58.

[56] Yablonskiy D A, Bretthorst G L, Ackerman J H, 2003. Statistical model for diffusion attenuated MR signal Magn.Reson. Med; 50: 664-9. 
[57] Bennett K, Schmainda K, Bennet R, Rowe D, Lu H, Hyde J, 2003. Characterization of continuously distributed cortical water diffusion rates with a stretched-exponential model. Magn Reson Med; 50: 727-734.

[58] Liu C, Bammer R, Kim D, Moseley ME, 2004. Self-navigated interleaved spiral (SNAILS): application to high-resolution diffusion tensor imaging. Magn Reson Med; 52: 1388-1396.

[59] Ozarslan E, Shepherd T M, Vemuri B C, Blackband S J, Mareci T H, 2006. Resolution of complex tissue microarchitecture using the diffusion orientation transform (DOT). Neuroimage; 31: 1086-103.

[60] Callaghan P T 1991. Principles of Nuclear Magnetic Resonance. Microscopy Oxford Univ Press, Oxford/New York.

[61] Jensen J H, Hlpern J A, Ramani A, Lu H, Kaczynski K, 2005. Diffusional kurtosis imaging: the quantification of non-gaussian water diffusion by means of magnetic resonance imaging. Magn Reson Med; 53:1432-1440.

[62] LeBihan D, 2007. The 'wet mind': water and functional neuroimaging Phys. Med. Biol. 52: R57-R90.

[63] Jacobson B, Anderson W A, Arnold T, 1954. A proton magnetic resonance study of the hydration of deoxyribonucleic acid. Nature Lond; 173: 772-773.

[64] Berendsen H J C, 1962. Nuclear magnetic resonance study of collagen hydration. J. chem. Phys; 36: 3297-3305.

[65] Berendsen H J C, Migchelsen, C, 1965. Hydration structure of fibrous macromolecules. Ann. NY Acad. Sci; 125: 365-379.

[66] Inoue Y, 1966; J. Med. Sci. 12: 107. 
[67] Chapman G, McLauchlan K A, 1967. Oriented water in the sciatic nerve of rabbit. Nature, Lond; 215: 391-392.

[68] Ramanchandran G N, Chadrasekharan R, 1968. Interchain hydrogen bonds via bound water molecules in the collagen triple helix. Biopolymers; 6: 1649-1658.

[69] Peto S, Gillis P, Henri V P, 1990. Structure and dynamics of water in tendon from NMR relaxation measurements. Biophys $J ;$ 57: 71-84.

[70] Wang J, 1954.Theory of self-diffusion of water in protein solution. J. Am. Chem. Soc. 76: 4755.

[71] Abetsedarskaya LA, Miftakhutdinova FG, Fedotov VD,1968. State of water in living tissues. Biophysics; 13: 750.

[72] Watanabe T, Ohtsuka A, 1993. Diffusional behavior of water in polysaccharide gels by PFGSTE NMR. Mol. EnViron. IntraExtracell. Sodium, 77-80.

[73] Hills B P, Godward J, Manning C E, Biechlin J L Wright K M, 1998. Microstructural characterization of starch systems by NMR relaxation and Q-SPACE microscopy. Magn. Reson. Imaging; 16: 557-564.

[74] Brown W, Stilbs P. Self-diffusion measurements on bovine serum albumin solutions and gels using a pulsed gradient spin-echo NMR technique. Chem. Scr., 19, 161-163.

[75] Kelly S M, Butler J P, Macklem P T, 1995. Control of cell volume in oocytes and eggs from Xenopus laevis. Comp Biochem Physiol; 111A: 681-691.

[76] Glaubitz C, Burnett I J, Grobner G, Mason A J, Watts A.1999.Deuterium-MAS NMR spectroscopy on oriented membrane proteins: applications to photointermediates of bacteriorhodopsin. J Am. Chem. Soc; 121: 5787-5794. 
[77] Inglefield P T, Lindblom K A, Gottlieb A M, 1976. Water binding and mobility in phosphatidylcholine/cholesterol/water lamellar phase.Biochim. Biophys. Acta; 419: 196-205.

[78] Chien M, Smith B A, Samulski E T, Wade C G, 1974. Diffusion in oriented lamellar phases by pulsed NMR. In Liquid Crystals and Ordered Fluids, Vol 2. J. F. Johnson and R. S. Porter, editors. Plenum Press, London, pp: 67-71.

[79] Bloch F, Hansen W, Packard M E, 1946. Nuclear Induction. Phys. Rev; 69:127.

[80] Purcell E M, Torrey H C, Pound R V, 1946. Resonance Absorption by Nuclear Magnetic Moments in a Solid. Phys. Rev; 69: 37.

[81] French A P, Taylor E F, 1978. An Introduction to Quantum Physics. W.W. Norton \& Company, Inc. New York, N.Y.

[82] Reif F, 1965. Fundamentals of Statistical and Thermal Physics (McGraw-Hill Book Company, Toronto), pp: 251-488.

[83] Abraham A, 1961. The Principles and Nuclear Magnetism. Oxford University Press, London.

[84] Price W S, 1997. Pulsed-Field Gradient Nuclear Magnetic Resonance as a Tool for Studying Translational Diffusion. Basic Theory, John Wiley \& Sons Inc. Concepts Magn. Reson; 9: 299-336.

[85] Bhadelia R A, Bogdan A R, Kaplan R F, Wolpert S M, 1997. Cerebrospinal fluid pulsation amplitude and its quantitative relationship to cerebral flow pulsations: a phasecontrast MR flow imaging study.Neuroradiology; 39: 258-264.

[86] Stejskal E O, Tanner J E, 1965. Spin Diffusion Measurements: Spin Echoes in the Presence of a Time-Dependent Field Gradient. J. Chem. Phys; 42:288-292. 
[87] Tanner J E, 1967. The use of the stimulated echo in NMR diffusion studies. J Chem Phys $190 ; 52: 2523-2526$.

[88] Torrey H C, 1956. Bloch equations with diffusion terms. Phys. Rev; 104: 563.

[89] Slichter C P, 1980. Principles of Magnetic Resonance. Springer-Verlag.

[90] Gordon S Rule, T K Hitchens, 2005. Fundamentals of protein NMR Spectroscopy. Springer.

[91] Morris P G, 1986. Nuclear Magnetic Resonance Imaging in Medicine and Biology, Oxford, Clarendon.

[92] Eisenberg D, Kauzmann W, 1969. The structure and properties of water. Oxford University Press, New York.

[93] Outhred R K, George E P, 1973. A nuclear magnetic resonance study of hydrated systems using the frequency dependence of the relaxation processes. Biophys $J ; 13: 83$.

[94] Marchi M, Sterpone F, Ceccarelli M, 2002 Water rotational relaxation and diffusion in hydrated lysozyme. J.Am. Chem Soc; 124: 6787.

[95] Caputa K, Hennel, J W, Szczepkowski, T W, 1967. In: Proc. Int. Conf. on Magnetic Resonance and Relaxation, Ed. Blinc, R. North Holland Publ. Co., Amsterdam, pp: 28.

[96] Daskiewicz O K, Hennel J W, Lubas B, Szczepkowski, T. W 1963. Proton magnetic relaxation and protein hydration. Nature; 200: 1006.

[97] Oncley J L, 1943. In Proteins, Amino Acids and Peptides, Eds. Cohn, E. J. and Edsall, J. T. Hafner Publ. Co., New York and London, pp: 543.

[98] Cope F W, 1969. Nuclear magnetic resonance evidence using D20 for structured water in muscle and brain. Biophys. J; 9: 303-319. 
[99] Hazlewood C F, Chang D C, Nichols B L, Wossner D E, 1974b. Nuclear magnetic resonance transverse relaxation times of water protons in skeletal muscle. Biophys. J; 14: 583606.

[100] Fritz O G, Swift T J, 1967. The state of water in polarized and depolarized frog nerves. A proton magnetic resonance study. Biophys. J; 7: 675-686.

[101] Odeblad E, Bhar B N, Lindstrom G, 1956. Proton magnetic resonance of human red blood cells in heavy water-exchange experiments. Arch. Biochem. Biophys; 63: 221-225.

[102] Sussman M V, Chin L, 1966. Liquid water in frozen tissue: Study by nuclear magnetic resonance. Science; $151: 324$.

[103] Bratton C B, Hopkins A L, Weinberg J L, 1965. Nuclear Magnetic Resonance of Living Muscle, Science; 147: 738-739.

[104] Meiboom S, Gill D, 1958. Modified spin-echo method for measuring nuclear relaxation times. Rev Sci Intrum; 29: 688-691.

[105] Hahn EL, 1950. Spin echoes. Phys Rev; 80: 580-594.

[106] Peemoeller H, Pintar M M, 1979. Nuclear magnetic resonance multi-window analysis of proton local fields and magnetization distribution in natural and deuterated mouse muscle. Biophys $J ; 28: 339-356$.

[107] Menon R S, Rusinko M S, Allen P S, 1992. Proton relaxation studies of water compartmentalization in a model of neurological system. Magn Reson Med; 28: 264-274.

[108] English A E, Joy M L G, Henkelman R M, 1991. Pulsed NMR relaxometry of striated muscle .bers. Magn Reson Med; 21: 264-281.

[109] Cole W C, LeBlanc A D, Jhingran S G, 1993. The origin of biexponential T2 relaxation in muscle water. Magn Reson Med; 29:19-24. 
[110] Melhem E R, Mori S, Mukundan G, Kraut M A, Pomper M G, van Zijl PC, 2002 Diffusion tensor MR imaging of the brain and white matter tractography. Am J Roentgenol; 178: 3-6.

[111] Pierpaoli C, Jezzard P, Basser P J, Barnett A, Di Chiro G, 1996. Diffusion tensor MR imaging of the human brain. Radiology; 201: 637-648.

[112] LeBihan D, Mangin J F, Poupon C, Clark C A, Pappata S, Molko N, 2001. Diffusion tensor imaging: concepts and applications J. Magn. Reson. Imag; 13: 534-46.

[113] Basser P.J, Pierpaoli, C. 1996.Microstructural and physiological features of tissues elucidated by quantitative-diffusion-tensor MRI. J. Magn. Reson B, 111: 209-219.

[114] Peters A, Palay S L, Webster H D, 1991. The fine structure of the nervous system, $3^{\text {rd }}$ ed, Oxford, New York.

[115] Hajnal J V, Doran M., Hall A S, 1991. MR Imaging of anisotropically restricted diffusion of water system: technical, anatomic and pathologic considerations. J. Comput. Ass. Tomog; 15: 1-18.

[116] Morell P, Quarles R H, Norton W T, 1994. Myelin formation, structure, and biochemistry. In: Siegel GJ, ed. Basic Neurochemistry: Molecular, Cellular and Medical Aspects. 5th ed. New York: Raven Press; pp:117-143.

[117] Brady S T, Witt A S, Kirkpatrick L L, 1999. Formation of compact myelin is required for maturation of the axonal cytoskeleton. $J$ Neuroscience; 19: 7278-7288.

[118] Vandenheuvel F A, 1965. Structural studies of biological membranes: the structure of myelin. Ann. N. Y. Acad. Sci; 122: 57-76.

[119] Tomasch. J, 1954. Size, distribution and number of fibres in the human corpus callosum. Anat. Rec; 119: 119-135. 
[120] Aboitiz F, Scheibel A B, Fisher R B, Zaidel E, 1992. Fiber composition of the human corpus callosum. Brain Research; 2:143-153.

[121] Clegg J S, 1978. Hydration-dependent metabolic transitions and the state of cellular water in Artemia cysts. In: Crowe JH, Clegg JS, editors Dry Biological Systems. New York Acad Press.

[122] Clegg J S, 1984b. Intracellular water and the cytomatrix: some methods of study and current views J. Cell Biol; 99: 167s-71s.

[123] Chaplin M F, 2000.Water; its importance to life. Biophys. Chem; 83: 221.

[124] Finney J L 2004. Water? What's so special about it? Phil. Trans. R. B; 359: 1145-63.

[125] Baker E N, 1995. Solvent interactions with proteins as revealed by X-ray crystallographic studies. In Protein-solvent interactions (ed. R. B. Gregory) New York, pp: 143-189.

[126] Seki Y, Tomizawa T, Khechinashvili N, Soda K, 2002 Contribution of solvent water to the solution X-ray scattering profile of proteins. Biophys. Chem; 95: 235-252.

[127] Svergun D I, Richard S, Koch M H J, Sayers Z, Kuprin S, Zaccai G, 1998 Protein hydration in solution: experimental observation by X-ray and neutron scattering. Proc. Natl Acad. Sci. USA 95: 2267-2272.

[128] Brunschweiler R, Wright P E, 1994 Water self-diffusion model for protein-water NMR cross relaxation. Chem. Phys.Lett. 229, 75-81.

[129] Halle B, 1998 Water in biological systems: the NMR picture. In Hydration processes in biology (ed. M.-C. Bellisent-Funel), pp: 233-249.

[130] Jacobson B, 1953. Hydration structure of sodium deoxyribonucleic acid and its physicochemical properties. Nature; 172: 666-667. 
[131] Finer E G, 1973. Interpretation of deuterium magnetic nuclear resonance spectroscopic studies of the hydration of macromolecules. J Chem Soc Faraday II. 69: 1590-1600.

[132] Cornell B A, Pope J M, Troup G M, 1974. A pulsed NMR study of $\mathrm{D}_{2} \mathrm{O}$ bound to 1,2dipalmitoyl phosphatidylcholine. Chem. Phys. Lipids; 13:183-201.

[133] Cameron I L, Kanal K M, Keener C R, Fullerton G D, 1997. A mechanistic view of the non-ideal osmotic and motional behavior of intracellular water. Cell Biol. Int; 21: 99-113.

[134] Fenichel I R, Horowitz S B, 1963. The transports of non-electrolytes in muscle as a diffusional process in cytoplasm. Acta Physiol. Stand; 60, Suppl. $221: 1$.

[135] Fenichel I R, Horowitz S B, 1965. Diffusional specifity in water. Ann. N.Y. Acad. Sci; 125: 290-297.

[136] Agre P, Nielsen S, Ottersen O P, 2004. Towards a molecular understanding of water homeostasis in the brain. Neuroscience; 129: 849-50.

[137] Ling G N, Cope F W, 1969. Potassium Ion: Is the bulk of intracellular $\mathrm{K}^{+}$Adsorbed Science; 163: 1335-1336.

[138] Denisov V P, Halle B. 1996 Protein hydration dynamics in aqueous solution. Faraday Discuss; 103: 227-244.

[139] Nilsson L, Halle B. 2005. Molecular origin of time-dependent fluorescence shifts in proteins. Proc. Natl. Acad. Sci. USA, 102: 13867-13872.

[140] Hua L, Huang X, Zhou R, Berne B J, 2006. Dynamics of water confined in the interdomain region of a multidomain protein J. Phys. Chem B; 110: 3704-3711.

[141] Pal S, Zhao L, Zewail A H, 2003. Water at DNA surfaces: Ultrafast dynamics in minor groove recognition. Proc. Natl. Acad. Sci. USA; 100: 8113-8118. 
[142] Kumbhar M, Goel T, Mukherjee T, Pal H, 2004. Role of Micellar Size and Hydration on Solvation Dynamics : A Temperature Dependent Study in Triton-X-100 and Brij-35 Micelles. J Phys. Chem. B; 108(4): 19246-19254.

[143] Faeder J, Ladanyi B M, 2005. Solvation dynamics in reverse micelles: The role of headgroups-solute interactions $J$ Phys. Chem $B$; 109: 6732-6740.

[144] Abel S, Sterpone F, Badyopadhyay S, Marchi M, 2004. Molecular modeling and simulations of AOT-water reverse micelles in iso-octane: Structural and dynamical properties. J. Phys. Chem B; 108: 19458-19466.

[145] Wassall S R, 1996 Pulsed field-gradient-spin echo NMR studies of water diffusion in a phospholipid model membrane. Biophys. J; 71: 2724-32.

[146] Ling G N, Negendank, 1970. The physical state of water in frog muscles. Physiol. Chem. Phys; 2: 15 .

[147] Makarov V A, Andrews B K, Smith P E, Pettitt B M, 2000. Residence times of water molecules in the hydration sites of myoglobin. Biophys. J; 79: 2966-2974.

[148] Chen C N, Hoult D I, 1989 Biomedical magnetic resonance technology. Bristol and New York: Adam, Hilger; pp: 117-176.

[149] Hoult D I, Lauterbur P C, 1979 The sensitivity of the zeugmatographic experiment involving human samples. J Magn Reson; 34: 425-433.

[150] Cardenas-Blanco A, Tejos C, Irarrazaval P, Cameron I, 2008. Noise in magnitude magnetic resonance images. Concepts in MR, part A, 32: 409-416.

[151] Rice S O, 1944. Mathematical analysis of random noise. Bell System Technological Journal; 23: 282- 332 . 
[152] Abramowitz M, Stegun I A, 1970 Handbook of mathematical functions. Dover Publications, New York.

[153] Henkelman R M, 1986. Measurement of signal intensities in the presence of noise in MR images. Med Phys, 2: 232-233. Erratum in 1986; 13:544.

[154] Gudbjartsson H, Patz S, 1995. The Rician distribution of noisy MRI data. Magn Reson Med, 34:910- 914. Erratum in Magn Reson Med 1996; 36:332-333.

[155] Papoulis A, 1991. Probability, Random Variables and Stochastic Processes, 3. McGrawHill Inc; New York.

[156] Gradsthteyn I S, Ryzhik I M, 1965. Table of integrals, series and products. Academic Press, New York and London, $4^{\text {th }} \mathrm{ed}$.

[157] McGibney G, Smith M R, 1973. An unbiased signal-to-noise ratio measure for magnetic resonance images. Med Phys; 20:1077-107812.

[158] Miller A J, Joseph P M, 1993. The use of power images to perform quantitative analysis on low SNR MR images. Magn Reson Imag; 11:1051-1056.

[159] Koay C G, Basser P J, 2006 Analytically exact correction scheme for signal extraction from noisy magnitude MR signals. J Magn Reson; 179: 317-322.

[160] Sijbers J, den Dekker A J, Scheunders P, Van Dyck D, 1998. Maximum-likelihood estimation of Rician distribution parameters. IEEE Trans Med Imaging; 17:357-361.

[161] Close R A, Whiting J S, 1996. Maximum likelihood technique for blind noise estimation. In: Proceedings of SPIE Medical Imaging, Newport Beach, USA, pp: 18-28.

[162] Nezamzadeh M, Cameron I G, 2006. A new Rician noise bias correction. In: Proceedings of the 14th Annual Meeting of ISMRM, Seattle; pp: 346. 
[163] Nezamzadeh M, 2006. Rician Noise Corrected Multi-component Analysis of the MR Diffusion Signal Decay of Human Brain in Vivo, PhD thesis, Carleton Univ. Phys Dept. [164] Andersen A H, 1996 On the Rician distribution of noisy MRI data. Magn Reson Med; 36: 331-333.

[165] MacKay A L, Whittall K P, Adler J, Li D K, Paty D W, Graeb D A, 1994. In vivo visualization of myelin water in brain by magnetic resonance. Magn. Reson. Med; 31: 673677.

[166] Rutt B K, Mitchell J D, 1993. Myelin-selective imaging with 3D fast spin echo MRI, in Proc., SMRM, 12th Annual Meeting, New York, pp:137.

[167] Graham S J, Stanchev P L, Bronskill M J, 1996. Criteria for analysis of multicomponent tissue $\mathrm{T}_{2}$ relaxation data. Magn Reson Med; 35: 370-378.

[168] Marquart D W, 1963. An algorithm for least squares estimation of non-linear parameters. J Soc Industr Appl Math; 11: 431-441.

[170] Lawson C L, Hanson R J, 1974. Solving least squares problems. Englewood Cliffs, NJ Prentice-Hall.

[171] Pell G S, Briellmann R S, Waites A B, Abbott D F, Lewis D P, Jackson G D. Optimized clinical T2 relaxometry with a standard CPMG sequence. 2006 J Magn Reson Imaging; 23: $248-252$.

[165] Gambarota G, Cairns B E, Berde C B, Mulkern R V, 2001. Osmotic effects on T2 relaxation decay of in vivo muscle. Magn Reson Med; 46:592-599.

[166] Trantham E C, Rorschach H E, Clegg J S, Hazlewood C F, Nicklow RM, Wakabayashi N, 1984. Diffusive properties of water in Artemia cysts as determined from quasi-elastic neutron scattering spectra Biophys. J; 45: 927-38. 
[167] Middendorf H D, 1984. Biophysical applications of quasielastic and inelastic neutron scattering. Annu Rev Biophys Bioeng; 13: 425-451.

[168] Burton P, Drayer, 1988. Imaging of the Aging Brain. Radiology; 166: 785-796.

[169] Menon R S, Allen P S, 1991. Application of continuous relaxation time distributions to the fitting of data from model systems and excised tissue. Magn Reson Med; 20: 214-227. [170] Does M D, Snyder R E, 1995. T2 relaxation of peripheral nerve measured in vivo. Magn Reson Imaging;13: 575-580.

[171] Harrison R, Bronskill MJ, Henkelman RM, 1995. Magnetization transfer and T2 relaxation components in tissue. Magn. Reson. Med; 33: 490-496.

[172] Does M D, Snyder R E, 1996. Multi-exponential T2 relaxation in degenerating peripheral nerve. Magn. Reson. Med; 35: 207-213.

[173] Beaulieu C, Fenrich F R, Allen P S. Multicomponent water proton transverse relaxation and T2-discriminated water diffusion in myelinated and nonmyelinated nerve. Magn Reson Imaging; 16:1201-1210.

[174] Jolesz F A, Polak J F, Adams D F, Ruenzel P W, 1987. Myelinated and nonmyelinatednerves: comparison of proton MR properties. Radiology; 164: 89-91.

[175] Stewart W A, MacKay A L, Whittall K P, Moore G R, Paty D W, 1993 Spin-spin relaxation in experimental allergic encephalomyelitis. Analysis of CPMG data using a nonlinear least squares method and linear inverse theory. Magn Reson Med;29(6):767 -75.

[176] Stanisz G J, Henkelman R M, 1998. Diffusional anisotropy of T2 components in bovine optic nerve. Magn Reson Med; 40: 405-410. 
[177] Whittall K P, MacKay A L, Graeb D A, Nugent R A, Li D KB, Paty D W, 1997. In vivo measurement of T2 distributions and water contents in normal human brain. Magn.Reson. Med; 37: 34-43.

[178] Helms G, 2001. Volume correction for edema in single-volume proton MR spectroscopy of contrast-enhancing multiple sclerosis lesions. Magn Reson Med; 46(2):256-63.

[179] Laule C, Leung E, Li D KB, Traboulsee T, Oger J, Paty D W, 2003. Myelin water imaging in multiple sclerosis: quantitative correlations with histopathology. Mult Scler; Suppl $1:$ S35.

[180] Laule C, Vavasour I M, Moore G R W, Oger J, Li D K B, Paty D W, 2004. Water content and myelin water fraction in multiple sclerosis: a T2 relaxation study. $J$ Neurol ;251(3):284-93.

[181] Vasilescu V, Katona E, Simplaceanu V, Demco D, 1978 Water compartments in the myelinated nerve. III. Pulsed NMR results. Experientia; 34: 1443-1444.

[182] Brante, G, 1949 Studies on lipids in the nervous system. Acta Physiol. Scand. Suppl. 18, 63:1.

[183] Halle B, Denisov V P, 2001. Magnetic relaxation dispersion studies of biomolecular solutions. Meth. Enzymol; 338: 178-201.

[184] Fischer H W, Rinck P A, Van Haverbeke Y, Muller R N, 1990. Nuclear relaxation of human brain gray and white matter: analysis of field dependence and implications for MRI. Magn Reson Med; 16(2); 317-34.

[185] Whittall K P, MacKay A L, 1989. Quantitative interpretation of NMR relaxation data. $J$. Magn. Reson; 84: 134-152. 
[186] Belton P S, Jackson R R, Packer K J, 1972. Pulsed NMR studies of water in striated muscle. I. Transverse nuclear spin relaxation times and freezing effects. Biochim.Bbiophys. Acta; 286: 16-25.

[187] Hazlewood C F, Chang D C, Nichols B L, Woessner D E, 1974b. Nuclear magnetic resonance transverse relaxation times of water protons in skeletal muscle. Biophys. J. 14: 583606.

[188] Fung B M, 1977a. Proton and deuteron relaxation of muscle water over wide ranges of resonance frequencies. Biophys. J; 18: 235-239.

[189] Pissis P, Anagnostopoulou-Konsta A, Apekis L, 1987. A dielectric study of the state of water in plant stems $J$. Exp.Bot; 38: 1528-40.

[190] Talwar G P, Srivastava L M, 2004. Textbook of Biochemistry and Human Biology, $3^{\text {rd }}$ Ed. Prentice-Hall of India.

[191] Fitter J, Lechner R E, Dencher N A, 1999. Interactions of hydration water and biological membranes studied by neutron scattering J. Phys. Chem. B; 103: 8036-50.

[192] Miyoshi D, Sugimoto N, 2008. Molecular crowding effects on structure and stability of DNA. Biochim; 90: 1040-1051.

[193] Schoeniger J S, Aiken N, Hsu E, Blackband S J, 1994. Relaxation-Time and Diffusion NMR Microscopy of Single Neurons. J Magn Reson B; 103: 261-273.

[194] Hsu E, Aiken N R, Blackband S J, 1997. A study of diffusion isotropy in single neurons by using NMR microscopy. Magn Reson Med; 37: 624-627.

[195] Beaulieu C, Allen P S, 1994. Water diffusion in the giant axon of the squid: Implications for diffusion-weighted MRI of the nervous system. Magn. Reson. Med; 32:579-583.

[196] Marsh D, 1990. Handbook of Lipid Bilayers. CRC Press, Boca Raton, Florida. 
[197] LeBihan D, Turner R, Douek P, 1993. Is water diffusion restricted in human brain white matter? An echo-planar NMR imaging study Neuro. Rep. 4; 887-90.

[198] Moonen C T W, Pekar J, De Vleeschouwer M H M, Van Gelderen P, Van Zijl P C M and Des Pres D, 1991. Restricted and anisotropic displacement of water in healthy cat brain and in stroke studied by NMR diffusion imaging Magn. Reson. Med; 19; 327-32.

[199] Finkelstein A, 1987. Water movement through lipid bilayers, pores and plasma membranes: Theory and Reality. Wiley Interscience, New York, pp: 228.

[200] Troshin, A S, 1966 Problems of Cell Permeability. Revised and supplemented ed. $3^{\text {rd }}$ by Hell, M. G Translation ed. by Middas, W. F. Oxford, Pergamon Press.

[201] Grant S.C, Buckley D L, Gibbs S, Webb A G, Blackband S J, 2001. MR Microscopy of Multicomponent Diffusion in Single Neurons. Magn Reson in Med; 46:1107-1112.

[202] Callaghan P T, LeGros M A, Pinder D N, 1983. The measurement of diffusion using deuterium pulsed field gradient nuclear magnetic resonance. J. Chem. Phys; 79: 6372-6381.

[203] Volke F, Eisenblatter S, Galle J, Klose G, 1994. Dynamic properties of water at phosphatidylcholine lipid bilayer surfaces as seen by deuterium and pulsed field gradient proton NMR. Chem. Phys. Lipids; 70: 121-131.

[204] Rudakova M, Filippov A, Skirda V, 2004 Water diffusivity in model biological membranes. Appl Magn Reson; 27: 519-526.

[205] Barkovich A J, Kjos B O, Jackson D E Jr, Norman D, 1988. Normal maturation of the neonatal and infant brain: MR imaging at 1.5 T. Radiology;166: 173-180.

[206] Pandya D N, Seltzer B, 1986. The topography of commissural fibers. Two Hemispheres, One Brain. Functions of the Corpus Callosum. Ed Lepore F, Ptito M, New York, pp: 47-73. 